\title{
Detailanalysen von Transferprozessen
}

In diesem Kapitel werden die Detailanalysen der Bearbeitungsprozesse der Lernenden dargestellt. Es werden ausgewählte Bearbeitungen von Lernendenpaaren in Transfersituationen analysiert und miteinander in Beziehung gesetzt. In Hinsicht auf das Forschungsinteresse dieser Arbeit folgen die deskriptiven Analysen vor allem den folgenden Zielen:

Deskriptive Beschreibung von Transferprozessen: Es wird der Frage nachgegangen, wie sich Transferprozesse in alltäglichen Unterrichtssituationen gestalten. $\mathrm{Zu}$ diesem $\mathrm{Zweck}$ werden die Bearbeitungsprozesse der Lernendenpaare in Transfersituationen rekonstruiert, mit dem Ziel Transferprozesse zu identifizieren und detailliert zu beschreiben.

Rekonstruktion der Erklärungsmodelle der Lernenden: Ein wesentliches Augenmerk der Analysen der Bearbeitungsprozesse ist die Rekonstruktion der Erklärungsmodelle und Vorstellungen der Lernenden. Die Frage dabei ist zum einen, welche Erklärungsmodelle und Vorstellungen die Lernenden bei der Bearbeitung der Transferaufgaben aktivieren und zum anderen, inwieweit diese Transferprozesse unterstützen oder womöglich behindern.

Auf dieser Grundlage werden mit dem Ziel der Charakterisierung von Transferprozessen mehrere Vergleiche in Rahmen von komparativen Analysen vorgenommen. Hierzu werden einerseits die individuellen Vorstellungen und Erklärungsmodelle der Partnerinnen und Partner miteinander verglichen, um individuelle Unterschiede und Gemeinsamkeiten herauszustellen, die einen Einfluss auf Lösungsprozesse und respektive die Transferprozesse haben. Auf einer weiteren Ebene des Vergleichs werden die Bearbeitungsprozesse verschiedener Paare zu der gleichen Transferaufgabe vorgenommen, um die Spezifika der Bearbeitungs- und Transferprozesse in einem größeren Zusammenhang miteinander Beziehung zu setzen. Diese Schritte 
werden für verschiedene Transferaufgaben im gleichen inhaltlichen Kontext wiederholt. Hierbei ist das Ziel zu vergleichen, inwieweit sich Transferprozesse in unterschiedlichen Transfersituationen mit demselben Gegenstand des Transfers ähneln oder unterscheiden.

Zuletzt sollen Transferprozesse im Rahmen einer längerfristigen Unterrichtseinheit untersucht werden. Vor diesem Hintergrund erfolgen die beschriebenen Analysen anhand der Daten von drei verschiedenen Datenerhebungen zu unterschiedlichen Zeitpunkten der Unterrichtseinheit. Diese bilden die übergeordnete Ebene des Vergleichs der Analyseergebnisse. In Abschnitt 5.1 werden die Analysen der Partnerarbeiten zur Einführung von Brüchen als Teile eines Ganzen geschildert. Die Analysen in Abschnitt 5.2 umfassen Partnerarbeiten, in denen die Schülerinnen und Schüler Transferaufgaben zu Brüchen als Teile beliebiger Größen bearbeiten und im dritten Abschnitt5.3 ist der inhaltliche Kern der Analysen das Verfahren zum Kürzen von Brüchen als Vergröbern einer Einteilung im Zusammenhang mit der Gleichwertigkeit von Brüchen. Abschließend werden die Ergebnisse der Analysen der drei Datenerhebungen in Abschnitt 5.4 zusammengeführt.

\subsection{Anteile von einem Ganzen}

In diesem Abschnitt werden die Analysen der Daten aus der ersten Datenerhebung dargestellt. Dazu werden zunächst in einem Überblick die verwendeten Lernmaterialien beschrieben. Diese umfassen ein interaktives animiertes Lösungsbeispiel, zwei unvollständige Lösungsbeispiele sowie ausgewählte Transferaufgaben. Die Beschreibung der Materialien erfolgt auf sachanalytischer Ebene mit Bezug auf die enthaltenen Grundvorstellungen. Diese normativen Analysen stellen den Ausgangspunkt für die Beschreibung der für eine Lösung der Transferaufgaben erforderlichen Transferprozesse.

Lösungsbeispiel: Zur Einleitung der Arbeitsphase lesen die Lernenden ein zweiteiliges animiertes Lösungsbeispiel am Computer. Die beiden Teile des Lösungsbeispiels sind in ihren Endzuständen auch in den Arbeitsheften der Lernenden abgebildet und werden jeweils durch fokussierende Fragestellungen begleitet.

Teil a) des Lösungsbeispiels veranschaulicht die Herstellung des Bruchs $\frac{5}{8}$ an einer Kreisrepräsentation (Endzustand siehe Abbildung 5.1). In den Animationen des Lösungsbeispiels wird zunächst ein Kreis in acht gleich große Teile zerlegt. Ein Teil davon wird farblich als $\frac{1}{8}$ markiert bevor im nächsten Schritt nacheinander fünf Teile eingefärbt werden, sodass insgesamt $\frac{5}{8}$ des ganzen Kreises markiert sind. 


\section{Mathematik} heute

\section{a b i schroedel}

a) Erklăre, wie $\frac{5}{8}$ eines Ganzen entsteht. Stelle das Ganze mit Kreisflächen dar.

Lösung:

Die Kreisfläche (die Pizza) wird in 8 gleich große Teile zerlegt.

Ein Stück davon ist $\frac{1}{8}$.

Davon werden dann 5 Teile genommen. Das sind dann $\frac{5}{8}$.

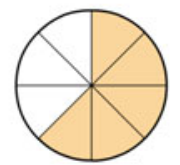

Diesen Ablauf kann man auch mit Pfeilen darstellen:

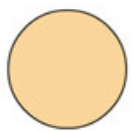

1

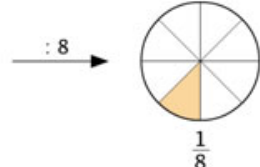

$\frac{1}{8}$

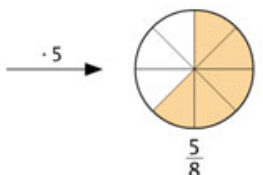

口内 M II

\section{Mathematik} heute

b) Erkläre, wie $\frac{3}{8}$ eines Ganzen entsteht. Stelle das Ganze mit Rechtecken dar.

\section{Lösung:}

Das Rechteck (der Kuchen) wird in 8 gleich große Teile zerlegt.

Ein Stück davon ist $\frac{1}{8}$.

Davon werden dann 3 Teile genommen. Das sind dann $\frac{3}{8}$.

Diesen Ablauf kann man auch mit Pfeilen darstellen:

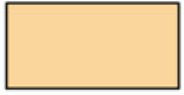

1

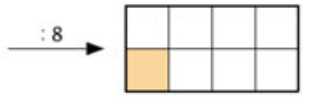

$\frac{1}{8}$

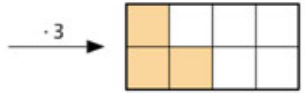

$\frac{3}{8}$

Abbildung 5.1 Endzustand des animierten Lösungsbeispiels 1a (oben) und 1b (unten) 
Im Anschluss wird dieses Bruchherstellungsverfahren in einem ikonischen und symbolischen Pfeildiagramm dargestellt und die jeweiligen Herstellungshandlungen mit den entsprechenden Rechenoperatoren :8 zum Teilen des ganzen in acht gleich große Teile und $\cdot 5$ zum Vervielfachen bzw. 'Nehmen' von fünf Teilen verknüpft.

Teil b) des Lösungsbeispiels vollzieht analog die Anteilbildung des Bruchs $\frac{3}{8}$ in verkürzter Form anhand einer Rechteckdarstellung nach (Endzustand siehe Abbildung 5.1).

In den Arbeitsheften der Lernenden werden die statisch abgebildeten Lösungsbeispiele jeweils mit einer fokussierenden Fragestellung begleitet:

Lösungsbeispiel Teil a): Warum erhält man $\frac{5}{8}$ und nicht $\frac{8}{5}$ ? Begründe.

Lösungsbeispiel Teil b): Beschreibe mit eigenen Worten, wie man aus einem Ganzen $\frac{3}{8}$ erhält.

Die fokussierenden Fragen beziehen sich auf das Bruchherstellungsverfahren als Kernkonzept des Lösungsbeispiels und bestehen in der Aufforderung zur Richtigstellung einer häufig auftretenden Fehlvorstellung sowie in einer Aufforderung zur Erklärung des Verfahrens im Lösungsbeispiel mit eigenen Worten. Die erste fokussierende Fragestellung soll so die Lernenden dazu anleiten über die unterschiedlichen Bedeutungen von Zähler und Nenner zu reflektieren und herauszustellen, dass die Zahl im Nenner die Anzahl der gleich großen Teile angibt, in die das Ganze eingeteilt ist, und die Zahl im Zähler die Anzahl der markierten Teile repräsentiert. Im Gegensatz dazu wird in der zweiten fokussierenden Fragestellung dazu aufgefordert, das im Lösungsbeispiel dargebotene Herstellungsverfahren für den Bruch $\frac{3}{8}$ in eigenen Worte wiederzugeben. Das didaktische Ziel ist hierbei die Loslösung vom Lernmaterial anzuregen und auf die selbstständige Anwendung des Verfahrens zur Beschreibung der Herstellung anderer Brüche vorzubereiten.

Ausgewählte Transferaufgaben: Im Anschluss an die interaktiven animierten Lösungsbeispiele folgt eine Serie von Übungs- und Transferaufgaben (siehe Tabelle 5.1). Detaillierte Aufgabenbeschreibungen und Erläuterungen der Transferprozesse erfolgen an den entsprechenden Stellen in diesem Kapitel. 
Tabelle 5.1 Aufgabensequenz - Anteile von einem Ganzen

\begin{tabular}{l|l|l}
\hline & Aufgabe & Zentrale Transferprozesse \\
\hline $1 \& 2$ & $\begin{array}{l}\text { Unvollständige } \\
\text { Lösungsbeispiele }\end{array}$ & $\begin{array}{l}\text { Übertragung des Bruchherstellungsverfahrens auf } \\
\text { neue Brüche und ikonische Darstellungen }\end{array}$ \\
\hline 4 & $\begin{array}{l}\text { Bruchdarstellung } \\
\text { an einer Strecke }\end{array}$ & $\begin{array}{l}\text { Übertragung des Verfahrens zur Bruchdarstellung } \\
\text { von einer Flächendarstellung in eine lineare } \\
\text { Darstellung }\end{array}$ \\
\hline
\end{tabular}

\subsubsection{Unvollständige Beispiele}

Das erste unvollständige Beispiel ist strukturgleich mit den zuvor studierten Lösungsbeispielen und dient als Unterstützung zur Übertragung der visualisierten Handlung in den Lösungsbeispielen in eine eigenständige Anwendung. Die zentralen Schritte der Bruchherstellung sind entsprechend der Lösungsschritte im Lösungsbeispiel strukturiert und mit Handlungsanweisungen in seitlichen Sprechblasen kommentiert.

Im ersten Schritt sollen zunächst die Rechenoperationen zur Herstellung von einem Achtel aus einem Ganzen und die notwendige Rechenoperation zum Vervielfachen zu $\frac{6}{8}$ in einem Pfeilschema mit ikonischen Repräsentationen ergänzt werden. Direkt im Anschluss sollen die gleichen Operationen in einem symbolischen Pfeilschema eingetragen werden. Im Vordergrund steht hier die Analogie zwischen der Bruchherstellung auf ikonischer und symbolischer Repräsentationsebene. Die ikonische Repräsentation zur Veranschaulichung ist analog zum Lösungsspiel ein Kreis. Der einzige Unterschied ist an dieser Stelle, dass im Lösungsbeispiel der Bruch $\frac{5}{8}$ und in diesem ersten unvollständigen Beispiel der Bruch $\frac{6}{8}$ hergestellt wird. Somit kann die Einteilung in Achtel durch eine Division mit 8 aus dem Lösungsspiel übernommen werden, im Anschluss muss jedoch mit 6 und nicht mit 5 multipliziert werden.

Im strukturgleichen Aufgabenteil b) des ersten unvollständigen Beispiels sollen die Rechenoperationen zur Herstellung des Bruchs $\frac{4}{5}$ notiert werden. In Analogie zum zweiten Lösungsbeispiel wird die Bruchherstellung in dieser Teilaufgabe anhand einer Rechteckrepräsentation veranschaulicht. Im Gegensatz zu Aufgabenteil a) soll zusätzlich der nach der ersten Rechenoperation entstehende Stammbruch $\frac{1}{5}$ eingetragen werden (siehe Abb. 5.2).

In Aufgabe 2 sollen die Lernenden die Herstellung der Brüche $\frac{2}{3}$ (Teil a) und $\frac{3}{8}$ (Teil b) beschreiben und die Brüche in einer Kreisrepräsentation (a) bzw. einer Rechteckrepräsentation (b) darstellen. Im Gegensatz zu Aufgabe 1 ist die Bearbeitung nicht vorstrukturiert und mit Handlungsanweisungen versehen, sondern es 
b) Erkläre, wie $\frac{4}{5}$ eines Ganzen entsteht. Stelle das Ganze durch ein Rechteck dar.

\section{Lösung:}

Das Rechteck (z.B. ein Blatt Papier) wird in 5 gleich große Teile zerlegt, davon werden dann 4 genommen.
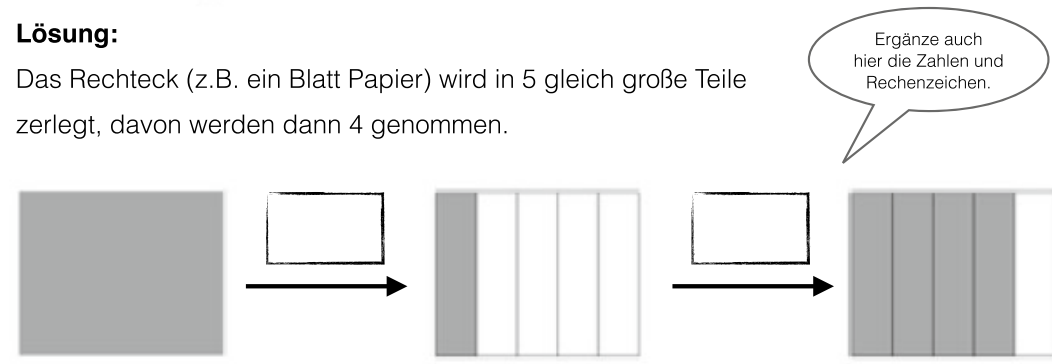

Als Pfeilbild mit Zahlen geschrieben:
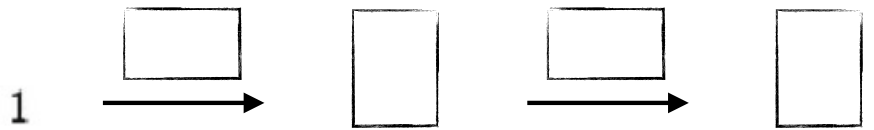

Abbildung 5.2 Teilaufgabe b) des ersten unvollständigen Lösungsbeispiels (Aufgabe 1b)

sind lediglich ein kariertes Feld für die Lösung und eine leere Repräsentation zum Einzeichnen des jeweiligen Bruchs vorgegeben. In der Aufgabenstellung ist zudem die Hilfestellung enthalten, dass auch ein Pfeildiagramm gezeichnet werden kann (siehe Abb. 5.3).

Transferprozesse in Aufgaben 1 und 2: Für die Bearbeitung der unvollständigen Beispiele in den Aufgaben 1 und 2 sind vor allem drei Transferprozesse von zentraler Bedeutung:

1. Die Übertragung des Bruchherstellungsverfahrens von den Lösungsbeispielen in eine eigene Durchführung,

2. die Übertragung des Bruchherstellungsverfahrens auf neue Brüche und ikonische Figuren sowie

3. die Übersetzung der Herstellungshandlung zwischen der ikonischen und symbolischen Repräsentationsebene.

Im Hinblick auf die Ausbildung von Grundvorstellungen zu Bruchzahlen steht in dieser Aufgabe die Grundvorstellung vom Bruch als Operator (vgl. Kapitel3) im 
a) Das Ganze ist hier durch einen Kreis dargestellt.

1) Erkläre, wie $\frac{2}{3}$ eines Ganzen entsteht. Du kannst auch ein Pfeilbild zeichnen.

2) Zeichne den Bruchteil ein.

Lösung:

1)

2)
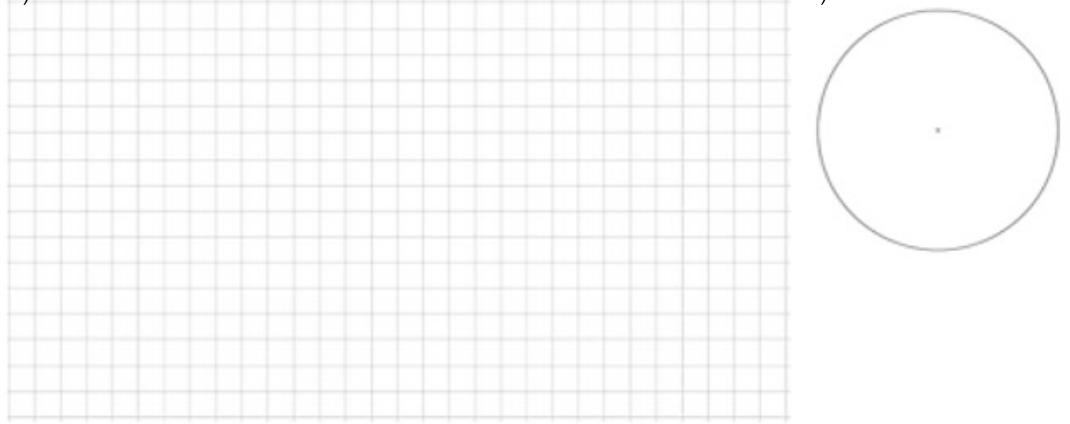

Abbildung 5.3 Teilaufgabe a) des zweiten unvollständigen Lösungsbeispiels (Aufgabe 2a)

Vordergrund. Mit der Ausbildung der Operatorvorstellung sind folgende Annahmen verbunden, die in die Konzeption des Unterrichtsmaterials einbezogen wurden:

- Die Einübung des Bruchherstellungsverfahrens : $n \cdot m$ als mentales Muster führt zum Aufbau einer Grundvorstellung.

- Dieses mentale Muster kann durch eine Generalisierung unabhängig von Zahlen und Repräsentationen angewendet werden.

- Die Darstellung als Pfeilschema in ikonischer und symbolischer Form dient als Hilfe zum Anknüpfen an das Vorwissen der Lernenden.

\section{Bennet \& Julius - Verknüpfung der Herstellungshandlung mit symbolischen Rechenoperationen}

Die Schüler Bennet und Julius bilden ein sehr kommunikationsfreudiges Schülerpaar. Ihre Ergebnisse im Vortest deuten auf sehr unterschiedliche Vorwissensstände hin. Während Bennet im Vortest bereits $85 \%$ Aufgaben fehlerfrei lösen konnte, erreichte sein Partner Julius im Vortest lediglich ein Testergebnis von 35\%. Damit sind ihre Testleistungen in den höchsten und niedrigsten Ergebnisquartilen einzuordnen. 


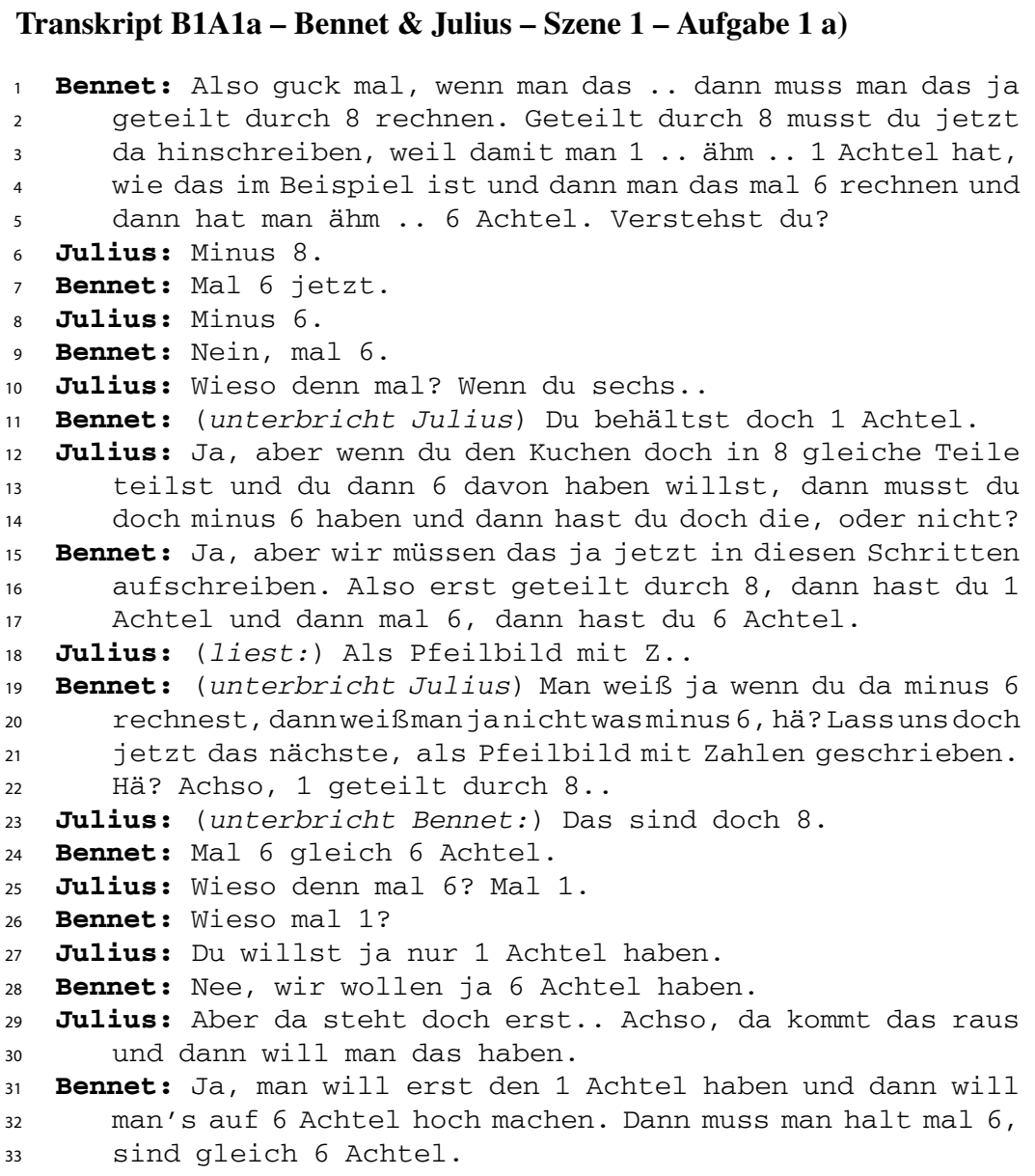

Unmittelbar nach dem Lesen der Aufgabenstellung formuliert Bennet seinen Lösungsweg laut denkend (1-5). Er erklärt, dass man das Ganze zunächst ,geteilt durch 8 rechnen“ (2) müsse, „damit man 1 .. ähm 1 Achtel hat“ (3). AnschlieBend müsse man „das mal 6 rechnen und dann hat man ähm .. 6 Achtel“ (4-5). Er formuliert die Bruchherstellung anhand der Folge von durchzuführenden Rechenoperationen auf symbolischer Ebene und benennt dabei auch die Ergebnisse der Rechenoperationen: Durch Teilen durch 8 erhält man den Bruch $\frac{1}{8}$. Multipliziert man diesen mit 6 erhält man den Bruch $\frac{6}{8}$. Seinen Lösungsweg begründet Bennet 
damit, dass das Vorgehen so sei, „wie das im Beispiel ist“ (4), wodurch deutlich wird, dass er sich auf das Herstellungsverfahren im Lösungsbeispiel bezieht. Die kurzen Pausen in seiner Erklärung deuten darauf hin, dass er die Rechenoperatoren Schritt für Schritt aus dem Lösungsbeispiel überträgt und somit eine Analogie zu dem Lösungsbeispiel herstellt. Dabei bezieht er sich ausschließlich auf die symbolische Rechenhandlung und geht nicht auf die ikonische Repräsentation der Bruchherstellung ein. Seine Formulierung ,,weil damit man 1 .. ähm .. 1 Achtel hat“ (3) lässt zudem annehmen, dass er nicht einfach die entsprechenden Rechenoperationen überträgt, sondern diese für sich auch begründet und plausibilisiert. Seine Rückfrage ,verstehst du“ (5) stützt diese Annahme. Er fragt seinen Partner nicht, ob seine Lösung richtig ist oder ob sein Partner ihm zustimmt, sondern er fragt, ob sein Partner das genauso verstanden hat.

Sein Partner Julius widerspricht Bennets Lösungsweg umgehend und korrigiert, dass man „minus 8“ (6) bzw. „minus 6“ (8) rechnen müsse. Er bezieht sich damit auf eine Handlung mit konkreten Gegenständen - einem Kuchen - und erklärt: „wenn du den Kuchen doch in 8 gleiche Teile teilst und du dann 6 davon haben willst, dann musst du doch minus 6" (12-14) rechnen. In diesen Äußerungen wird deutlich, dass er den zweiten Teiloperator zur Bruchherstellung als ein gegenständliches Wegnehmen deutet. Es ist nicht hinreichend zu klären, ob er sich bei seinem Einwurf ,minus 8“ (6) lediglich versprochen hat und eigentlich „,minus 6“ (8) meint oder ob er hier annimmt, dass man vom Ganzen 8 subtrahieren müsse, um auf $\frac{1}{8}$ zu kommen. Deutlich wird jedoch, dass er die Operatoren nicht als ein Teilen und Vervielfachen deutet, sondern die wörtliche Formulierung aus dem Lösungsbeispiel „davon werden dann 5 Teile genommen. Das sind dann $\frac{5}{8}$ “ überträgt. Anders als Bennet stellt Julius einen Gegenstandsbezug zum Aufteilen eines Kuchens her, der zunächst in acht gleich große Teile geteilt wird, von denen man schließlich 6 Teile wegnehmen möchte. Das Wegnehmen entspricht in seiner Vorstellung nicht einer Multiplikation mit 6, sondern einer Subtraktion von 6 Teilen. Entsprechend kann er Bennets Lösungsweg nicht nachvollziehen: „wieso denn mal?“ (10).

Bennet reagiert auf den Einwurf seines Partners mit einem Verweis auf das Vorgehen im Lösungsbeispiel und erklärt, dass man ,das ja jetzt in diesen Schritten aufschreiben“ (15-16) solle, womit er sich auf die Teiloperatoren des Bruchherstellungsverfahrens bezieht: ,Also erst geteilt durch 8, dann hast du 1 Achtel und dann mal 6, dann hast du 6 Achtel“" (16-17). Während Julius noch einmal im Lösungsbeispiel liest, versucht Bennet den Einwand von Julius nachzuvollziehen. Er ergänzt, dass man gar nicht wisse, wovon man 6 abziehen soll (19-20) und setzt an, die Rechenoperationen im symbolischen Pfeilschema zu ergänzen „Achso, 1 geteilt durch 8..“ (22). 
Julius hat in der Zwischenzeit noch einmal im Lösungsbeispiel nachgelesen und merkt an, dass man ,,mal 1“ (25) rechnen müsse, denn man will zunächst ,,ja nur 1 Achtel haben“ (27). Er nimmt folglich Bennets Argumentation auf, dass man zunächst teilen und dann multiplizieren muss. Diese Schrittfolge überträgt er im Sinne einer Übergeneralisierung auf die Stammbruchherstellung, indem er erklärt, dass man um $\frac{1}{8}$ zu erhalten zunächst durch 8 teilen und dann mit 1 multiplizieren müsse, wobei er möglicherweise nicht erkennt, dass die Division durch 8 bereits zu $\frac{1}{8}$ führt und die Multiplikation mit 1 nicht notwendig ist. Da man im Zwischenschritt ,ja nur 1 Achtel haben“ (27) möchte, müsse man mit 1 multiplizieren, falls man das Schema übertragen will.

Auf den Widerspruch von Bennet, der den Gedankengang seines Partners scheinbar nicht nachvollziehen kann, wendet sich Julius noch einmal dem Lösungsbeispiel zu und liest erneut den Rechenweg (29-30). Hier erkennt er, dass bereits die Division mit 8 zu dem Bruch $\frac{1}{8}$ führt.

Abschließend erläutert Bennet noch einmal den Rechenweg: ,Ja man will erst den 1 Achtel haben und dann will man's auf 6 Achtel hoch machen. Muss muss man halt mal 6, sind gleich 6 Achtel“" (31-33). Bemerkenswert in dieser abschließenden Erläuterung ist Bennets Formulierung ,,man will erst den 1 Achtel haben und dann will man's auf 6 Achtel hoch machen", in der deutlich die multiplikative Denkweise von Bennet zu erkennen ist. Mit seiner Formulierung "hochmachen“ bezieht er sich auf die Abbildung von 1 auf 6, die er nicht als wiederholte Addition eines Teils deutet, sondern als Vervielfachen des Teils im Sinne einer Streckung. Diese Vorstellung steht in starkem Kontrast zu der subtraktiven Vorstellung seines Partners Julius, der zuvor den zweiten Teiloperator als Wegnehmen von Teilen interpretiert hat.

\section{Transkript B1A1b - Bennet \& Julius - Szene 2 - Aufgabe 1 b)}

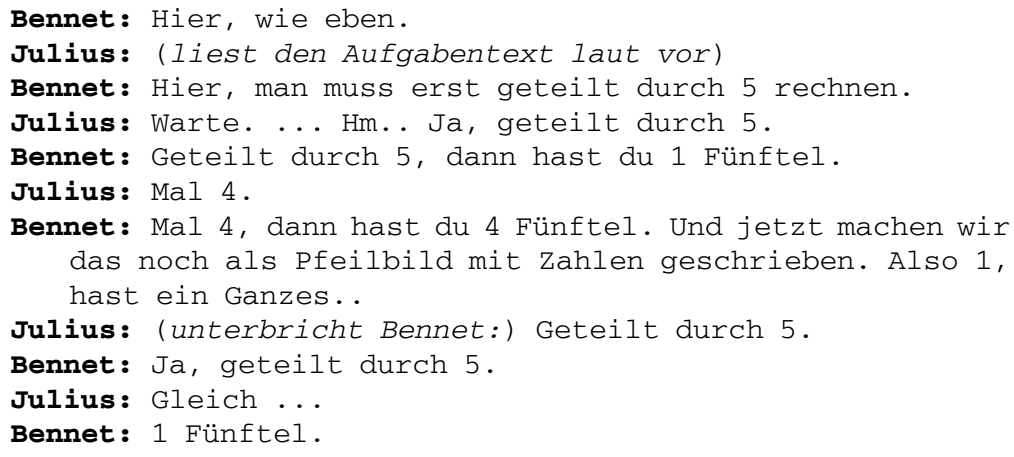




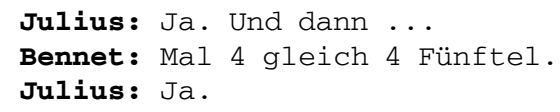

Bereits in der anschließenden Bearbeitung von Teilaufgabe b), in der die Herstellung des Bruchs $\frac{4}{5}$ erklärt werden soll, ist zu erkennen, dass Bennet das Verfahren zur Bruchherstellung bereits zu verallgemeinern beginnt und spontan die entsprechenden Rechenoperationen nennt. Noch während Julius mit dem Lesen der Aufgabenstellung beschäftig ist sagt Bennet: „Hier muss man erst geteilt durch 5 rechnen“ (36), ,dann hast du 1 Fünftel“ (38). Es gelingt ihm scheinbar mühelos das Verfahren auf die Herstellung anderer Brüche zu übertragen.

Sein Partner Julius kann der Geschwindigkeit, mit der sein Partner die Rechenoperationen ergänzt, zunächst nicht folgen. Er bestätigt dann jedoch ,,ja, geteilt durch 5“ (37) die erste Rechenoperation und ergänzt dann selber ,mal 4“ (39), was Bennet bestätigt.

In der Notation der Rechenoperatoren und Zwischenergebnisse in dem vorstrukturierten symbolischen Pfeilschema wirkt Julius weiterhin unsicher, bestätigt jedoch jeweils die Vorschläge von Bennet. Es ist anzunehmen, dass Julius an dieser Stelle mehr Zeit zum Nachdenken benötigt als sein Partner, der sehr schnell agiert.

\section{Transkript B1A2a/b - Bennet \& Julius - Szene 3 - Aufgabe 2 a) und b)}

Bennet: Lass uns ein Pfeilbild machen. Komm, wie eben. Erst so ein Kästchen.. nein.

Julius: (blättert zurück:) Gucken wir doch einfach ab hier.

Bennet: Ja, nein, aber diesmal müssen wir es mit einem Kreis machen.

Julius: Wieso? Es geht auch ein Rechteck.

Bennet: Wir machen einfach 1, das ist dann ein Ganzes ... mal 2, also 1 mal 2 gleich ... Nein, geteilt durch hä?

Julius: Warte.

Bennet: Geteilt durch 3 müssen wir erst machen.

Julius: Nein, das ist doch falsch.

Bennet: Doch, dann ist es 1 Drittel. Denn wenn man geteilt durch 3 macht ist Eins 1 Drittel..

Julius: (unterbricht Bennet:) Nein, da musst du doch erst mal 1, dann ist es 1 Drittel.

Bennet: Hier guck (blättert zurück) da auf dem anderen Blatt ... Hier ist doch das Ganze und wir müssen das nicht geteilt durch 8, sondern geteilt durch 3 rechnen.

Julius: Ah, ja.

Bennet: Geteilt durch 2 ist gleich..

Julius: (unterbricht Bennet:) Durch 3 . 


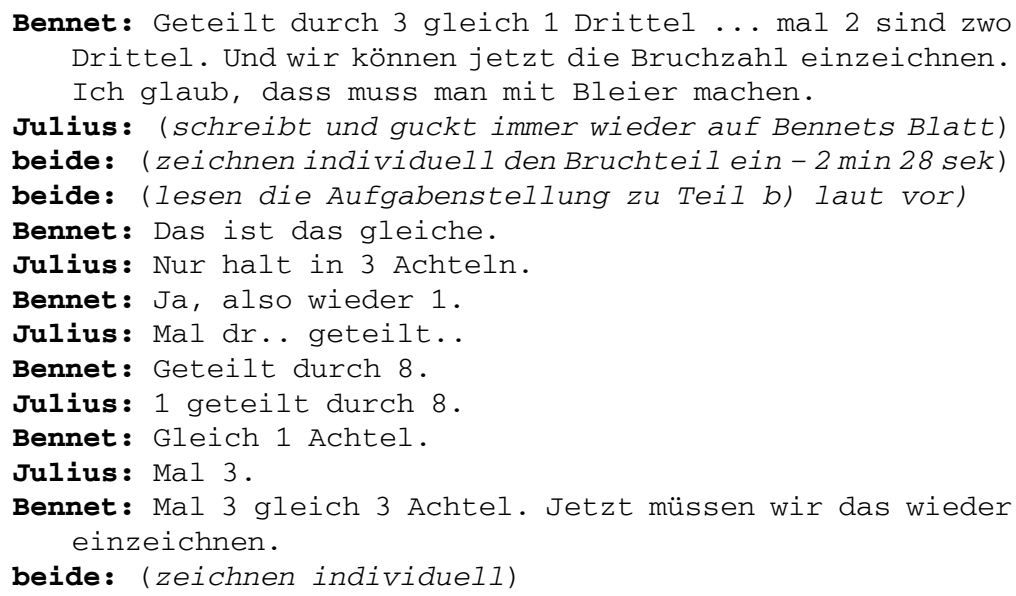

In dem zweiten unvollständigen Beispiel (Aufgabe 2) sollen die Lernenden zunächst anhand einer Kreisrepräsentation erklären, wie $\frac{2}{3}$ hergestellt werden. Im zweiten Aufgabenteil soll anhand einer Rechteckrepräsentation erklärt werden, wie der Bruch $\frac{3}{8}$ hergestellt wird.

Unmittelbar nach dem Lesen der Aufgabenstellung schlägt Bennet vor ein Pfeilbild ,wie eben“ (1) zu zeichnen, womit er sich auf das Pfeilschema im Lösungsbeispiel und im ersten unvollständigen Beispiel bezieht. Dazu möchte er ,erst so ein[en] Kasten“ zeichnen. Der Vorschlag einen Kasten zu zeichnen, kann einerseits so interpretiert werden, dass er den Bruchteil in einem Rechteck darstellen möchte und aus diesem Grund zunächst einen Kasten als Repräsentation eines Ganzen zeichnen müsste. Es ist auch möglich, dass er einen Kasten zeichnen möchte, in den er wie im ersten unvollständigen Beispiel die Zahl 1 oder eine Rechenoperation eintragen möchte. Seine spätere Äußerung ,,nein, aber diesmal müssen wir es mit einem Kreis machen“ (4-5) stützt wiederum die erste Annahme, dass er ein Pfeilschema mit ikonischen Darstellungen anfertigen möchte, aber übersehen hat, dass die Aufgabenstellung die Darstellung in einem Kreis vorgibt. Es kann angenommen werden, dass er sich für den Lösungsweg an den Teilschritten des Lösungsbeispiels und des ersten unvollständigen Beispiels orientiert.

Die Feststellung, dass die Aufgabenstellung eine Bruchdarstellung im Kreis vorsieht, veranlasst ihn dazu, das Verfahren mit dem Ziel den Aufwand zu verringern abzuändern: ,Wir machen einfach 1, das ist dann ein Ganzes ... mal 2, also 1 mal 2 gleich ... Nein, geteilt durch hä?“ (7-8), ,geteilt durch 3 müssen wir erst machen“ (10), „dann ist es ein Drittel“ (12). Bennet überspringt demnach die iko- 


\section{Lösung:}

1)

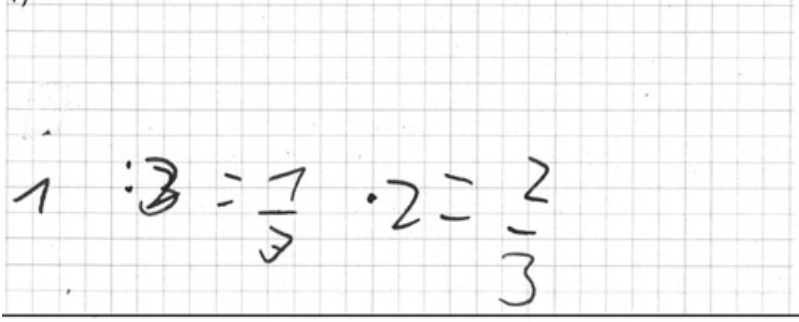

2)

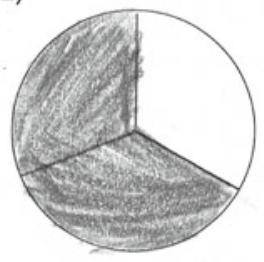

2)

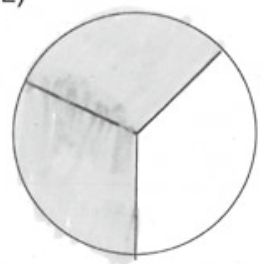

1)

$7: 3=\frac{1}{3} \cdot 2=\frac{2}{3}$

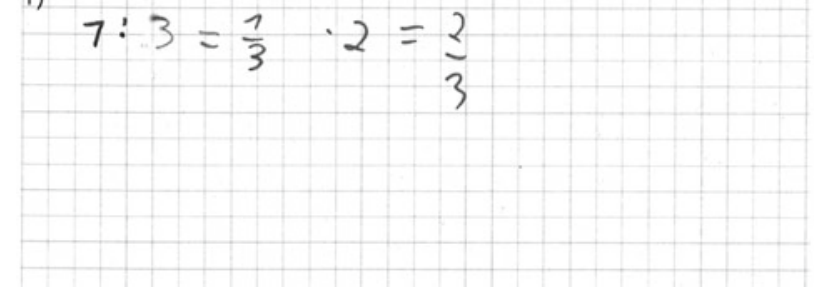

1)

Abbildung 5.4 Bennets (oben) Julius (unten) Lösung von Aufgabe 2 a)

nische Darstellung der einzelnen Herstellungsschritte und geht sofort zur Notation der Herstellungsschritte in symbolischer Form über.

Dabei beginnt er anders als im Lösungsbeispiel und im ersten unvollständigen Beispiel nicht mit dem Nenneroperator zum Teilen des Ganzen, sondern mit dem Zähleroperator zum Vervielfachen des Ganzen. Er erkennt vermutlich nicht, dass ein Vertauschen der Reihenfolge keine Auswirkungen auf das Ergebnis hat, und korrigiert seinen Rechenweg entsprechend des bisherigen Vorgehens im Lern- und Aufgabenmaterial. Ungeachtet der Reihenfolge der Anwendung der Operatoren ist zu erkennen, dass er den Zähleroperator mit einer Multiplikation und den Nenneroperator mit einer Division verbindet, was die Annahme bestärkt, dass er mit den Positionen der Zahlen in einer Bruchdarstellung eine feste Rechenoperation assoziiert: Die Zahl im Zähler vervielfacht, die Zahl im Nenner teilt. Auch ohne die einzelnen Herstellungsschritte zeichnerisch darzustellen hinterfragt und begründet er seine Rechnung $1: 3=\frac{1}{3}$ (siehe Abb. 5.4). Er sagt: „Denn wenn man geteilt durch 3 macht ist Eins 1 Drittel“" (12-13). Hierbei ist nicht zu erkennen, ob er sich diesen Schritt bildlich vorstellt oder die Rechnung auf symbolischer Ebene hinterfragt. Er 
schließt seine Erklärung der Bruchherstellung ohne erkennbare Schwierigkeiten: „Geteilt durch 3 gleich 1 Drittel ... mal 2 sind zwo Drittel“ (22-23).

Während Bennet nahezu mühelos das Verfahren zur Bruchherstellung übertragen, anwenden und, durch Auslassung der Darstellung in einem ikonischen Pfeilschema, anpassen kann, zeigt sein Partner Julius deutliche Unsicherheiten. $\mathrm{Zu}$ Beginn der Bearbeitung blättert Julius zurück zum statischen Lösungsbeispiel im Arbeitsheft und schlägt vor, dort abzugucken (3). Dieses Zurückblättern bringt zum Ausdruck, dass er die einzelnen Herstellungsschritte zu diesem Zeitpunkt noch nicht in dem Maße verinnerlicht hat, dass er die Schrittfolge ohne Vorlage anwenden kann. Zudem ist zu erkennen, dass er die Übergeneralisierung aus der Bearbeitung des ersten unvollständigen Beispiels, dass man zur Herstellung eines Stammbruchs $\frac{1}{n}$ erst durch $n$ teilt und dann mit 1 multipliziert, noch nicht überwunden hat, sodass diese erneut zum Tragen kommt. Dies wird an der Stelle deutlich, an der er die Rechnung von Bennet unterbricht und einwirft „Nein, da musst du doch erst mal 1, dann ist es 1 Drittel“ (14-15). Zur Aufklärung geht Bennet auf ihre Bearbeitung des ersten unvollständigen Beispiels zurück und erklärt ihm daran, dass sie dort auch allein durch ein Teilen durch 8, den Bruch $\frac{1}{8}$ erhalten haben. Zudem nimmt er einen analogen Vergleich vor, indem er erklärt: „Hier ist doch das Ganze und wir müssen das nicht geteilt durch 8, sondern geteilt durch 3 rechnen“ (16-18). Diese Verbindung erkennt Julius an und stimmt im Weiteren dem Vorgehen Bennets im Allgemeinen zu. Er korrigiert Bennets Versprecher und stellt richtig, dass sie im ersten Schritt nicht ,geteilt durch 2“ (20), sondern „,durch 3“ (21) rechnen müssen.

Bei der Notation des Rechenwegs orientiert sich Julius nicht an der Struktur des Lösungsbeispiels und des ersten unvollständigen Beispiels, sondern übernimmt die Notation von seinem Partner. Insgesamt ist zu erkennen, dass Julius noch große Unsicherheiten bei der Anwendung des Verfahrens zur Bruchherstellung hat und die einzelnen Herstellungsschritte zwar nachvollziehen kann, jedoch nicht eigenständig ohne Vorlage anzuwenden vermag. Diese Interpretation wird dadurch gestützt, dass er in der Bearbeitung der zweiten Teilaufgabe zur Erläuterung der Herstellung des Bruchs $\frac{3}{8}$ mit seinem Partner im Wechsel die notwendigen Rechenoperationen nennt und auch eigenständig notiert. Hierbei orientiert er sich bei der Notation ebenfalls nicht an der Darstellung im Lösungsbeispiel und im ersten unvollständigen Beispiel, sondern an der von Bennet übernommenen Notation aus Aufgabenteil a) (vgl. Abb. 5.5).

Die mathematisch inkorrekte Notation des Rechenweges geht auf Bennet zurück, der scheinbar versucht den Schreib- und Zeichenaufwand zu minimieren. Zunächst verzichtet Bennet auf die Darstellung der Herstellung in einem ikonischen Pfeilschema und auch für die symbolische Notation verzichtet er auf die Darstellung als Pfeilschema. Stattdessen schreibt er den Rechenweg in einer Art Gleichungskette 
Abbildung 5.5 Julius

Notation des Rechenweges zur Herstellung von $\frac{3}{8}$

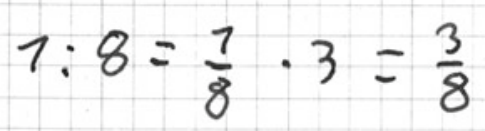

auf, wobei er nicht beachtet, dass an den jeweiligen Enden der Gleichungskette nicht das gleiche steht. Dennoch sind die einzelnen Rechenschritte bzw. Teiloperatoren abzulesen.

Beide Schüler haben keine Schwierigkeiten bei der zeichnerischen Darstellung der Bruchteile in den vorgegebenen Figuren. Sie teilen die Figur in drei bzw. acht gleiche Teile und färben die entsprechende Anzahl von Teilen. Sie stellen die Zeichnungen in stiller Individualarbeit her, was annehmen lässt, dass sie das Verfahren zur Bruchherstellung auf ikonischer Ebene durchaus anwenden können.

Insgesamt lassen sich die Interpretationen der Bearbeitungen von Bennet und Julius anhand der folgenden Deutungshypothesen zusammenfassen:

- Bennet und Julius übertragen sehr unterschiedliche Aspekte aus dem Lösungsbeispiel auf die unvollständigen Beispiele: Während Bennet die Struktur des Bruchherstellungsverfahrens als Hintereinanderausführung zweier Teiloperatoren überträgt, anwendet und an andere Brüche anpasst, überträgt sein Partner Julius eher kontextbezogene Aspekte der Bruchherstellung. Julius überträgt insbesondere die Formulierung, dass ein Ganzes in $n$ gleiche Teile geteilt wird, von denen dann $m$ Teile weggenommen werden. Dieses Wegnehmen übersetzt er mit einer Subtraktion als Rechenoperation für den Zähleroperator. Seine Erklärungen sind deutlich an die Handlung mit konkreten Gegenständen gebunden, während sein Partner Bennet bereits vornehmlich auf symbolischer Ebene mit Zahlen und Rechenhandlungen argumentiert und operiert.

- Obgleich Bennet die schrittweise Herstellungshandlung aus dem Lösungsbeispiel auf die Bearbeitung des zweiten unvollständigen Beispiels überträgt, wandelt er die Notationsweise ab. Er zeichnet keine Pfeilschemata, sondern schreibt eine Rechnung auf, in der beide Teiloperatoren nacheinander aufgeführt werden. Julius übernimmt die Notation seines Partners und bedarf einer Vorlage zur Ausführung der einzelnen Herstellungsschritte.

- Die ikonische Darstellung von Bruchteilen gelingt beiden Schülern ohne einen direkten Bezug zum Bruchherstellungsverfahren herzustellen. Das Vorgehen dabei wird an keiner Stelle diskutiert und die Zeichnungen in individuellen Arbeitsphasen unabhängig voneinander angefertigt. Dabei unterteilen die bei- 
den Schüler die Figuren zunächst in die dem Nenner entsprechende Anzahl von Teilen und Färben schließlich die im Zähler angegebene Anzahl.

\section{Can, Philip \& Glen - Getrennte Betrachtung von ikonischer und symbolischer Darstellung}

Da Glens eigentliche Partnerin Johanna in der ersten Doppelstunde der Datenerhebung fehlt, arbeitet er zusammen mit Philip und Can. Im Vortest erreichte Can 50\%, Philip 55\% und Glen $65 \%$ der möglichen Punkte, sodass alle drei Testergebnisse den mittleren Niveaustufen zugeordnet werden konnten. Das Arbeitsverhalten der drei Schüler kann anhand des vorhergehenden Stundenverlaufs als unkonzentriert beschrieben werden. Sie albern viel herum und widmen sich nur in geringem Maße den vorliegenden Lern- und Aufgabenmaterialien. Da ihr unruhiges Verhalten die nahesitzenden Paare zunehmend ablenkt, wurden sie zuvor bereits wiederholt von der Lehrkraft ermahnt. Das Lösungsbeispiel zum Einstieg in die Arbeitsphase haben sie nicht eingehend gelesen, sondern lediglich überflogen, indem sie die einzelnen Segmente des animierten Lösungsbeispiels ,,durchgeklickt“ haben, sobald der Segmentaufbau abgeschlossen war und die Möglichkeit bestand zum nächsten Schritt überzugehen. Es findet keine Kommunikation über das Lösungsbeispiel statt und auch die fokussierenden Fragen haben sie zu diesem Zeitpunkt nicht beachtet.

\section{Transkript B1A1a - Can, Philip \& Glen - Szene 1 - Aufgabe 1 a)}

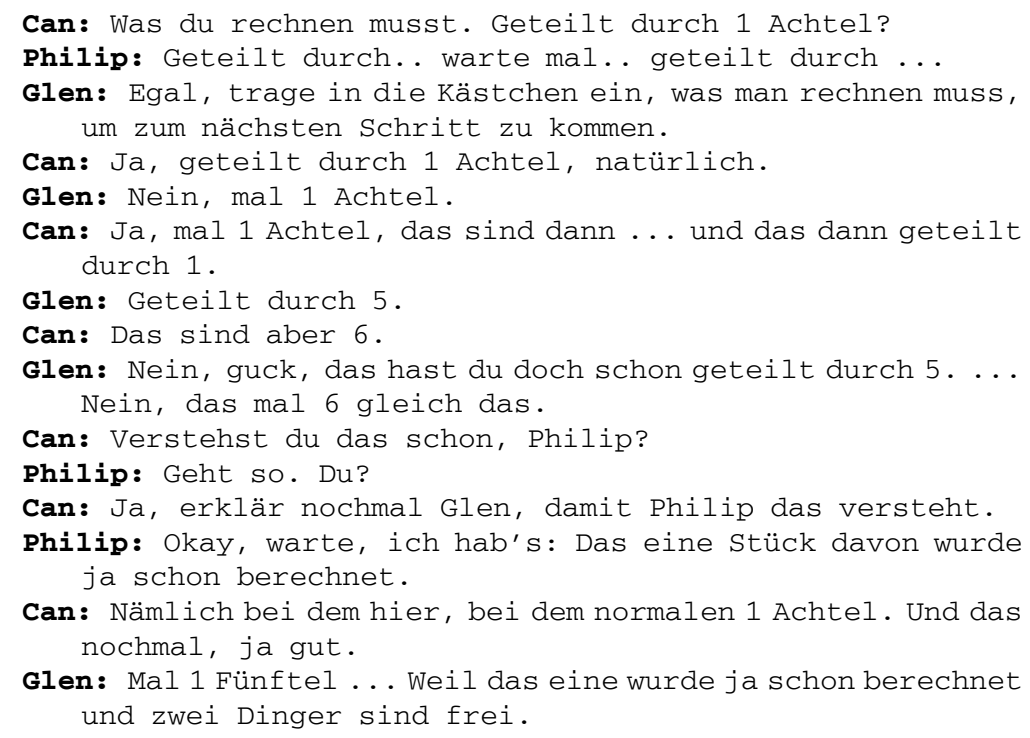




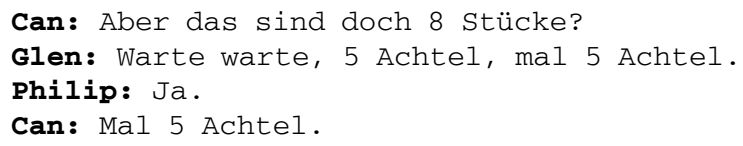

Bereits zu Beginn wird deutlich, dass die drei Schüler das Lösungsbeispiel nicht eingehend genug gelesen haben und für die Bearbeitung der unvollständigen Beispiele nicht auf die Informationen aus dem Lernmaterial zurückgreifen. Stattdessen versuchen sie anhand der im Aufgabenmaterial abgebildeten ikonischen Pfeildarstellung die gesuchten Rechenoperationen herzuleiten und ihren Rechenweg zu plausibilisieren (Abb. 5.6).

\section{Lösung:}

Die Kreisfläche (z.B. ein Kuchen) wird in 8 gleich große Teile zerlegt, davon werden dann 6 genommen.
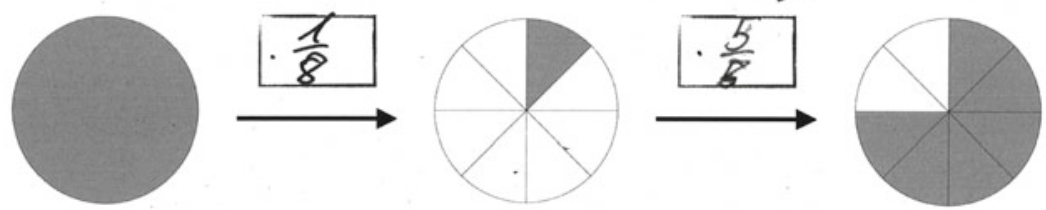

Als Pfeilbild mit Zahlen geschrieben:
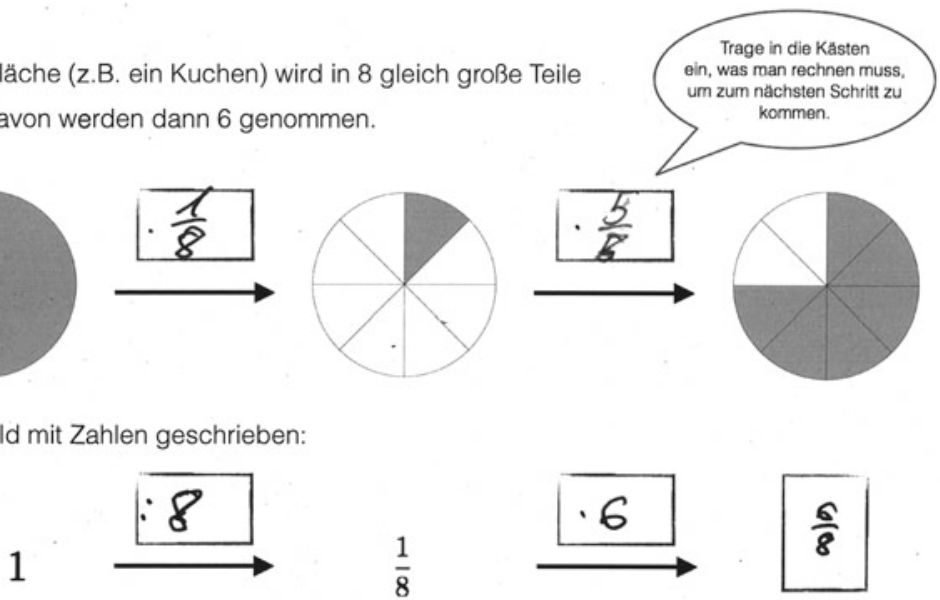

Abbildung 5.6 Glens Lösung von Aufgabe 1 a)

Zunächst versuchen sie die Rechenoperationen im ikonischen Pfeilschema zu ergänzen. Ihre Vorschläge haben einen ratenden Charakter. Es werden Rechenoperationen genannt, die nicht begründet werden. Die Vorschläge für den ersten Rechenschritt sind „geteilt durch 1 Achtel“ $(1,5)$ und „mal 1 Achtel“ (6). Es ist anzunehmen, dass Can mit seinem Vorschlag ,geteilt durch 1 Achtel“ $(1,5) \mathrm{zu}$ rechnen auf eine Verkleinerung des Ganzen abzielt. Da er keine näheren Erläuterungen zu seinem Vorschlag gibt, können verschiedene gedankliche Hintergründe vermutet werden. Es kann angenommen werden, dass er mit der Division die Operationsvorstellung einer Verkleinerung verbindet. Da $\frac{1}{8}$ weniger als ein Ganzes ist, 
schlägt er demnach vor ,geteilt“ zu rechnen. Ferner steht zur Frage, warum er durch $\frac{1}{8}$ teilen möchte und nicht durch 8 . Hier kann angenommen werden, dass er einen Bruch notieren will, da es in der Unterrichtsreihe um Brüche geht. Er verbindet mit dieser Operation jedoch ein Teilen durch 8. Diese Interpretation wird durch seine weitere Ausführung auf die Erwiderung von Glen gestärkt. Er nimmt Glens Erwiderung auf, dass sie nicht durch ein Achtel teilen müssen, sondern mit einem Achtel multiplizieren müssen und fügt an: ,Ja, mal 1 Achtel, das sind dann ... und das geteilt durch 1“ (7-8). Diese Ergänzung kann so interpretiert werden, dass er ähnlich wie Julius Übergeneralisierung in der zuvor geschilderten Bearbeitung zur Herstellung von einem Achtel zunächst im Nenneroperator durch 8 teilt und dann mit dem Zähleroperator 1 multiplizieren möchte.

Sein Partner Glen stellt Cans Vorschlag durch ein Achtel zu teilen entgegen, dass sie „mal 1 Achtel rechnen müssen“ (6). Er begründet diesen Vorschlag nicht, es ist jedoch anzunehmen, dass er hierbei nicht die Multiplikation mit einem Bruch meint. Dies wird insbesondere durch seinen Vorschlag für die nachfolgende Rechenoperation deutlich, für die er vorschlägt ,geteilt durch 5“ (9) zu rechnen, um von einem Achtel zu sechs Achteln zu kommen. Er wirkt verwirrt bezüglich der Wirkungen der Rechenoperationen und es ist anzunehmen, dass er dem Lösungsbeispiel entnommen hat, dass in einem Schritt dividiert und in einem Schritt multipliziert wird. Entsprechend wählt er jeweils eine der Rechenoperationen für einen Schritt. Als Can anmerkt, dass es am Ende „aber 6“ (10) Achtel sein sollen, denkt er noch einmal über seine Vorschläge nach und stellt fest, dass die Rechenoperation ,durch 5“ (11) nicht passt. Er korrigiert seinen Vorschlag und erkennt, dass ein Achtel vervielfacht werden muss, so dass er sagt: „Nein, das [1 Achtel] mal 6 gleich das [6 Achtel]“ (12). In der Folge überlegt er noch einmal neu und erklärt schließlich „,mal 1 Fünftel ... Weil das eine wurde ja schon berechnet und zwei Dinger sind frei“ (20-21). An dieser Stelle wird deutlich, dass er mit der Multiplikation ,mal 1 Fünftel“ die Addition von fünf Teilen verbindet. Da im Zwischenschritt bereits einer von acht Teilen markiert ist, müssen für die Darstellung von $\frac{6}{8}$ noch fünf Teile zusätzlich markiert werden, sodass insgesamt sechs Teile gefärbt und zwei Teile bzw. „Zwei Dinger“ nicht gefärbt bzw. „,frei“ sind. Auch hier ist anzunehmen, dass er ähnlich wie Can einen Bruch in die Kästchen für die Rechenoperationen eintragen möchte, da es im Unterricht um Brüche geht, aber eigentlich an eine Division und Multiplikation mit einer natürlichen Zahl denkt.

Zuletzt einigen sich die Schüler auf die zweite Rechenoperation „,mal 5 Achtel“ (23), mit der sie eine Addition von fünf Achteln verbinden. Ihr Rechenweg kann so zusammengefasst werden, dass sie zunächst durch acht teilen wollen, um ein Achtel zu erhalten. Für diese Rechenoperation notieren sie jedoch $\cdot \frac{1}{8}$. Zu diesem 
einen Achtel wollen sie in der Folge fünf Achtel hinzufügen bzw. addieren. Sie notieren jedoch die Rechenoperation $\frac{5}{8}$.

In ihren Herleitungen des Rechenwegs ist zu erkennen, dass sie im Prinzip ein Teilen und ein Vervielfachen als Herstellungsschritte erkannt haben, jedoch diese nicht notieren, sondern entsprechend des Unterrichtsinhalts „Brüche“ Bruchoperatoren notieren.

Philip hält sich aus der Interaktion weitgehend heraus und gibt zu verstehen, dass er das alles nicht so richtig versteht (14). Er kann dann aber nachvollziehen, dass wenn schon ein Achtel vorhanden ist noch fünf Achtel zu sechs Achteln fehlen, weil ,das eine Stück davon wurde ja schon berechnet“ (16-17).

\section{Transkript B1A1a - Can, Philip \& Glen - Szene 2 - Aufgabe 1 a)}

In der Folge gehen die Schüler zum Ergänzen der Rechenoperationen im symbolischen Pfeilschema über. In ihrer Kommunikation wird deutlich, dass sie dabei keine Verbindung zwischen der ikonischen Darstellung der Herstellung und der rechnerischen Herstellungshandlung herstellen.

Nachdem sie mit Hilfe der Lehrkraft geklärt haben, was in diesem Aufgabenteil zu tun ist (26-30) erklärt Glen: ,guck, 1 geteilt durch 8 gleich 1 Achtel“ (31), was durch die Lehrkraft bestätigt wird. Die Lehrkraft weist ferner darauf hin, dass es „dasselbe wie hier [in der ikonischen Darstellung]“ (32-33) sei. Can merkt an, dass sie das doch bereits aufgeschrieben haben $(35,39)$. 
Glen führt im weiteren aus, dass sie im zweiten Schritt „1 Achtel mal 6 gleich 6 Achtel“ (40-41) rechnen und notieren müssen. Er widerspricht zudem Philips Einwand, dass sie „mal 5“ rechnen müssen, ,weil man hat ja schon 1 Achtel“ (42). Glen korrigiert ihn und erklärt „,mal 6, mal 5 ist 5 und wir brauchen 6 Achtel“ (43).

Während Can und Philip in ähnlicher Weise argumentieren, wie beim Ergänzen der Rechenoperatoren im ikonischen Pfeilschema, ändert Glen seine Argumentation beim Ergänzen des symbolischen Pfeilschemas. Er beschreibt die Teiloperatoren richtigerweise als :8 und 6 und widerlegt seine Rechnung, die er eine Zeile darüber notiert hat. Er hinterfragt diesen Unterschied jedoch nicht und seine Partner übernehmen seine Lösung.

Zusammenfassend kann festgestellt werden, dass die Schüler die ikonische und symbolische Darstellung der Bruchherstellung nicht miteinander in Beziehung setzen, sondern getrennt voneinander unterschiedliche Rechenoperationen derselben Funktion zuweisen. Während sie anhand des ikonischen Pfeilschemas versuchen den dargestellten Bildern eine Bedeutung zu zuweisen, scheint ihnen die symbolische Darstellung des Rechenweges keine Schwierigkeiten zu bereiten. Anstatt wie in der ikonischen Darstellung mit dem zweiten Teiloperator fünf Teile hinzufügen zu wollen, erklärt Glen, dass ein Achtel mit 6 multipliziert werden müsse, da man schließlich 6 Achtel und nicht 5 Achtel erhalten wolle. Während sie in der ikonischen Pfeildarstellung versucht haben Bruchoperatoren für die jeweiligen Schritte anzugeben, steht dies bei der Ergänzung des symbolischen Pfeilschemas nicht zur Diskussion und sie notieren Rechenoperatoren mit natürlichen Zahlen. Diese Beobachtungen stützen die Interpretation, dass die ikonische Darstellung der Bruchherstellung für sie in keiner Beziehung zum Rechenweg auf symbolischer Darstellungsebene steht.

\section{Transkript B1A1b - Can, Philip \& Glen - Szene 3 - Aufgabe 1 b)}

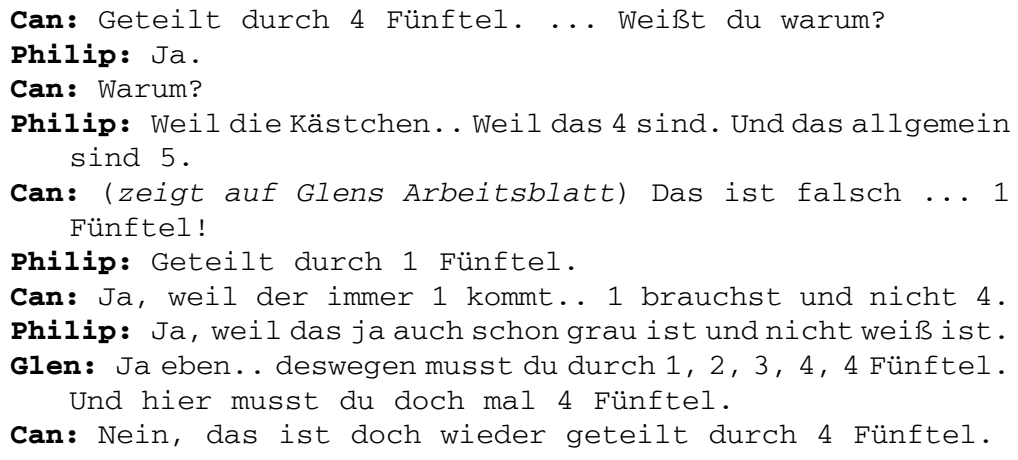


Glen: Nein, guck: 1 mal 4, das ist was anderes als 5 geteilt durch 4.

Philip: (zeigt auf Glens Arbeitsblatt) Das ist falsch, weil das eine ist da ja schon berechnet worden.

Can: Ja.

Glen: Und dann wollt ihr da jetzt 3 Fünftel hinschreiben oder was?

Can: Nein, 1 Viertel kommt da hin. Mal 1 Viertel.

Philip: Also das hier ist ja schon berechnet. einer muss sowieso also ...

Can: Mal 1 Viertel ... mal 1 Fünftel.

Glen: Mal 3 Fünftel. Das eine haben wir ja schon. Das mal das Fünftel, das Fünftel und das Fünftel. Und mal nimmt man 1 mal 4, also 4 Fünftel.

Can: Also mal 4 Fünftel?

Glen: Ja. (Nach Ca. 13 Minuten Ablenkung und erneutem flüchtigen Durchsehen des Lösungsbeispiels:) Als Pfeilbild mit Zahlen geschrieben ... geteilt durch 4 gleich 1 Fünftel mal 4 gleich 4 Fünftel.

Can: Ja.

\section{Lösung:}

Das Rechteck (z.B. ein Blatt Papier) wird in 5 gleich große Teile zerlegt, davon werden dann 4 genommen.
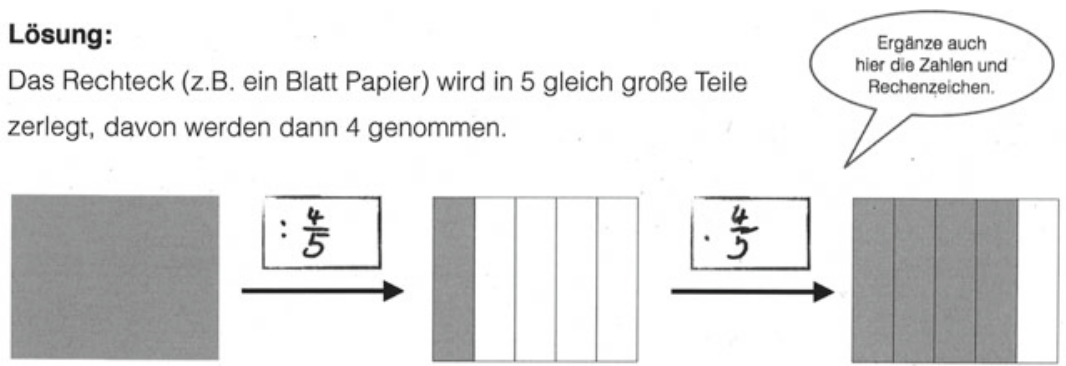

Als Pfeilbild mit Zahlen geschrieben:
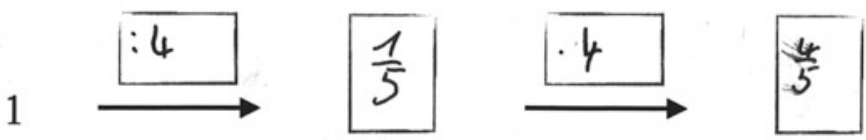

Abbildung 5.7 Glens Lösung von Aufgabe 1 b) 
Ein ähnliches Bild zeigt sich in ihrer Bearbeitung von Teilaufgabe b), in der sie im selben Format die Herstellung des Bruchs $\frac{4}{5}$ erklären sollen.

In Analogie zu ihrer Bearbeitung von Aufgabenteil a) nennt Can als erste Rechenoperation zu Herstellen eines Fünftels aus einem Ganzen ,geteilt durch 4 Fünftel“ (44). Da er sich jedoch scheinbar sehr unsicher ist, fragt er seine Partner, warum das so sei, worauf Philip mit Bezug auf die Teile des Ganzen in der ikonischen Darstellung antwortet: ,Weil das 4 sind. Und allgemein sind es 5“ (47-48). Er argumentiert somit im Sinne einer Subtraktion von $\frac{4}{5}$. Mit ,,allgemein sind es 5“ meint er vermutlich, dass das Ganze in fünf Teile geteilt ist, von denen ein Teil markiert ist. Demnach müsse man „4 Fünftel“ abziehen, wobei er mit wahrscheinlich meint, dass vier Teile abgezogen werden müssen. Das Abziehen der vier Teile verbindet er mit einer Division.

Mit Blick auf das Arbeitsblatt seines Partners Glen, der ebenfalls bereits : $\frac{4}{5}$ an der Stelle der ersten Rechenoperation notiert hat (Abb. 5.7), kommentiert Can im Gegensatz zu seinem anfänglichen Vorschlag, dass das falsch sei und man durch „1 Fünftel“ (49-50) teilen müsse. Er begründet dies damit, dass ,der immer 1 kommt.. 1 brauchst und nicht 4“ (52), womit er auf die 1 im Zähler des Zwischenergebnisses $\frac{1}{5}$ Bezug nimmt. Philip stimmt ihm zu und erklärt weiter, dass das so sei, da ,das ja auch schon grau ist und nicht weiß ist" (53). Demnach folgt Philip dem neuen Vorschlag von Can, dass $1: \frac{1}{5}=\frac{1}{5}$ sei. Sie nehmen keinen Bezug auf ihren ersten Vorschlag und die dazu gehörige Erklärung von Philip.

Glen widerspricht seinen Partnern und merkt an, dass, gerade weil man vier Teile wegnehmen wolle, man durch $\frac{4}{5}$ teilen müsse: „Ja, eben.. deswegen musst du durch 1, 2, 3, 4, 4 Fünftel“ (54). Er folgt demnach demselben Gedanken, den Philip zu Beginn geäußert hat in Anlehnung an ihre Lösung von Aufgabenteil a).

Für die zweite Rechenoperation fügt Glen an ,und hier musst du dich mal 4 Fünftel“ (55), womit er vermutlich meint, dass das Zwischenergebnis $\frac{1}{5}$ mit 4 multipliziert werden müsse. Anders als noch bei Aufgabenteil a), in der er den zweiten Rechenoperator im Sinne einer Addition beschrieben hat, beschreibt er nun eine Multiplikation.

Philip folgt ihrer Lösung von Aufgabenteil a) und argumentiert für eine Addition von drei Fünfteln, weil ,das hier [1 Fünftel] ist ja schon berechnet“ (65-66). Darauf hin gibt Glen ihm indirekt Recht, indem er erklärt ,,mal 3 Fünftel. Das eine haben wir ja schon. Das mal das Fünftel, das Fünftel und das Fünftel" (68-69), wobei angenommen werden kann, dass er ,plus“" meint, obgleich er „,mal“ sagt. Er fügt hinzu: „Und mal nimmt man 1 mal 4, also 4 Fünftel“ (69-70). Diese Rechenoperation notieren daraufhin auch Can und Philip.

Die Beiträge von Can sind über den gesamten Bearbeitungsverlauf nur schwer nachzuvollziehen. Zunächst schlägt er selber „durch 4 Fünftel“ (1) vor, was er 
wenig später als falsch bezeichnet und sagt, dass man durch ,1 Fünftel“ (49-50) teilen müsse. Wenig später argumentiert er erneut für seinen ersten Vorschlag bevor er zuletzt für den zweiten Rechenoperator „mal 1 Viertel“ (64) vorschlägt. Seine Äußerungen können so gedeutet werden, dass er ratend an der Interaktion zwischen den Partnern teilnimmt. Sein Vorschlag ,mal 1 Viertel“ kann aber auch so interpretiert werden, dass er sich damit auf eine Multiplikation mit 4 bezieht. Dies ist jedoch nicht schlüssig zu klären.

Im Anschluss an diese Sequenz folgt eine längere Ablenkungsphase, in der die Schüler nahezu 13 Minuten lang herumalbern, die umliegenden anderen Partnerarbeiten stören und nebenbei gelangweilt und zum Teil ohne hinzuschauen das animierte Lösungsbeispiel am Bildschirm durchlaufen lassen. Glen nimmt schließlich die Bearbeitung der Aufgabe wieder auf und notiert entsprechend seiner Eintragungen der Rechenoperatoren im ikonischen Pfeilschema :4 und ·4, was seine Partner übernehmen. Hierbei ist anzunehmen, dass er mit :4 eine Subtraktion von vier Teilen assoziiert, da er sagt ,geteilt durch 4 gleich 1 Fünftel“ (74). Unmittelbar darauf gehen die Schüler zur Bearbeitung des zweiten unvollständigen Beispiels über.

\section{Transkript B1A2 - Can, Philip \& Glen - Szene 4 - Aufgabe 2}

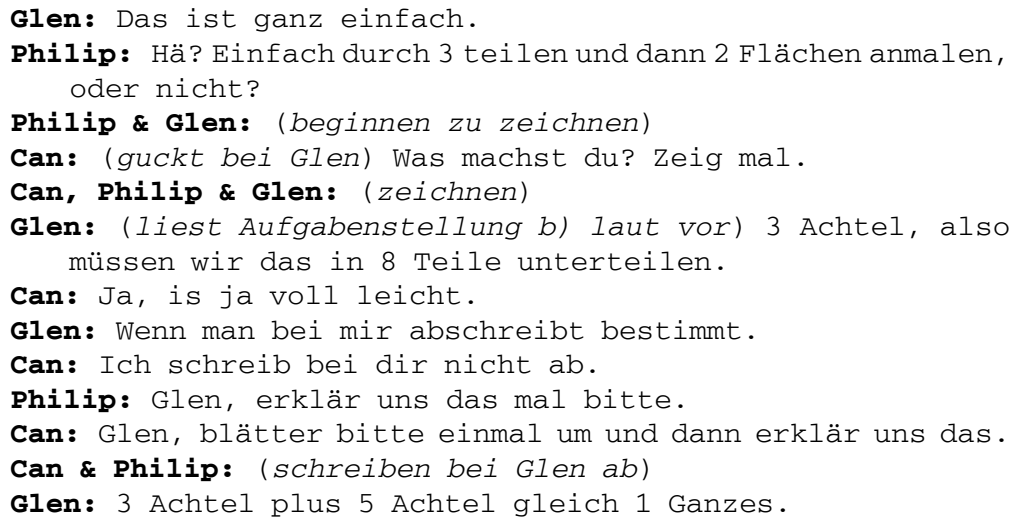

Auch in ihrer Bearbeitung des zweiten unvollständigen Beispiels ist eine Trennung von der ikonischen und symbolischen Darstellungsebene zu erkennen. Philip und Glen beginnen unmittelbar nach dem Lesen der Aufgabenstellung mit der zeichnerischen Darstellung des Bruchs $\frac{2}{3}$. Ungeachtet seiner Schwierigkeiten in der Bearbeitung des ersten unvollständigen Beispiels, weiß Philip sofort, was zu tun ist. Er erklärt: „Einfach durch 3 teilen und dann 2 Flächen anmalen, oder nicht?“ (2-3). 
Seine Beschreibung der Vorgehensweise zum Einzeichnen von $\frac{2}{3}$ in einer Kreisrepräsentation zeigt, dass er im Kontext der ikonischen Darstellung von Brüchen, den Zähler und Nenner eines Bruchs als Teiloperatoren interpretiert: „,durch 3 teilen und dann 2 Flächen anmalen“. Er kann dieses Herstellungsschema jedoch nicht auf die symbolische Darstellung übertragen, sodass er Hilfe bei seinem Partner Glen sucht und ihn um eine Erklärung bittet (12).

Glen bemerkt, dass er die Aufgabe „ganz einfach“ (1) findet und beginnt sofort mit der zeichnerischen Darstellung des Bruchs $\frac{2}{3}$. Für die Darstellung des Bruchs $\frac{3}{8}$, beschreibt er: ,3 Achtel, also müssen wir das in 8 Teile unterteilen“ (7-8). Ähnlich wie Philip hat er keine Schwierigkeiten, anhand der Zähler und Nenner eines vorgegebenen Bruchs abzuleiten, in wie viele Teile das Ganze geteilt werden muss und wie viele dieser Teile farbig markiert werden müssen. Anstelle einer Beschreibung der Bruchherstellung notiert Glen je eine Addition: $\frac{2}{3}+\frac{1}{3}=1$ Ganzes und $\frac{3}{8}+\frac{5}{3}=1$ Ganzes (vgl. Abb. 5.8). In seinen Äußerungen wird nicht ersichtlich, warum er diese Gleichungen aufschreibt. Obwohl sowohl im Lösungsbeispiel sowie im ersten unvollständigen Beispiel die die Bruchherstellung stets anhand eines Pfeilschemas erklärt wurde bzw. erklärt werden sollte, wird diese Struktur nicht übertragen. Die Schwierigkeiten der Schüler beim Ergänzen der Rechenoperationen im ersten unvollständigen Beispiel stützen jedoch die Annahme, dass sie das Pfeilschema nicht als Hilfsmittel zur Strukturierung und Darstellung der Bruchherstel-

1)

$$
\frac{2}{3}+\frac{1}{3}=1 \text { Ganzes }
$$

1)

$$
\frac{3}{8}+\frac{5}{8}=1 \text { Ganzes }
$$

2)

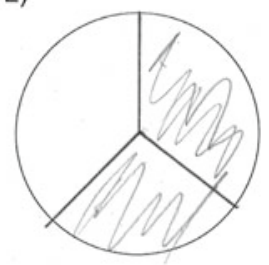

2)

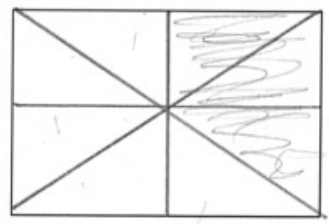

Abbildung 5.8 Glens Lösung von Aufgabe 2a (oben) und 2b (unten) 
lung betrachten, sondern das Ergänzen der Rechenoperationen im ersten unvollständigen Beispiel lediglich als unverbundenes Aufgabenformat wahrgenommen haben. Glen beschreibt daher nicht den Prozess der Bruchherstellung, sondern notiert die Ergänzung zu einem Ganzen.

Can wirkt bei der Bearbeitung des zweiten unvollständigen Beispiels hilflos und kann scheinbar weder einen Beitrag zur Erklärung der Bruchherstellung leisten, noch die Brüche in den vorgegebenen Figuren einzeichnen.

Zusammenfassend lassen sich die Interpretationen der Bearbeitungen von Can, Glen und Philip anhand der folgenden Deutungshypothesen zusammenfassen:

- Aufgrund einer oberflächlichen Verarbeitung des einleitenden Lösungsbeispiels werden die wesentlichen Aspekte des Bruchherstellungsverfahrens nicht erfasst und können in der Folge nicht auf das unvollständige Beispiel übertragen werden. Die Schüler haben das Lösungsbeispiel zu Beginn der Unterrichtsstunde eilig durchgesehen, es fand keine Kommunikation über den Inhalt statt und auch die fokussierenden Fragestellungen zu dem Lösungsbeispiel wurden nicht beachtet. Im Anschluss gehen sie umgehend zur Bearbeitung des ersten unvollständigen Beispiels über, in dem sie versuchen die zu ergänzenden Rechenoperationen herzuleiten. Das Pfeilschema zur Veranschaulichung der Bruchherstellung wird nicht auf das zweite unvollständige Beispiel übertragen.

- Es werden unterschiedliche Operationsvorstellungen zur Beschreibung der Bruchherstellung auf ikonischer und symbolischer Darstellungsebene angewandt. In den Bearbeitungen der Schüler ist zu erkennen, dass sie mit Bezug auf eine ikonische Darstellung die Teiloperatoren auf einer gegenständlichen Ebene eher mit einem Wegnehmen und Hinzufügen von Teilen verbinden, während sie diese mit Bezug auf eine symbolische Darstellung eher mit einem Teilen und Vervielfachen verbinden. In der Folge ergänzen sie im ersten unvollständigen Beispiel unterschiedliche Rechenoperation für die Herstellung desselben Bruchs. Im Kontext der ikonischen Darstellung von Brüchen hingegen interpretieren Glen und Philip die Teiloperatoren bzw. die Nenner und Zähler von Brüchen als Teilen des Ganzen in gleich große Teile und Färben der dem Zähler entsprechenden Anzahl dieser Teile. Die Schüler stellen keine Beziehung zwischen den Beschreibungen der Herstellungshandlung auf ikonischer und symbolischer Ebene her.

\section{Luca \& Miguel - Entkopplung von symbolischer und anschaulicher Ebene}

Luca und Miguels Ergebnisse im Vortest fallen mit lediglich $20 \%$ und 15\% der erreichbaren Punktzahl in das niedrigste Leistungsquartil. In ihrer Partnerarbeit überwiegen individuelle Arbeitsphasen und sie sprechen allgemein sehr wenig über 
die Bearbeitung der Aufgaben. Das Lösungsbeispiel zur Einführung in die Unterrichtsstunde haben sie eingehend gelesen und auch die fokussierenden Fragen zum Lösungsbeispiel bearbeitet.

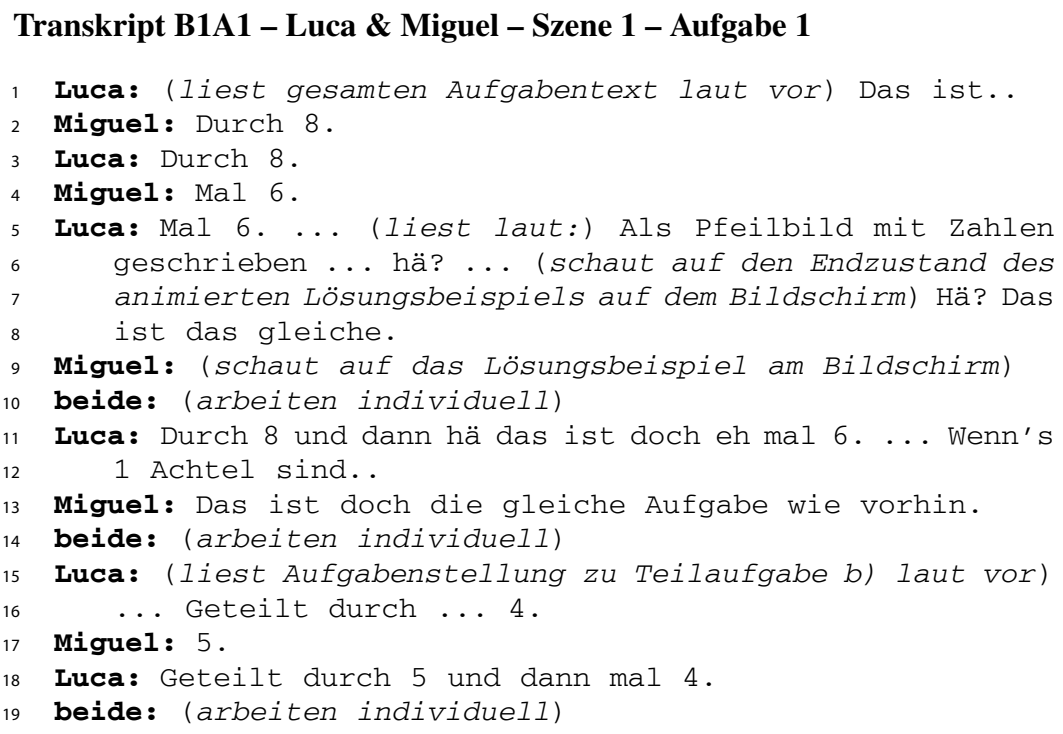

In ihrer Bearbeitung des ersten unvollständigen Beispiels nennen sie umgehend im Einvernehmen ,durch 8“ $(2,3)$ und ,mal 6“ $(4,5)$ für die Ergänzungen der Rechenoperationen im ikonisch dargestellten Pfeilschema zur Herstellung des Bruchs $\frac{6}{8}$. Beide Schüler nennen die Rechenoperationen, ohne dass sie lange darüber nachdenken oder diskutieren müssen. Luca ist zunächst irritiert davon, dass sie die gleichen Rechenoperationen erneut in der symbolischen Pfeilschemadarstellung ergänzen sollen: „Hä? Das ist das gleiche“ (7-8). Seine Irritation kann so interpretiert werden, dass er erkennt, dass an dieser Stelle die gleichen Rechenoperationen eingetragen werden müssen wie zuvor, und er entsprechend unabhängig von der Darstellung zuvor die gleichen Rechenoperationen identifiziert. Er vergewissert sich durch einen Blick auf den Endzustand des animierten Lösungsbeispiels auf dem Bildschirm, dass er dabei richtig liegt. Auch Miguel stellt fest: „Das ist doch die gleiche Aufgabe wie vorhin“ (12).

Nachdem sie ohne weiteren Kommentar die Rechenoperationen in ihrem Arbeitsheft ergänzt haben liest Luca die Aufgabenstellung zu Aufgabenteil b) laut vor, in 
der die Rechenoperationen zur Herstellung des Bruchs $\frac{4}{5}$ ergänzt werden sollen. Nach kurzem Nachdenken und Zögern nennt Luca ,geteilt durch ... 4“ (15) als erste Rechenoperation. Er wird umgehend von Miguel korrigiert, dass sie zuerst durch 5 teilen müssen (16) und nennt unmittelbar darauf den vollständigen Rechenweg ,geteilt durch 5 und dann mal 4“ (17). An dieser Stelle sind zwei Erklärungen für seinen Zahlendreher denkbar: Entweder er hat sich lediglich versprochen oder er hat die Bedeutung von Zähler und Nenner eines Bruchs vertauscht (vgl. auch Abb. 5.9).
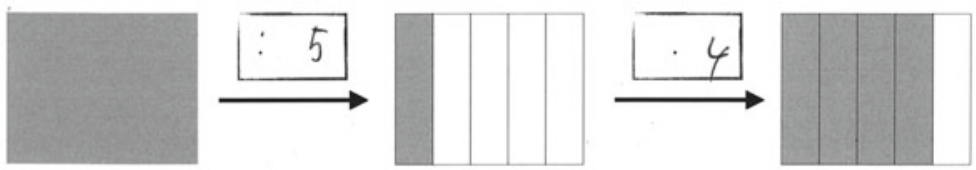

Als Pfeilbild mit Zahlen geschrieben:
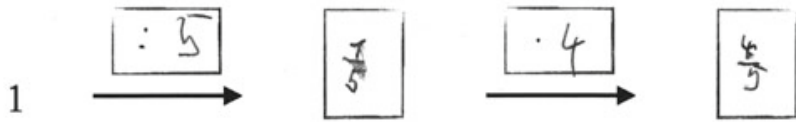

Abbildung 5.9 Lucas Lösung von Aufgabe 1 b)

\section{Transkript B1A2 - Luca \& Miguel - Szene 2 - Aufgabe 2}

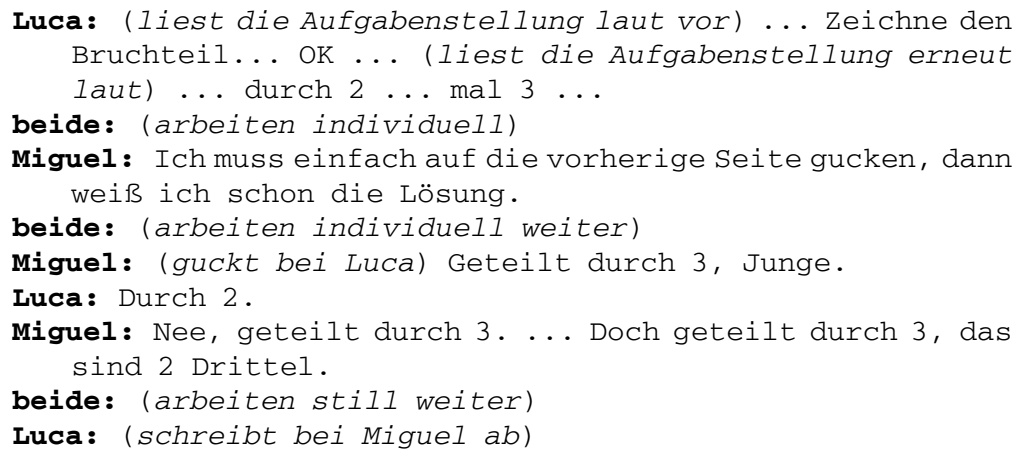




\section{Lösung:}

1)

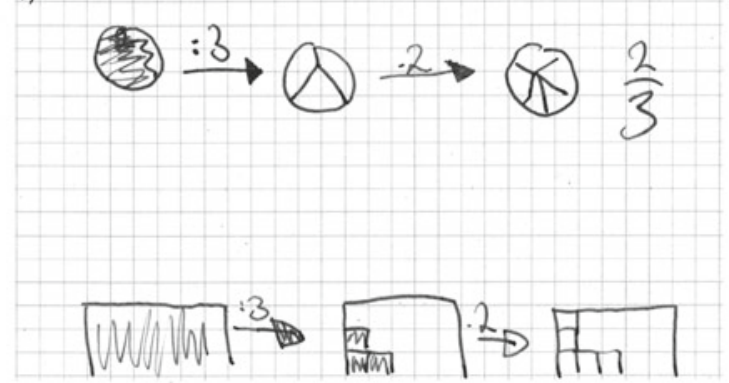

2)

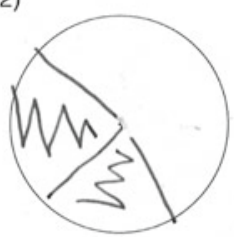

b) Jetzt ist das Ganze ein Rechteck.

1) Erkläre, wie $\frac{3}{8}$ des Ganzen entsteht. Du kannst auch ein Pfeilbild zeichnen.

2) Zeichne den Bruchteil ein.

\section{Lösung:}

1)

$$
\frac{3}{8}: 8=\frac{1}{8} \cdot 3=\frac{3}{8}
$$

2)

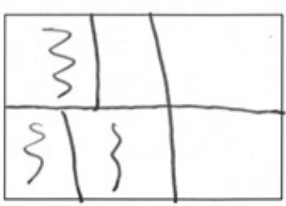

Abbildung 5.10 Miguels Lösung von Aufgabe 2

Nach der Bearbeitung des ersten unvollständigen Beispiels gehen sie unmittelbar zum zweiten unvollständigen Beispiel über. Nachdem Luca die Aufgabenstellung laut vorgelesen hat, nennt er seine Lösung ,durch 2 ... mal 3“ (3). Er macht erneut einen Zahlendreher und interpretiert den Zähler als Anzahl der Teile des Ganzen und den Nenner als Anzahl der Teile. Dieser wiederholte Zahlendreher stützt die Annahme, dass sein Zahlendreher bei der Bearbeitung des ersten unvollständigen Beispiels kein Versprecher war, sondern dass er tatsächlich die Bedeutung von Zähler und Nenner vertauscht. Nach einem Blick auf Lucas Arbeitsheft wird er von

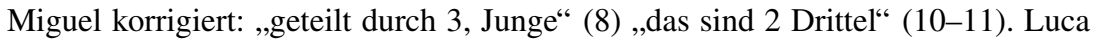
besteht zunächst weiterhin darauf, dass sie zunächst „,durch 2“(9) rechnen müssen, übernimmt aber schließlich doch die Lösung von Miguel (Abb. 5.10). Im weiteren Verlauf arbeiten Luca und Miguel individuell in Stillarbeit. Im Video ist zu erkennen, dass Luca die Lösungen seines Partners abschreibt. 
Die sprachliche Kommunikation von Luca und Miguel lässt annehmen, dass sie das Verfahren zur Bruchherstellung verstanden haben und jeweils die korrekten Teiloperatoren zur Herstellung der vorgegebenen Brüche ableiten und notieren können. Auch ihre schriftlichen Bearbeitungen zeigen, dass sie auf symbolischer Ebene das Operatorschema zur Herstellung von Brüchen übertragen und anwenden können.

Miguels ikonische Darstellungen der Bruchherstellungen deuten jedoch darauf hin, dass er die Rechenoperationen nicht mit den entsprechenden Herstellungshandlungen verknüpft bzw. seine ikonischen Darstellungen nicht die Rechenoperationen repräsentieren. Zur Darstellung des Bruchs $\frac{2}{3}$ zeichnet er zwei ikonische Pfeilschemata, eines mit einer Repräsentation als Kreis und eines mit einem Rechteck. Für die Darstellung in einem Kreis übersetzt er den Teiloperator :3 korrekt als Einteilen des Ganzen in drei gleich große Teile. In seiner Darstellung in einem Rechteck hingegen teilt er nicht die ganze Figur in drei gleich große Teile, sondern zeichnet lediglich drei gleich große Teile ein, die jedoch zusammen entsprechend der Kästchen auf dem Arbeitsblatt lediglich etwa $\frac{3}{15}$ des Ganzen Rechtecks entsprechen. Demgegenüber ist seine Übersetzung des zweiten Operators konsistent über die Darstellung in einem Kreis und einem Rechteck. In beiden Fällen übersetzt er den Teiloperator .2 als Hinzufügen von zwei weiteren Teilen. Während die zusätzlichen Teile, die er in das Rechteck einzeichnet, die gleiche Größe wie die zuvor eingezeichneten Teile haben, halbiert er zwei der zuvor eingezeichneten Segmente in seiner Kreisdarstellung, da er zuvor den ganzen Kreis in gleich große Teile eingeteilt hat. In beiden Darstellungen kann jedoch angenommen werden, dass er zwei Teile hinzufügt. In beiden Endzuständen seiner Darstellungen im Kreis und im Rechteck sind keine Segmente farbig markiert.

Seine ikonische Darstellung des Bruchs in der vorgegebenen Kreisfläche unterscheidet sich wiederum erneut von seiner Darstellung im Pfeilschema. Hier hat er den ganzen Kreis in drei unterschiedlich große Teile eingeteilt und zwei Teile markiert, deren Fläche in etwa gleich ist. Die markierten Teile entsprechen jedoch weniger als der Hälfte des ganzen Kreises.

In Aufgabenteil b), zeichnet er für die Erklärung der Herstellung von $\frac{3}{8}$ kein Pfeilschema, sondern einen ähnlichen Term, wie ihn auch Bennet und Julius notiert haben. Hier fällt jedoch auf, dass er seine Rechnung nicht mit 1 als symbolische Repräsentation eines Ganzen beginnt, sondern von dem Bruch $\frac{3}{8}$ ausgeht und schreibt: $\frac{3}{8}: 8=\frac{1}{8} \cdot 3=\frac{3}{8}$. Dabei benennt er die Herstellungsoperatoren $: 8$ und $\cdot 3$ jedoch korrekt. Für die ikonische Darstellung des Bruchs teilt er das ganze Rechteck in sechs unterschiedlich große Teile von denen er drei Teile markiert. Hier kann angenommen werden, dass er nicht darauf geachtet hat, wie viele Teile er einzeichnet und so lediglich sechs anstelle von acht Teilen eingezeichnet hat, von denen er aber dennoch drei Teile markiert. 
Insgesamt zeigen die schriftlichen Produkte von Miguel, die Luca abgeschrieben hat, dass er zwar die Operatoren zur Herstellung eines Bruchs korrekt angeben kann, jedoch die Rechenoperationen anders als vorgesehen interpretiert und entsprechend in fehlerhafte ikonische Darstellungen übersetzt. Er übersetzt den ersten Operator $: n$ eines Bruchs $\frac{m}{n}$ nicht als Teilen des Ganzen in $n$ gleich große Teile, sondern als Einzeichnen von $n$ drei gleich großen Teilen, die jedoch nicht das Ganze in gleiche Teile einteilen. Den zweiten Operator $\cdot m$ übersetzt er nicht mit dem Färben bzw. Markieren von $m$ Teilen, sondern als Hinzufügen von $m$ Teilen. Dieses Muster ist in Ansätzen auch beim Einzeichnen eines Bruchs in eine vorgegebene Figur zu erkennen. Auch hier teilt er das Ganze zwar in die entsprechende Anzahl von Teilen, jedoch sind die Teile nicht gleich groß.

Zusammenfassend lassen sich die folgenden Deutungshypothesen auf Grundlage der Bearbeitung von Luca und Miguel festhalten:

- Miguel kann zwar die Operatoren zur Bruchherstellung auf symbolischer Darstellungsebene korrekt auf die Herstellung von neuen Brüchen übertragen, jedoch verbindet er fehlerbehaftete anschauliche Wirkungen mit diesen, die letztendlich in falschen Darstellungen der Anteile resultieren. Es ist hier bereits eine Entkopplung von symbolischer und anschaulicher Ebene zu erkennen.

- Die Wirkung der Herstellungsoperatoren ist von der Repräsentation abhängig, auf die sie angewendet wird. In seiner Darstellung in einem Kreisdiagramm interpretiert Miguel die Division :3 korrekt als Teilen des Ganzen in drei nahezu gleich große Teile. In seiner Rechteckdarstellung dagegen deutet er die Division :3 als Einzeichnen von drei beliebigen untereinander gleich großen Teilen, anstelle das Ganze in drei gleiche Teile aufzuteilen.

\section{Vergleich der Bearbeitungen der unvollständigen Beispiele}

Auf Grundlage sachanalytischer Überlegungen wurden für die Bearbeitung der beiden unvollständigen Beispiele drei zentrale Transferprozesse identifiziert: Die Übertragung der Operatorstruktur, die Anwendung dieses Verfahrens auf die Herstellung neuer Brüche mitsamt ihren ikonischen Repräsentationen sowie die Übersetzung der Herstellungshandlung zwischen der ikonischen und symbolischen Repräsentationsebene.

\section{Übertragen der Operatorstruktur \& Anwenden des Verfahrens auf neue}

Brüche: In allen drei vorgestellten Lösungsprozessen kann ein Transfer des Operatorschemas zumindest in Ansätzen nachgewiesen werden. Hierbei ist zu bedenken, dass die Schülerinnen und Schüler in den zwei vorhergehenden Unterrichtsstunden die Stammbruchherstellung behandelt haben, weshalb der erste Teiloperator bzw. 
der erste Schritt des Bruchherstellungsverfahrens ihnen möglicherweise bekannt ist. Entgegen dieser Annahme kann beobachtet werden, dass die Erweiterung der Stammbruchherstellung um den zweiten Teiloperator zum Vervielfachen eines Teils eine neue Anwendungssituation darstellt, in der die Bedeutung des ersten Teiloperators neu interpretiert und ausgehandelt wird. In keiner der hier diskutierten Bearbeitungen wird ein Bezug zum vorhergehenden Unterricht hergestellt. Zudem konnte in zwei der betrachteten Fälle beobachtet werden, dass die Erweiterung des Verfahrens um den zweiten Teiloperator der Überinterpretation des Schemas führte, und angenommen wurde, dass auch zur Herstellung eines Bruchs $\frac{1}{n}$ zwei Operatoren benötigt werden: $: n$ und $\cdot 1$.

Die allgemeine Grundstruktur der Hintereinanderausführung von zwei Teiloperatoren wird von allen Schülern erkannt und übertragen. Es treten jedoch Fehler bzw. Schwierigkeiten bei der Anwendung der Teiloperatoren zur Herstellung neuer Brüche auf, die auf die Übersetzung der Herstellungshandlung zwischen der symbolischen und anschaulichen Repräsentationsebene zurückgeführt werden können.

\section{Übersetzen der Herstellungshandlung zwischen der ikonischen und symboli-} schen Repräsentationsebene: Die Übersetzung zwischen der anschaulichen und symbolischen Darstellungsebene stellt sich in allen hier dargestellten Bearbeitungsprozessen sehr unterschiedlich dar und führt zu Schwierigkeiten unterschiedlicher Art. Bei einer reinen Betrachtung der schriftlichen Lösungen der Schülerinnen und Schüler würden alle beobachteten Fehler dem Fehlermuster „Wahl der falschen Rechenoperation“ (vgl. Abschnitt 2.2.4) zugeordnet werden. Die Analyse der Bearbeitungsprozesse zeigt jedoch in allen drei Fällen deutliche Unterschiede in den Ursachen für diese Fehler.

Julius Interpretation der Herstellungsoperatoren orientiert sich an Handlungen mit konkreten Gegenständen. Entsprechend der Formulierung „,man teilt das Ganze in $n$ gleiche Teile und nimmt davon $m$ " interpretiert er den zweiten Teiloperator subtraktiv als „Wegnehmen von Teilen“. Aus diesem Grund argumentiert er dafür, dass der zweite Teiloperator ,minus m“ sein müsse. Im Gegensatz dazu interpretiert sein Partner Bennet die Formulierung in einem multiplikativen Zusammenhang als Vervielfachen eines Teils.

In der Bearbeitung von Can, Glen und Philip konnte beobachtet werden, dass sie zwar Bezug auf die grundlegende Struktur von zwei Herstellungsschritten genommen haben, jedoch die Bedeutung der jeweiligen Teiloperatoren anhand der vorgegebenen ikonischen Darstellungen abgeleitet haben ohne einen Bezug zum Lösungsbeispiel oder den vorhergehenden Unterrichtsstunden herzustellen. In ihren Ableitungen der Rechenoperationen für die einzelnen Herstellungsschritte ist zu beobachten, dass sie abhängig von der Repräsentationsebene, auf der sie argumentieren, 
unterschiedliche Operationsvorstellungen anwenden. Während sie auf der ikonischen Repräsentationsebene die Teiloperatoren mit einem Wegnehmen und Hinzufügen von Teilen interpretieren, beschreiben sie auf symbolischer Ebene ein Teilen und Vervielfachen. Als Folge erklären sie die Herstellung desselben Bruchs mit unterschiedlichen Teiloperatoren und somit mit unterschiedlichen Herstellungshandlungen.

Miguel gelingt es sowohl auf ikonischer wie auch auf symbolischer Ebene die jeweils korrekten Teiloperatoren zur Herstellung der verschiedenen Brüche abzuleiten. Seine ikonischen Darstellungen der Bruchherstellung bringen jedoch zum Vorschein, dass er die Teiloperatoren mit fehlerbehafteten anschaulichen Wirkungen verbindet, die zudem abhängig von dem verwendeten Repräsentationsobjekt sind. Anhand einer Kreisrepräsentation übersetzt er den ersten Teiloperator korrekt mit dem Einteilen der ganzen Figur in gleich große Teile. Bei der Darstellung in einer Rechteckdarstellung hingegen veranschaulicht er den ersten Teiloperator als Einzeichnen von Teilen, deren Größe unabhängig vom Ganzen ist und deren Anzahl der Zahl im Nenner des darzustellenden Bruchs entspricht. Den zweiten Teiloperator interpretiert er als Hinzufügen einer Anzahl von Teilen, die er dem Zähler des darzustellenden Bruchs entnimmt. In seiner Kreisrepräsentation fügt er zwei Teile hinzu, indem er zwei der zuvor eingeteilten Kreissegmente halbiert. Demgegenüber zeichnet er in seiner Rechtecksrepräsentation zwei zusätzliche Teile ein.

Insgesamt ist festzustellen, dass in allen beschriebenen Fällen ein Transfer der grundlegenden Operatorstruktur als Hintereinanderausführung von zwei Teiloperatoren identifiziert werden kann. Dabei kann es jedoch zu fehlerhaften anschaulichen Interpretationen der Wirkungen dieser Teiloperatoren kommen, was zur Wahl der falschen Rechenoperation oder zur fehlerhaften ikonischen Darstellung der Anteile führt. In diesen Fällen ist somit in einem sehr frühen Stadium der Begriffsentwicklung eine Entkopplung von symbolischer und anschaulicher Ebene zu erkennen.

\subsubsection{Bruchdarstellung an einer Strecke}

Während die ikonische Darstellung von Brüchen bis zu diesem Zeitpunkt nahezu ausschließlich anhand von Kreis- und Rechtecksrepräsentationen vorgenommen wurde, sollen die Lernenden in dieser Transferaufgabe ihr Vorgehen auf die lineare Darstellung einer Strecke übertragen. Für jede Teilaufgabe ist eine Strecke mit der Länge von zwölf Kästchen abgebildet, an denen die Lernenden die Brüche $\frac{1}{4}, \frac{2}{3}, \frac{5}{6}$ und $\frac{5}{12}$ darstellen sollen (siehe Abb. 5.11). Es ist somit ein Transfer auf Repräsentationsebene erforderlich, der in zwei Transferschritten beschrieben werden kann: 
- Die Übertragung des Teiloperators : $n$ zur Einteilung der Strecke in $n$ gleich große Teile bzw. Abschnitte und

- die Übertragung des Teiloperators $\cdot m$ zum Färben von $m$ Streckenabschnitten.

Für diese Aufgabe wurde bewusst eine Streckenabbildung gewählt, die eine Einteilung ohne Berechnungen auf Zahlenebene ermöglicht. Auf diese Weise sollen die Lernenden sich auf die ikonische Darstellung konzentrieren können. Mit dem Repräsentationstransfer zur Darstellung von Brüchen an einer Strecke soll die Verallgemeinerung des Verfahrens zur Bruchdarstellung unterstützt werden, indem es von den bekannten Repräsentationsobjekten Kreis und Rechteck losgelöst wird. Aus didaktischer Perspektive ist dies ein wichtiger Schritt für die flexible Anwendung des Verfahrens auf unterschiedliche Bezugsgrößen und Darstellungen.

\section{Aufgabe 4}

Ein Ganzes kann auch durch eine Strecke dargestellt werden.

Färbe die Bruchteile der Strecke.

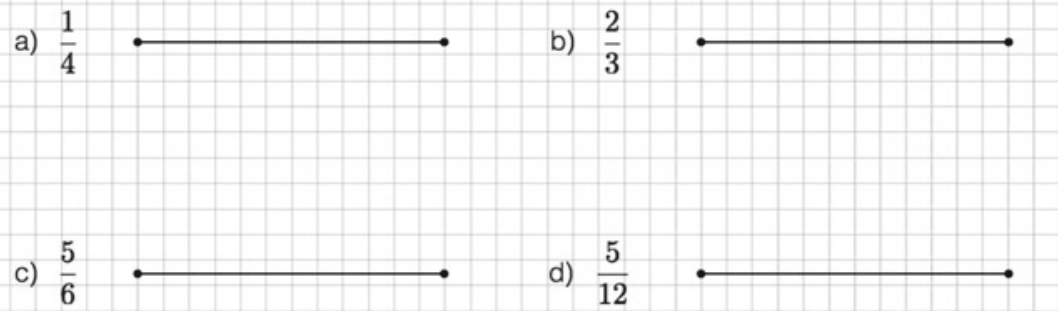

Abbildung 5.11 Aufgabe 4

Der Vortest enthielt ein Item zum Ablesen von Brüchen aus einer Streckendarstellung sowie zwei Items zur Darstellung von Brüchen in einer Strecke. Nur vier der 30 Lernenden konnten im Vortest den Bruch $\frac{3}{4}$ in einer in vier Teile geteilten Strecke ablesen. Demgegenüber konnten etwa ein Drittel der Lernenden die Brüche $\frac{3}{4}$ und $\frac{3}{8}$ in einer in acht gleiche Teile eingeteilten Strecke markieren.

\section{Bennet \& Julius - Übertragung der Operatorstruktur}

Im Anschluss an die Bearbeitung der unvollständigen Beispiele zeichneten Bennet und Julius ohne Schwierigkeiten die Bruchdarstellungen in Aufgabe 3. Dabei arbeiteten sie weitgehend in Einzelarbeit, wählten eigene Darstellungen und Eintei- 
lungen für das Ganze und verglichen ihre Zeichnungen. Während der Bearbeitung der Aufgabe stellten sie fest, dass die Darstellung in einem Kreis aufwändiger als die Darstellung von Bruchteilen in einem Rechteck sei, da ein Rechteck schneller und einfacher zu zeichnen sei und mit höherer Genauigkeit in gleich große Teile eingeteilt werden können. Direkt im Anschluss lesen die die Aufgabenstellung zu Aufgabe 4 und beginnen mit der Bearbeitung.

\section{Transkript B1A4 - Bennet \& Julius - Szene 1 - Aufgabe 4}
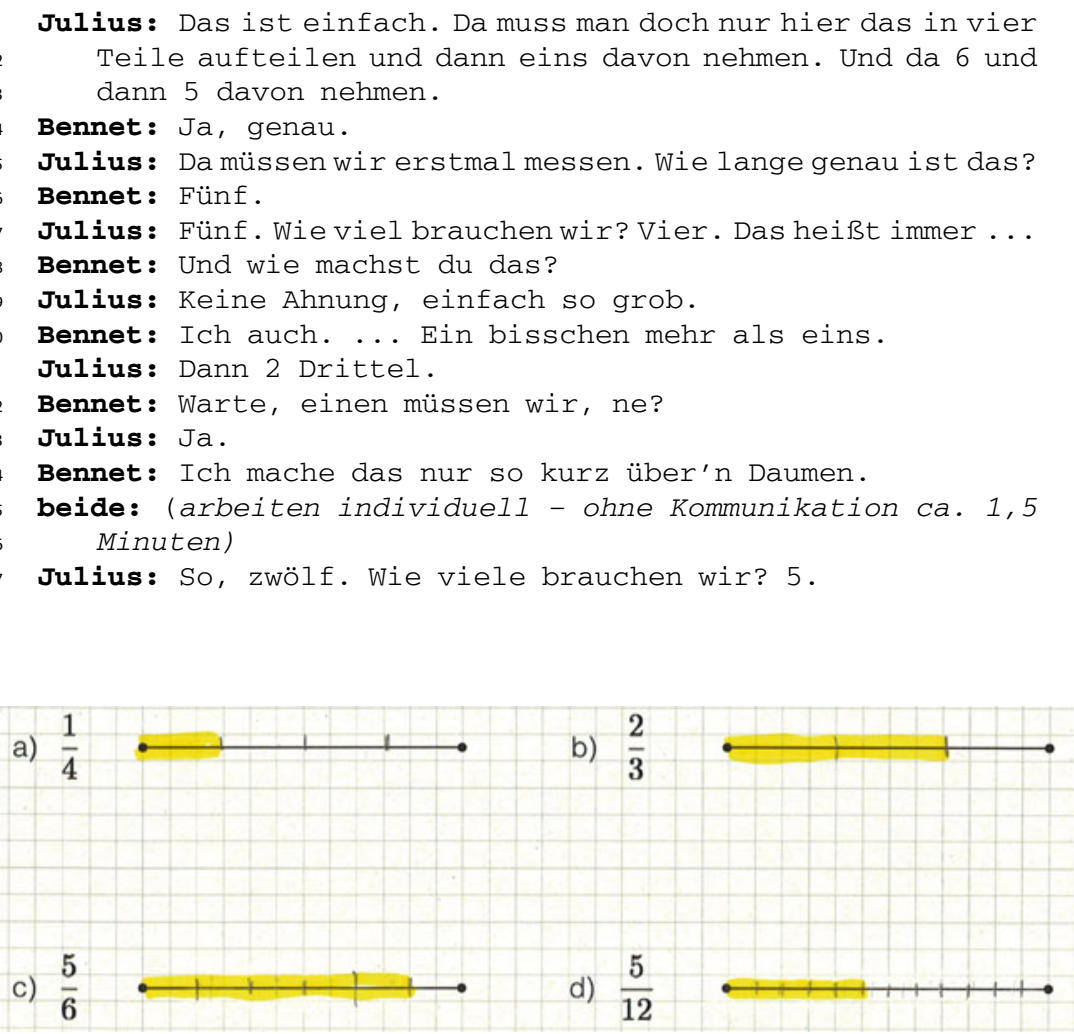

Abbildung 5.12 Julius Bearbeitung von Aufgabe 4

Julius kommentiert unmittelbar nach dem Lesen der Aufgabenstellung, dass die Darstellung von Bruchteilen an einer Strecke ,einfach“ sei, denn für die Darstellung 
des Bruchs $\frac{1}{4}$ in Teilaufgabe a) müsse ,,man doch nur hier das in vier Teile aufteilen und dann eins davon nehmen“ (1-2). Analog formuliert er seinen Lösungsvorschlag zur Darstellung des Bruchs $\frac{5}{6}$ in der unter a) gelegenen Teilaufgabe c): „Und da 6 und dann 5 davon nehmen" (2-3). Bennet stimmt ihm zu, dass dies ein richtiges Vorgehen sei (4). Zur Bestimmung der Größe eines Teils schlägt Julius vor, dass man zunächst die Länge der ganzen Strecke „genau“ vermessen müsse (5), was Bennet auch umgehend tut und antwortet, dass die ganze Strecke die Länge ,5“ habe (6). Eine Maßeinheit nennt er dabei nicht. Julius unterteilt in der Folge die Strecke in 4 gleich große Teile. Bennet scheint Schwierigkeiten zu haben die 5 Einheiten lange Strecke in vier gleiche Teile zu teilen und fragt Julius wie er das mache (8). Julius antwortet ihm, dass er nicht wisse, wie man die Strecke in exakt gleich große Segmente teilt und er die Teilung „grob“ vornimmt (9). Bennet nimmt dieses Vorgehen auf und schätzt die Länge eines Teils als ,ein bisschen mehr als eins“ (10). Während Julius bereits zu Teilaufgabe b) übergeht (11), versichert sich Bennet bei Julius, dass in Teilaufgabe a) eines der vier Streckensegmente eingefärbt werden muss (12), was Julius ihm bestätigt (13).

Bennet erklärt, dass er ,das nur so kurz über'n Daumen mache“ (14), worauf eine individuelle Arbeitsphase folgt, in der beide Schüler die übrigen Aufgaben bearbeiten.

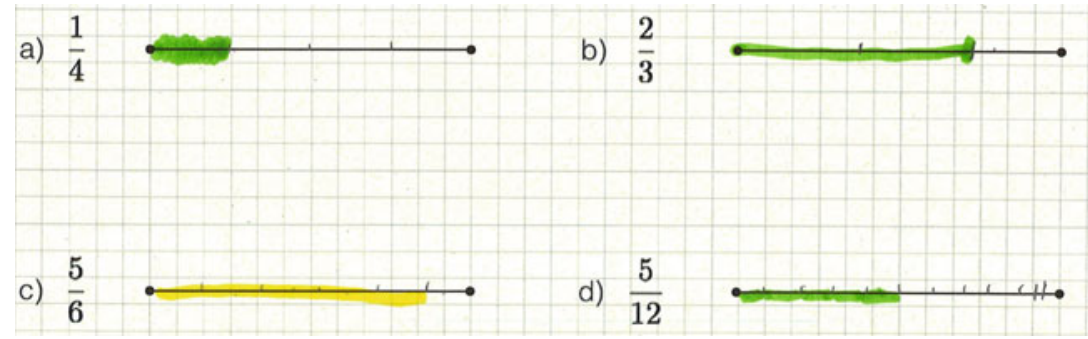

Abbildung 5.13 Bennets Bearbeitung von Aufgabe 4

Was Bennet mit seiner Bemerkung meint, wird erst bei der Betrachtung seiner Zeichnung ersichtlich. Im Gegensatz zu Julius, der, obgleich er es nicht sagt, die Kästchenstruktur des Arbeitsblattes als Hilfe nutzt, unterteilt Bennet die Strecke nach Augenmaß in die entsprechende Anzahl von Teilen bzw. Segmenten.

Zum Ende der individuellen Arbeitsphase ist Julius bei Teilaufgabe d) angekommen und spricht laut bei seiner Bearbeitung mit. Er teilt die Strecke in zwölf 
gleich große Teile und fragt wie viele sie „brauchen“. Er beantwortet seine Frage unmittelbar selbst mit ,5“ (17).

Die Analyse des Bearbeitungsprozesses zeigt, dass Bennet und Julius Bruchteile ohne erkennbare Schwierigkeiten an einer Strecke darstellen können. In Julius Erklärungen ist die Übertragung der Herstellungshandlung deutlich zu erkennen. Er beschreibt, dass man die Strecke bzw. das Ganze zur Darstellung der Brüche $\frac{1}{4}$ und $\frac{5}{6}$ zunächst in vier (bzw. sechs) gleiche Teile aufteilen und dann einen (bzw. fünf) Teile „davon nehmen“ (1-3) müsse. Mit ,nehmen“ bezieht er sich an dieser Stelle auf ein Markieren des entsprechenden Anteils, wobei seine Formulierung weiterhin eine Orientierung an Handlungen mit konkreten Darstellungsobjekten erkennen lässt. Ungeachtet dessen ermöglicht ihm der Rückgriff auf die Operatorstruktur die korrekte Darstellung ohne erkennbare Schwierigkeiten.

Die Leichtigkeit und Direktheit, mit der er die zur Darstellung erforderlichen Schritte nennt, deutet vor dem Hintergrund seiner Schwierigkeiten bei der Bearbeitung der unvollständige Beispiele darauf hin, dass er die Herstellungshandlung wohl verstanden hat und flexibel auf neue Brüche und Darstellungsobjekte übertragen kann. Anders als in den unvollständigen Beispielen sind hier jedoch keine symbolischen Rechenoperatoren zu nennen, weshalb aus dieser Bearbeitung keine weiteren Schlüsse auf die Entwicklung der Operatorvorstellung gezogen werden können.

Um eine möglichst genaue Einteilung der Strecke vornehmen, möchte Julius die Länge der Strecke messen, was Bennet für ihn übernimmt. Die metrische Länge von $5 \mathrm{~cm}$ ermöglicht jedoch kein ganzzahliges Teilen in vier Teilabschnitte, wie sie in Teilaufgabe a) benötigt werden, sodass beide Schüler eine andere Methode zur Einteilung der Strecke wählen. Während die Zeichnungen von Julius (vgl. Abb. 5.12) nahelegen, dass er im Weiteren die unterliegende Kästchenstruktur des Arbeitsblattes zur Einteilung der Strecke benutzt, teilt Bennet die Streckensegmente nach Augenmaß ein. Er benutzt dazu das geschätzte Maß von ,ein bisschen mehr als

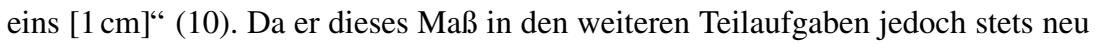
abschätzen muss, deuten seine Zeichnungen (vgl. Abb. 5.13) darauf hin, dass er in den weiteren Teilaufgaben nicht die Länge eines Teils überschlägt, sondern per Augenmaß die Länge eines Teils im Verhältnis zur ganzen Strecke und der Anzahl der benötigten Teilen abschätzt. Dies wird besonders in seinen Zeichnungen zu den Teilaufgaben b) und d) deutlich, bei denen er die Einteilung zunächst regelmäßig vornimmt, zum Ende hin aber nicht mehr genug Platz für die entsprechende Anzahl von Teilen vorhanden ist und er diese entsprechend kleiner einzeichnet. Er erklärt, dass er „das nur so kurz über'n Daumen“ (14) mache. Dieses Vorgehen deutet darauf hin, dass es ihm nicht wichtig ist, dass die Einteilung exakt ist, sondern lediglich die Grundstruktur erkennbar sein muss. In diesem Zusammenhang kann sein Vorgehen 
als Zeichen dafür interpretiert werden, dass er eine zunehmende Flexibilität bei der Darstellung von Brüchen entwickelt.

Insgesamt kann die Analyse der Bearbeitung von Bennet und Julius in folgenden Deutungshypothesen zusammengefasst werden:

- Bennet und Julius übertragen das Bruchherstellungsverfahren zur Darstellung von Brüchen an einer Strecke. Dabei gehen sie nach demselben Handlungsschema vor wie bei der Darstellung von Bruchteilen an einem Kreis oder einem Rechteck. Sie teilen die Strecke zunächst in gleich große Teilsegmente und färben den entsprechenden Anteil der Strecke. Die Übertragung der Herstellungsschritte führt dazu, dass der Repräsentationstransfer zur Strecke für Bennet und Julius keine Schwierigkeit darstellt und spontan vorgenommen wird.

- Julius Erklärungsmodell zur Bruchherstellung orientiert sich weiterhin an Handlungen mit konkreten Repräsentationsobjekten. Er formuliert erneut, dass das Ganze in gleiche Teile eingeteilt wird, von denen man im Anschluss eine bestimmte Anzahl ,nimmt“. Auf ikonischer Darstellungsebene interpretiert er das Nehmen von Teilen jedoch nicht als ein Wegnehmen, sondern als ein Färben bzw. Markieren einer bestimmten Anzahl von Teilen.

- Bennet löst sich zunehmend von einer Einteilung in exakt gleich große Teile. Stattdessen ist in seinen Darstellungen zu erkennen, dass er die Länge der Streckensegmente nicht rechnerisch oder abzählend bestimmt, sondern ihre Größe abschätzt und somit das Ganze nach Augenmaß in die entsprechende Anzahl von Teile zerlegt. Hierbei legt er keinen Wert auf eine exakte Darstellung, sondern auf die allgemeine Identifizierbarkeit der dargestellten Bruchteile, was auf eine zunehmende Verallgemeinerung und Abstraktion des Verfahrens hindeutet.

\section{Aliya und Aisha - Statische und dynamische Sichtweise auf Anteile}

Aliya und Aisha sind ein sehr leistungsheterogenes Schülerinnenpaar. Während Aliya zu den Klassenbesten gehört und im Vortest bereits $85 \%$ der Aufgaben korrekt lösen konnte, zählt Aishas Vortestergebnis mit lediglich $15 \%$ zu den niedrigsten Ergebnissen in der Lerngruppe. In der vorhergehenden Partnerarbeit ist zu erkennen, dass beide Schülerinnen sehr sorgfältig und gewissenhaft arbeiten. Sie haben das Lösungsbeispiel zur Einführung ausführlich gelesen und bereits während des Lesens die einzelnen Lösungsschritte in eigenen Worten erklärt. Auch die unvollständigen Beispiele haben sie sicher gelöst. Obgleich Aliya in der Interaktion der beiden Schülerinnen sehr dominant ist, stellt Aisha regelmäßig Zwischenfragen, bittet um eine Erklärung und macht selber Lösungsvorschläge, die zu einer erfolgreichen Aufgabenlösung beitragen. Die ikonischen Darstellung der Bruchteile in Aufgabe 3 fertigen beide Schülerinnen größtenteils in Einzelarbeit an und verglei- 
chen abschließend ihre Zeichnungen. Für die Darstellung der Brüche nutzen sie ausschließlich die Repräsentation eines Rechtecks und stellen alle Brüche korrekt dar.

\section{Transkript B1A4 - Aisha \& Aliya - Szene 1 - Aufgabe 4}

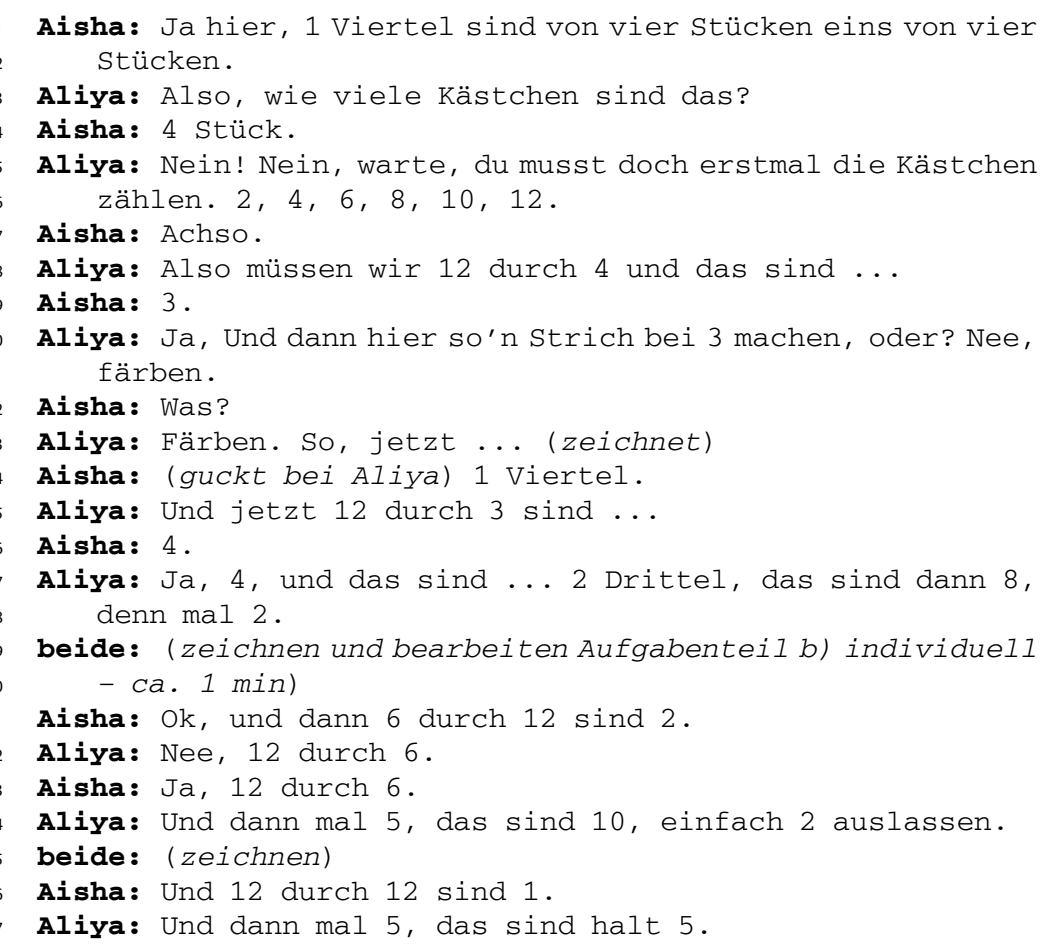

Direkt nachdem die beiden Schülerinnen die Aufgabenstellung gelesen haben beschreibt Aisha ihre Vorstellung von $\frac{1}{4}$ : „1 Viertel sind von vier Stücken eins von vier Stücken“ (1-2). Entsprechend ihrer Beschreibung stellt sie sich unter $\frac{1}{4}$ einen Anteil vor: Ein Teil von insgesamt vier Teilen. Aliya fragt sie darauf hin, was diese Feststellung für die ikonische Darstellung an der Strecke bedeutet: „Also, wie viele Kästchen sind das?“ (3). Sie fragt direkt nach der Länge der zu markierenden Strecke, wobei sie die der Strecke unterliegende Kästchenstruktur als Maß für die Länge definiert. Aisha antwortet ihr darauf, dass der Bruchteil $\frac{1}{4}$ „4 Stück“ (4), also vier Kästchen entspreche. Als Hintergrund für diesen Lösungsvorschlag kann 
die verbreitete Fehlvorstellung zu Stammbrüchen $\frac{1}{n}$ bezeichnet eine Menge von $n$ Objekten (vgl. Abschnitt 2.2.4) vermutet werden. Sie nimmt keinen Bezug zu der Länge der gesamten Strecke, sondern schließt vom Nenner des Bruchs direkt auf die Anzahl der zu färbenden Kästchen. Es anzunehmen, dass dieser Lösungsvorschlag von einer statisch-abbildhafte Vorstellung von $\frac{1}{4}$ herrührt, bei der sich eine diskrete Menge von vier Objekten vorstellt und keine Beziehung zwischen einem Teil und dem Ganzen herstellt. Anstelle die Anzahl der Kästchen zu bestimmen, die die Länge der ganzen Strecke ausmacht, schließt sie direkt auf die Anzahl von vier Kästchen. Entsprechend assoziiert sie den Bruch nicht mit seiner Herstellungshandlung, sondern als statisches Objekt.

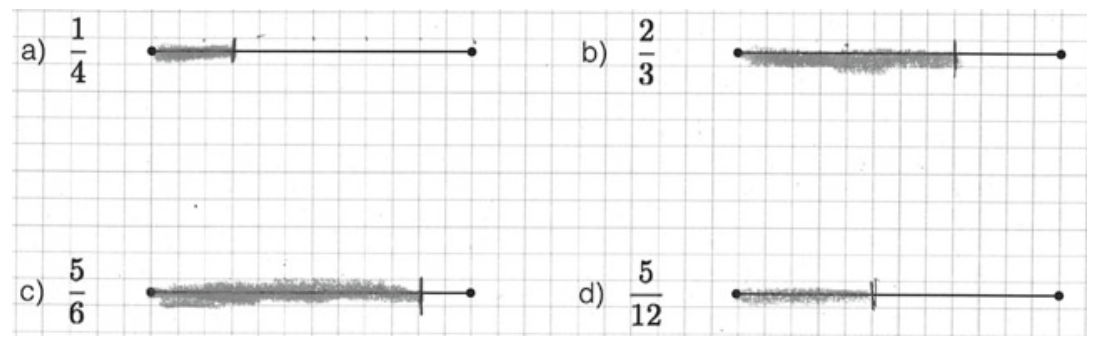

Abbildung 5.14 Aliyas Bearbeitung von Aufgabe 4

Aliya weist sie umgehend auf ihren Fehler hin: „Nein! Nein, warte, du musst doch erstmal die Kästchen zählen. 2, 4, 6, 8, 10, 12.“ (5-6). Sie erklärt damit, dass sie zunächst einmal die Länge der Strecke als Anzahl von Kästchen bestimmen müssen, die sie dann auch direkt in Zweierschritten abzählt. Sie erklärt weiter, dass sie nun die Länge der ganzen Strecke in vier gleiche Teile teilen müssen, was mit dem Kästchenmaß der Division „12 durch 4“ (8) entspricht. Aisha ergänzt, dass das Ergebnis dieser Rechnung „3“ (9) sei, worauf Aliya erklärt, dass sie folglich „hier so'n Strich bei 3 machen" (10-11) müssen, womit sie meint, dass sie am nach dem dritten Kästchen einen Trennstrich einzeichnen und die Teilstrecke von Beginn der Strecke bis zu diesem Trennstrich farbig markieren sollen. Im Gegensatz zu ihrer Partnerin ist in den Ausführungen von Aliya zu erkennen, dass sie eine dynamische Vorstellung zum Bruch $\frac{1}{4}$ aktiviert und einen Bezug zur Operatorstruktur des Bruchherstellungsverfahrens herstellt. Sie bestimmt die Länge der ganzen Strecke in der Einheit 1 Kästchen, teilt diese Strecke auf symbolischer Ebene mit dem Teiloperator $: 4$ in vier gleich große Teile, um dann einen Teil farbig zu kennzeichnen (Abb. 5.14). 
Aisha kommentiert diese Korrektur ihrer Partnerin mit „achso“ (7) und erweckt den Eindruck, dass sie ihren Fehler erkennt. Sie sieht ihrer Partnerin dabei zu, wie diese den Bruchteil markiert.

Aliya fährt umgehend mit der nächsten Teilaufgabe fort, in der sie den Bruch $\frac{2}{3}$ an der Strecke darstellen sollen. Dazu rechnet sie „12 durch 3“ (15) sind „4, und das sind ... 2 Drittel, dass sind dann acht, denn mal 2“ (17-18). Auch in dieser Rechnung ist deutlich zu erkennen, dass Aliya zur Bestimmung des Bruchteils die Operatorstruktur anwendet und zunächst :3 und dann $\cdot 2$ rechnet.

Nachdem die beiden Schülerinnen auch diesen Anteil der Strecke gekennzeichnet haben übernimmt Aisha Teilaufgabe c), in der der Bruchteil $\frac{5}{6}$ dargestellt werden soll. Dazu rechnet sie „6 durch 12 sind 2“ (21), wobei ungeachtet des Zahlendrehers zu erkennen ist, dass sie Aliyas Vorgehen aufnimmt und auf symbolischer Ebene den Operator :6 auf die Länge der Strecke anwendet und richtig feststellt, dass ein Teil die Länge von zwei Kästchen hat. Aliya ergänzt den zweiten Operator und rechnet: ,und dann mal 5, das sind 10, einfach 2 auslassen“ (24). Auch hier stellt sie unmittelbar einen Bezug zum Ganzen her, indem sie nicht ihrer Rechnung entsprechend zehn Kästchen vom Beginn der Strecke abzählt, um diese durch einen Trennstrich zu kennzeichnen, sondern feststellt, dass sie einfach von hinten zwei Kästchen abtrennen können, da die Strecke insgesamt zwölf Kästchen lang ist.

Auch für die letzte Teilaufgabe, der Darstellung des Bruchteils $\frac{5}{12}$ nutzen die beiden Schülerinnen die Operatorstruktur und rechnen „12 durch 12 sind 1“ (26) „und dann mal 5, das sind halt 5“ (27). Obgleich sie hier direkt die Kästchen abzählen könnten, formulieren sie ihr Vorgehen weiterhin anhand der zwei Teiloperatoren der Bruchherstellung und formulieren eine Rechnung auf symbolischer Ebene.

Zuletzt muss festgestellt werden, dass Aisha lediglich die Anwendung des ersten Teiloperators übernimmt und Aliya die Rechnung durch die Anwendung des zweiten Teiloperators vervollständigt. Obwohl angenommen werden könnte, dass Aisha nur einen Teil des Operatorschemas anwenden kann, enthält die Interaktion der beiden Schülerinnen keinen Hinweis darauf, dass Aisha möglicherweise Schwierigkeiten bei der Anwendung des zweiten Teiloperators hat. Stattdessen ist die Aufteilung der Rechenschritte zwischen den beiden Schülerinnen eher im Rahmen einer arbeitsökonomischen und interaktiven Partnerarbeit zu interpretieren.

Zusammenfassend kann der Bearbeitungsprozess von Aisha und Aliya anhand der folgenden Deutungshypothesen beschrieben werden:

- Aishas statische Deutung des Bruchs als Anteil einer diskreten Menge führt dazu, dass sie den Bruchteil nicht in Beziehung zum Ganzen sieht, sondern den Nenner des Bruchs als Anzahl der zu markierenden Längeneinheiten (Kästchen) 
interpretiert. Sie stellt zunächst keinen Bezug zum Bruchherstellungsverfahren her.

- Im Gegensatz zu ihrer Partnerin interpretiert Aliya den Bruch dynamisch vor dem Hintergrund des Bruchherstellungsverfahrens. Sie überträgt die Operatorstruktur als Hintereinanderausführung von zwei Teiloperatoren und berechnet in der Folge die Länge der Bruchteile der Strecke auf symbolischer Ebene.

- Auf ihren Fehler hingewiesen, scheint Aisha ihr fehlerhaftes Denkmuster zu erkennen und das Vorgehen ihrer Partnerin zu übernehmen, sodass für die nachfolgenden Bruchteile das Operatorschema anwendet und die Partnerinnen kooperativ die Längen der weiteren Streckenanteile berechnen.

\section{Laura \& Nike - Unvollständige Übertragung des Bruchherstellungsverfahrens} Die Vortestergebnisse von Laura und Nike können mit 40\% (Laura) und 65\% (Nike) den mittleren Leistungsstufen zugeordnet werden. Zum Einstieg in die Unterrichtsstunde haben sie das animierte Lösungsbeispiel eingehend gelesen und bereits während des Lesens über die einzelnen Herstellungsschritte gesprochen und diese nachvollzogen. Sie hatten keine Schwierigkeiten die fokussierenden Fragestellungen zum Lösungsbeispiel zu beantworten und in ihren Bearbeitungen der unvollständigen Beispiele war deutlich zu erkennen, dass sie die Operatorstruktur erfolgreich auf neue Brüche anwenden. In einer ikonischen Darstellung des Bruchs $\frac{3}{8}$ im zweiten unvollständigen Beispiel haben sie gar versucht, den Herstellungsprozess in ihre Bruchdarstellung zu integrieren (vgl. Abb. 5.15).

Abbildung 5.15 Nikes

Darstellung des Bruchs $\frac{3}{8}$ im zweiten unvollständigen

Beispiel

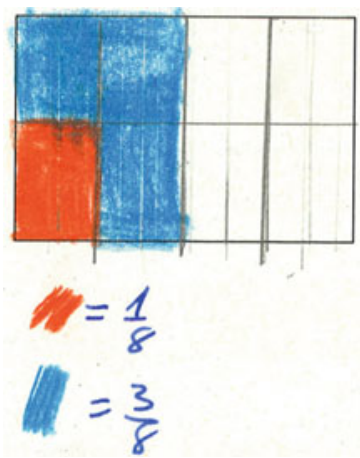




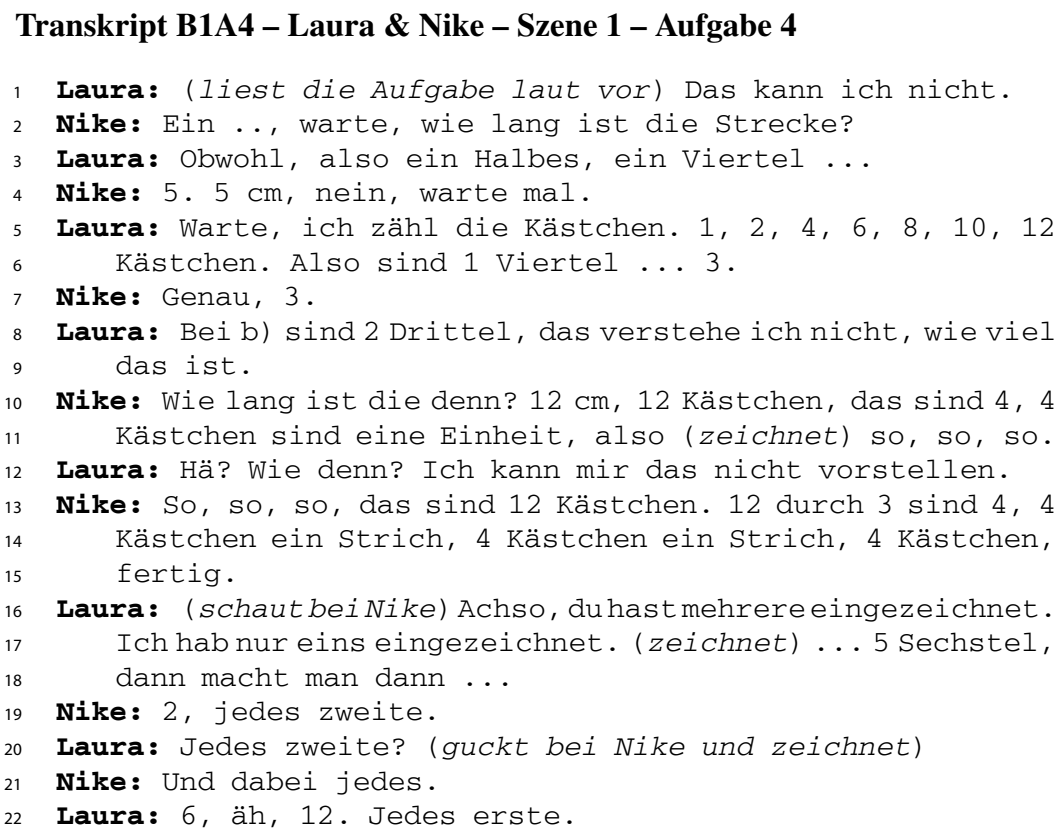

Laura erklärt unmittelbar nachdem sie die Aufgabenstellung laut vorgelesen hat: „Das kann ich nicht“ (1), relativiert dann jedoch nachdem sie kurz nachgedacht hat und ihre Partnerin Nike sie nach der Länge der vorgegebenen Strecke gefragt hat (2), dass sie womöglich doch eine Idee habe: „Obwohl, also ein Halbes, ein Viertel ...“ (3). Ihre Äußerung deutet darauf hin, dass sie in Gedanken die Strecke sukzessive halbiert, sodass sie „ein Halbes, ein Viertel“ (3) erhält. Es wird nicht ersichtlich, inwieweit die Nachfrage ihrer Partnerin sie auf diese Idee gebracht hat. Es ist durchaus möglich, dass sie mit ihrer anfänglichen Bemerkung, dass sie das nicht könne, zum Ausdruck bringt, dass sie keinen spontanen Zugang bzw. keinen Lösungsansatz erkennt. Die Nachfrage ihrer Partnerin richtet ihre Aufmerksamkeit dann jedoch darauf, dass der Anteil der Strecke in Form einer Länge bestimmt werden müsse, wozu zunächst die Länge der ganzen Strecke bestimmt werden muss. Diese Interpretation würde bedeuten, dass ihr für einen Ansatz die entsprechende Größe bzw. ein $M a ß$ für die Einteilung der Strecke fehlt.

Während Nike mit ihrem Lineal die Länge der Strecke ausmisst (4), beginnt Laura die Länge der Strecke durch Abzählen der Kästchen als Einheit zu bestimmen: „Warte, ich zähl die Kästchen. 1, 2, 4, 6, 8, 10, 12 Kästchen“ (5). Sie teilt die Anzahl 
der Kästchen durch vier und stellt dann fest: „Also sind 1 Viertel ... 3“ (5-6). Sie wendet entsprechend den Operator :4 auf die Anzahl der Kästchen an, um $\frac{1}{4}$ zu erhalten. Ihr Partnerin Nike stimmt ihr zu (7) und Laura geht direkt zu Aufgabenteil b) über, in der der Bruch $\frac{2}{3}$ an der Strecke dargestellt werden soll.

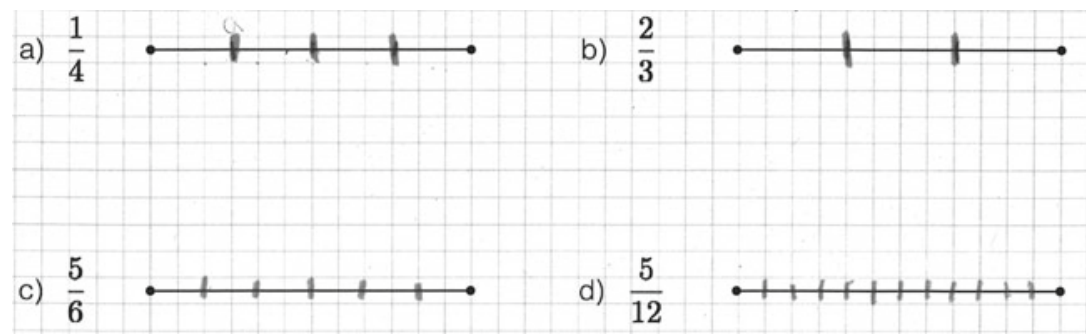

Abbildung 5.16 Lauras Bearbeitung von Aufgabe 4

Wie bereits am Anfang erklärt Laura: „Bei b) sind 2 Drittel, das verstehe ich nicht, wie viel das ist“ (8). Erneut reagiert Nike mit der Frage nach der Länge der ganzen Strecke, die sie dieses Mal jedoch selber beantwortet: „12 Kästchen, das sind 4, 4 Kästchen sind eine Einheit, also (zeichnet) so, so so" (10-11). Sie stellt fest, dass die Strecke erneut eine Länge von 12 Kästchen hat und wendet den ersten Teiloperator zum Teilen der Strecke an. Sie teilt also die Länge der Strecke und respektive die Anzahl durch 3 und sagt: ,4 Kästchen sind eine Einheit“ (10-11). Mit Einheit meint sie vermutlich Drittel - 4 Kästchen entsprechen einem Drittel der Strecke. In der Folge zeichnet sie nach je vier Kästchen der Strecke einen Trennstrich und teilt so die Strecke in drei Teile ein.

Laura wirft ein, dass sie sich „das nicht vorstellen“ (12) könne, womit sie vermutlich meint, dass sie sich nicht vorstellen kann, wie man diese Rechenhandlung auf die Darstellung der Strecke überträgt. Sie schaut auf auf die Zeichnung ihrer Partnerin. Nike erklärt ihr Vorgehen erneut: „So, so, so, das sind 12 Kästchen- 12 durch 3 sind 4, 4 Kästchen ein Strich, 4 Kästchen ein Strich, 4 Kästchen fertig“ (1315). Anhand Nikes Zeichnung erkennt Laura ihren (gedanklichen) Fehler: „Achso, du hast mehrere eingezeichnet. Ich hab nur eins eingezeichnet" (14-15). An dieser Äußerung wird deutlich, was Laura damit meinte, dass sich das nicht vorstellen könne. Sie stellt fest, dass ihre Partnerin ,mehrere eingezeichnet" hat, was bedeutet, dass Nike die ganze Strecke eingeteilt hat. Laura hingegen habe „,nur eins eingezeichnet“. Sie orientiert sich folglich an der Darstellung des ersten Bruchs $\frac{1}{4}$, für dessen Darstellung es genügt hat, ein Teilsegment in der Strecke einzuzeichnen und 
den Bruch damit bereits zu repräsentieren. In der Folge übernimmt sie das Vorgehen von Nike und beide Schülerinnen unterteilen die Strecke in Teilaufgabe c) in sechs und in Teilaufgabe d) in zwölf gleiche Teile.

Es fällt auf, dass Laura und Nike zwar alle Strecken korrekt einteilen, jedoch den zweiten Herstellungsschritt, das Färben des Anteils, auslassen (vgl. Abb. 5.16). Sie markieren keine Anteile, sondern sind mit ihren Ergebnissen zufrieden und gehen zur nächsten Aufgabe über. Für die Auslassung des zweiten Herstellungsoperators sind drei Erklärungen denkbar. Zum einen ist es möglich, dass die beiden Schülerinnen schlichtweg vergessen, den Anteil einzuzeichnen, ohne dass es einen besonderen Grund dafür gibt. Zum anderen wird jedoch deutlich, dass es für Nike und Laura und Nike nicht natürlich ist die Strecke einzuteilen und somit eine beträchtliche kognitive Belastung darstellt. Vor diesem Hintergrund ist es denkbar, dass bereits die Übertragung des ersten Herstellungsoperators und die Anpassung des Vorgehens an die neue Repräsentation eine besondere Herausforderung darstellen, sodass sie die Aufgabe danach als abgeschlossen ansehen. Drittens beschreibt Laura ihre Schwierigkeiten bei der Darstellung des Bruchs $\frac{2}{3}$ darin, dass sie sich das ,nicht vorstellen“ könne, während sie es sich zur Darstellung von $\frac{1}{4}$ vorstellen konnte. Ausgehend davon kann angenommen werden, dass sie den zweiten Teil der Herstellungshandlung - das Vervielfachen eines Teils - nicht auf die Darstellung in einer Strecke übertragen kann. Sie erklärt weiter, dass sie zuvor ,nur eins“ also nur einen Teil eingezeichnet habe, ihre Partnerin jedoch die ganze Strecke in gleiche Teile teilt. Sie könnte dieses Einteilen der ganzen Strecke bereits als Anwendung des zweiten Herstellungsschritts interpretieren.

Insgesamt können die Beobachtungen in Laura und Nikes Bearbeitung in folgenden Deutungshypothesen zusammengefasst werden:

- Der Repräsentationswechsel bzw. die Anwendung des Herstellungsverfahrens auf ein neues Repräsentationsobjekt bedarf der Anpassung des Verfahrens an die Eigenschatten der neuen Repräsentation. Im Falle des Wechsels von Flächendarstellungen, wie einem Kreis oder Rechteck auf die Darstellung einer Strecke, fällt es Laura schwer ein Maß bzw. die Größe zu identifizieren, auf die das Verfahren angewendet wird. Während zuvor Anteile zumeist als Fläche dargestellt wurden, ist die Größe für einen Anteil einer Strecke eine Länge. Da Laura dies nicht sofort erkennt, hat sie zunächst Schwierigkeiten bei der Anwendung des Verfahrens.

- Die hohe kognitive Belastung, die durch die Übertragung des Verfahrens und Anpassung an die Eigenschaften des neuen Repräsentationsobjekts aufgebaut wird, kann dazu führen, dass einzelne Lösungsschritte übergangen bzw. ausgelassen werden oder anders interpretiert werden als zuvor. In Laura und Nikes 
vorhergehenden Bearbeitungen war deutlich zu erkennen, dass sie großen Wert auf die Darstellung beider Herstellungsschritte gelegt haben, weshalb davon auszugehen ist, dass das fehlende Markieren des Anteils nicht auf ihr Unverständnis zurückzuführen ist. Vielmehr ist anzunehmen, dass sie diesen zweiten Schritt vergessen oder das Einteilen der ganzen Strecke als entsprechenden Handlungsschritt interpretieren.

\section{Luca \& Miguel - Darstellung nach Augenmaß}

In Luca und Miguels Bearbeitung des zweiten unvollständigen Beispiels war zu erkennen, dass sie auf symbolischer Ebene ohne Schwierigkeiten die richtigen Teiloperatoren zur Bruchherstellung ableiten können. In ihren ikonischen Darstellungen wurde jedoch deutlich, dass sie die Teiloperatoren auf enaktiver bzw. ikonischer Ebene nicht entsprechend deuten und ihre Interpretationen sich für verschiedene Repräsentationsobjekte unterscheiden (siehe 5.1.1). In der vorhergehenden Aufgabe 3 sollten die Lernenden verschiedene Brüche ikonisch darstellen. Die Zeichnungen von Luca und Miguel lassen erkennen, dass sie zur Darstellung der Brüche die Schritte zum Einteilen des Ganzen in gleiche Teile und Färben des Anteils durchführen, sondern die Anteile nach Augenmaß markieren, ohne eine Einteilung des Ganzen vorzunehmen (vgl. Abb. 5.17). Im Anschluss beginnen sie unmittelbar mit der Bearbeitung von Aufgabe 4.

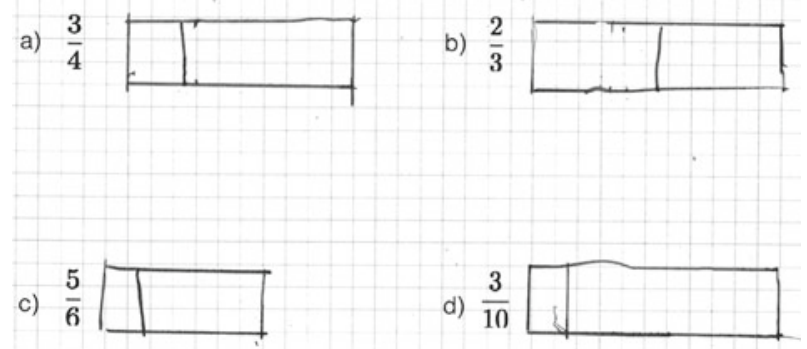

Abbildung 5.17 Lucas Bruchdarstellungen in Aufgabe 3

\section{Transkript B1A4 - Luca \& Miguel - Szene 1 - Aufgabe 4}

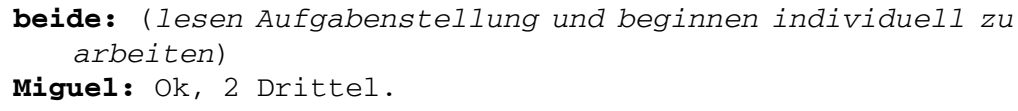



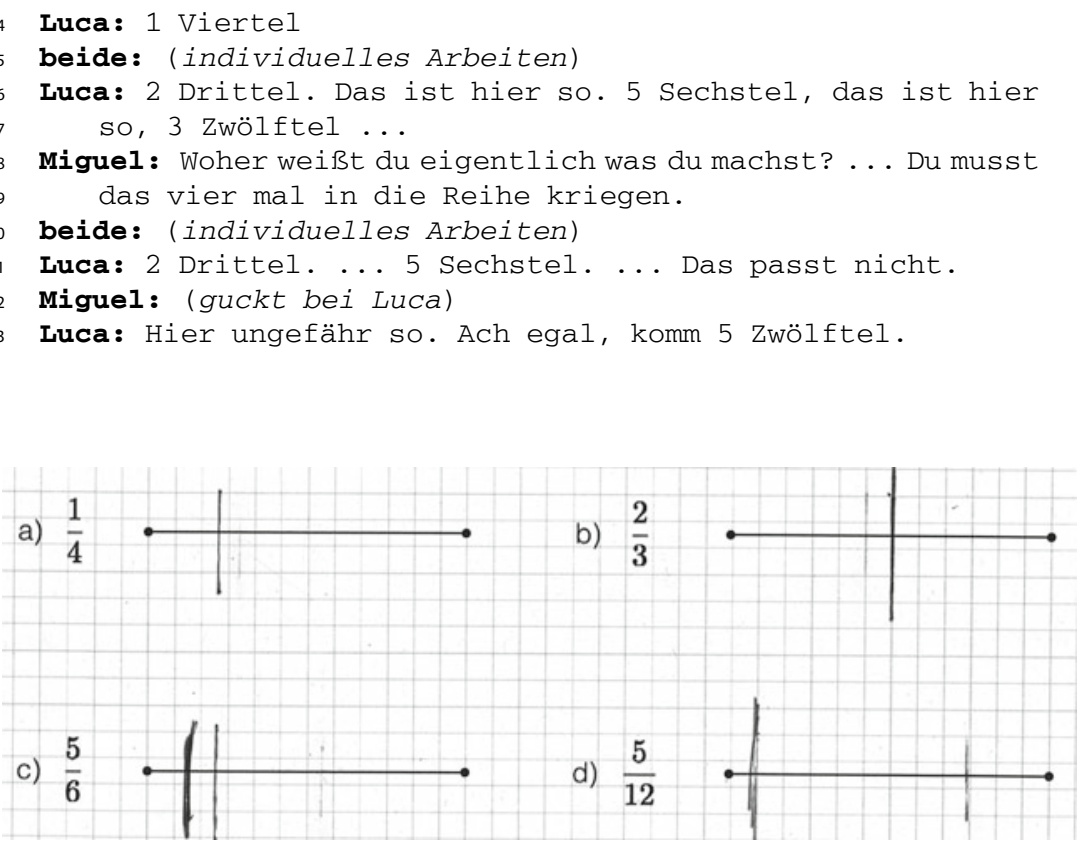

Abbildung 5.18 Lucas Bruchdarstellungen in Aufgabe 4

Luca und Miguel widmen sich der Aufgabe wie in den vorhergehenden Bearbeitungen zunächst ohne miteinander über die Aufgabe zu sprechen. Luca kommentiert: „Das ist hier so. 5 Sechstel, das ist hier so, 3 Zwölftel ...“ (6-7). Dabei lässt seine Beschreibung den Eindruck entstehen, dass er die Bruchteile nach Augenmaß an der Strecke abträgt. Die Genauigkeit seiner Markierungen scheint ihm dabei nicht sonderlich wichtig zu sein. In diesem Sinne interpretiert auch sein Partner Miguel seine Beschreibung, der ihn fragt: „Woher weißt du eigentlich was du machst?“ (8). Luca antwortet ihm auf diese Frage zunächst nicht, was die Deutung seines Vorgehens stützt, dass er die Länge der Bruchteile abschätzt. Miguel fügt mit Bezug auf den Bruch $\frac{1}{4}$ an, dass er ,das vier mal in die Reihe kriegen“ (8-9) müsse. Luca erwidert auf diese Bemerkung nichts, sondern überprüft und korrigiert seine Zeichnungen: „2 Drittel ... 5 Sechstel ... Das passt nicht“ (11). Er stellt scheinbar einen Fehler bei seiner Markierung des Bruchs $\frac{5}{6}$ in Teilaufgabe c) fest, den er umgehend korrigiert (vgl. Abb. 5.18). Erneut macht seine Äußerung nicht den Eindruck, die Bruchteile sonderlich genau einzeichnen zu wollen. Er kommentiert seine zeichnerische Kor- 
rektur mit den Worten „Hier ungefähr so. Ach egal, komm 5 Zwölftel“ (13). Miguel übernimmt Lucas Darstellungen ohne diese weiter zu hinterfragen.

Die eingehende Betrachtung von Lucas Darstellungen stützt die Annahme, dass er, wie sein Partner Miguel festgestellt hat, zunächst durch Abschätzen und nach Augenmaß die Bruchteile an der Strecke abträgt. Er orientiert sich dabei nicht unbedingt an dem den Streckenabbildungen unterliegenden Kästchenmuster, wie in seiner Darstellung in Teilaufgabe zu erkennen ist. Auf den Hinweis von Miguel, dass der Streckenabschnitt mit der Länge $\frac{1}{4}$ vier mal in die ganze Strecke passen müsse, nimmt er diese Eigenschaft des Anteils als Anlass seine Zeichnungen zu überprüfen und dementsprechend zu korrigieren. Dabei orientiert er sich bei den Brüchen $\frac{5}{6}$ und $\frac{5}{12}$ ausschließlich am Nenner der Brüche und markiert in etwa ein Sechstel und ein Zwölftel der Strecke durch einen Trennstrich. Er überträgt somit das Vorgehen aus Teilaufgabe a) auf die nachfolgenden Bruchdarstellungen. Die Ausnahme zu diesem Vorgehen ist der Bruch $\frac{2}{3}$, für den er die Strecke in zwei Teile teilt, was so erklärt werden kann, dass er sich hier am Zähler des Bruchs orientiert und versucht ein Segment der Strecke zu kennzeichnen, das zweimal in die ganze Strecke hineinpasst.

Insgesamt wird deutlich, dass Luca und Miguel nicht das Bruchherstellungsverfahren übertragen um Bruchteile an einer Strecke zu markieren. Stattdessen orientieren sich an der Anteilvorstellung zu Stammbrüchen, dass $n \cdot \frac{1}{n}=1 \mathrm{bzw}$. ein Ganzes ist. Dabei werden Unsicherheiten bezüglich der Bedeutung von Zähler und Nenner erkennbar. Es kann angenommen werden, dass die fehlende Anwendung des Bruchherstellungsverfahrens auf die Darstellung an einer Strecke darauf zurückzuführen ist, dass sie fehlerhafte Herstellungshandlungen mit den Teiloperatoren zur Bruchherstellung verbinden, wie in ihrer Bearbeitung des zweiten unvollständigen Beispiels zu erkennen war. Die Beobachtungen in dieser Aufgabe stärken weiterhin die Deutung, dass Luca und Miguel die anschauliche Ebene und die rechnerische Ebene des Bruchherstellungsverfahrens nicht miteinander in Beziehung setzen. Aus diesem Grund haben sie Schwierigkeiten bei der ikonischen Darstellung von Bruchteilen und kein Handlungsschema verfügbar, an dem sie sich orientieren können.

Die Bearbeitung von Luca und Miguel kann in den folgenden Deutungshypothesen zusammengefasst werden:

- Luca und Miguel übertragen das Bruchherstellungsverfahren nicht auf die Darstellung von Bruchteilen an einer Strecke. Aus Ermangelung eines Herstellungsschemas versucht Luca zunächst die Anteile an der Strecke nach Augenmaß abzuschätzen.

- Im Weiteren orientieren sich sich an der Anteilvorstellung zu Stammbrüchen, dass $\frac{1}{n} n$-mal in die ganze Strecke passen muss. Mit Ausnahme eines Bruchs, bei 
dem sie sich am Zähler und nicht am Nenner des Bruchs orientieren, übertragen sie dieses Vorgehen auf die nachfolgenden Bruchdarstellungen und markieren entsprechend jeweils nur einen Teil des Bruchteils.

- Da bereits in ihren vorhergehenden Bearbeitungen eine Entkopplung von symbolischer und anschaulicher Ebene angenommen wurde, wird diese Annahme weiter gestützt. Luca und Miguel verbinden die Teilschritte der Bruchherstellung nicht mit entsprechenden anschaulichen Vorstellungen und können das Herstellungsschema in der Folge nicht übertragen und anwenden.

\section{Vergleich der Bearbeitungen zur Bruchdarstellung an einer Strecke}

Auf normativer Ebene stellt sich der Transfer des Bruchherstellungsverfahrens auf die Darstellung von Bruchteilen $\frac{m}{n}$ an einer Strecke in Form von zwei Transferschritten dar:

- Anwenden des Teiloperators: $n$ zum Einteilen der Strecke in $n$ gleich lange Streckenabschnitte und

- Anwenden des Teiloperators $\cdot m$ zum Markieren von $m$ Streckenabschnitten.

Die Beobachtungen der Analysen umfassen daher im Wesentlichen Aspekte der Übertragung des Operatorschemas sowie der Anpassung des Herstellungsverfahrens an die neue Repräsentation.

Übertragung des Operatorschemas: Mit Ausnahme der Bearbeitung von Luca und Miguel sind in allen Analysen deutlich die Übertragung von Aspekten des Operatorschemas in Form des Bruchherstellungsverfahrens zu erkennen. Bennet und Julius übertragen dieses Verfahren quasi spontan und ohne Zögern. In Bezug auf die Anwendung sind jedoch Unterschiede im Vorgehen von Bennet und Julius zu erkennen. Julius orientiert sich strikt an dem Handlungsschema Aufteilen und Markieren. Er bestimmt die Länge der ganzen Strecke in der Einheit 1 Kästchen, teilt die Länge rechnerisch in gleiche Teile und markiert die entsprechende Anzahl der Teile. Im Gegensatz zu Julius ist in den schriftlichen Dokumenten zu erkennen, dass Bennet die Länge der Teilsegmente nicht rechnerisch bestimmt, sondern „,so kurz über'n Daumen“ die Strecke unterteilt. Dieses Vorgehen lässt vermuten, dass er sich im Zuge einer Abstraktion beginnt von der schematischen Ausführung des Verfahrens zu lösen, indem er zwar das grundlegende Handlungsschema anwendet, aber scheinbar bereits ein mentales Bild vor Augen hat, an dem er sich orientiert, und keine Notwendigkeit sieht, die Länge der einzelnen Streckenabschnitte rechnerisch exakt zu bestimmen. 
Genau wie Julius wendet auch Aliya ohne Schwierigkeiten das Operatorschema zur Berechnung der Segmentlänge und zum Markieren des Anteils an. Anders verhält es sich bei ihrer Partnerin Aliya, die zur Darstellung der Anteile an einer Strecke vorwiegend eine statisch-abbildhafte Vorstellung eines Bruchs als Anteil einer diskreten Menge aktiviert. Anders als ihre Partnerin stellt sie keinen Bezug zur Herstellung des Bruchs her, sondern deutet den Nenner des Bruchs entsprechend der Fehlvorstellung $\frac{1}{n}$ bezeichnet eine Menge von $n$ Objekten als Anzahl der zu markierenden Kästchen, ohne dabei einen Bezug zur ganzen Strecke herzustellen. Im Gegensatz zu ihrer Partnerin, die durch ihre dynamische Sichtweise die vorgegebenen Brüche mit ihrer Herstellungshandlung verknüpft, betrachtet Aisha Brüche statisch als Anzahl von Teilen. Sie formuliert zwar, dass $\frac{1}{4}$, ,eins von vier Stücken“ bedeutet, betrachtet dann aber nur den Nenner, was auf eine Verhaftung ihres Denkens an die natürlichen Zahlen hindeutet.

Ein ähnliches Erklärungsmodell kommt auch in der Bearbeitung von Luca und Miguel zum Tragen. Die Bearbeitungen der beiden Schüler deuteten bereits zuvor eine Entkopplung der symbolischen und anschaulichen Ebene hin, bei der sie die symbolischen Herstellungsoperatoren mit fehlerhaften Herstellungshandlungen übersetzt haben. In ihrer Bearbeitung kommt das Operatorschema nicht zur Anwendung. Stattdessen betrachten sie ausschließlich die Nenner $n$ der vorgegebenen Brüche $\frac{m}{n}$ und markieren jeweils ein Streckensegment der Länge $\frac{1}{n}$. Das ihrer Bearbeitung zugrunde liegende Erklärungsmodell ist, dass $\frac{1}{n} n$-mal in die ganze Strecke passen muss. Auch hier ist eine Orientierung am Umgang mit natürlichen Zahlen anzunehmen, die sich in der Deutung der Brüche auf Grundlage der Anteilvorstellung von Stammbrüchen äußert.

In der Bearbeitung von Laura und Nike ist die teilweise Anwendung des Operatorschemas zu erkennen. Sie bestimmen die Länge der Strecke in der Größeneinheit 1 Kästchen und berechnen die Länge der Streckensegmente. Jedoch tragen sie im Anschluss daran nicht die vorgegebenen Anteile ein, sondern beschränken sich auf die Einteilung der Strecke in je $n$ gleiche Teile. Die Äußerungen der beiden Schülerinnen deuten auf Schwierigkeiten bei der Übertragung des zweiten Teiloperators auf die neue Repräsentation hin. Während sie bei der Darstellung von $\frac{1}{4}$ keine Schwierigkeiten formulieren, bemerkt Laura beim Einzeichnen des Bruchs $\frac{2}{3}$, dass sie sich das nicht vorstellen könne. Es ist anzunehmen, dass sie sich dabei auf die Anwendung des zweiten Teiloperators zum Vervielfachen eines Teils bezieht.

Die Anwendung des Bruchherstellungsverfahrens und respektive die Anwendung des Operatorschemas zur Darstellung von Brüchen an einer Strecke ist insbesondere von der Anwendung auf eine Strecke als neues Repräsentationsobjekt und der Anpassung des Verfahrens an die Eigenschaften einer Strecke geprägt. 
Anpassung des Herstellungsverfahrens an die neue Repräsentation: Beim Vergleich der dargestellten Bearbeitungsprozesse wird erkennbar, dass die Anwendung des Bruchherstellungsverfahrens auf eine neue Repräsentation, wie in diesem Fall die Darstellung an einer Strecke, die Koordination von wenigstens drei subjektiven Erfahrungsbereichen bzw. Grundvorstellungen erfordert:

- Der Vorstellung von Brüchen als Anteil: $\frac{m}{n}$ bedeutet $m$ von $n$ Teilen,

- die Vorstellung der Bruchherstellungshandlung: $\frac{m}{n}$ bedeutet, das Ganze wird in $n$ Teile geteilt, von denen $m$ markiert werden sowie

- dem subjektiven Erfahrungsbereich „Operieren mit einer Strecke“.

Die Argumentationen von Aliya, Bennet und Julius spiegeln diese Koordination wieder. Sie erkennen die vorgegebenen Brüche als Anteile, bringen diese Anteile mit ihrer Herstellungshandlung in Verbindung und erkennen, wie sie diese Herstellungshandlung an einer Strecke durchführen können. In Julius Argumentation ist weiterhin eine Orientierung an Handlungen mit konkreten Gegenständen erkennbar. Im Gegensatz zur Bearbeitung der unvollständigen Beispiele zuvor, in der die Orientierung am Umgang mit gegenständlichen Handlungsobjekten zur Wahl der falschen Rechenoperation führte, unterstützt sie in diesem Fall den Transfer des Herstellungsverfahrens auf die Repräsentation an einer Strecke.

Im Gegensatz dazu ist Lauras Argumentationen zu entnehmen, dass sie zunächst einmal Schwierigkeiten bei der Aktivierung des subjektiven Erfahrungsbereichs „Operieren mit einer Strecke“ hat. Sie hat zunächst Probleme zu identifizieren, welches Maß bzw. welche Größeneinheit beim Umgang mit einer Strecke zu verwenden ist. Erst als ihre Partnerin ihr erklärt, dass sie die Länge der Strecke ermitteln müsse, findet sie einen Zugang zur Darstellungshandlung. In der Folge gelingt es ihr korrekt $\frac{1}{4}$ der Strecke zu kennzeichnen. Eine weitere Schwierigkeit in ihrem Bearbeitungsprozess stellt die Übertragung des zweiten Herstellungsoperators auf die neue Repräsentation dar. Bei der Darstellung des Bruchs $\frac{2}{3}$ erklärt sie, dass sie das nicht verstehe, wobei sie sich sehr wahrscheinlich auf die Übersetzung des Zählers bezieht. Sie erkennt im weiteren, dass ihre Partnerin Nike, anders als Laura, bei der Darstellung von $\frac{1}{4}$ nicht nur den Bruchteil kenntlich gemacht hat, sondern die ganze Strecke in gleich große Teile eingeteilt hat, was sie im weiteren als Anwendung des zweiten Herstellungsoperators interpretiert. Entsprechend kann im Fall von Laura und Nike die Schwierigkeit bei der Bearbeitung auf die Koordination der Bruchherstellungshandlung und des Operierens mit einer Strecke zurückgeführt werden. Es gelingt ihnen nur für den ersten Herstellungsschritt eine Analogie zwischen der Herstellungshandlung an einer Flächenrepräsentation (Kreis oder Rechteck) und der linearen Darstellung an einer Strecke herzustellen. 
Auch die beobachteten Schwierigkeiten in den Bearbeitungsprozessen von Aisha, Luca und Miguel lassen sich durch ein unzureichende Koordination der oben genannten Vorstellungen bzw. Erfahrungsbereiche beschreiben. Die Bearbeitungsprozesse der drei Schülerinnen und Schüler haben gemeinsam, dass zwar die Anteilvorstellung und auch den Erfahrungsbereich des Operierens mit einer Strecke aktivieren. Es sind jedoch keine Anzeichen für die Aktivierung der Operatorvorstellung bzw. des Bruchherstellungsverfahrens zu erkennen und sie interpretieren die vorgegebenen Brüche vor allem als statische Entitäten. Aisha deutet den Bruch $\frac{1}{4}$ als „eins von vier Stücken“ und Luca und Miguel deuten den Bruch $\frac{1}{4}$ so, dass das entsprechende Streckensegment vier mal in die ganze Strecke passen muss. Insgesamt kann angenommen werden, dass die Schwierigkeiten von Aisha, Luca und Miguel auf eine noch nicht erfolgte oder fehlerhafte Verknüpfung der Anteilund Operatorvorstellung zurückzuführen ist. In Luca und Miguels Bearbeitung der unvollständigen Beispiele wurde deutlich, dass sie die Herstellungsoperatoren mit fehlerhaften Handlungen auf ikonischer Ebene übersetzen. Aufgrund dessen haben sie bereits grundlegende Schwierigkeiten bei der Darstellung von Brüchen in einem Kreis oder Rechteck. In der Folge mangelt es ihnen an einem konkreten Handlungsschema, an dem sie sich orientieren können und dass sie an die Eigenschaften einer Strecke anpassen können.

\subsubsection{Vergleich der Bearbeitungsprozesse zu den beiden Transferaufgaben}

Die hier dargestellten Analysen der Bearbeitungsprozesse der Schülerinnen und Schüler können in der Charakterisierung von drei zentralen Transferprozessen zusammengefasst werden:

(i) Der Verknüpfung der Herstellungshandlungen auf symbolischer und anschaulicher Ebene,

(ii) die Verknüpfung von Brüchen als Anteile eines Ganzen mit ihrer Herstellungshandlung und

(iii) die Aktivierung und Koordination verschiedener Erfahrungsbereiche zur Anpassung des anschaulichen Herstellungsverfahrens auf die Eigenschaften der neuen Repräsentation.

Für die Anwendung des Operatorschemas zur Erklärung der Herstellung von Brüchen stand insbesondere die Verknüpfung der Herstellungshandlung auf symbolischer und anschaulicher Repräsentationsebene im Zentrum des Transfers. In den 
Analysen wurde aufgezeigt, dass es für die Übertragung des Herstellungsverfahrens von besonderer Bedeutung ist, die Teiloperatoren der Herstellung mit adäquaten Herstellungshandlungen auf anschaulicher Ebene in Beziehung zu setzen. Diesbezüglich konnte beobachtet werden, dass die Formulierung des „Nehmens von $m$ Teilen“ sinngemäß mit einem Wegnehmen und entsprechend einer Subtraktion von $m$ Teilen übersetzt wird. Zudem war zu beobachten, dass mit dem Divisionsoperator ein Wegnehmen von Teilen und mit dem Multiplikationsoperator ein Hinzufügen von Teilen anstelle des Vervielfachen eines Teils verbunden wurde. Fehlerhafte Übersetzungen dieser Art hatten vor allem die Wahl der falschen Rechenoperationen sowie eine Entkopplung der symbolischen und anschaulichen Ebene der Bruchherstellung zur Folge.

In den Bearbeitungen zur Darstellung von Brüchen an einer Strecke konnte in diesem Zusammenhang festgestellt werden, dass eine unzureichende Verknüpfung von Brüchen mit ihrer Herstellungshandlung bzw. des Bruchherstellungsverfahrens zu einer statischen Sichtweise auf Brüche und der Dominanz der Anteilvorstellung führt. Die Schülerinnen und Schüler, die die vorgegebenen Anteile nicht mit ihrer Herstellung in Verbindung gebracht haben, hatten Schwierigkeiten bei der Darstellung an der Strecke und somit beim Repräsentationstransfer, was möglicherweise zur Verfestigung der Entkopplung der symbolischen und anschaulichen Ebene beiträgt. In Bezug auf die Anwendung der Herstellungshandlung auf die Darstellung von Brüchen in unterschiedlichen ikonischen Repräsentationen konnte in diesem Zusammenhang beobachtet werden, dass die Herstellungshandlungen bei der Anwendung auf unterschiedliche Repräsentationsobjekte unterschiedlich gedeutet werden, was der intendierten Förderung der Abstraktion des Verfahrens und Loslösung von spezifischen Repräsentationsobjekten entgegen wirken kann.

Bei der Übertragung des Herstellungsverfahrens auf die Darstellung von Brüchen an unterschiedlichen ikonischen Repräsentationen wurde deutlich, dass die Vorstellung von Brüchen als Anteile, die Herstellungshandlung von Brüchen und die Eigenschaften des betreffenden Repräsentationsobjekt als zunächst voneinander getrennte subjektive Erfahrungsbereiche betrachtet werden können. In den Bearbeitungen konnte beobachtet werden, dass eine fehlende Koordination bzw. Verknüpfung dieser Erfahrungsbereiche zu fehlerhaften Darstellungen und Verständnisschwierigkeiten führen kann. In den analysierten Bearbeitungen wurden Fälle dargestellt, in denen eine unzureichende Verknüpfung von Anteilen mit ihrer Herstellungshandlung zu Schwierigkeiten oder fehlerhaften Darstellungen führte. Zum anderen wurde ein Fall dargestellt, in dem eine Schülerin zunächst keinen Größenbereich zur Darstellung an einer Strecke identifiziert hat. In den Fällen, in denen die Schülerinnen und Schüler erfolgreich die Herstellungshandlung zur Darstellung von Brüchen an einer Strecke übertragen haben, konnte hingegen festgestellt werden, 
dass sie die Brüche mit ihrer entsprechenden Herstellungshandlung in Beziehung gesetzt und diese an die Eigenschaften bzw. den Größenbereich des neuen Repräsentationsobjekt angepasst haben.

\subsection{Anteile von beliebigen Größen}

In diesem Abschnitt werden die Analysen der Daten aus der zweiten Datenerhebung dargestellt. Dazu werden zunächst zum Überblick die eingesetzten Lernmaterialien beschrieben. Diese umfassen wie zuvor ein interaktives animiertes Lösungsbeispiel, zwei unvollständige Beispiele sowie ausgewählte Transferaufgaben. Die Beschreibung der Materialien erfolgt auch sachanalytischer Ebene mit Bezug zu den enthaltenen Grundvorstellungen. Die normativen Analysen bilden den Ausgangspunkt für die Beschreibung der in den Transferaufgaben erforderten Transferprozesse.

In der Zeit zwischen der ersten und zweiten Datenerhebung haben die Schülerinnen im Klassenunterricht mit dem Arbeitsbuch weitergearbeitet. In zwölf Unterrichtsstunden haben sie dabei den Inhalt der ersten Datenerhebung weiter vertieft und zudem folgende Inhalte behandelt:

- Brüche als Teile eines Ganzen - Unechte Brüche und gemischte Schreibweise

- Brüche als Teile eines Ganzen - Brüche als Maßzahlen in Größenangaben

- Brüche als Teile mehrerer Ganzer

Lösungsbeispiel: Die Eingangsphase der Unterrichtsstunde beginnt mit dem Lesen eines digitalen animierten Lösungsbeispiels in zwei Teilen. Der Kontext ist für beide Teile ein gerechtes Aufteilen eines Geldgewinns von $21000 €$ auf die zwei Familien Meyer und Familie Stein. Familie Meyer besteht dabei aus drei, Familie Stein aus vier Personen.

Im ersten Teil des Lösungsbeispiels (vgl. Abbildung 5.19) werden die Anteile am Gewinn für jede Familie bestimmt. Dazu wird $21000 €$ als Ganzes interpretiert und durch einen Kreis repräsentiert. Da der Anteil entsprechend der Anzahl an Personen jeder Familie bestimmt wird, wird das Ganze auf die Anzahl aller Personen der beiden Familien in sieben gleich große Teile aufgeteilt. Ein Teil ist $\frac{1}{7}$ des Ganzen und wird durch ein Stück des Kreises repräsentiert. Jede Person erhält ein Teil des Ganzen und somit ein Stück des Kreises.

Familie Meyer besteht aus drei Personen und erhält entsprechend drei Teile, also $\frac{3}{7}$ des Ganzen. In einer Animation werden drei Teile des Kreises in Richtung der Familie verschoben. Familie Stein besteht aus vier Personen und erhält somit vier 
Teile des Ganzen. Die vier Teile der Familienmitglieder bewegen sich zusammen zu einem Anteil, der $\frac{4}{7}$ beträgt.

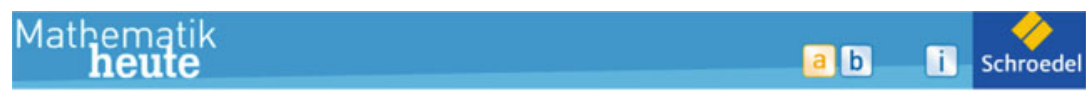

Familie Meyer (3 Personen) und Familie Stein (4 Personen) spielen gemeinsam Lotto. Sie haben vereinbart, einen Gewinn auf alle 7 Familienmitglieder gleichmäßig zu verteilen. Am letzten Spieltag haben sie gemeinsam $21000 €$ gewonnen.

a) Welchen Anteil am Gewinn erhält jede Familie?

\section{Lösung:}

Das Ganze ist der Gewinn, also $21000 €$.

Er muss in 7 gleich große Teile zerlegt werden.

Jeder Teil ist $\frac{1}{7}$ des Gewinns.

3 dieser Teile erhält Familie Meyer, das sind $\frac{3}{7}$ des Gewinns.

4 dieser Teile erhălt Familie Stein, das sind $\frac{4}{7}$ des Gewinns.

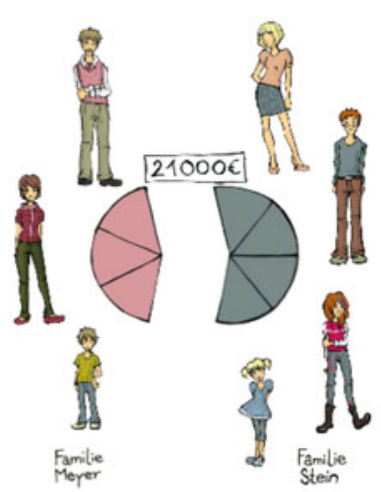

\section{Mathematik} heute

Familie Meyer (3 Personen) und Familie Stein (4 Personen) spielen gemeinsam Lotto. Sie haben vereinbart, einen Gewinn auf alle 7 Familienmitglieder gleichmäßig zu verteilen. Am letzten Spieltag haben sie gemeinsam $21000 €$ gewonnen.

b) Wie viel Euro erhält jede Familie?

\section{Lösung:}

Familie Meyer erhält $\frac{3}{7}$ von $21000 €$ :

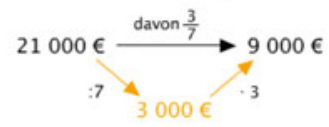

$21000 €: 7=3000 €$ $3000 € \cdot 3=9000 €$

Ergebnis: Familie Meyer erhält $9000 €$.

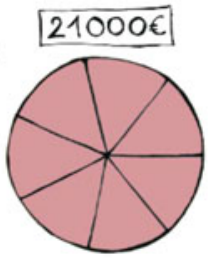

$€: 7=3000 €$

$3000 € \cdot 4=12000 €$

Ergebnis: Familie Stein erhält $12000 €$.

Abbildung 5.19 Endzustand der Teile a) und b) des animierten Lösungsbeispiels 
Im zweiten Teil des Beispiels werden die Anteile am Gewinn für beide Familien anhand eines Pfeildiagramms berechnet (siehe Abbildung 5.19).

Familie Meyer erhält $\frac{3}{7}$ und Familie Stein $\frac{4}{7}$ des $21000 €$ Gewinns. Zur Berechnung wird der Gewinn zunächst durch 7 geteilt $-\frac{1}{7}$ von $21000 €$ sind $3000 €-$ und für Familie Meyer schließlich mit 3 und für Familie Stein mit 4 multipliziert. Somit erhält Familie Meyer $9000 €$ und Familie Stein $12000 €$. Die Berechnung der Anteile nacheinander für jede Familie in einem Pfeildiagramm dargestellt. Die Pfeildiagramme bauen sich als Animation Schritt für Schritt auf.

Unter der Darstellung der Rechenoperationen in den Pfeildiagrammen, wird die Rechnung zusätzlich in schrittweiser Form dargestellt und jeweils mit einem Ergebnissatz ergänzt.

In den Arbeitsheften der Lernenden werden die statisch abgebildeten Teile des Lösungsbeispiels mit folgenden fokussierenden Fragestellungen ergänzt:

Lösungsbeispiel Teil a): Erkläre, warum Familie Meyer $\frac{3}{7}$ und Familie Stein $\frac{4}{7}$ erhält.

Lösungsbeispiel Teil b): Was bedeutet $\frac{3}{7}$ von $21000 €$ und wie kann man das berechnen?

Die erste fokussierende Fragestellung bezieht sich auf das Nachvollziehen der Aufteilung des Gewinns auf die beiden Familien. Im Vordergrund steht hierbei die Argumentation, dass die beiden Familien unterschiedliche Anzahlen an Familienmitgliedern haben. Die zweite fokussierende Frage bezieht sich auf das Verfahren der Anteilberechnung. Für die Beantwortung dieser Frage wird erwartet, dass die Lernenden beschreiben, dass der Gewinn durch die Division :7 in sieben gleiche Teile zu je $3000 €$ aufgeteilt wird. $\frac{3}{7}$ entsprechen drei dieser sieben Teile und man erhält den Anteil durch Vervielfachen eines Teils durch die Multiplikation ·3.

Ausgewählte Transferaufgaben: Im Anschluss an die interaktiven animierten Lösungsbeispiele folgt eine Serie von Übungs- und Transferaufgaben (siehe Tabelle 5.2). Detaillierte Aufgabenbeschreibungen und Erläuterungen der Transferprozesse erfolgen an den entsprechenden Stellen in diesem Abschnitt. Der Haupttransfer in dieser Sitzung ist der Übergang von konkreten Gegenständen zu beliebigen Größen. In der Unterrichtseinheit wurden zunächst Anteile von konkreten Gegenständen, z. B. einer Pizza oder eines Kuchens, gebildet. Im Gegensatz dazu sind die Ganzen in den nachfolgenden Aufgaben keine gegenständlichen Ganzen, sondern (abstrakte) Größen, wie z. B. ein Geldbeträge, Längen oder Mengen von Objekten. Dieser Transfer der Anteilbildung von konkreten Gegenständen auf abstrakte Größen spielt in allen Bearbeitungen eine wichtige Rolle, auch wenn er nicht zwingend in den Aufgaben enthalten ist. 
Tabelle 5.2 Transferaufgaben - Anteile von beliebigen Größen

\begin{tabular}{l|l|l}
\hline & Aufgabe & Zentrale Transferprozesse \\
\hline $2.1 \& 2.2$ & $\begin{array}{l}\text { Unvollständige } \\
\text { Lösungsbeispiele }\end{array}$ & $\begin{array}{l}\text { Übertragung des Verfahrens zur } \\
\text { Anteilbestimmung und -Berechnung aus } \\
\text { dem Lösungsbeispiel auf eine analoge } \\
\text { und strukturähnliche Sachsituationen }\end{array}$ \\
\hline 2.9 & Umkehrung der Anteilbildung & $\begin{array}{l}\text { Umkehrung der Anteilbildung zur } \\
\text { Bestimmung zur Bestimmung des } \\
\text { Ganzen }\end{array}$ \\
\hline
\end{tabular}

\subsubsection{Unvollständige Beispiele}

Erstes unvollständiges Beispiel: Das erste unvollständige Beispiel ist strukturgleich mit dem vorhergehenden Lösungsbeispiel (siehe Abb. 5.20). Ein Geldgewinn von $900 €$ soll auf zwei Familien, die Familie Schmidt und die Familie Tenner, aufgeteilt werden, wobei Familie Schmidt aus zwei Personen und Familie Tenner aus vier Personen besteht.

Analog zum ersten Teil des Lösungsbeispiels soll zunächst der Anteil bestimmt werden, den jede Familie vom Gewinn erhält. Als Hilfestellung wird im Aufgabentext bereits vorweggenommen, dass das Ganze entsprechend der Anzahl der Personen beider Familien in sechs gleiche Teile geteilt wird, die auf die beiden Familien verteilt werden. Jeder Teil ist ein Sechstel des Gewinns. Da Familie Schmidt zwei Familienmitglieder hat erhält sie zwei Teile, also $\frac{2}{6}$ vom Ganzen. Familie Tenner erhält mir vier Familienmitgliedern $\frac{4}{6}$ vom Ganzen. Zur Veranschaulichung der Aufteilung des Ganzen sollen die Lernenden in einer vorgegebenen Kreisrepräsentation die Anteile einzeichnen.

Die jeweiligen Lösungsschritte sind als Bearbeitungshilfen in Sprechblasen notiert. Sie enthalten die Hinweise: „Wie viele Sechstel erhält jede Familie?“ und „Teile passend ein und färbe die Anteil der Familien.“

Im zweiten Teil des unvollständigen Beispiels sollen die den Anteilen entsprechenden Geldbeträge für jede Familie berechnet werden. Analog zur Struktur im Lösungsbeispiel sind für diese Rechnungen zwei unvollständige Pfeilschemata dargestellt, in denen die entsprechenden Rechenoperationen und Ergebnisse eingetragen werden sollen. Zum Abschluss sollen die Schülerinnen und Schüler analog zum Lösungsbeispiel einen Antwortsatz formulieren, dessen Anfang bereits vorgegeben ist. In zwei Sprechblasen werden Hinweise zur Bearbeitung gegeben: Mit Verweis auf die Pfeildarstellungen enthalten sie den Auftrag „Ergänze die Pfeilbilder“ und 


\section{Aufgabe 1}

Familie Schmidt (2 Personen) und Familie Tenner (4 Personen) haben bei einer Verlosung $900 €$ gewonnen. Das Geld soll auf alle Familienmitglieder gleichmäßig verteilt werden.

a) Welchen Anteil am Geld erhält jede Familie?

b) Wie viel Euro bekommt jede Familie?

\section{Lösung:}

a) 900 Euro sind das ganze gewonnene Geld.

Es wird in 6 gleich große Teile zerlegt.

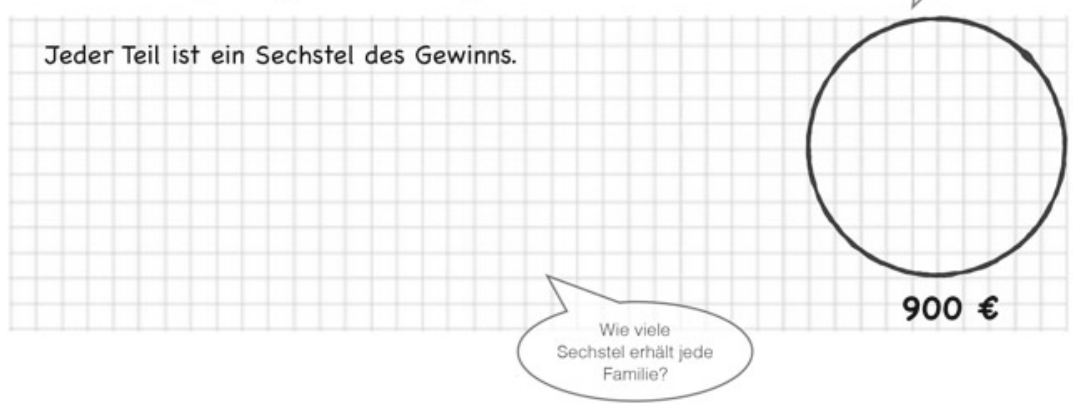

b) Familie Schmidt:

Familie Tenner:

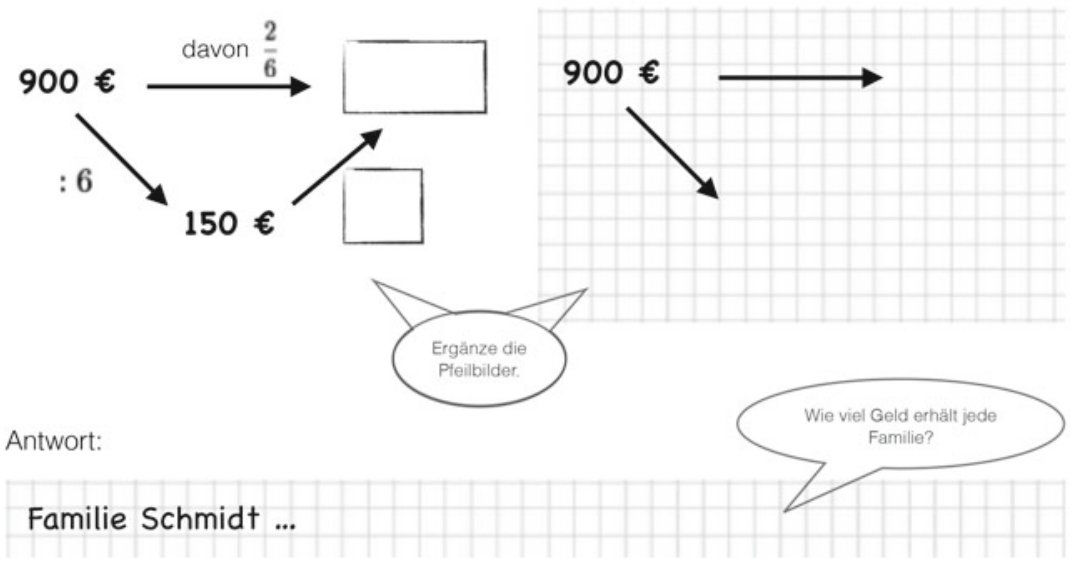

Abbildung 5.20 Unvollständiges Beispiel - Aufgabe 2.1 
mit Verweis auf den Lösungssatz wird die Frage formuliert „Wie viel Geld erhält jede Familie?“.

Transferprozesse im ersten unvollständigen Beispiel: Das erste unvollständige Beispiel ist sehr nah am Lösungsbeispiel gestaltet. Das bedeutet, dass der Sachkontext und die beinhalteten Größen dieselben sind. Der zentrale Transferprozess ist in diesem Zusammenhang die Übertragung der einzelnen Lösungsschritte, dem Bestimmen der Anteile für die beiden Familien und die Berechnung der entsprechenden Geldbeträge. Die einzigen Unterschiede zum Lösungsbeispiel betreffen die Personenzahl der beiden Familien und den aufzuteilenden Geldbetrag: Der Gewinn beträgt nicht $21000 €$, sondern $900 €$ und die Familien bestehen aus zwei und vier Personen, weshalb der Gewinn nicht in sieben, sondern in sechs gleiche Teile geteilt wird und die entsprechenden Anteile $\frac{2}{6}$ von $900 €$ sowie $\frac{4}{6}$ von $900 €$ sind.

Zweites unvollständiges Beispiel: Im zweiten unvollständigen Beispiel soll erneut ein Geldbetrag gerecht auf zwei Parteien aufgeteilt werden. Im Gegensatz zum Lösungsbeispiel und dem ersten unvollständigen Beispiel wurde der Sachkontext leicht verändert. Zwei Jugendliche, Lisa und Jan, haben in einem Jugendclub gearbeitet und gemeinsam $84 €$ als Lohn für ihre Arbeit bekommen, den sie gerecht untereinander aufteilen wollen. Da Lisa mit sieben Stunden mehr gearbeitet hat als Jan, der fünf Stunden gearbeitet hat, muss der Gewinn für eine gerechte Teilung entsprechend der geleisteten Arbeitsstunden aufgeteilt werden (Abb. 5.21).

Zur Strukturierung des Lösungsweges ist die Aufgabenstellung zweigeteilt. Im ersten Schritt sollen die jeweiligen Anteile für Lisa und Jan bestimmt werden. Da die beiden Jugendlichen zusammen $7+5=12$ Stunden gearbeitet haben, muss der Lohn in zwölf gleiche Teile aufgeteilt werden. Da Lisa sieben der insgesamt zwölf Stunden gearbeitet hat, bekommt sie sieben Teile, also $\frac{7}{12}$ vom Ganzen. Jan hat fünf der zwölf Stunden gearbeitet und bekommt somit $\frac{5}{12}$ des Ganzen. Im Arbeitsheft ist anders als im unvollständigen Beispiel zuvor kein Lösungsansatz vorgezeichnet. Es ist lediglich eine Fläche mit Kästchenmuster vorgegeben, in der die Lernenden ihren Lösungsweg notieren sollen. Zur Veranschaulichung findet sich auf der rechten Seite die Abbildung eines Kreises, der mit $84 €$ unterschrieben ist, in den die Schülerinnen und Schüler die Anteile am Gewinn einzeichnen können. In einer Sprechblase ist der Hinweis notiert: „Gib den Anteil an, den jeder bekommt. Du kannst die Anteile auch einzeichnen“.

Im zweiten Aufgabenteil sollen die Anteile berechnet und die Frage beantwortet werden, wie viel Euro Lisa und Jan jeweils bekommen. Dazu sind zwei getrennte und mit den Namen von Lisa und Jan überschriebene Bearbeitungsflächen vorgegeben. In einer Sprechblase wird der Hinweis gegeben „Hier kannst du rechnen oder ein 


\section{AufGabe 2}

Lisa und Jan haben in den Ferien in einem Jugendclub gearbeitet. Sie bekommen $84 €$. Lisa hat 7 Stunden und Jan hat 5 Stunden gearbeitet.

a) Welchen Anteil am Geld bekommt Lisa, welchen Anteil bekommt Jan?

b) Wie viel Euro bekommt Lisa, und wie viel Jan?

\section{Lösung:}

a)

b)

Lisa:
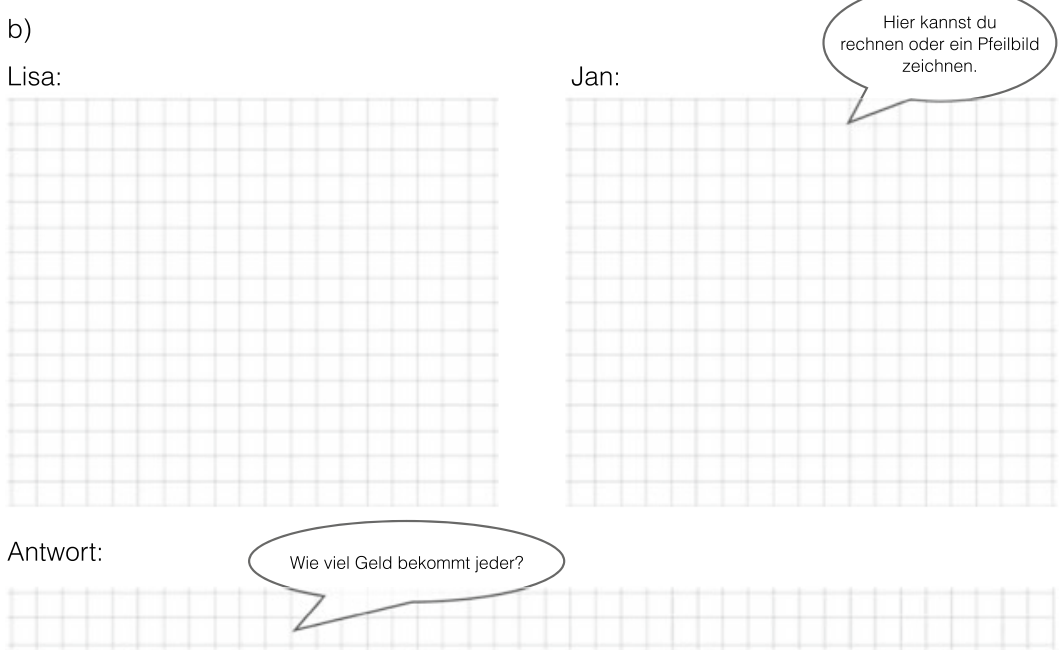

Abbildung 5.21 Unvollständiges Beispiel - Aufgabe 2.2 
Pfeilbild zeichnen“. Es wird den Lernenden somit selbst überlassen, ob sie eine einfache Rechnung oder eine Rechnung im Operator- bzw. Pfeilschema notieren. Zuletzt ist eine Fläche für die Formulierung eines Antwortsatzes vorgegeben.

Transferprozesse im zweiten unvollständigen Beispiel: Obgleich die Aufgabenstellung und auch die Aufgabenlösung analog zum Lösungsbeispiel und dem ersten unvollständigen Beispiel ist, erfordert die Lösung dieser Aufgabe einen weiteren Transfer als noch im ersten unvollständigen Beispiel. Die Gründe dafür sind die folgenden: Zunächst sind anders als im ersten unvollständigen Beispiel keine Teile des Lösungsweges oder Darstellungen zur Ergänzung vorgegeben und die Lernenden müssen alle Lösungsschritte selbst durchführen. Die zweigeteilte Aufgabenstellung sowie die Hinweise in den Sprechblasen sind die einzigen Lösungshilfen für die Lernenden und sollen den Lösungsprozess strukturieren und die Übertragung des Lösungsweges aus dem Lösungsbeispiel und dem ersten unvollständigen Beispiel unterstützen.

Für die Übertragung und Anwendung des Verfahrens sind die Unterschiede zu den vorhergehenden Aufgaben von Relevanz, da sie die Bildung einer Analogie und damit die Anpassung des Lösungsweges erfordern. Erstens wird der Geldbetrag nicht auf zwei Familien aufgeteilt, sondern auf zwei Personen und zweitens ist hier nicht die Anzahl der Personen die Bezugsgröße zur Bildung der Anteile, sondern die Anzahl an geleisteten Arbeitsstunden.

Durch diese Unterschiede lässt sich der Transfer in dieser Aufgabe als Transfer auf Ebene des Sachkontexts charakterisieren. Für die Bildung einer Analogie zum Lösungsbeispiel und dem ersten unvollständigen Beispiel ist es essenziell zu erkennen, dass die Summe der Arbeitsstunden als Einteilung des Ganzen genutzt werden muss. Die Summe der Arbeitsstunden in dieser Aufgabe entspricht der Summe der Familienmitglieder der beiden Familien zuvor. Es wird angenommen, dass diese Abbildung bei einigen Schülerinnen und Schülern zu Schwierigkeiten führt, da unterschiedliche Bezugsgrößen miteinander in Beziehung gesetzt werden müssen. Es besteht somit die Möglichkeit, dass eine fehlerhafte Analogie hergestellt wird, die zu einem falschen Ergebnis bzw. einer ungerechten Aufteilung des Geldbetrags führen kann.

Zusammenfassend sind die für die Lösung dieser Aufgabe erforderlichen Transferprozesse im Rahmen des Übertragens und Anwendens des Verfahrens aus dem Lösungsbeispiel und dem ersten unvollständigen Beispiel zu beschreiben. Im Spezifischen betrifft das die folgenden Prozesse: 
- Herstellen der Analogie zwischen der Anzahl der Personen der Familien und der Anzahl der geleisteten Arbeitsstunden als Bezugsgröße für die Aufteilung des Ganzen,

- Übertragung und Anwendung des Verfahrens zu Anteilbestimmung und

- Übertragung und Anwendung des Verfahrens zur Berechnung von Anteilen einer Größe.

\section{Bennet \& Julius - Erfolgreicher Transfer des Operatorschemas}

Bennet und Julius haben das Lösungsbeispiel zum Einstieg eingehend gelesen und während des Lesens auch mehrfach innegehalten, um einzelne Lösungsschritte nachzuvollziehen und vereinzelte Unklarheiten zu diskutieren. Dies spiegelt sich auch in ihren Antworten auf die fokussierenden Fragestellungen wieder, in der sie zunächst die Aufteilung des Gewinns auf Grundlage der Anzahl der Personen in jeder Familie erklären und die Berechnung des Anteils für Familie Meyer in Form einer Rechnung beschreiben (vgl. Abb. 5.22).

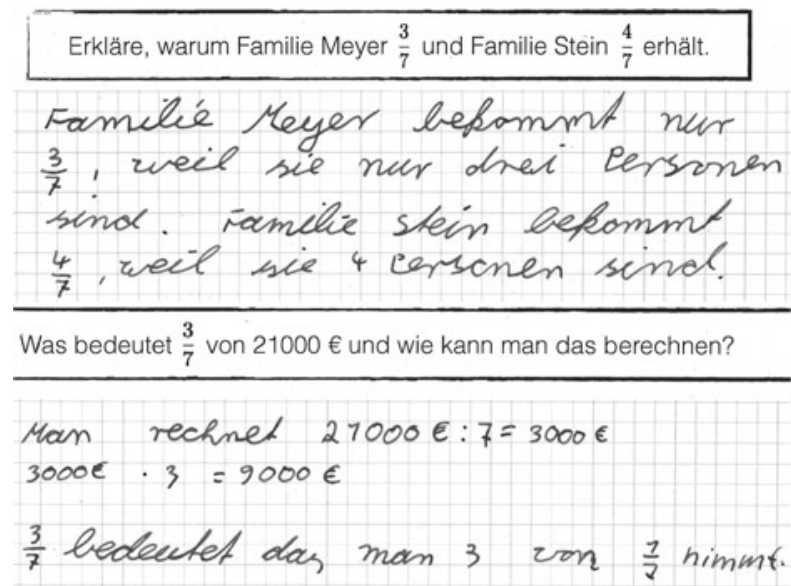

Abbildung 5.22 Julius Antworten auf die fokussierenden Fragen zum Lösungsbeispiel

\section{Transkript B2A1 - Bennet \& Julius - Szene 1 - Aufgabe 1}

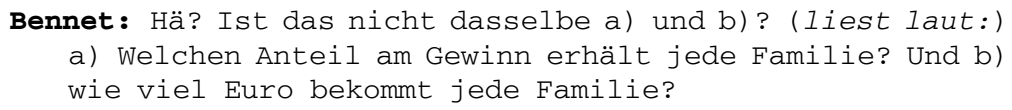




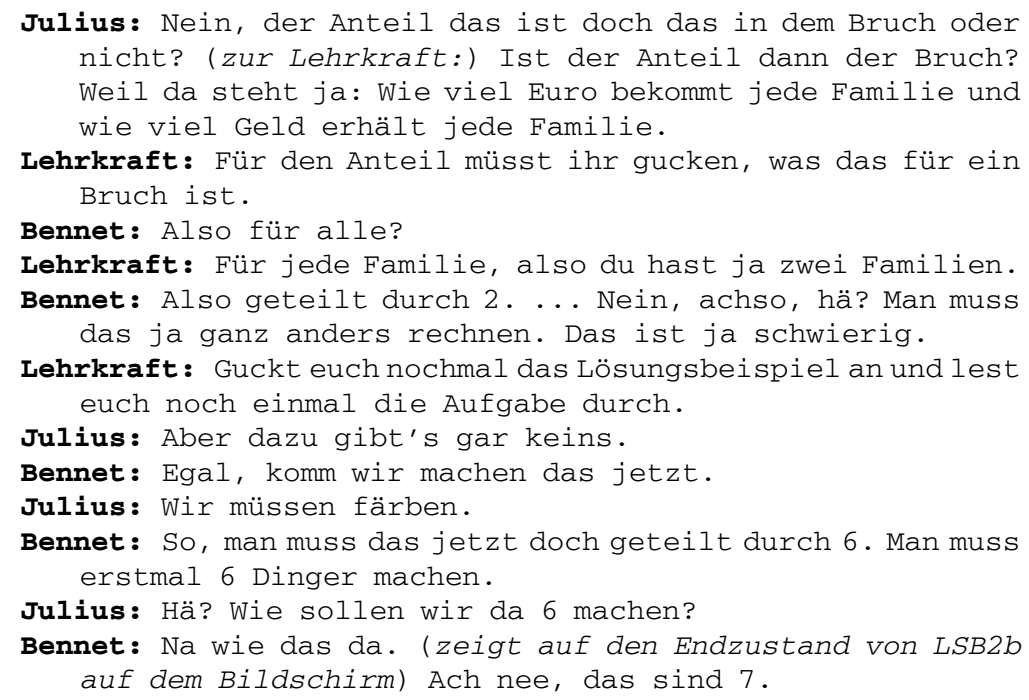
nicht? (zur Lehrkraft:) Ist der Anteil dann der Bruch? Weil da steht ja: Wie viel Euro bekommt jede Familie und wie viel Geld erhält jede Familie.

Lehrkraft: Für den Anteil müsst ihr gucken, was das für ein Bruch ist.

Bennet: Also für alle?

Lehrkraft: Für jede Familie, also du hast ja zwei Familien. Bennet: Also geteilt durch 2.... Nein, achso, hä? Man muss das ja ganz anders rechnen. Das ist ja schwierig.

Lehrkraft: Guckt euch nochmal das Lösungsbeispiel an und lest euch noch einmal die Aufgabe durch.

Julius: Aber dazu gibt's gar keins.

Bennet: Egal, komm wir machen das jetzt.

Julius: Wir müssen färben.

Bennet: So, man muss das jetzt doch geteilt durch 6 . Man muss erstmal 6 Dinger machen.

Julius: Hä? Wie sollen wir da 6 machen?

Bennet: Na wie das da. (zeigt auf den Endzustand von LSB2b auf dem Bildschirm) Ach nee, das sind 7 .

Der Beginn der Bearbeitung von Bennet und Julius ist auf den ersten Blick von der Klärung der Aufgabenstellung geprägt. Bennet sieht direkt nach dem Lesen der Aufgabenstellung keinen Unterschied zwischen den Teilaufgaben a) und b): „Ist das nicht dasselbe?“" (1). Im weiteren Verlauf ist jedoch zu erkennen, dass die Klärung der Aufgabenstellung die Abgrenzung des Begriffs Anteil voraussetzt. Julius erwidert, dass mit dem Begriff Anteil die Angabe eines Bruchs gemeint ist. Er versichert sich dazu bei der Lehrkraft: „Ist der Anteil dann der Bruch?“ (5), was die Lehrkraft bestätigt und erklärt, dass sie für die Angabe des Anteils überlegen müssen, ,was das für ein Bruch ist" (8-9). Bennet fragt daraufhin, ob sie die Anteile für ,alle“ (10) Personen bestimmen sollen, worauf die Lehrkraft verweist, dass sie überlegen sollen welchen Anteil jede der zwei Familien bekommt (11). Mit Blick auf die zwei Familien schlägt Bennet spontan vor, den Gewinn ,durch 2“ (12) zu teilen, bemerkt dann aber umgehend, dass sie ,ja ganz anders rechnen“ müssen, was ,ja schwierig“ (13) sei.

$\mathrm{Ab}$ diesem Moment scheint Bennet zu wissen, wie sie vorgehen müssen: „So, man muss das jetzt doch geteilt durch 6“ (19) rechnen. Bezüglich der Veranschaulichung der Anteile im Kreisdiagramm sagt er, dass sie ,erstmal 6 Dinger machen“ (20) müssen, womit er meint, dass sie den Kreis in sechs gleiche Teile einteilen müssen.

Die erste Szene der Bearbeitung von Bennet und Julius macht deutlich, dass selbst nach intensivem Lesen und Diskutieren des animierten Lösungsbeispiels und der 
ausführlichen Beantwortung der fokussierenden Fragestellungen, die Übertragung des Vorgehens nicht einfach ist. Beide Schüler stellen zunächst keine Beziehung zum Lösungsbeispiel her und Julius erwidert auf den Hinweis der Lehrkraft, noch einmal in das Lösungsbeispiel zu schauen, dass es zu dieser Aufgabe ,gar keins“ gebe (16). Auch Bennet stellt zunächst keine Verbindung zum Vorgehen im Lösungsbeispiel her. Erst als er selber vorschlägt, dass der Geldbetrag durch zwei geteilt werden müsse, stellt er eine Verbindung zum Lösungsbeispiel her und erkennt, dass das Ganze durch Summe der Anzahlen an Familienmitgliedern geteilt werden muss. Es ist anzunehmen, dass er sich durch seinen Vorschlag den Gewinn in zwei gleiche Teile zu teilen an das Lösungsbeispiel erinnert, in dem der Gewinn ebenfalls gerecht auf zwei Familien aufgeteilt werden sollte, dabei jedoch nicht halbiert, sondern auf die Anzahl an Personen aufgeteilt wurde.

Es sind verschiedene Gründe denkbar, warum die Schüler die Analogie zum Lösungsbeispiel nicht sofort herstellen. Zum einen unterscheidet sich die visuelle Gestaltung der Aufgabe deutlich von der Darstellung im Lösungsbeispiel. Besonders hervorzuheben ist hierbei, dass die Aufgabenstellung keine Abbildungen der Familien bzw. Familienmitglieder enthält, während die Personenabbildungen im Zentrum der Darstellung des Vorgehens im Lösungsbeispiel stehen. Entsprechend stellen Bennet und Julius nicht direkt einen Zusammenhang zwischen der Anzahl der Personen und der Anzahl der Teile her, in die das Ganze geteilt werden muss. Zum anderen unterscheiden sich die enthaltenen Zahlen im Lösungsbeispiel und im ersten unvollständigen Beispiel deutlich. Während im Lösungsbeispiel ein verhältnismäBig großer Geldbetrag von $21000 €$ aufgeteilt wird, ist es im ersten unvollständigen Beispiel ein Betrag von $900 €$.

\section{Transkript B2A1 - Bennet \& Julius - Szene 2 - Aufgabe 1}

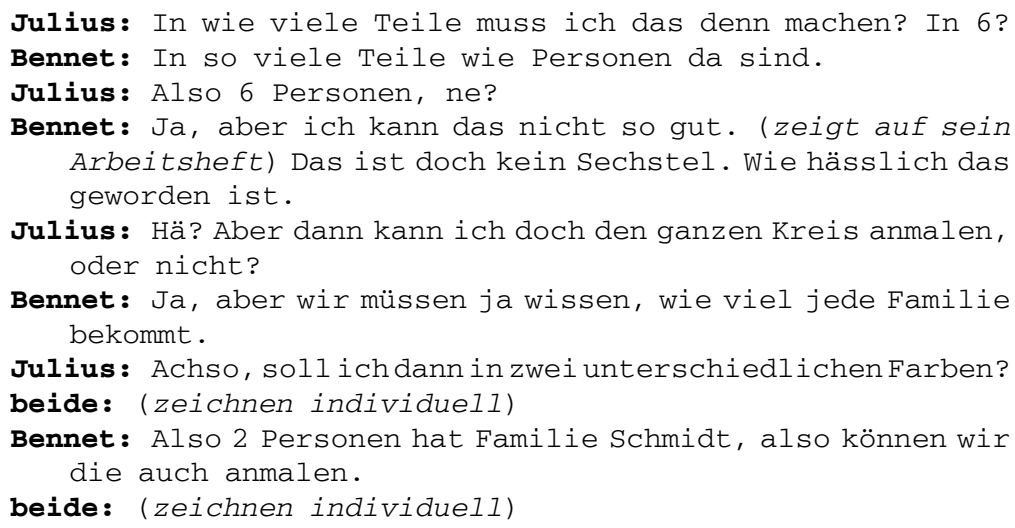




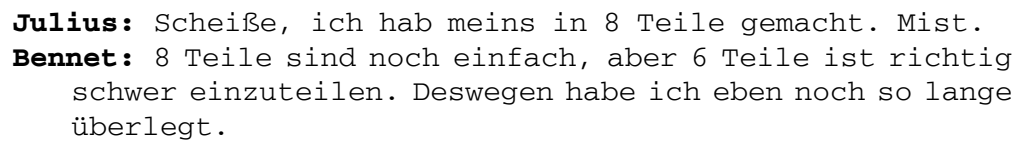

Bennet und Julius beginnen ihre Bearbeitung mit der ikonischen Darstellung der Anteile für jede Familie. Auf Julius Rückfrage, ob sie den Kreis ,in 6“ (25) Teile sollen, stellt Bennet noch einmal heraus, dass sie das Ganze ,in so viele Teile wie Personen da sind“ (26) teilen müssen. Diese Äußerung stützt die Annahme, dass er die Analogie zum Lösungsbeispiel aufgrund der fehlenden Personendarstellung in der Aufgabe nicht sofort herstellen konnte. In der Abbildung der Aufgabe sind die Personen nicht zu sehen, sondern lediglich den Zahlen im Aufgabentext zu entnehmen.

Im Gegensatz zu seinem Partner scheint Julius die Verbindung zum Lösungsbeispiel noch nicht vollständig hergestellt zu haben. Nachdem er die Kreisrepräsentation in sechs gleiche Teile eingeteilt hat, stellt er irritiert fest, dass er ,,doch den ganzen Kreis anmalen“ (31-32) könne. Es kann angenommen werden, dass er erkannt hat, dass die Familien zusammen sechs Personen haben, jedoch nicht erkannt hat, dass die Teile dann entsprechend der Personenanzahl der beiden Familien verteilt werden, also eine Familie zwei Teile und eine Familie vier Teile bekommt. Auf den Hinweis von Bennet, dass sie ,wissen [müssen], wie viel jede Familie bekommt“ (33-34) schlägt er dann jedoch vor die Anteile ,in zwei unterschiedlichen Farben“ (35-36) einzuzeichnen.

Das Einteilen der Kreisrepräsentation in sechs gleiche Teile gestaltet sich für beide Schüler schwierig. Beim Einfärben der Teile fällt Julius auf, dass er das Ganze nicht in sechs, sondern in acht Teile eingeteilt hat (41). Auch Bennet erklärt, dass das Einteilen in 8 Teile ,noch einfach“ sei, aber ,6 Teile [...] richtig schwer einzuteilen“ sei, weswegen er ,eben noch so lange überlegt“ (42-44) habe. Diese Äußerung von Bennet deutet darauf hin, dass er die Darstellung der Anteile vor dem Einzeichnen zunächst in seiner Vorstellung simuliert hat und somit beim Einzeichnen auf dem Papier mit einer Strategie vorgegangen ist. Im Gegensatz dazu ist anzunehmen, dass Julius versucht hat durch sukzessives Halbieren sechs Teile herzustellen, was zu seiner Einteilung des Ganzen in acht gleiche Teile geführt hat.

\section{Transkript B2A1 - Bennet \& Julius - Szene 3 - Aufgabe 1}


Nachdem Bennet und Julius jeweils die Anteile für beide Familien in unterschiedlichen Farben eingezeichnet haben, beginnen sie mit der Berechnung der Anteile. Bennet scheint sofort zu wissen, wie er den Gewinn der beiden Familien berechnen kann (45). Er rechnet schriftlich $900: 6$ (47-48) und erklärt, dass $150 €$ einem Sechstel des Gewinns entsprechen (53-54). Für den Anteil von Familie Schmidt multipliziert er „150Euro mal 2 gleich 300Euro. Das ist jetzt Familie Schmidt“ (58-59). Es ist deutlich zu erkennen, dass Bennet die Berechnung der Anteile durch Anwendung des Operatorschemas leicht fällt, was auch durch seine Bemerkung „Das ist einfach“ (45) gestützt wird. In seinem Rechenweg ist deutlich die Übersetzung des Anteils in die Hintereinanderausführung von zwei Teiloperatoren zu erkennen.

Im Gegensatz zu seinem Partner scheint Julius nicht sofort zu wissen, wie man die Anteile am Gewinn ausrechnet. Auf die Aussage von Bennet, dass er das ,jetzt erstmal aus[rechnet]““ (51) reagiert Julius mit der Frage „Wie?“ (52). Im weiteren 
versucht er dem schnellen Vorgehen von Bennet zu folgen und versichert sich der einzelnen Rechenschritte und Zwischenergebnisse $(55,57)$. In seinen Nachfragen ist zu erkennen, dass er sich bezüglich der erforderlichen Rechenschritte sehr unsicher ist. Er teilt wie sein Partner $900 €$ durch 6 und kommt ebenfalls zu dem Ergebnis $150 €$. Er folgert dann jedoch, dass diese $150 €$ bereits den Anteil für Familie Schmidt darstellen, woraufhin Bennet ihm erklärt: „Du musst das ja noch mal 2 rechnen, weil du hast ja jetzt erst 1 Sechstel ausgerechnet, aber wir brauchen 2 Sechstel“ (64-66). Auch bei der Berechnung des Anteils für Familie Tenner scheint Julius unsicher zu sein, was er rechnen muss. Er bittet seinen Partner ihm zu zeigen, wie er das gerechnet hat (70) und weiß auch auf die Erwiderung von Bennet, dass er „mal 4“ (71) gerechnet habe nicht, worauf der den Operator 44 anwenden soll: „Also 300 Euro mal 4? Oder 150?“ (72).

Es ist denkbar, dass Julius Schwierigkeiten bei der Anteilberechnung auf die hohe Geschwindigkeit der Bearbeitung seines Partners zurückzuführen ist und er mehr Zeit zur Herstellung der Analogie zum Lösungsbeispiel und zum Ableiten der entsprechenden Rechenoperationen benötigt. Hinzu kommt, dass die beiden Schüler die Berechnung der Anteile bereits in Teilaufgabe a) vornehmen und sich noch nicht die vorgegebenen Pfeilschemata in Teilaufgabe b) angeschaut haben (vgl. Abb. 5.23). An dieser Stelle kann angenommen werden, dass ihm die Berechnung der Anteile in einem Pfeildiagramm helfen würde.

\section{Transkript B2A1 - Bennet \& Julius - Szene 4 - Aufgabe 1}

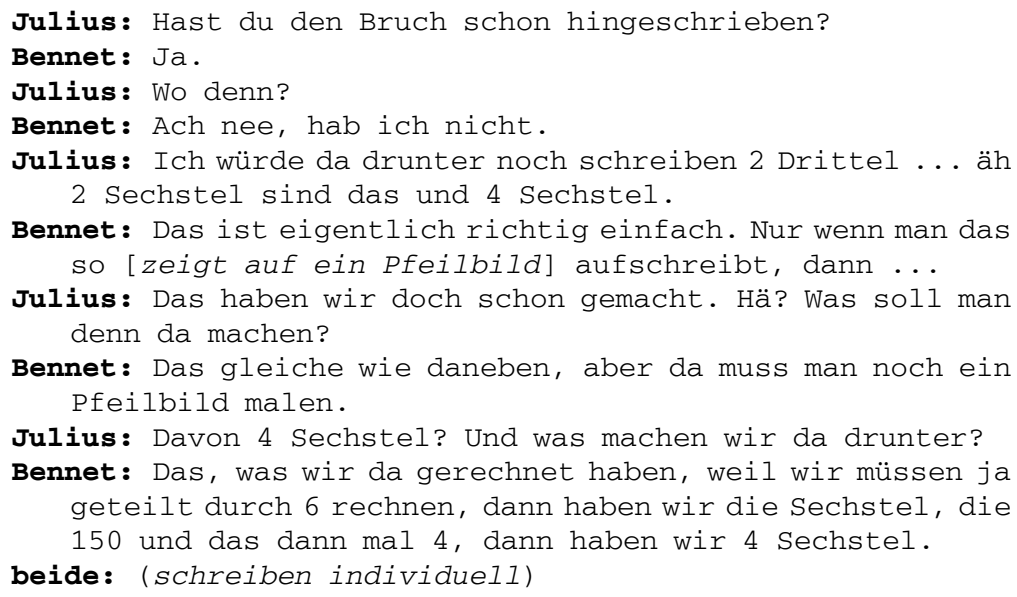


a) 900 Euro sind das ganze gewonnene Geld.

Es wird in 6 gleich große Teile zerlegt.

Jeder Teil ist ein Sechstel des Gewinns.

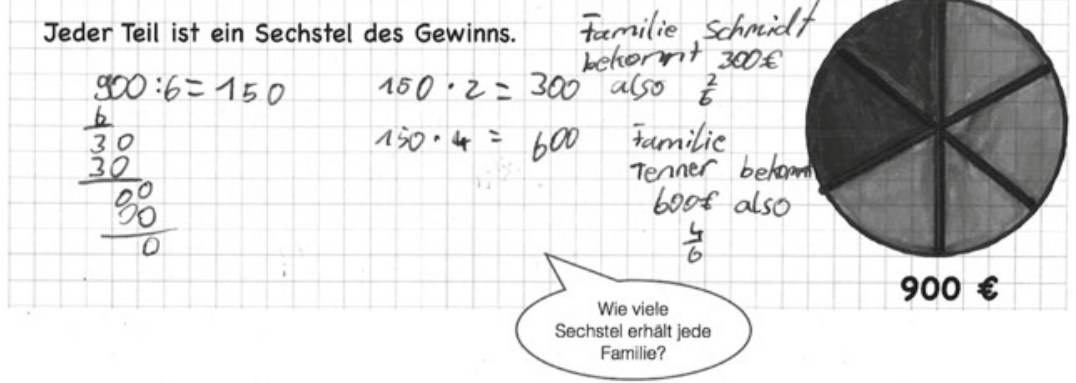

b) Familie Schmidt:

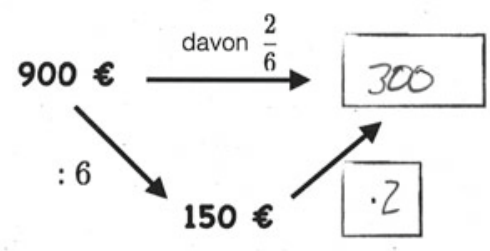

Familie Tenner:

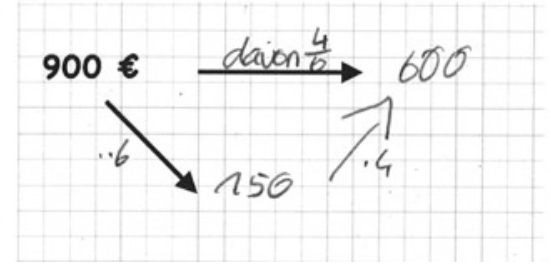

Abbildung 5.23 Bennets Lösung von Aufgabe 2.1

Als die beiden Partner im Anschluss zu Teilaufgabe b) übergehen, ist Julius zunächst irritiert: „Das haben wir doch schon gemacht. Hä? Was soll man denn da machen?“ (84-85). Er erkennt scheinbar, dass das Pfeilschema der Berechnung der Anteile dient, weshalb er feststellt, dass sie das schon gemacht haben. Entsprechend wundert er sich, was sie dann mit den Pfeildiagrammen machen sollen.

Bennet erkennt, dass sie mit der Berechnung der Anteile dem Aufgabenteil b) bereits vorgegriffen haben. Er erklärt, dass er die Berechnung von Anteilen ,eigentlich richtig einfach“ (82) findet, jedoch die Darstellung der Rechnung in einem Pfeildiagramm nicht als Hilfe, sondern als Erschwernis empfindet (82-83). Er weiß jedoch sofort, was an den entsprechenden Stellen im Pfeildiagramm eingetragen werden muss und erklärt: „Das, was wir da gerechnet haben, weil wir müssen ja geteilt durch 6 rechnen, dann haben wir die Sechstel, die 150 und das dann mal 4, dann haben wir 4 Sechstel“" (89-91). Es ist anzunehmen, dass er die Darstellung im Pfeildiagramm als erschwerend empfindet, weil er sie nicht als Hilfe benötigt und auch ohne die Darstellung, weiß was er rechnen muss. Vor diesem Hintergrund 
ist anzunehmen, dass er somit für die Darstellung des Pfeilschemas zusätzlich zu den erforderlichen Rechenoperatoren noch darüber nachdenken muss, wie diese Rechnung im Diagramm dargestellt werden muss, wodurch diese Darstellung für ihn lediglich einen zusätzlichen Aufwand bedeutet.

Obwohl sie die entsprechenden Anteile bereits in Teilaufgabe a) berechnet und die erforderlichen Rechenoperatoren und Ergebnisse berechnet haben, scheint Julius diese nicht auf die Darstellung im Pfeildiagramm übertragen zu können, sodass er seinen Partner fragt, was sie dort eintragen müssen. Diese Beobachtung entkräftet die Annahme, dass er zuvor lediglich Schwierigkeiten hatte, der Geschwindigkeit seines Partners zu folgen. Wäre dem so gewesen, wäre anzunehmen gewesen, dass er ohne Schwierigkeiten die entsprechenden Ergänzungen im Pfeildiagramm hätte vornehmen können. Stattdessen sucht er jedoch Hilfe bei seinem Partner und übernimmt dessen Lösung.

Die Interpretation von Bennet und Julius Bearbeitung von Aufgabe 1 kann in den folgenden Deutungshypothesen zusammengefasst werden:

- Die Schüler haben die Analogie zum Lösungsbeispiel erst nach einem Hinweis von der Lehrkraft hergestellt. Obgleich die Aufgabenstellung mit Ausnahme der Zahlenwerte nahezu identisch zur Aufgabenstellung im Lösungsbeispiel ist, erkennen Bennet und Julius zunächst keinen Zusammenhang. Julius merkt sogar an, dass es für diese Aufgabe kein Lösungsbeispiel gebe. Erst im Austausch mit der Lehrkraft, die den Fokus der Schüler auf den Kontext der Aufgabe und im Speziellen auf das Aufteilen eines Geldbetrags auf zwei Familien lenkt, stellt Bennet eine Beziehung zum Lösungsbeispiel her und bildet die einzelnen Lösungsschritte auf die Bearbeitung des unvollständigen Beispiels ab. Es kann vermutet werden, dass die äußere Gestaltung der Aufgabe, die sich sehr vom Lösungsbeispiel unterscheidet, der wesentliche Grund für die zunächst getrennte Betrachtung von Bennet und Julius ist. Da im Lösungsbeispiel durch die Abbildung von Personen ein direkter Zusammenhang zwischen den Teilen des Ganzen und den Familienmitgliedern dargestellt wird, sind im ersten unvollständigen Beispiel keine Personen abgebildet. Dies hat womöglich zur Folge, dass die Personenanzahl nicht direkt als Aufteilungsgrundlage erkannt und somit zunächst kein Bezug zum Lösungsbeispiel hergestellt wird.

- Das Einzeichnen von Sechsteln im Kreisdiagramm fällt beiden Schülern schwer. Bennet und Julius sind sich schnell einig, dass sie die Kreisrepräsentation in insgesamt sechs gleich große Teile einteilen müssen. Die Durchführung gestaltet sich jedoch nicht so einfach. Während Julius versucht über ein schrittweises Halbieren der Kreissegmente die Einteilung herzustellen und so den Kreis in acht anstelle von sechs gleichen Teilen einteilt, erklärt Bennet, dass er „lange“ 
über die Einteilung nachdenken musste. Beiden Schülern ist an einer möglichst genauen Einteilung gelegen. Da die Einteilung in Sechstel nicht durch fortgesetztes Halbieren des Kreises und der Kreissegmente hergestellt werden kann, ist es notwendig im Vorhinein entweder eine genaue Vorstellung von dem Ergebnis der Einteilung oder eine Strategie zum Einzeichnen zu haben, z.B. Einteilen in Drittel und Halbieren der Drittel. Beides setzt die mentale Simulation des Vorgehens oder eine mentale Repräsentation voraus.

- Das Operatorschema wird sowohl auf die Bildung wie auch auf die Berechnung der Anteile übertragen und angewendet. Nachdem Bennet den Zusammenhang zum Lösungsbeispiel hergestellt hat, gelingt es ihm zunächst die Anteile für beide Familien zu bestimmen, indem er das Ganze in sechs gleiche Teile aufteilt und diese Teile den einzelnen Familien zuordnet. Auch die Berechnung des Gewinns für beide Familien stellt für ihn keine Schwierigkeit dar. Er wendet die korrekten Lösungsschritte und Rechenoperationen an, ohne dass er sich dabei am Lösungsbeispiel orientieren muss bzw. auf dieses zurückgreifen muss, was so interpretiert werden kann, dass er das Operatorschema bereits verinnerlicht hat und flexibel auf neue Anforderungen übertragen kann. Im Gegensatz zu Bennet zeigt Julius Unsicherheiten bezüglich der Anwendung des Operatorschemas. Seine Unsicherheiten betreffen die Bestimmung der erforderlichen Teiloperatoren und auf welche Größen diese angewendet werden müssen. Dennoch gelingt es ihm, dem Lösungsweg seines Partners zu folgen und die Lösung selbstständig zu notieren.

- Bennet äußert, dass die Anwendung des Operatorschemas zur Berechnung von Anteilen ,eigentlich einfach“ sei, er jedoch die Darstellung im Pfeilschema als schwierig oder aufwändig empfindet. Es ist anzunehmen, dass er das Vorgehen zur Bruchherstellung und Anteilberechnung bereits soweit verallgemeinert hat, dass er direkt die erforderlichen Herstellungs- bzw. Rechenschritte ableiten und auf symbolischer Ebene anwenden kann. Die Darstellung in einer gesondert strukturierten Darstellung wie dem Pfeilschema bedeutet in diesem Fall einen zusätzlichen Aufwand, da die Struktur erinnert und zeichnerisch dargestellt werden muss. Im Fall von Julius, der Unsicherheiten bei der Anwendung des Verfahrens zeigt, ist im Gegensatz dazu anzunehmen, dass für ihn eine Darstellung im Pfeilschema eine Unterstützung bei der Strukturierung des Rechenwegs sein könnte. In diesem Zusammenhang ist zu bemerken, dass Bennet und Julius bereits in Teilaufgabe a) die Gewinnanteile der beiden Familien berechnen, ohne die vorgegebenen Pfeilschemata bisher bewusst wahrgenommen zu haben und somit nicht auf ihre Struktur zugegriffen haben. 
Direkt im Anschluss an die Bearbeitung des ersten unvollständigen Beispiels gehen Bennet und Julius zum zweiten unvollständigen Beispiel über.

\section{Transkript B2A2 - Bennet \& Julius - Szene 5 - Aufgabe 2}

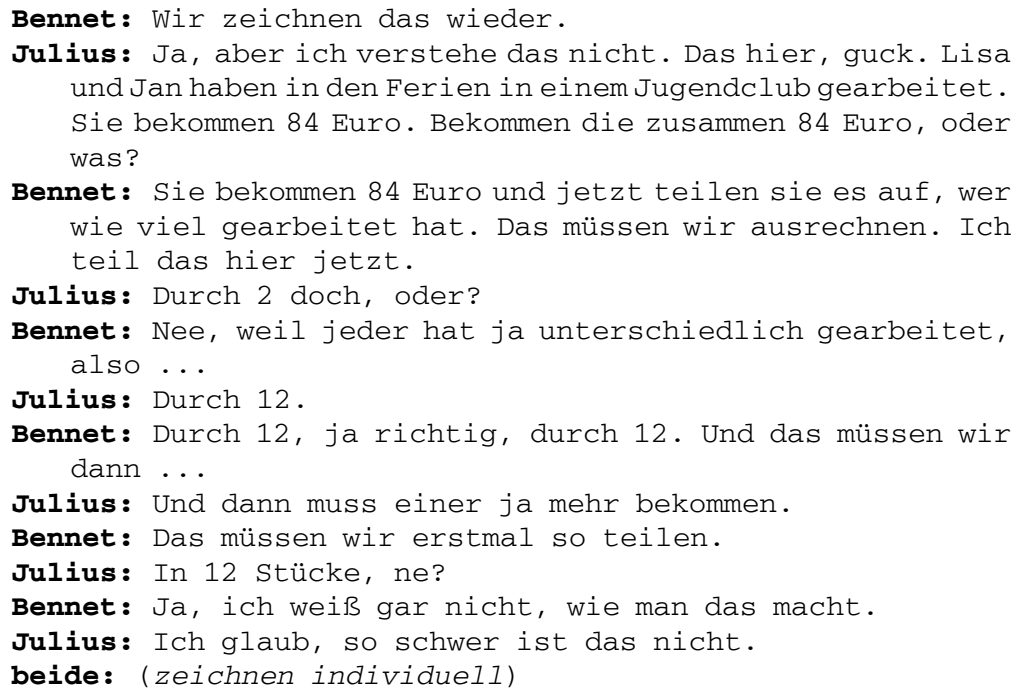

Nach dem Lesen der Aufgabenstellung schlägt Bennet vor, wieder eine Zeichnung anzufertigen (1). Er scheint beim Lesen der Aufgabenstellung bereits verstanden zu haben, was in der Aufgabe zu tun ist und möchte direkt die Aufteilung des Geldbetrags auf die beiden Jugendlichen zeichnerisch veranschaulichen: „Sie bekommen 84 Euro und jetzt teilen sie es auf, wer wie viel gearbeitet hat. Das müssen wir ausrechnen. Ich teil das hier jetzt" (6-8). Bennets Erklärung lässt annehmen, dass er sofort erkannt hat, dass die Summe der Arbeitsstunden der beiden Jugendlichen als Grundlage für die Einteilung des Ganzen genutzt werden muss.

Julius erkennt diesen Zusammenhang hingegen nicht auf Anhieb und sagt, er „verstehe das nicht“ (2). Er entnimmt der Aufgabenstellung, dass die beiden Jugendlichen zusammen $84 €$ bekommen haben, die sie untereinander aufteilen wollen. Entsprechend schlägt er vor, dass der Lohn „,durch 2“ (9) geteilt werden müsse, worin $\mathrm{zu}$ erkennen ist, dass er die unterschiedliche Arbeitszeit der beiden Jugendlichen in seinen Überlegungen nicht berücksichtigt. Auf die Erwiderung von Bennet, dass die beiden Jugendlichen unterschiedlich lange gearbeitet haben (10-11), erkennt Julius seinen Fehler und stellt fest, dass der Lohn dann ,durch 12“ (12) geteilt wer- 
den müsse, was Bennet bestätigt. Es ist anzunehmen, dass Julius Wahl des falschen Ansatzes eher darauf zurückzuführen ist, dass er die Aufgabenstellung nicht direkt in vollem Umfang erfasst hat, als dass er das grundlegende Verfahren nicht verstanden hat. Diese Annahme wird insbesondere dadurch gestützt, dass Julius sich zu Beginn bei seinem Partner versichert, dass die beiden Jugendlichen zusammen einen Lohn ausgezahlt bekommen, was eine eher ungewöhnliche Situation ist, da ein Arbeitslohn im Alltag direkt an die arbeitende Person ausgezahlt wird und dieser nicht erst aufgeilt werden muss. In diesem Zusammenhang kann seine Irritation auf die Erschließung des Aufgabenkontexts zurückgeführt werden. Zudem erkennt er unmittelbar auf den Hinweis von Bennet, dass die beiden Jugendlichen unterschiedlich lange gearbeitet haben, dass der Gewinn durch die Summe der Arbeitsstunden geteilt und das Ganze entsprechend in zwölf gleiche Teile geteilt werden muss. Zudem erkennt er, dass die beiden Jugendlichen nicht denselben Anteil erhalten, sondern „einer ja mehr bekommen“ (15) muss. Die Spontaneität dieser Folgerung deutet darauf hin, dass er sofort einen Bezug zu den vorhergehenden Aufgaben herstellt und das Verfahren auf die neue Situation bzw. die neuen Zahlenwerte anpasst und anwendet.

Im Anschluss teilen die beiden Partner eigenständig die vorgegebene Kreisrepräsentation in zwölf gleiche Teile und färben die Anteile von Lisa und Jan in verschiedenen Farben ein (vgl. Abb. 5.24).

\section{Transkript B2A2 - Bennet \& Julius - Szene 6 - Aufgabe 2}

Julius: Warte, jetzt muss ich ja dann 5 stunden ... hä? Da mach ich doch 5 und 7 , oder? 7 Zwölftel und 7 Fünftel, oder? Äh, 5 Siebtel. Nee, 5 Zwölftel.

Bennet: Du musst jetzt erstmal 5 Stücke und nochmal 7 stücke. beide: (schreiben individuell)

Bennet: Hä? Wir müssen doch hier jetzt den Anteil ausrechnen (schaut bei Julius) Wie bist du auf das gekommen?

Julius: Was?

Bennet: Ach, egal.

Julius: 84 Euro durch 12 sind doch 7 .

beide: (schreiben individuell)

Julius: Dann kommt da ja 7 Euro raus und dann müssen wir 7 $\operatorname{mal} 7 \ldots$

Bennet: Warte doch kurz.

beide: (schreiben individuell)

Julius: Hast du das genauso wie ich? Wenn man 84 durch 12 nimmt, kommt ja 7 Euro raus, ne? Und dann musst du ja, Lisa hat ja 7 Stunden gearbeitet und Jan 5. Dann muss man $7 \mathrm{mal} 7$ Euro sind ja gleich 49 Euro. Also Lisa hat 
Nachdem beide Schüler ohne Schwierigkeiten die entsprechenden Anteile eingezeichnet haben versucht Julius die Anteile zu benennen: „Warte, jetzt muss ja dann 5 Stunden ... hä? Da mach ich doch 5 und 7, oder? 7 Zwölftel und 7 Fünftel, oder? Äh, 5 Siebtel. Nee, 5 Zwölftel“" (21-23). Obwohl er die Anteile korrekt eingeteilt und markiert hat, wirkt Julius zunächst ein wenig irritiert, was die Einheit bzw. der Nenner der Brüche ist. Er hat erkannt, dass die eine Person fünf Stunden und die andere Person sieben Stunden bezahlt bekommt (21) und entsprechend die Zähler der Brüche 5 und 7 sein müssen (22). Nach einigen Versprechern nennt er dann korrekt die Anteile $\frac{7}{12}$ und $\frac{5}{12}$. Bennet bestätigt dies, indem er auf die Einteilung der Kreisfläche verweist: „Du musst erstmal 5 Stücke und nochmal 7 Stücke“ (24), wobei er sich mit „Stücke“ auf die Teile des Ganzen, also Zwölftes bezieht.

In der Folge rechnen beide Schüler in individueller Stillarbeit die entsprechenden Lohnanteile für die beiden Jugendlichen aus. Dabei zeigt Julius, dass er das Operatorschema zur Anteilberechnung sicher anwenden kann. Er beschreibt seine Rechnung: „84 Euro durch 12 sind doch 7. [...] Dann kommt da ja 7 Euro raus und dann müssen wir 7 mal 7 “ $(30,32-33)$. Um sich der Richtigkeit seiner Lösung zu versichern wiederholt er schließlich noch einmal den ganzen Rechenweg für die Anteile beider Schüler. Dabei formuliert er: ,Wenn man 84 durch 12 nimmt, kommt ja 7 Euro raus, ne? [...]Lisa hat ja 7 Stunden gearbeitet und Jan 5. Dann muss man 7 mal 7 Euro [...] Also Lisa hat 49Euro bekommen und bei Jan ist es ja auch so und da nur einfach mal 5 mal 7. Da kommt 35 Euro raus“ (36-41). Hier ist deutlich zu erkennen, dass er die Operatoren zur Anteilberechnung (:12 und $\cdot 7$ bzw. .5) korrekt anwendet und ihre Wirkung im Kontext interpretiert. Bennet bestätigt die Richtigkeit von Julius Rechnungen und sagt, dass er ,genauso“ (42) gerechnet habe.

Zuletzt setzt Julius zu einer Proberechnung an und beginnt: „Und wenn man beides addiert kommt da...“ (43). Er wird jedoch von Bennet unterbrochen, der bereits mit der Bearbeitung der nächsten Aufgabe beginnt. An dieser Stelle zeigt sich, dass Julius verstanden hat, dass die beiden Anteile zusammen wieder das Ganze, also $84 €$ ergeben müssen und er folglich die Anteile jeweils im Bezug zum Ganzen sieht und den Zusammenhang zwischen Anteilen und Ganzem im Sachkontext herstellt.

Für die Darstellung ihrer Rechnung verzichten Bennet und Julius auf eine Darstellung im Pfeilschema und schreiben die beiden Rechnungsschritte als Terme 
untereinander. Die Möglichkeit der Erstellung eines Pfeilschemas wird in ihrer Bearbeitung nicht erwähnt.

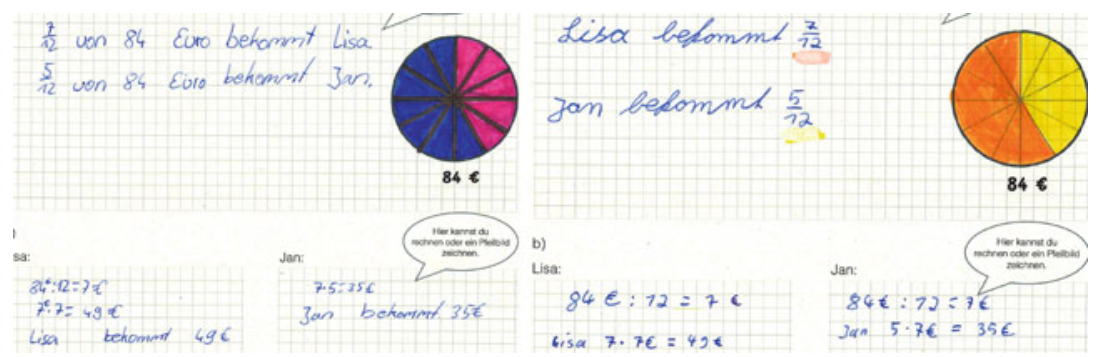

Abbildung 5.24 Bennets (links) und Julius (rechts) Lösung von Aufgabe 2.2

Bennet und Julius Bearbeitung des zweiten unvollständigen Beispiels kann zusammenfassend in den folgenden Deutungshypothesen beschrieben werden:

- Die ungewöhnliche Sachsituation führt zu Verständnisschwierigkeiten. Julius ist zunächst irritiert von der Sachsituation des zweiten unvollständigen Beispiels. Dies betrifft insbesondere die Beschreibung, dass die beiden Jugendlichen gemeinsam einen Lohn ausgezahlt bekommen. Julius ist sich zunächst unsicher, ob er die Aufgabe richtig versteht.

- Bennet und Julius übertragen das Operatorschema erfolgreich zur Bestimmung und zur Berechnung der jeweiligen Anteile am Geldbetrag. Wohingegen in Julius Bearbeitung des ersten unvollständigen Beispiels zunächst Unsicherheiten bezüglich der Bestimmung und Anwendung der Teiloperatoren beobachtet werden konnten, sind in seiner Bearbeitung des zweiten unvollständigen Beispiels keine derartigen Schwierigkeiten zu erkennen. Dies kann als Zeichen gedeutet werden, dass er mit dem Verfahren zunehmend vertraut wird.

- Beide Schüler verzichten in ihrer Notation des Rechenwegs auf die Darstellung in einem Pfeilschema, sondern notieren den Rechenweg in Form von zwei Termen. Es ist anzunehmen, dass Bennet und Julius das Verfahren soweit verallgemeinert haben, dass sie auf das Pfeildiagramm zur Strukturierung der Anteilberechnung nicht angewiesen sind und es im Gegenteil als zusätzliche Erschwernis betrachten. 


\section{Can \& Philip - Probleme bei der Übertragung und Anwendung des Verfah- rens}

Can und Philip Arbeitsverhalten kann als unkonzentriert charakterisiert werden. Insbesondere Can versucht häufig seinen Partner sowie die benachbarten Paare abzulenken. Das Lösungsbeispiel haben sie schnell überflogen, ohne über einzelne Lösungsschritte zu sprechen. Die fokussierenden Fragestellungen wurden im Wesentlichen von Philip bearbeitet (vgl. Abb. 5.25), der die Antworten im Endzustand des Lösungsbeispiels am Bildschirm gesucht hat. Can hat die Antworten unkommentiert von Philip abgeschrieben. Im Anschluss gehen sie zur Bearbeitung des ersten unvollständigen Beispiels über.

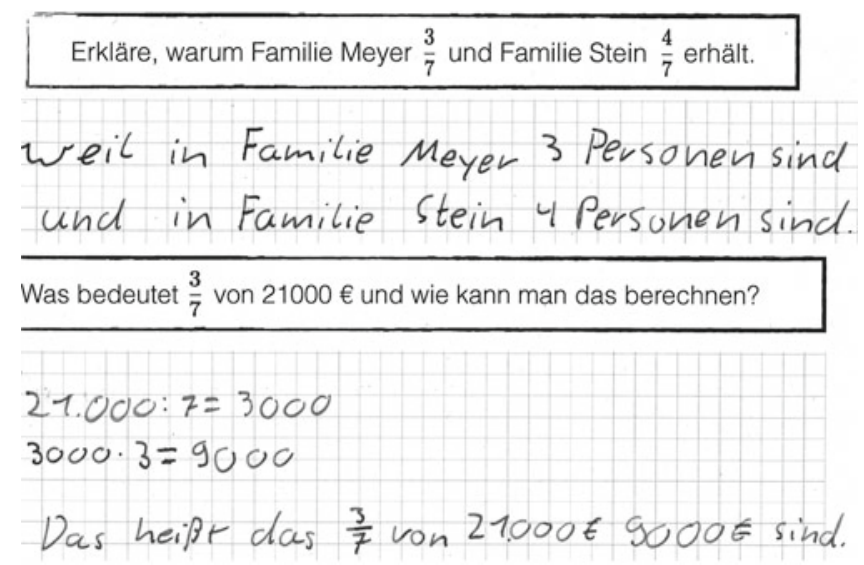

Abbildung 5.25 Philips Antworten zu den fokussierenden Fragestellungen zum Lösungsbeispiel

\section{Transkript B2A1 - Can \& Philip - Szene 1 - Aufgabe 1}

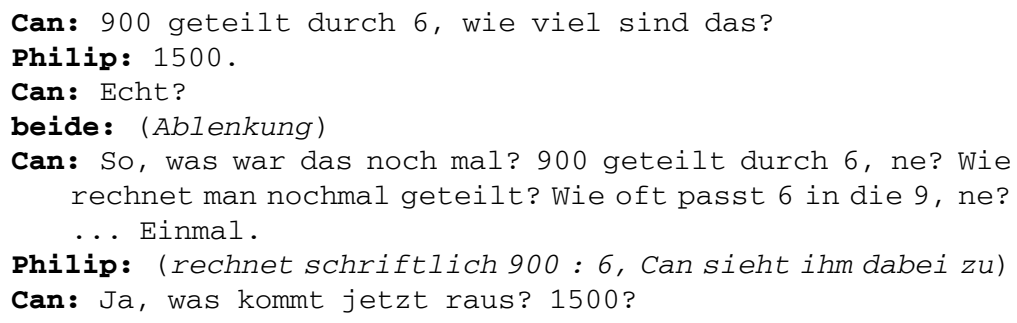




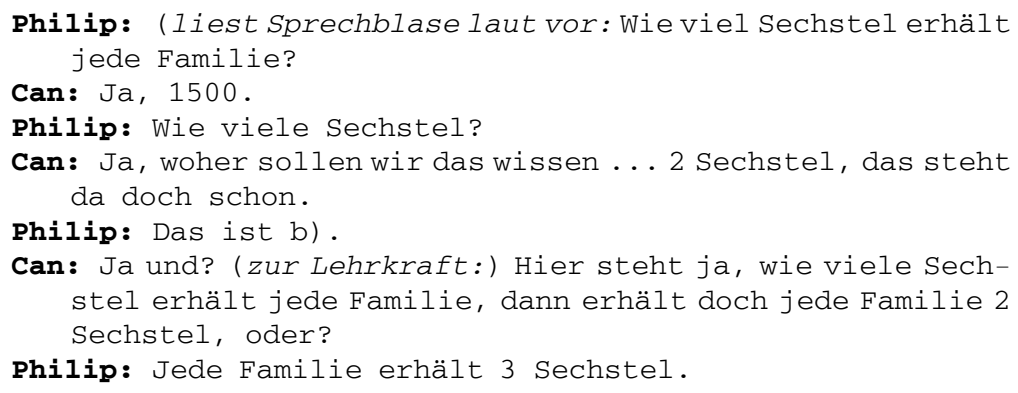

Philip: (liest Sprechblase laut vor: Wie viel Sechstel erhält jede Familie?

Can: Ja, 1500 .

Philip: Wie viele Sechstel?

Can: Ja, woher sollen wir das wissen ...2 Sechstel, das steht da doch schon.

Philip: Das ist b) .

Can: Ja und? (zur Lehrkraft:) Hier steht ja, wie viele Sechstel erhält jede Familie, dann erhält doch jede Familie 2 Sechstel, oder?

Philip: Jede Familie erhält 3 Sechstel.

Nachdem Can und Philip die Aufgabenstellung gelesen haben fragt Can umgehend wie viel ,900 geteilt durch 6“ (1) ist, was Philip mit ,1500“ (2) beantwortet. Es folgt eine Phase der Ablenkung. Cans Frage deutet darauf hin, dass er nicht der Aufgabenstellung entsprechend die Anteile am Gewinn für jede Familie bestimmen möchte, sondern sich mit seiner Frage auf das vorgegebene Pfeildiagramm in Aufgabenteil b) bezieht und dort eine Rechnung herausgreift.

Als die beiden Schüler sich wieder der Aufgabe zuwenden, geht Can erneut auf diese Rechnung ,900 geteilt durch 6“ ein und fragt seinen Partner, wie man eine solche Division durchführt (5-7). Philip hilft ihm und beginnt schriftlich $900: 6$ zu rechnen. Er führt diese Rechnung jedoch nicht vollständig aus, sondern liest die Sprechblase mit dem Bearbeitungshinweis ,Wie viel Sechstel erhält jede Familie?“ (10-11). Can antwortet direkt mit „1500“ (12). In dieser Sequenz wird deutlich, dass die beiden Schüler das erste unvollständige Beispiel nicht mit dem Lösungsbeispiel in Verbindung setzen, sondern versuchen den unvollständigen Lösungsweg auf Grundlage der dargebotenen Informationen zu ergänzen. Erst als Philip den Bearbeitungshinweis liest beginnen sie mit der Bestimmung der Anteile für die zwei Familien. Can scheint mit dem Begriff „Anteil“" keinen Bruch zu verbinden, sondern wiederholt Philips Antwort auf seine Frage, wie viel 900 : 6 sei.

Philip gibt Can zu verstehen, dass „1500“ keine „Sechstel“ sind, indem er die Frage wiederholt: „Wie viele Sechstel?“"(13). Can erwidert, dass sie das nicht wissen können und greift dann den Anteil $\frac{2}{6}$ aus dem unvollständigen Pfeildiagramm in Aufgabenteil b) auf (14-15). Can wendet sich an die Lehrkraft und fragt diese, ob jede Familie 2 Sechstel erhalte (17-19), was Philip mit ,jede Familie erhält 3 Sechstel“" (20) erwidert bevor die Lehrkraft auf Cans Frage antworten kann.

Insgesamt wird der Eindruck verstärkt, dass Can im vorliegenden Aufgabenmaterial nach Lösungen bzw. Rechenwegen sucht, ohne auf die Aufgabenstellung einzugehen. Im Gegensatz dazu greift Philip die Information auf, dass das Ganze auf zwei Familien aufgeteilt werden soll. Er folgert daraus, dass jede Familie den glei- 
chen Anteil „3 Sechstel“ (20) erhält. Es fällt auf, dass die beiden Schüler an keiner Stelle das Teilen des Gewinns durch sechs hinterfragen oder reflektieren. Entsprechend ist weiterhin anzunehmen, dass sie keine Verbindung zum Lösungsweg im Lösungsbeispiel herstellen, sondern im Aufgabenmaterial nach einem Lösungsweg suchen. Dabei nutzen sie keine erkennbaren Strukturen, sondern befolgen die Hinweise im Arbeitsheft.

\section{Transkript B2A1 - Can \& Philip - Szene 2 - Aufgabe 1}

Die Lehrkraft weist Can und Philip darauf hin, dass sie sich noch einmal "genau“ das Lösungsbeispiel anschauen sollen, da dieses nämlich „,fast genauso“ sei (2125). Sie erklärt zudem, dass sie sich das Lösungsbeispiel nicht nur anschauen sollen, sondern versuchen sollen, die einzelnen Lösungsschritte nachzuvollziehen (25-27). Auf die Aufforderung der Lehrkraft klicken Can und Philip sich erneut über die Vorspultaste durch das Lösungsbeispiel, ohne dass zu erkennen ist, dass sie den Text im Lösungsbeispiel lesen, und bleiben bei Philips Annahme, dass beide Familien den gleichen Anteil $\frac{3}{6}$ bekommen. Erneut stellen sie keinen Bezug zwischen der Personenanzahl der beiden Familien und der Aufteilung des Gewinns her.

\section{Transkript B2A1 - Can \& Philip - Szene 3 - Aufgabe 1}

\footnotetext{
Can: Guck mal Philip, Familie Schmidt hat 2 Personen, Familie Tenner hat 4 Personen.

Philip: (benutzt seinen Tintenkiller großflächig)

Can: Hä? Das war doch richtig.

Philip: Dann bekommen die 2 Sechstel und die 4 Sechstel.

beide: (Philip schreibt/zeichnet, can sieht ihm zu)

Can: (liest bei Philip:) 900 geteilt durch 6 sind 150 .

Philip: Und 2 Sechstel von 900 sind 300 . Ja.
} 
42

Can liest erneut die Aufgabenstellung durch und merkt an, dass Familie Schmidt aus zwei und Familie Tenner aus vier Personen besteht (34-35). Auf diese Bemerkung seines Partners löscht Philip aus, was er bisher notiert hat und erklärt ,dann bekommen die 2 Sechstel und die 4 Sechstel“ (38). Er notiert die Anteile und ergänzt in der Folge die vorgegebenen Pfeilschemata (Abb. 5.26). Während im ersten Pfeildiagramm zur Berechnung von $\frac{2}{6}$ von $900 €$ lediglich der Operator zum vervielfachen sowie das Ergebnis ergänzt werden müssen, sind im zweiten Pfeildiagramm keine Teillösungen vorgegeben. Auch hier trägt Philip die korrekten Rechenoperatoren und Ergebnisse ein: „900 geteilt durch 6 sind 150, mal 4 sind 600“ (44). Can übernimmt die Ergebnisse von Philip.

Die plötzliche Reaktion von Philip lässt vermuten, dass er die Information über die Anzahl der Familienmitglieder beim Lesen der Aufgabenstellung übersehen hat oder nicht für wichtig befunden hat. Als Can ihm die Information vorliest, reagiert Philip sofort, nennt die korrekten Anteile für die Familien und ergänzt die Berechnungen der Anteile im Pfeildiagramm. Er scheint unmittelbar mit dieser Information eine Beziehung zum Lösungsbeispiel herzustellen und den Lösungsweg zu übertragen.

Can stellt weiterhin keine Beziehung zum Lösungsbeispiel her, worauf seine Reaktion „Hä, das war doch richtig“ (37) auf Philips Auslöschen seiner bisherigen

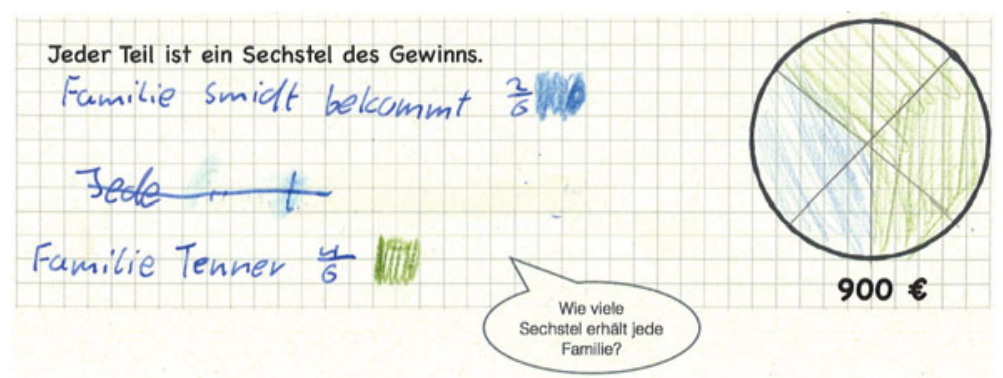

Abbildung 5.26 Philips Lösung von Aufgabenteil a) des ersten unvollständigen Beispiels 
Ergebnisse hindeutet. Im weiteren schreibt er die Lösungen seines Partners ab, ohne dass zu erkennen ist, dass er diese nachvollzieht oder reflektiert (Abb. 5.27).

b) Familie Schmidt: Familie Tenner:

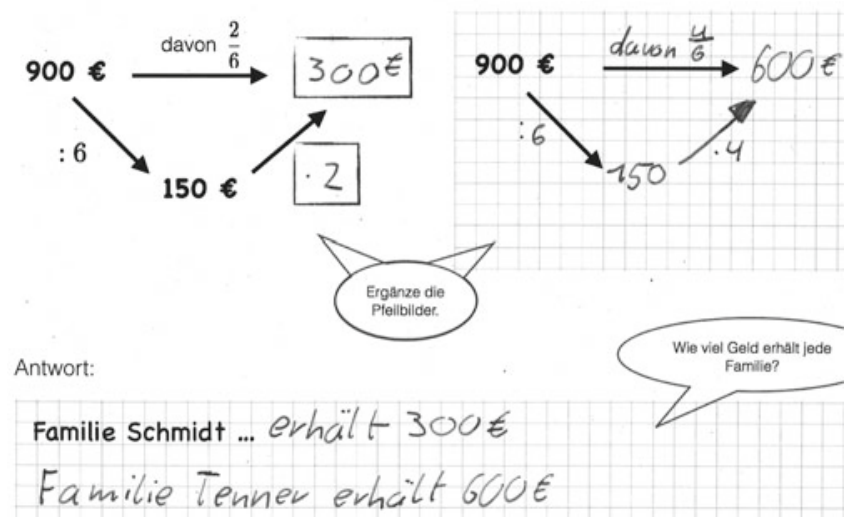

Abbildung 5.27 Philips Lösung von Aufgabe 1 b)

Insgesamt lässt sich die Bearbeitung von Can und Philip in folgenden Aspekten zusammenfassen:

- Can und Philip stellen keinen Bezug zur Situation in der Aufgabenstellung, dem Sachkontext im Allgemeinen oder dem Lösungsbeispiel her. Stattdessen versuchen sie die Aufgabe allein anhand der vorgegebenen Lösungsansätze und Zahlenwerte zu bearbeiten. Ihre Hauptschwierigkeit besteht darin, die Anteile zu bestimmen, zu denen der Geldbetrag auf die beiden Familien aufgeteilt werden soll. Zu einem Transfer des Vorgehens aus dem Lösungsbeispiel kommt es erst, als Can beim erneuten Lesen der Aufgabenstellung bemerkt, dass die beiden Familien aus unterschiedlich vielen Personen bestehen und die Personenzahlen nennt. Als unmittelbare Reaktion auf diese Information stellt Philip die Anteile für jede der beiden Familien fest und beginnt sofort die Geldbeträge zu berechnen. Es ist daher anzunehmen, dass die Schüler zu Beginn die Aufgabenstellung nicht eingehend genug gelesen haben und aufgrund des daraus resultierenden Mangels an Informationen versuchen die vorgegebenen Lösungsansätze zu vervollständigen, ohne einen wirklichen Bezug zur Aufgabenstellung oder eine Analogie zum Lösungsbeispiel herzustellen. 
- Unmittelbar nach der Feststellung der Anteile am Gewinn für jede Familie wendet Philip das Operatorschema zur Berechnung der Anteile an und ergänzt die fehlenden Angaben in den vorgegebenen Pfeildiagrammen. Dabei sind keine Schwierigkeiten erkennbar. Im Gegensatz zu Philip trägt sein Partner Can wenig zur Berechnung der Anteile bei und übernimmt zuletzt die Lösungen von seinem Partner. Insbesondere Cans als ,ratend“ zu charakterisierendes Vorgehen sowie sein kurzzeitiges beharren auf der Idee, dass beide Familien $\frac{3}{6}$ des Gewinns erhalten, bestärken die Annahme, dass er das Verfahren im Lösungsbeispiel nicht erfasst hat, sodass er entsprechend auch nicht auf dieses zurückgreifen kann.

\section{Transkript B2A2 - Can \& Philip - Szene 4 - Aufgabe 2}

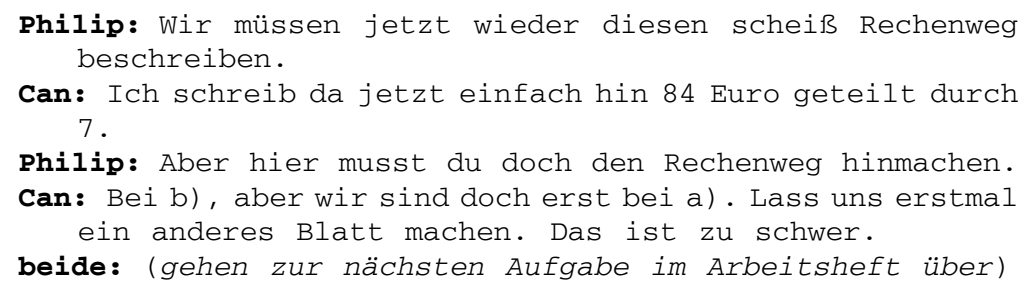

$\mathrm{Zu}$ Beginn der Bearbeitung des zweiten unvollständigen Beispiels lesen Can und Philip im Stillen die Aufgabenstellung. Philip erklärt umgehend, dass sie ,diesen [...] Rechenweg beschreiben“ (1-2) müssen, womit er sich vermutlich auf die Berechnung eines Anteils anhand des Operatorschemas bezieht. Sein Partner Can erwidert, dass er ,da jetzt einfach [...] 84 Euro geteilt durch 7“ (3-4) schreibe. Auf einen Einwand von Philip (5) erklärt er weiter, dass sie erst bei Teilaufgabe b) ,den Rechenweg“ aufschreiben müssen, sie jedoch erst bei Teilaufgabe a) seien (6-7). Es ist anzunehmen, dass er erkennt, dass seine Rechnung in den Aufgabenteil b) gehört, in dem die Anteile berechnet werden sollen, und er somit nicht weiß, was sie im Aufgabenteil a) machen sollen. Er stellt für sich fest ,das ist zu schwer“ (7) und schlägt vor, zunächst die übrigen Aufgaben im Arbeitsheft zu bearbeiten.

Es ist zu erkennen, dass Can nicht versucht den Aufgabenkontext zu erschließen und zur Erarbeitung eines Lösungsweg zu nutzen, sondern im Aufgabentext nach Zahlenwerten sucht, die er direkt in einer Rechnung zusammenstellen kann. Mit Ausnahme von Philips Feststellung, dass sie erneut eine Rechnung im Pfeilschema darstellen sollen, wird keine Beziehung zum Lösungsbeispiel oder dem ersten unvollständigen Beispiel hergestellt. Da die beiden Schüler auf Anhieb keinen Zugang zu der Aufgabe finden, beschließen sie, die Aufgabe an das Ende ihrer Bearbeitung des Arbeitshefts zurückzustellen, da sie ,zu schwer“ (7) sei. 


\section{Transkript B2A2 - Can \& Philip - Szene 5 - Aufgabe 2}

Am Ende der Unterrichtsstunde nehmen Can und Philip die Bearbeitung des zweiten unvollständigen Beispiels wieder auf. Nachdem Can seine Rechnung „,84 geteilt durch 7“ (9) vom ersten Bearbeitungsversuch wieder aufnimmt, fragt er, was sie machen sollen und bemängelt, dass man ihnen „,nicht erklärt“ (10) habe. Philip erwidert, dass „diese [...] Pfeilrechnung“ (11) überlegen müssen und das es „wie bei der ersten" (16) Aufgabe sei. Es wird nicht deutlich, ob er sich dabei auf das Lösungsbeispiel oder das erste unvollständige Beispiel bezieht. Es kann jedoch vermutet werden, dass er das Lösungsbeispiel meint, indem der Gewinn zunächst entsprechend der sieben Personen in sieben gleiche Teile geteilt wurde. In der Folge beginnt er ,84 geteilt durch 7“ (19) zu rechnen und kommt zu dem Ergebnis ,,das sind 12“ (20). Can stellt augenscheinlich einen Bezug zum Operatorschema her und 
fragt, was sie danach rechnen sollen: „Mal? 12 mal?“ (21). Diese Nachfrage deutet darauf hin, dass er das Operatorschema soweit verinnerlicht hat, dass er nach dem Teilen des Ganzen eine Multiplikation durchführen muss.

Philip ist ebenfalls irritiert und stellt fest, dass , da [...] kein Bruch [steht]“ (24). Er erkennt, dass er zum Berechnen eines Anteils zunächst wissen muss, welchen Anteil er berechnen soll. Entsprechend findet er keinen Faktor, mit dem er 12 multiplizieren kann. Er äußert, dass das Ergebnis „,einfach nur 12“ (24) sei, vermutet aber scheinbar, dass 12 nicht das korrekte Ergebnis ist.

Can nimmt entgegen Philips Unsicherheit das Ergebnis „12“ auf und folgert: „Lisa bekommt 12 Euro. Und dann bekommt er [Jan] den Rest“ (25). Beim Vergleich der entsprechenden Löhne für die beiden Jugendlichen bemerkt er dann aber auch, dass diese Annahme nicht stimmen kann, denn ,dann bekommt er doppelt so viel wie sie einfach" (26). Es ist bemerkenswert, dass er annimmt, dass die Differenz von 84 und 12 lediglich das Doppelte von 12 sei. Es ist jedoch möglich, dass er mit dem Doppelten meint, dass Jan in diesem Fall wesentlich mehr Geld bekäme als Lisa. Es ist jedoch nicht auszuschließen, dass er Schwierigkeiten beim Einordnen von natürlichen Zahlen hat.

Can wendet sich an das Paar, das am Nebentisch arbeitet, und merkt an, dass diese Aufgabe „voll schwer“ sei, da „kein Bruch“ angegeben sei (29-30) Aus diesem Grund wissen sie nicht, wie „man das rechnen“ (30) soll. Ihre Nachbarin erwidert, dass „die Stunden“ (31) der Bruch seien, womit sie meint, dass die Stunden die Grundlage zum Aufteilen des Ganzen sind. Can interpretiert diesen Hinweis jedoch wörtlich und versteht ihn dementsprechend nicht: ,7 Stunden ist der Bruch? Das geht nicht.“ (32).

Nach einer weiteren Ablenkung schlägt Can vor, dass sie den Gewinn einfach zu gleichen Teilen auf die beiden Jugendlichen aufteilen. Er sagt „,die Hälfte [von 84] ist 32" (36), was die Annahme seiner Unsicherheit beim Umgang mit natürlichen Zahlen stützt. Nach der Korrektur von Philip, dass die Hälfte von 84 nicht 32, sondern 42 sei, erklärt Can weiter: „42 Euro bekommt jeder“ (38). Philip lehnt diese Lösung jedoch ab (39).

Es ist deutlich zu erkennen, dass es Can und Philip nicht gelingt, die Anteile am Ganzen für beiden Jugendlichen zu bestimmen. Sie suchen weiterhin nach möglichen Kombinationen der Zahlen im Aufgabentext zur Durchführung einer Rechnung. Obwohl sie eine Ähnlichkeit zu den vorhergehenden Aufgaben und dem Lösungsbeispiel vermuten, stellen sie keine Beziehung zu diesen her. Dies kann möglicherweise dadurch erklärt werden, dass sie den Aufgabenkontext und die Aufgabensituation nicht in ihre Überlegungen einbeziehen, sondern lediglich nach Zahlenwerten zum Aufstellen einer Rechnung suchen. 


\section{Transkript B2A2 - Can \& Philip - Szene 6 - Aufgabe 2}

40 Nachbarin: Jan bekommt 5 Zwölftel.

41 Can: Was hast du gesagt? 5 Zwölftel bekommen die?

42 Nachbarin: Jan bekommt die.

43 Can: 5 Zwölftel ist richtig, weil 5 mal 12 sind 84 .

Ihre Tischnachbarin gibt ihnen einen zweiten, diesmal wesentlich expliziteren Hinweis und sagt ihnen vor, dass ,Jan [...] 5 Zwölftel [bekommt]“ (40). Can nimmt diesen Hinweis direkt auf und begründet ihn damit, dass ,,5 mal 12 [...] 84 [sind]“ (43). Er begründet demnach, dass $\frac{5}{12}$ ein korrekter Anteil sei, weil das Produkt von Zähler und Nenner die Ausgangsgröße ergebe. Ungeachtet des Rechenfehlers, der ihm hier unterläuft, deutet seine Begründung auf eine Fehlvorstellung hin: Er interpretiert, den Zähler und Nenner eines Bruchs nicht als Herstellungshandlungen oder 
Teiloperatoren, die auf eine Größe wirken, sondern als Faktoren, deren Produkt die Ausgangsgröße ergibt. Somit deutet der den Bruch $\frac{5}{12}$ weder als Anteil noch als Operator, sondern betrachtet den Bruch komponentenweise als Zusammenfassung zweier natürlicher Zahlen.

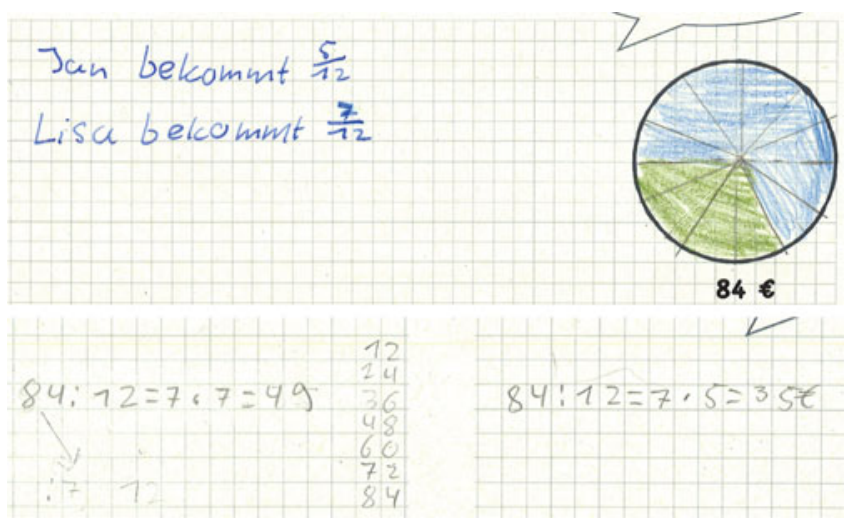

Abbildung 5.28 Philips Lösung von Aufgabe 2

Philip geht nicht auf Cans Erklärung ein, sondern schließt von dem Hinweis, dass Jan $\frac{5}{12}$ des Ganzen erhält, darauf dass Lisa $\frac{7}{12}$ des Ganzen bekommt (45). Es ist nicht zu entscheiden, ob er diesen Schluss zieht, da er den Zusammenhang zu den Arbeitsstunden erkennt, oder ob er lediglich den Bruch $\frac{5}{12}$ zu einem Ganzen ergänzt. Can stellt darauf hin den Zusammenhang zu den Anzahlen der geleisteten Arbeitsstunden her (46) und folgert für die ikonische Darstellung der Anteile im Kreisdiagramm: „Also müssen wir das in 12 Teile aufteilen, dieses Ding“ (47). In der Folge unterteilt Philip die vorgegebene Kreisfläche in zwölf gleiche Teile und färbt je acht und vier Teilsegmente in unterschiedlichen Farben als Repräsentation für die Arbeitsstunden der beiden Jugendlichen (vgl. Abb. 5.28). Die fehlerhafte Anzahl der gefärbten Teilsegmente kann dabei als Flüchtigkeitsfehler betrachtet werden. Can schaut Philip zunächst beim Zeichnen zu bevor er selber damit beginnt, die Anteile in der Kreisrepräsentation darzustellen. Er übernimmt die Zeichnung nicht von seinem Partner, sondern zeichnet die Anteile eigenständig ein (vgl. Abb. 5.29).

Nachdem beide Partner die Anteile eingezeichnet haben, gehen Can und Philip zur Berechnung der Anteile über. Philips Aussagen zeigen, dass er jetzt, wo er weiß, welche Anteile die beiden Jugendlichen jeweils bekommen, das Operatorschema anwendet, die jeweiligen Teiloperatoren $: 12$ und .5 bzw. .7 korrekt bestimmt und die Anteile berechnet. 


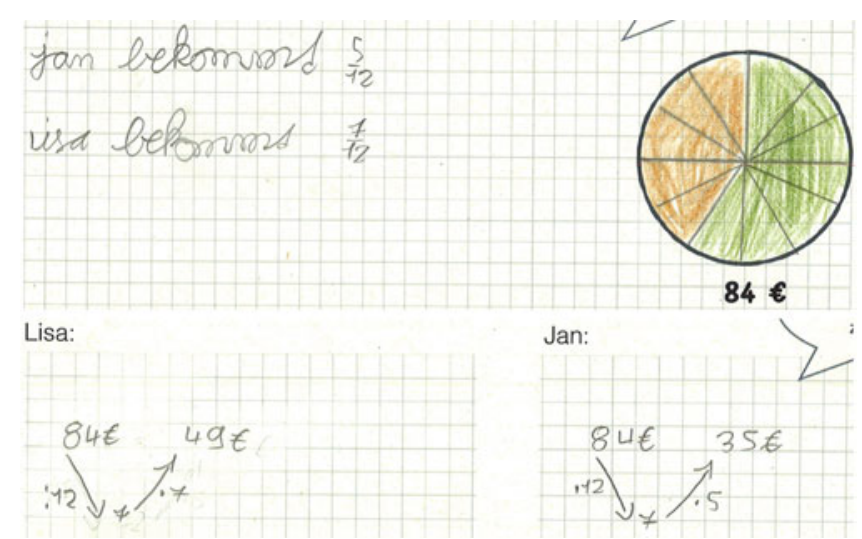

Abbildung 5.29 Can Lösung von Aufgabe 2

Im Gegensatz zu Philip zeigt Can Unsicherheiten bezüglich der Berechnung der Anteile. Er fragt seinen Partner zunächst wiederholt, wie viel $\frac{5}{12}$ von 84 sind, wie viel Geld jeder bekommt (51-53) und was Philip macht, um die Anteile zu berechnen (56). Philip erklärt ihm den Rechenweg und Can notiert ihn in seinem Arbeitsheft. Obgleich die Interaktion und Cans wiederholte Blicke auf Philips Arbeitsheft den Anschein erwecken, dass er die Rechnungen bei seinem Partner abschreibt, sind deutliche Unterschiede in der Notation der beiden Schüler zu erkennen. Während Philip die Rechnungen in einer Art Term notiert, in dem er schreibt $84: 12=7 \cdot 7=49$ (vgl. Abb. 5.28), notiert Can den Rechenweg korrekt in einem Pfeilschema (vgl. Abb. 5.29). Es ist anzunehmen, dass er aufgrund großer Unsicherheit sich mehrfach bei seinem Partner versichert, wie der Rechenweg lauten muss, jedoch die Darstellung der Rechnung in einem Pfeilschema übertragen und in dieser Situation anwenden kann. Es kann daher angenommen werden, dass er die Struktur der Rechnung im Allgemeinen überträgt, jedoch Schwierigkeiten hat, die korrekten Rechenoperatoren eigenständig herzuleiten. Diese Interpretation wird durch die beobachteten Schwierigkeiten Cans beim Rechnen mit natürlichen Zahlen sowie seine vorhergehende Begründung, dass $\frac{5}{7}$ der korrekte Anteil sei, da $5 \cdot 7=84$ sei, gestützt.

Zuletzt äußert Can seine Irritation darüber, dass Lisa mehr Geld ausgezahlt bekommt als Jan. Auch die Differenz der Geldbeträge scheint er nicht korrekt zu erfassen, da er anmerkt, dass Lisa lediglich ,zwei Stunden“ mehr gearbeitet habe und mit $49 €$ „direkt das doppelte“ (76) von $35 €$ bekomme. Aufgrund dieser Äußerungen von Can ist zu anzunehmen, dass er die Anteilbildung in dieser Situation 
noch nicht vollständig verstanden hat. Ihm scheint nicht ersichtlich zu sein, dass die beiden Jugendlichen entsprechend ihrer unterschiedlichen Anzahl an geleisteten Arbeitsstunden einen unterschiedlichen Betrag ausgezahlt bekommen. Obwohl seine Irritation jedoch auch mit der weit überschätzten Differenz der Geldbeträge erklärt werden kann, ist vor dem Hintergrund seiner vorhergehenden Aussagen, geäußerten Verständnisschwierigkeiten und Fehlinterpretationen von Anteilen dennoch anzunehmen, dass er den Bezug der Anteile zur Aufgabensituation nicht vollständig hergestellt und verstanden hat, auch wenn er den Hinweis der Sitznachbarin wiederholt, dass die Anteile der beiden Jugendlichen etwas damit zu tun haben, dass Lisa 7 und Jan 5 Stunden gearbeitet hat (46-47).

Insgesamt lässt sich die Bearbeitung von Can und Philip in den folgenden Deutungshypothesen zusammenfassen:

- Es wird kein Bezug zum Lösungsbeispiel oder dem ersten unvollständigen Beispiel hergestellt und demzufolge auch keine Analogie zum Transfer des Lösungsverfahrens gebildet. Im Lösungsbeispiel sowie im ersten unvollständigen Beispiel werden die Anteile des Ganzen gemäß der Personenanzahl gebildet. Im zweiten unvollständigen Beispiel hingegen müssen die Anteile entsprechend der Arbeitszeit in Stunden gebildet werden. Can und Philip identifizieren zwar den Geldbetrag von $84 €$ als das Ganze, jedoch gelingt es ihnen nicht die Anteile zu bestimmen, auf die das Ganze aufgeteilt werden soll. Es anzunehmen, dass der ausbleibende Transfer auf die veränderten Kontextbedingungen im Vergleich zum Lösungsbeispiel und dem ersten unvollständigen Beispiel zurückzuführen ist. Entsprechend der Aufteilung auf Grundlage der Anzahl an Personen wird eine Aufteilung in gleiche Teile vorgeschlagen, jedoch aufgrund der unterschiedlichen Anzahl an Arbeitsstunden der beiden Jugendlichen verworfen. Eine Aufteilung anhand der Arbeitsstunden wird dennoch nicht in Betracht gezogen.

Auch mit dem Hinweis ihrer Sitznachbarin, dass sie die Anteile entsprechend der Arbeitszeit in Stunden bilden sollen, gelingt es ihnen nicht die Anteile zur Aufteilung des Ganzen zu bestimmen. Der Grund dafür ist, dass sie lediglich den Geldbetrag von $84 €$ als Ganzes identifizieren und nicht erkennen, dass dieser Geldbetrag mit der Summe der Arbeitsstunden im Zusammenhang steht.

- Die Schwierigkeit die Anteile am Gewinn zu bestimmen führt dazu, dass Can und Philip versuchen anhand der Zahlenwerte im Aufgabentext eine Rechnung zusammenzustellen, ohne dabei den Aufgabenkontext einzubeziehen.

- Ungeachtet ihrer Schwierigkeiten bei der Bestimmung der Anteile übertragen Can und Philip das Operatorschema zur Berechnung der Anteile. Nachdem ihre Sitznachbarin ihnen gesagt hat, welche Anteile die beiden Jugendlichen erhalten, beginnen Can und Philip sofort damit die Anteile auszurechnen. Während Can 
zunächst Unsicherheiten bezüglich der Teiloperatoren und Zwischenergebnisse zeigt, wendet Philip das Operatorschema sicher an. Im Gegensatz zu Can, der den Rechenweg in einem Pfeildiagramm darstellt, notiert Philip eine Art Term, in dem er die beiden Teilrechnungen hintereinander ausführt.

- Can deutet den Bruch im Anteil $\frac{5}{12}$ von $84 €$ im Sinne einer komponentenweise Betrachtung von Zähler und Nenner und argumentiert, dass 5·12=84 sei. Diese Fehlinterpretation kann einerseits auf die fehlerhafte Kopfrechnung zurückgeführt werden, deutet andererseits aber auch darauf hin, dass er den Bruch in diesem Zusammenhang weder als Anteil noch als Operator, sondern als zwei natürliche Zahlen betrachtet, was möglicherweise zur Entwicklung eines fehlerhaften Denkmusters bzw. einer Fehlvorstellung führen kann.

\section{Vergleich der Bearbeitungen der unvollständigen Beispiele:}

Die Bearbeitung erfordert die Übertragung des Verfahrens der Anteilbestimmung und Anteilberechnung von dem Lösungsbeispiel auf nahezu identische Aufgabenstellung mit veränderten Zahlenwerten sowie auf eine analoge Aufgabenstellung in einem neuen strukturgleichen Sachkontext mit anderen Bezugsgrößen. Die zentralen Aspekte in den Detailanalysen der Bearbeitungsprozesse waren dabei zum einen die Bildung einer Analogie zwischen dem Lösungsbeispiel und den unvollständigen Beispielen sowie die Übertragung des Operatorschemas zur Bestimmung und Berechnung von Anteilen eines Ganzen.

Analogiebildung: In den beiden dargestellten Bearbeitungsprozessen können die zentralen Schwierigkeiten der Aufgabenlösung im Rahmen der Analogiebildung als Voraussetzung zur Übertragung des Lösungsverfahrens beschrieben werden. Anders als es aufgrund der sachanalytischen Analysen erwartet wurde, betrifft dies bereits die Bearbeitung des ersten unvollständigen Beispiels, in dem sowohl der identische Sachkontext sowie die beinhalteten Größen eine direkte Abbildung der Lösungsschritte ermöglichen sollten.

Bennet und Julius erkennen zunächst keinen Zusammenhang zu dem Lösungsbeispiel und betrachten das erste unvollständige Beispiel als vollkommen neue Aufgabe. Julius bemerkt gar, dass es für diese Aufgabe kein Lösungsbeispiel gebe. Erst im Austausch mit der Lehrkraft, die sie zunächst auf die Beziehung mit dem Lösungsbeispiel hinweist und zudem die Aufmerksamkeit der Schüler auf den Kontext der Aufgabe, insbesondere das Aufteilen eines Geldbetrags auf zwei Familien mit unterschiedlichen vielen Familienmitgliedern lenkt, erkennen sie die analoge Struktur zum Lösungsbeispiel. In der Folge bilden sie erfolgreich und ohne erkennbare inhaltliche Verständnisschwierigkeiten die einzelnen Verfahrensschritte auf die neuen Zahlenwerte ab. 
Genau wie Bennet und Julius erkennen auch Can und Philip zunächst keinen Zusammenhang zwischen dem Lösungsbeispiel und dem ersten unvollständigen Beispiel. Es fällt auf, dass der Sachkontext in ihre Bearbeitung zunächst nicht aufgegriffen wird und die Schüler versuchen allein auf Grundlage der vorgegebenen Lösungsansätze und Zahlenwerte die Aufgabe zu bearbeiten. Dies scheitert zunächst daran, dass es ihnen nicht gelingt die Gewinnanteile für jede Familie zu bestimmen, die genau wie im Lösungsbeispiel von der Anzahl der Familienmitglieder der beiden Familien abhängt. Erst als Can scheinbar zufällig beim wiederholten Lesen der Aufgabenstellung bemerkt, dass die beiden Familien aus unterschiedlichen vielen Personen bestehen, stellt Philip erkennt Philip den Zusammenhang zum Lösungsbeispiel und es gelingt ihm die einzelnen Lösungsschritte zu übertragen.

Beide Bearbeitungsprozesse haben gemeinsam, dass die Schüler ihre Aufmerksamkeit zunächst nicht auf die Anzahl der Personen in jeder Familie richten und entsprechend keine Beziehung zum Lösungsbeispiel herstellen, obwohl der Lösungsweg bereits vorstrukturiert und mit Hinweisen versehen ist. Die Bearbeitungsprozesse beider Schülerpaare deuten darauf hin, dass die vom Lösungsbeispiel verschiedene äußere Gestaltung des ersten unvollständigen Beispiels ein wesentlicher Grund für das anfängliche Nichterkennen der Strukturgleichheit zwischen diesen beiden ist. Während im Lösungsbeispiel die Aufteilung des Ganzen auf die jeweiligen Familien und Familienmitglieder in Form von ikonischen Personendarstellungen und einer ikonischen Kreisrepräsentation dynamisch veranschaulicht wird, sind im ersten unvollständigen Beispiel keine Abbildungen von Personen enthalten. Stattdessen ist die Darstellung der Aufgabenstellung auf einen Text beschränkt, der die Anzahl der Familienmitglieder in Klammern hinter den Familiennamen angibt. Auch die vorgegebene Kreisrepräsentation enthält keinen Hinweis auf die Anteile für die beiden Familie, da diese von den Schülern selbst eingezeichnet werden müssen. Aus diesen Gründen wird die Aufmerksamkeit nicht direkt auf die Personen gelenkt und die Schüler suchen nach alternativen Möglichkeiten der Anteilbestimmung. Dies wird insbesondere durch die Vorgabe von Lösungsansätzen unterstützt, da diese nahelegt, das die Lösungsschritte nur ergänzt werden müssen. Vor allem in der Bearbeitung von Can und Philip ist zu beobachten, dass die Schüler zunächst ausschließlich in den Lösungsansätzen nach Hinweisen für die Anteilbestimmung suchen, und den Sachkontext im Aufgabentext nicht beachten.

In den Bearbeitungen des zweiten unvollständigen Beispiels besteht die Bildung einer Analogie in der Abbildung der Strukturelemente unter Beachtung der unterschiedlichen Bezugsgrößen (vgl. Tab. 5.3). 
Tabelle 5.3 Abbildung der Kontextelemente des Lösungsbeispiels auf das zweite unvollständige Beispiel

\begin{tabular}{l|l}
\hline Lösungsbeispiel & Zweites unvollständiges Beispiel \\
\hline Das Ganze ist ein Geldbetrag von 21000€. & Das Ganze ist ein Geldbetrag von 84€. \\
\hline $\begin{array}{l}\text { Der Geldbetrag wird auf die zwei Familien } \\
\text { Meyer und Stein aufgeteilt. }\end{array}$ & $\begin{array}{l}\text { Der Geldbetrag wird auf die zwei } \\
\text { Jugendlichen Lisa und Jan aufgeteilt. }\end{array}$ \\
\hline $\begin{array}{l}\text { Die Familien haben eine unterschiedliche } \\
\text { Anzahl an Familienmitgliedern. Familie } \\
\text { Meyer besteht aus drei Personen, Familie }\end{array}$ & $\begin{array}{l}\text { Die beiden Jugendlichen haben eine } \\
\text { unterschiedliche Anzahl an Stunden } \\
\text { gearbeitet. Lisa hat sieben Stunden } \\
\text { gearbeitet und Jan fünf. }\end{array}$ \\
\hline $\begin{array}{l}\text { Insgesamt wird der Geldbetrag auf sieben } \\
\text { Personen aufgeteilt. }\end{array}$ & $\begin{array}{l}\text { Die beiden Jugendlichen haben zusammen } \\
\text { zwölf Stunden gearbeitet. }\end{array}$ \\
\hline $\begin{array}{l}\text { Jede Familie erhält jeweils einen Teil für } \\
\text { jedes Familienmitglied. }\end{array}$ & $\begin{array}{l}\text { Lisa und Jan erhalten jeweils einen Teil für } \\
\text { jede Arbeitsstunde. }\end{array}$ \\
\hline
\end{tabular}

Im Fall von Bennet und Julius konnte beobachtet werde, dass Bennet quasi spontan die in Tabelle 5.3 beschriebenen Abbildungen vorgenommen hat, und auf diese Weise die neue Sachsituation erfassen und erschließen konnte, sodass er sofort erklärt, dass das Ganze in zwölf gleiche Teile aufgeteilt werden muss, die dann auf die beiden Jugendlichen aufgeteilt werden. Seinem Partner Julius gelingt die Abbildung nicht auf Anhieb, sondern erst durch einen Hinweis von Bennet. Julius geäußerte Verständnisschwierigkeiten bringen zum Ausdruck, dass es in seinem Fall womöglich nicht allein die veränderten Bezugsgrößen sind, die ihn irritieren. Seine Irritation betrifft vor allem die Beschreibung der Sachsituation, dass zwei Jugendliche unterschiedlich lange gearbeitet haben und gemeinsam einen Lohn für ihre Arbeit ausgezahlt bekommen. Für gewöhnlich bekommt man den Lohn für seine Arbeit direkt ausgezahlt und muss ihn sich nicht mit einem Arbeitskollegen teilen. Sein erster Vorschlag für eine Aufteilung ist, dass sie den Lohn in gleiche Teile aufteilen. Erst als Bennet ihn darauf hinweist, dass die beiden Jugendlichen unterschiedlich lange gearbeitet haben, erkennt er, dass der Lohn durch die Summe der Arbeitsstunden in zwölf gleiche Teile aufgeteilt werden muss, von denen Lisa und Jan schließlich die ihren Arbeitsstunden entsprechende Anzahl an Teilen bekommen.

Can und Philip haben wesentlich größere Schwierigkeiten mit der Abbildung der strukturrelevanten Aufgabenelemente. Sie identifizieren den Geldbetrag von $84 €$ sofort als Ganzes und erkennen auch, dass dieser gerecht auf die zwei Jugendlichen aufgeteilt werden soll. Sie stellen jedoch keinen Zusammenhang zwischen der Anzahl der Arbeitsstunden der beiden Jugendlichen und der Aufteilung des Ganzen 
her und es gelingt ihnen aufgrund dessen nicht, die Anteile am Geldbetrag für die beiden Jugendlichen zu bestimmen. In ihren Bearbeitungsvorschlägen wird deutlich, dass sie nicht versuchen auf Grundlage des Sachkontexts die Anteile abzuleiten, sondern versuchen anhand der enthaltenen Zahlenwerte direkt die Geldbeträge zu berechnen. Auch ein erster Hinweis ihrer Sitznachbarin, dass die Stunden zur Anteilbildung genutzt werden sollen, hilft ihnen nicht. Erst als ihre Nachbarin expliziter wird und ihnen den Anteil nennt, den Jan bekommt, erschließen sie auch den Anteil für Lisa und stellen die Anteile im Kreisdiagramm dar. Diese Beobachtungen legen den Schluss nahe, dass Can und Philip zwar die Stundenanzahl wahrgenommen haben, jedoch nicht den Zusammenhang entdeckt haben, dass der Geldbetrag der Summe der Arbeitsstunden entspricht. Es ist möglich, dass ihre Schwierigkeiten bei der Analogiebildung auf ihr flüchtiges Lesen des Lösungsbeispiels zurückzuführen ist. Anstatt die einzelnen Lösungsschritte nachzuvollziehen, haben sie diese lediglich schnell durchgeklickt und die Antworten auf die fokussierenden Fragestellungen in den Endzuständen des Lösungsbeispiels gesucht. Diese Beobachtung stützt die Vermutung einer oberflächlichen Verarbeitung des Lösungsbeispiels, die dazu führt, dass sie die strukturellen Zusammenhänge nicht vollständig erschließen und entsprechend auf die Anteilbildung im zweiten unvollständigen Beispiel übertragen können.

Zusammenfassend kann angenommen werden, dass die folgenden Aspekte den Transfer des Lösungswegs auf die unvollständigen Beispiele beeinflussen:

- Die äußere Gestaltung der unvollständigen Beispiele,

- Oberflächliches Lesen des Lösungsbeispiels,

- geringe Vertrautheit mit der Sachsituation,

- die Fokussierung auf den Rechenweg,

- Irritationen durch veränderte Größen und Bezüge zueinander.

Übertragung und Anwendung des Operatorschemas: Im Gegensatz zum Bilden der Anteile des Ganzen gelingt es beiden Schülerpaaren das Operatorschema zur Berechnung der Anteile auf die unvollständigen Beispiele zu übertragen und anzuwenden. Im Fall von Julius ist zu beobachten, dass er bei der Bearbeitung des ersten unvollständigen Beispiels noch deutliche Unsicherheiten bei der Bestimmung der Teiloperatoren hat, und nicht sicher ist, auf welche Größen diese angewendet werden. In der Bearbeitung des zweiten unvollständigen Beispiels sind diese Unsicherheiten hingegen nicht mehr zu beobachten. Diese Beobachtung kann als eine zunehmende Vertrautheit und Verinnerlichung mit dem Verfahren interpretiert werden. 
Ähnliche Unsicherheiten, wie sie in Julius Bearbeitung des ersten unvollständigen Beispiels zu beobachten sind, können auch im Fall von Can gemacht werden. Er versichert sich sowohl im ersten wie auch im zweiten unvollständigen Beispiel bei seinem Partner über die korrekten Rechenschritte.

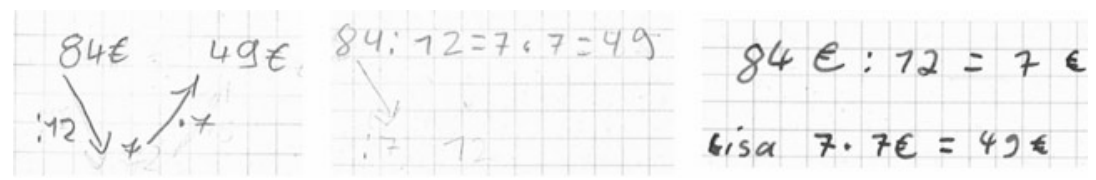

Abbildung 5.30 Cans, Philips und Julius (von links nach rechts) Notation der Berechnung von $\frac{7}{12}$ von 84

In Bezug auf die Notation des Rechenwegs ist festzustellen, dass lediglich Can die Darstellung der Rechnung im zweiten unvollständigen Beispiel anhand eines Pfeildiagramms vornimmt. Die anderen Schüler notieren den Rechenweg in Form von Termen (vgl. Abb. 5.30). Insbesondere in der Abbildung der Rechnung von Philip ist zu erkennen, dass er zunächst $84: 7$ gerechnet hat, und diesen Rechenweg in einem Pfeildiagramm darstellen wollte. Da er diese Rechnung im Rahmen der Suche nach einem Lösungsweg aufgestellt hat, ist anzunehmen, dass er sich hierbei nicht sicher ist ob diese Rechnung zum Ziel führt. Zusammen mit der Beobachtung von Cans Unsicherheiten bei der Berechnung der Anteile kann vermutet werden, dass die Schüler im Fall von Unsicherheit die Darstellung in einem Pfeildiagramm bevorzugen bzw. sich an diesem orientieren. In Bennet und Julius sowie später auch Philips Anteilberechnung im zweiten unvollständigen Beispiel sind keine Unsicherheiten oder Schwierigkeiten zu erkennen und sie notieren den Rechenweg ohne die Darstellung in einem Pfeildiagramm in einer ihnen womöglich vertrauteren Termschreibweise. In diesem Zusammenhang merkt Bennet an, dass er die Rechnung an sich ,einfach“ findet, jedoch die Darstellung im Pfeildiagramm einen zusätzlichen und womöglich unnötigen Aufwand darstellt.

\subsubsection{Anwendung des Verfahrens in einem komplexen Sachkontext}

Im Anschluss an die Bearbeitung der unvollständigen Beispiele haben die Schülerinnen und Schüler in ihren Arbeitsheften verschiedene Aufgaben zum Berechnen von Anteilen beliebiger Größen bearbeitet. Diese umfassten u. a. Aufgaben zur Berech- 
nung von Anteilen von Strecken und Gewichten, Anteilen von mehreren Ganzen sowie Aufgaben, in denen Fehler in vorgegebenen Rechnungen gesucht und korrigiert werden sollten. Die nun vorliegende Aufgabe geht über die Anwendung des Verfahrens zur Berechnung von Anteilen beliebiger Größen hinaus, da nicht wie bisher ein Anteil von einer beliebigen Größe berechnet, sondern von einem Anteil einer beliebigen Größe auf einen anderen Anteil derselben Größe geschlossen werden soll (Abb. 5.31).

\section{Aufgabe 9}

Bei einer Fahrradkontrolle wurden an 34 Fahrrädern Mängel festgestellt. Das waren $\frac{2}{7}$ aller kontrollierten Fahrräder.

An wie vielen Fahrrädern wurden keine Mängel festgestellt?

\section{Abbildung 5.31 Aufgabe 2.9}

Im Aufgabentext ist die Anzahl von 34 Fahrrädern gegeben, die $\frac{2}{7}$ der Gesamtmenge kontrollierter Fahrräder entspricht. Es soll berechnet werden, an wie vielen Fahrrädern keine Mängel festgestellt wurden. Dazu ist es notwendig die 34 Fahrräder als Teil eines Ganzen zu interpretieren und einen Bezug zum Ganzen bzw. der Anzahl aller kontrollierten Fahrräder herzustellen. Es sind drei Lösungswege denkbar:

1. Bestimmen des Ganzen und Berechnen von $\frac{5}{7}$ des Ganzen als komplementären Anteil zu $\frac{2}{7}$ (Zweisatz):

34 Fahrräder entsprechen $\frac{2}{7}$ aller kontrollierten Fahrräder. Zur Bestimmung des Ganzen muss somit das Verfahrens zur Anteilberechnung umgekehrt werden:

34 Fahrräder : $2=17$ Fahrräder und 17 Fahrräder $\cdot 7=119$ Fahrräder.

Es wurden also insgesamt 119 Fahrräder kontrolliert. Da an $\frac{2}{7}$ dieser Fahrräder Mängel festgestellt wurden, wurden an $\frac{5}{7}$ von 119 Fahrrädern keine Mängel festgestellt:

119 Fahrräder : $7=17$ Fahrräder und 17 Fahrräder $\cdot 5=85$ Fahrräder.

2. Bestimmen von $\frac{1}{7}$ des Ganzen und Berechnen von $\frac{5}{7}$ des Ganzen (Anwenden von Operatoren):

Da 34 Fahrräder $\frac{2}{7}$ aller kontrollierten Fahrräder sind, entspricht die Hälfte dieser 34 Fahrräder, also 17 Fahrräder, $\frac{1}{7}$ aller kontrollierten Fahrräder. Durch Multiplikation mit 5 erhält man $\frac{5}{7}$ des Ganzen bzw. die Anzahl der Fahrräder, an denen 
keine Mängel festgestellt wurden:

34 Fahrräder : $2=17$ Fahrräder und 17 Fahrräder $\cdot 5=85$ Fahrräder.

3. Bestimmen des Ganzen und Bilden der Differenz:

34 Fahrräder entsprechen $\frac{2}{7}$ aller kontrollierten Fahrräder. Zur Bestimmung des Ganzen muss somit das Verfahrens zur Anteilberechnung umgekehrt werden: 34 Fahrräder : $2=17$ Fahrräder und 17 Fahrräder $\cdot 7=119$ Fahrräder. Da an 34 von 119 Fahrrädern Mängel festgestellt wurden, sind an $119-34=85$ Fahrrädern keine Mängel festgestellt worden.

Transferprozesse: Die Lösung dieser mehrschrittigen Aufgabe erfordert sowohl die Aktivierung der Anteilvorstellung als auch der Operatorvorstellung. Im ersten Schritt muss der Bruch $\frac{2}{7}$ als Anteil interpretiert werden, um abzuleiten, dass zu einem Ganzen noch $\frac{5}{7}$ fehlen. Auf Grundlage der Operatorvorstellung muss in der Folge entweder das Verfahren zur Anteilberechnung rückgängig gemacht werden, um vom Anteil ausgehend das Ganze zu berechnen, oder von $\frac{2}{7}$ auf $\frac{1}{7} \mathrm{zu}$ schließen, um $\frac{5}{7}$ berechnen zu können.

Somit besteht der Transfer in dieser Aufgabenstellung zum einen in der Interpretation der Sachsituation auf Grundlage der Anteilvorstellung zum Bestimmen des Anteils, eines Teils und des Ganzen sowie zum Herstellen eines Zusammenhangs zwischen diesen. Zum anderen ist es erforderlich das Operatorschema bzw. das Verfahren zur Berechnung von Anteilen zu übertragen und anzuwenden, um ausgehend von dem vorgegebenen Anteil des Ganzen einen Teil des Ganzen zu berechnen und mit diesem das Ganze oder den gesuchten Anteil vom Ganzen zu berechnen. Die Anwendung des Operatorschemas erfordert zudem eine Anpassung in Form der Umkehrung des Verfahrens zum Rückgängigmachen von Teiloperationen der Anteilberechnung.

Auf Ebene des Sachkontexts besteht der Transfer in dieser Aufgabe zudem in der Übertragung der Anwendung des Verfahrens von standardisierten Maßeinheiten (Geldbeträge in $€$, Längen, Gewichten) als Größe auf eine diskrete Menge von Objekten (Fahrräder).

\section{Bennet \& Julius - Bestimmen des Ganzen}

Im Anschluss an die Bearbeitung der unvollständigen Beispiele haben Bennet und Julius die nachfolgenden Aufgaben im Arbeitsheft der Reihe nach gelöst. Dabei konnten keine besonderen Schwierigkeiten beobachtet werden. Einzig in der Bearbeitung einer Aufgabe zum Bestimmen eines Anteils mehrerer Ganzer, in der gefragt wurde, wie viel Pizza jede Person bekommt, wenn sich sechs Personen 4 Pizzas teilen, konnte beobachtet werden, dass Julius einen zeichnerischen Lösungsansatz 
verfolgte. Bennet hingegen interpretierte die Situation im Rahmen einer Division und beschrieb die Aufteilung auf symbolischer Ebene als „,4 Pizzen : $6=\frac{4}{6}$ Pizzen“. Während Bennet bereits zur nächsten Aufgabe übergehen wollte, war Julius noch mit dem Zeichnen von vier Kreisen beschäftigt, was er kurzerhand abbrach und die Lösung von seinem Partner übernahm. Die unterschiedlichen Lösungsansätze in dieser Aufgabe sind charakteristisch für die allgemeinen Zugänge von Bennet und Julius: Während Julius stets versucht, die Aufgaben auf eine anschauliche Ebene zu übertragen, arbeitet Bennet stets auf rein symbolischer Ebene und fertigt nur Darstellungen an, wenn es in der Aufgabenstellung vorgesehen ist.

\section{Transkript B2A9 - Bennet \& Julius - Szene 1 - Aufgabe 9}

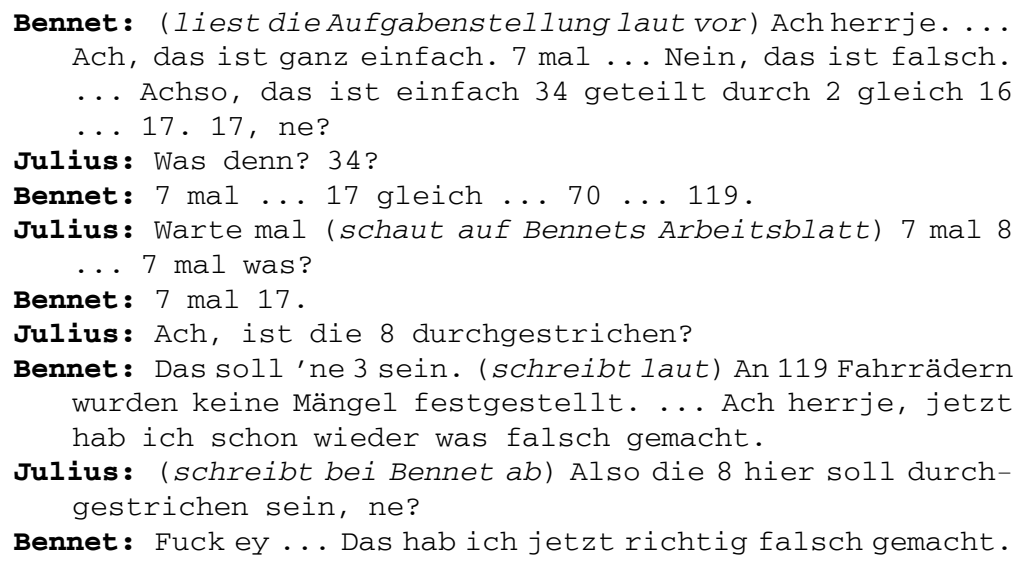

Direkt nach dem Lesen der Aufgabenstellung erweckt Bennet den Eindruck, dass er mehrere Ideen für einen Lösungsweg hat. Zunächst scheint er die Aufgabe schwierig zu finden, kurz darauf wiederum ,ganz einfach“ (1-2). Sein erster Lösungsvorschlag ist die Berechnung des Ganzen durch eine Multiplikation mit sieben (2). Diesen Vorschlag revidiert er kurz darauf jedoch wieder und erklärt, dass man zunächst ,„34 geteilt durch 2" (3) rechnen müsse.

Intuitiv nimmt er zunächst an, dass er über eine Multiplikation mit sieben das Ganze erhält (2), was er nach kurzem Nachdenken jedoch revidiert und erklärt, dass man zunächst ,34 geteilt durch 2“ (3) rechnen müsse. Er kommt zu dem Zwischenergebnis 17, multipliziert mit 7 und nennt 119 als Ergebnis (6). In seinem Arbeitsheft notiert er laut mitlesend: „An 119 Fahrrädern wurden keine Mängel festgestellt" (11-12). Unmittelbar nachdem er seinen Satz beendet hat bemerkt er jedoch, dass er „schon wieder was falsch gemacht“ $(12-13,16)$ habe. 
Bennets Erklärungen bieten Anlass zur Annahme, dass er den Lösungsweg nach und nach erschließt, indem er eine Idee ausprobiert, diese reflektiert und korrigiert. Auf diese Weise folgt er zunächst seiner ersten Idee, die vorgegebene Anzahl von 34 Fahrrädern mit sieben zu multiplizieren, um das Ganze bzw. die Anzahl aller kontrollierten Fahrräder zu berechnen. Er bemerkt jedoch, dass die Anzahl 34 nicht $\frac{1}{7}$, sondern $\frac{2}{7}$ des Ganzen sind und korrigiert seine Rechnung umgehend. Er dividiert im Weiteren durch 2 und multipliziert anschließend mit 7. Während er seine Lösung notiert, bemerkt er seinen Fehler, dass er lediglich das Ganze bestimmt hat, was jedoch nicht der Anzahl aller Fahrräder ohne Mängel entspricht.

Julius trägt zu der Bearbeitung nichts bei. Er fragt wiederholt, was Bennet rechnet und aufschreibt, stellt jedoch keine inhaltlichen Nachfragen oder beteiligt sich konstruktiv an der Lösung.

\section{Transkript B2A9 - Bennet \& Julius - Szene 2 - Aufgabe 9}

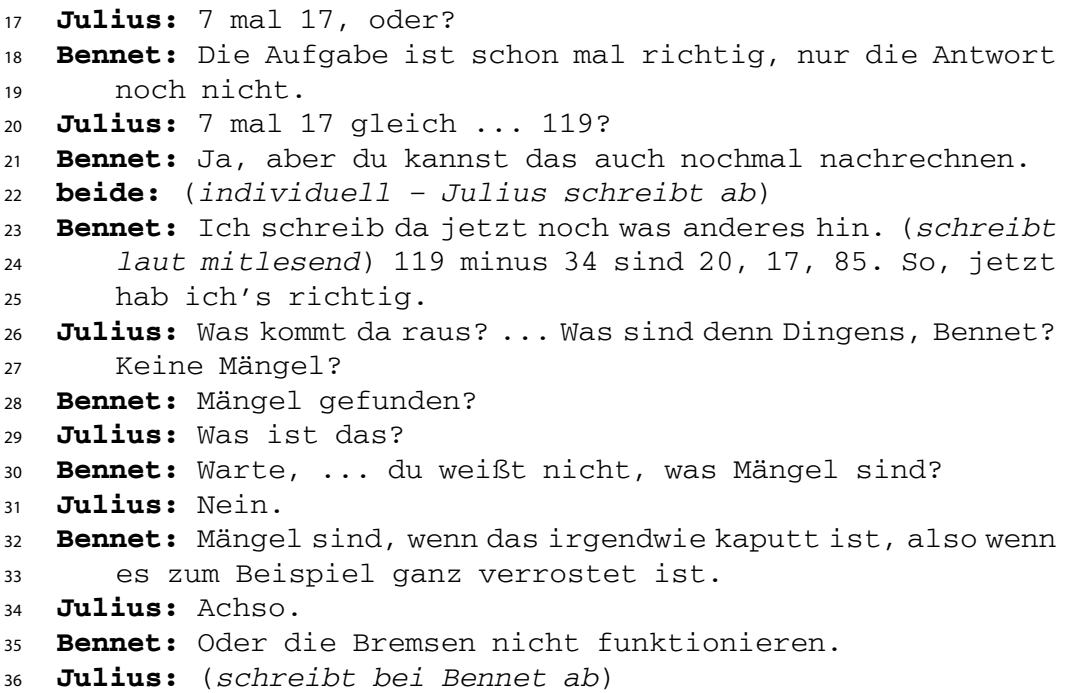

Bennet erklärt, dass seine Rechnung bisher „,schon mal richtig“ sei, „nur die Antwort noch nicht" (18-19). Wenig später er notiert er erneut laut mitlesend seine Lösung und stellt fest: „So, jetzt hab ich's richtig“ (23-25). Da er bereits die Anzahl aller kontrollierten Fahrräder berechnet hat, subtrahiert er von diesen die Anzahl der mangelhaften Fahrräder und kommt zu dem Ergebnis, dass 85 Fahrräder die Kontrolle ohne Mängel passiert haben. In seinem Lösungsweg (Abb. 5.32) ist deutlich 
zu erkennen, dass er sich Schritt für Schritt dem einem richtigen Lösungsweg annähert, sein Vorgehen dabei stets hinterfragt und in Beziehung zum Aufgabenkontext setzt. Dabei wird deutlich, dass er das Operatorschema äußerst flexibel anwendet und problemlos Beziehungen zwischen Teil und Ganzem herstellt und diese rechnerisch bestimmen kann. Obgleich er zunächst die 34 Fahrräder als $\frac{1}{7}$ interpretiert, bemerkt er seinen Fehler und korrigiert diesen umgehend.

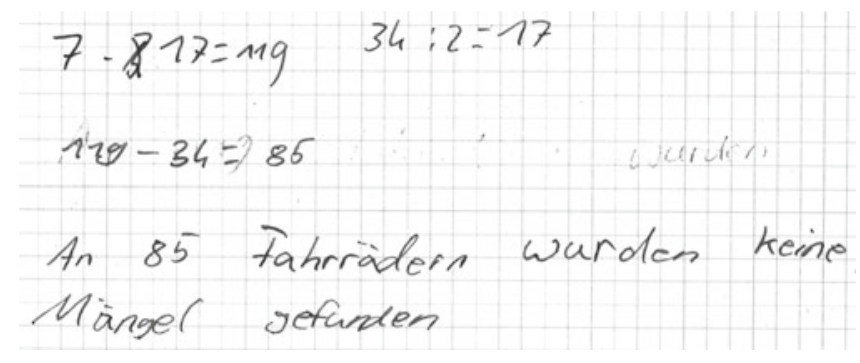

Abbildung 5.32 Bennets Lösung von Aufgabe 2.9

Seine Erklärungen deuten darauf hin, dass er die Aufgabensituation zunächst nicht richtig gelesen oder verstanden hat, da er zunächst das Ganze bzw. die Anzahl aller kontrollierten Fahrräder berechnet und diese als Ergebnis der Aufgabe interpretiert. Während er einen Antwortsatz auf die Frage in der Aufgabenstellung formuliert (11-13) bemerkt er seinen Fehler. Es ist anzunehmen, dass er sich bewusst ist, die Anzahl aller kontrollierten Fahrräder berechnet zu haben, und genau das das Ziel seines Lösungsweges war. Während er seinen Antwortsatz notiert und dazu Bezug zum Aufgabenstellung nimmt, erkennt er jedoch sofort, dass er nicht die Frage nach der Anzahl der mängelfreien Fahrräder, sondern die Fragen nach der Anzahl aller kontrollierten Fahrräder beantwortet hat. Erneut kann er umgehend seinen Fehler korrigieren, indem er die Differenz zwischen der Anzahl aller kontrollierten Fahrräder und der Anzahl der mangelhaften Fahrräder berechnet. Es ist zu vermuten, dass in ähnlichen Aufgabenstellungen, die im Unterricht behandelt wurden, stets nach dem Ganzen gefragt wurde, da dies ein übliches Aufgabenformat ist, bei dem ein Zusammenhang zwischen Teil und Ganzem hergestellt wurde. Vor diesem Erfahrungshintergrund ist es möglich, dass er die Aufgabenstellung nicht wortgetreu gelesen hat und entsprechend nicht erkannt hat, dass nicht nach dem Ganzen, sondern nach dem komplementären Anteil gefragt wird. Diese Vermutung wird dadurch gestützt, dass er beim Schreiben seines Antwortsatzes, wobei er sich 
an der Formulierung der Aufgabenstellung orientiert, seinen Fehler sofort erkennt und diesen korrigieren kann.

Während Julius die Lösung von Bennet abschreibt, fragt er seinen Partner was der Ausdruck ,keine Mängel“ (26-27) bedeutet und erklärt, dass er nicht wisse, was dieser bedeutet. Da er im gesamten Bearbeitungsprozess vor allem die Rolle eines Beobachters einnimmt und seine Teilnahme an der Bearbeitung sich auf Nachfragen nach Rechenschritten und Notationen beschränken, ist anzunehmen, dass er tatsächlich nicht weiß was dieser Ausdruck bedeutet. In diesem Zusammenhang kann seine Passivität im Lösungsprozess als mangelndes sprachliches Verständnis der Aufgabenstellung gedeutet werden.

Insgesamt kann die Bearbeitung von Bennet und Julius in folgenden Deutungshypothesen zusammengefasst werden:

- Durch flexibles Anpassen des Operatorschemas gelingt es Bennet von dem vorgegebenen Anteil zunächst einen Teil des Ganzen, das Ganze selber sowie einen anderen Anteil des Ganzen zu bestimmen und diesen zu berechnen. Dabei interpretiert er die angegebenen Anteile durchgehend als Größen und rechnet mit diesen auf symbolischer Ebene. Vor allem vor dem Hintergrund der wiederholten Korrekturen seines Lösungsweges ist zu erkennen, dass er sehr sicher die Zusammenhänge zwischen Teil, Anteil und Ganzem herstellen und diese auf eine rechnerische Ebene übertragen kann.

- Eine zentrale Schwierigkeit bei der Bearbeitung ist das Verständnis der Aufgabenstellung. In der Bearbeitung von Bennet und Julius konnte dies in zwei Ausprägungen beobachtet werden. Im Fall von Julius ist anzunehmen, dass er die Aufgabenstellung sprachlich nicht versteht. Er bittet seinen Partner ihm das Wort „Mängel“ zu erklären, da er dieses nicht kenne. Da dieses Wort wesentlich für das Verständnis der Aufgabenstellung ist, nimmt er lediglich passiv am Bearbeitungsprozess teil. Im Fall von Bennet ist anzunehmen, dass er im Sinne einer Übergeneralisierung früherer Aufgaben, in denen von einem Teil auf das Ganze geschlossen werden sollte, die Aufgabenstellung falsch interpretiert und annimmt, dass das Ganze bestimmt werden soll. Bei der Notation seines Antwortsatzes, für die er sich an der Fragestellung im Aufgabentext orientiert, bemerkt er seinen Fehler und korrigiert diesen umgehend.

\section{Anna Lena \& Ellen - Pfeilschema}

Anna Lena und Ellen arbeiten über die gesamte Zeit sehr konzentriert und interaktiv. In allen Bearbeitungen besprechen sie sehr detailliert ihre Lösungsansätze und -vorschläge und stellen stets sicher, dass beide Partner alles verstanden haben. Obgleich die Vortestergebnisse der beiden Schülerinnen von 40\% (Anna Lena) 
und $90 \%$ (Ellen) auf deutliche Unterschiede im Vorwissen hindeuten, sind diese in ihren Bearbeitungsprozessen nicht zu erkennen. Beide Schülerinnen tragen in gleicher Weise zur Bearbeitung der Aufgaben bei. Ihre vorhergehenden Aufgabenlösungen lassen dabei erkennen, dass sich sehr nah am Vorgehen im Lösungsbeispiel orientieren. So haben sie z. B. Anteile von Größen stets anhand eines Pfeilschemas berechnet und dargestellt. In ihren Bearbeitungsprozessen der vorhergehenden Aufgaben konnten mit Ausnahme vereinzelter Rechenfehler keine nennenswerten Schwierigkeiten der beiden Schülerinnen festgestellt werden. Das folgende Transkript dokumentiert ihre Bearbeitung von Aufgabe 2.9.

\section{Transkript B2A9 - Anna Lena \& Ellen - Szene 1 - Aufgabe 9}

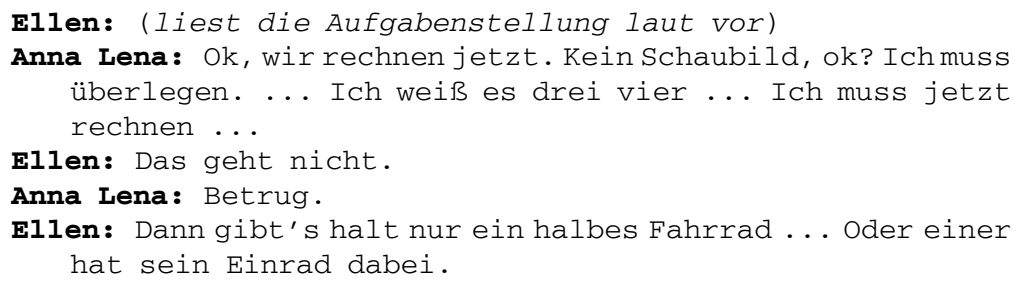

Ellen: Ich kapier das auch nicht. Wieso haben wir denn da ein halbes Fahrrad? . . J Ja, echt, das müssten 35 sein. 34 durch 7 geht ja auch nicht mal.

Nachdem Ellen die Aufgabenstellung laut vorliest, sagt Anna Lena umgehend, dass sie ,,jetzt rechnen“ müssen und schlägt vor in dieser Aufgabe ,kein Schaubild“ zu zeichnen, womit sie sich auf die Darstellung der Rechnung in einem Pfeilschema bezieht (2-4). Ellen versucht zunächst 34 durch $7 \mathrm{zu}$ teilen, stellt dann aber fest, dass diese Division nicht ganzzahlig aufgeht: „Das geht nicht“ (5).

Anna Lena blättert zunächst über die vorhergehenden Aufgaben und versichert sich der Teiloperatoren zur Anteilberechnung bzw. der Bedeutung des Zählers und Nenners eines Bruchoperators: „Immer mal das untere.. Nee, durch das untere“ (11-13). Daraus schließt sie auf den gleichen Rechenweg wie ihre Partnerin und stimmt ihr zu: „Ja, durch 7“ (13). Auch sie kommt bei dieser Rechnung nicht auf ein ganzzahliges Ergebnis und schließt mögliche Rechenfehler aus (13-14). 
Ohne dass Ellen zu Beginn sagt, was sie rechnet, sind sich die beiden Partnerinnen über den Lösungsansatz einig. Sie versuchen beide die vorgegebene Anzahl von 34 Fahrrädern entsprechend des zuvor wiederholt angewendeten Verfahrens zur Berechnung von Anteilen durch sieben zu teilen. Beide Schülerinnen stellen dabei fest, dass diese Division kein ganzzahliges Ergebnis hat. Sie stellen direkt eine Beziehung zum Aufgabenkontext her und interpretieren das Ergebnis ihrer Rechnung im Sachkontext: „Dann gibt's halt nur ein halbes Fahrrad ... Oder einer hat sein Einrad dabei“" (7-8). Während Ellen unmittelbar nach dem Lesen der Aufgabenstellung beginnt zu rechnen, muss Anna Lena noch einmal auf die vorhergehenden Aufgaben zurückgehen, um sich des genauen Vorgehens bei der Anteilberechnung zu versichern. Es kann einerseits angenommen werden, dass Anna Lena noch sehr unsicher bei der Wahl der Rechenoperationen ist, während Ellen das Verfahren an dieser Stelle bereits soweit verinnerlicht hat, dass sie sofort die Rechenoperationen zur Anwendung des Operators $\cdot \frac{2}{7}$ ableiten kann. Ungeachtet dessen, stellen beide Schülerinnen fest, dass ihr Rechenansatz nicht richtig sein kann. Ellen erklärt: „Ich kapier das auch nicht. Wieso haben wir denn da ein halbes Fahrrad? ... Ja echt, das müssten 35 sein. 34 durch 7 geht auch nicht mal“" (15-17).

\section{Transkript B2A9 - Anna Lena \& Ellen - Szene 2 - Aufgabe 9}

Nach einer kurzen Phase, in der die beiden Schülerinnen still für sich nachdenken stellt Anna Lena fest: „Ah, wir haben was völlig falsches gerechnet“ (18). Sie erklärt weiter, dass die Zahl 34 für die Anzahl der Fahrräder steht, ,die Mängel haben“ (20) und „genau anders herum“ (21) rechnen müssen. Sie erkennt, dass 34 Fahrräder zwei Teilen entsprechen (Zeile 22: „Also 2 sind ja diese 34“) und sie 
diese Anzahl demnach halbieren müssen, um $\frac{1}{7}$ des Ganzen zu erhalten: „Was ist die Hälfte davon? Was ist 34 durch 2?“" (24).

Ellen stimmt Anna Lena zu und nennt „18“ (26) als die Hälfte von 34, woraufhin Anna Lena hinzufügt, dass der weitere Rechenweg „18 mal 5“ (29) sei. Ellen übernimmt diese Rechnung und kommt zu dem Ergebnis ,90 Fahrräder ... Ja, 90 Fahrräder“. Beide Schülerinnen notieren im Stillen ihre Lösung.
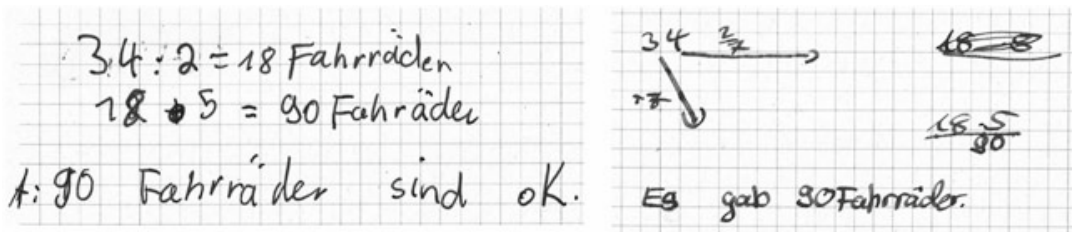

Abbildung 5.33 Anna Lenas (links) und Ellens (rechts) Lösung von Aufgabe 2.9

Ungeachtet ihres Rechenfehlers $34: 2=18$ ist in der Bearbeitung von Anna Lena und Ellen deutlich zu erkennen, dass sie zunächst versuchen das Verfahren zur Anteilberechnung direkt auf die Zahlen in der Aufgabenstellung anzuwenden und $\frac{2}{7}$ von 34 Fahrrädern berechnen wollen. Da die Division durch sieben jedoch zu keinem ganzzahligen Ergebnis führt, folgern sie, dass ihr Ansatz falsch sein muss. Nach kurzem Überlegen stellt Anna Lena dann fest, dass die angegebene Anzahl von 34 Fahrrädern nicht die Anzahl aller kontrollierten Fahrräder ist, sondern der Anteil $\frac{2}{7}$ von 34 Fahrrädern ist und berechnen müssen, an wie vielen Fahrrädern keine Mängel festgestellt wurden. Sie leitet richtig ab, dass wenn $\frac{2}{7}$ der Fahrräder mangelhaft waren, der Anteil der Fahrräder ohne Mängel dann $\frac{5}{7}$ entsprechen müssen. Um dies zu Berechnen teilt Anna Lena dann 34 durch zwei, um $\frac{1}{7}$ zu erhalten und multipliziert danach mit fünf, um $\frac{5}{7} \mathrm{zu}$ erhalten.

Obwohl Anna Lena zu Beginn noch einmal nachsehen muss, welche Rechenoperationen mit dem Zähler und Nenner durchgeführt werden, gelingt es ihr einen Zusammenhang zwischen dem vorgegebenen Anteil, einem Teil und dem komplementären Anteil herzustellen. Dabei erscheint es ihr der naheliegendste Weg zu sein $\frac{1}{7}$ des Ganzen zu berechnen, was darauf hindeutet, dass sie die Rechenoperationen und Brüche im Zusammenhang richtig interpretiert und das ursprüngliche Verfahren flexibel an die neue Situation anpasst. Dieser Eindruck wird auch durch ihre schriftliche Formulierung der Lösung bestärkt, in der sie anders als in allen vorhergehenden Aufgaben kein Pfeilschema zeichnet, sondern ihre schrittweise Rechnung in Termen notiert. 
In der Kommunikation der beiden Schülerinnen wird nicht deutlich, inwieweit Ellen den Lösungsweg von Anna Lena nachvollzieht. Anders als ihre Partnerin notiert sie nicht den Rechenweg, sondern schreibt lediglich die schriftliche Multiplikation 18 . 5 sowie einen Antwortsatz auf. Ihr Antwortsatz deutet jedoch darauf hin, dass sie den Lösungsweg möglicherweise nicht verstanden hat. Sie schreibt „Es gab 90 Fahrräder“ (siehe Abb. 5.33). Dieser Antwortsatz kann unterschiedlich interpretiert werden. Einerseits kann dieser Satz bedeuten, dass sie den Lösungsweg ihrer Partnerin nicht verstanden hat, und annimmt, dass in der Fahrradkontrolle insgesamt 90 Fahrräder geprüft wurden. Andererseits ist es möglich, dass sie mit „Es gab 90 Fahrräder" zum Ausdruck bringen will, dass es 90 Fahrräder gab, an denen keine Mängel festgestellt wurden. Auf Grundlage der vorliegenden Daten kann dies jedoch nicht entschieden werden.

Die Bearbeitung von Anna Lena und Ellen kann in den folgenden Deutungshypothesen zusammengefasst werden:

- Anna Lena und Ellen versuchen zunächst das Operatorschema anzuwenden und $\frac{2}{7}$ von 34 zu berechnen, ohne einen direkten Bezug zur Situation im Aufgabenkontext herzustellen. Da diese Rechnung zu keinem plausiblen Ergebnis führt, suchen sie nach einem neuen Lösungsansatz.

- Obwohl Anna Lena zu Beginn Unsicherheiten bezüglich der Anwendung des Operatorschemas gezeigt hat, gelingt es ihr in der Folge das Verfahren flexibel an die Sachsituation anzupassen und über die Bestimmung eines Teils des Ganzen den gesuchten Anteil zu berechnen.

- Das Pfeilschema wird zur direkten Anwendung des Verfahrens genutzt. Anna Lena und Ellen setzen zunächst an $\frac{2}{7}$ von $34 \mathrm{zu}$ berechnen und beginnen die Rechnung in einem Pfeilschema darzustellen. Als diese Rechnung jedoch zu keinem Ergebnis führt und sie den Rechenweg anpassen, wählen sie eine Termschreibweise zur Notation ihrer mehrschrittigen Rechnungen.

\section{Julia \& Marie - Fehlvorstellung von Anteilen}

Die Partnerarbeit von Julia und Marie wird stark von Marie geleitet. Die Vortestergebnisse der beiden Schülerinnen zeigten beträchtliche Unterschiede in ihren Vorkenntnissen. Während Julia lediglich $20 \%$ der Aufgaben im Vortest korrekt lösen konnte, erreichte Marie $75 \%$ der möglichen Punkte. In den vorhergehenden Aufgabenlösungen zeigte Julia wiederholt, dass sie noch große Unsicherheiten bei der Deutung von Brüchen hat und sehr unsicher beim Berechnen von Anteilen ist. Die unterschiedlichen Leistungsniveaus werden insbesondere darin deutlich, dass Marie die Aufgaben größtenteils eigenständig löst. Julia versucht dabei ihrer Partnerin zu folgen und stellt viele Nachfragen, die von Marie ausführlich beantwortet werden. 


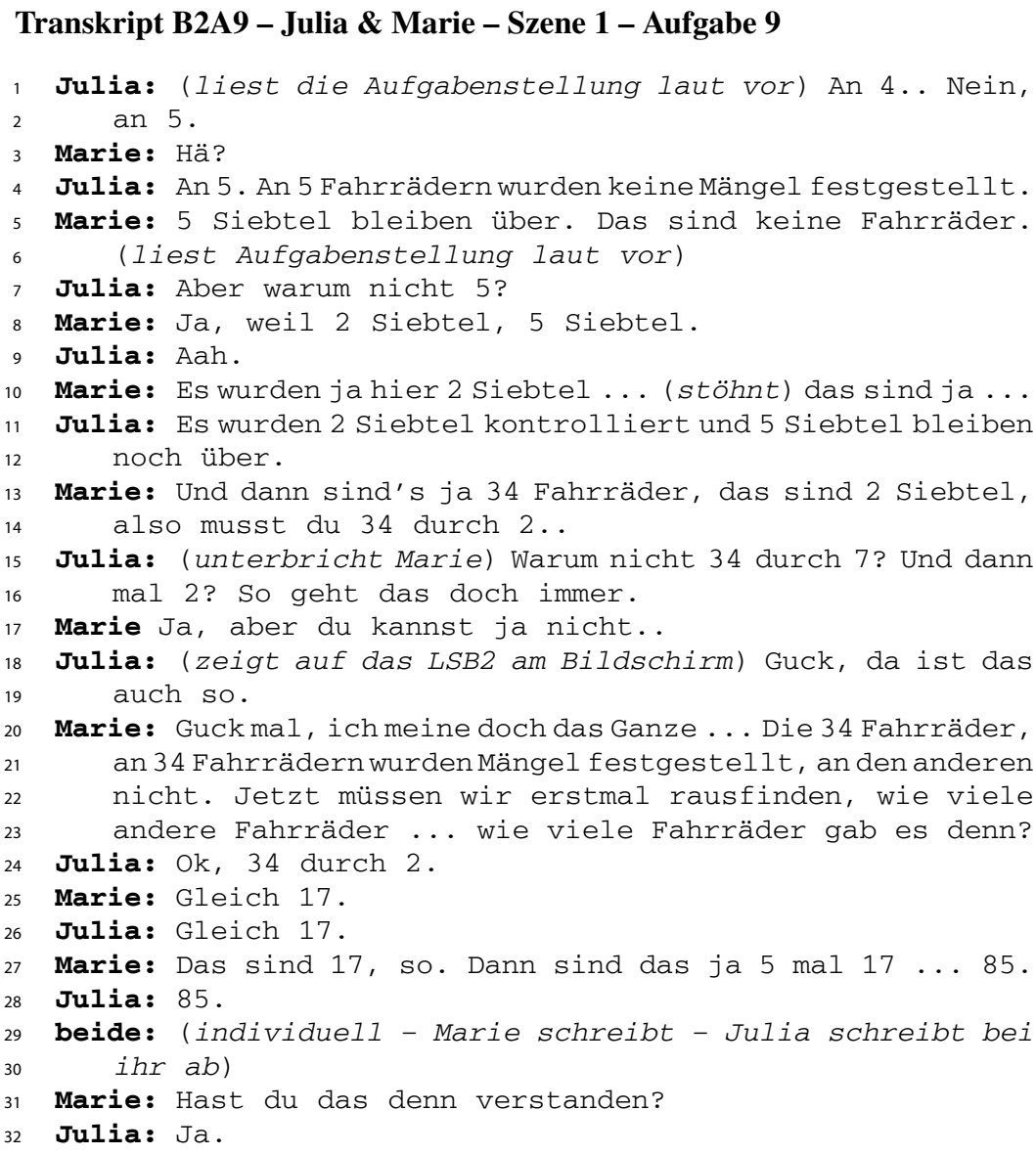

Julia liest zunächst die Aufgabenstellung laut vor und sagt sofort, dass die Antwort auf die Frage, an wie vielen Fahrrädern keine Mängel festgestellt wurden, ,5“ sei: „An 5 Fahrrädern wurden keine Mängel festgestellt“ $(1-2,4)$. Ihre Partnerin Marie widerspricht diesem Vorschlag und erklärt, dass ,5 Siebtel“ übrig bleiben und die Siebtel „keine Fahrräder“ seien (5). Julia scheint diese Erwiderung nicht zu verstehen und fragt „warum nicht 5?“ (7), worauf Marie ihr erklärt, dass es „2 Siebtel, 5 Siebtel“ (8) sind. Julia nimmt diese Argumentation auf und interpretiert sie so, dass „2 Siebtel kontrolliert [wurden] und 5 Siebtel noch über“ (12-13) bleiben. 
Julias Lösungsvorschlag, dass an nur fünf Fahrrädern keine Mängel festgestellt wurden, deutet darauf hin, dass sie die Brüche nicht als Anteile eines Ganzen, sondern die Zähler der Brüche als natürliche Zahlen interpretiert. Ihrer Argumentation zufolge interpretiert sie die Aufgabensituation so, dass 7 Fahrräder kontrolliert wurden, und an 2 dieser Fahrrädern Mängel festgestellt wurden. Entsprechend müssen fünf dieser Fahrräder ohne Mängel gewesen sein. Diese Interpretation ist auf anschaulicher Ebene mit der verbreiteten Fehlvorstellung $\frac{1}{n}$ bezeichnet eine Menge von $n$ Objekten als Übergeneralisierung des Quasikardinalzahlaspekts von Brüchen vergleichbar. Demnach übersetzt sie den Bruch $\frac{2}{7}$ als 2 und den Bruch $\frac{5}{7}$ als 5 . Es ist anzunehmen, dass sie nur bedingt eine anschauliche Vorstellung zu diesen Brüchen aktivieren kann und in der Folge und in Bezug auf die Fragestellung in der Aufgabenstellung, in der nach einer Anzahl von Fahrrädern gefragt wird, auf die natürlichen Zahlen ausweicht. Auch ihre Antwort auf die Erwiderung ihrer Partnerin, dass ,2 Siebtel kontrolliert [wurden] und 5 Siebtel [...] noch über [bleiben]“ (12-13) unterstützt diese Annahme. Bei wörtlicher Interpretation dieser Aussagen müsste angenommen werden, dass Julia meint, dass insgesamt $\frac{2}{7}$ Fahrräder kontrolliert wurden und demnach noch $\frac{5}{7}$ Fahrräder nicht kontrolliert wurden, was darauf hindeutet, dass Julia keinen Bezug zum Ganzen herstellt bzw. $\frac{2}{7}$ als Ganzes interpretiert. Diese Äußerung kann jedoch auch so interpretiert werden, dass sie meint, dass $\frac{2}{7}$ und $\frac{5}{7}$ zusammen das Ganze ergeben.

Auf Maries Feststellung, dass 34 Fahrräder $\frac{2}{7}$ sind und sie folglich $34: 2$ rechnen müssen (14-15) unterbricht Julia ihre Partnerin und fragt, warum sie nicht ,34 durch 7 [...] und dann mal 2“ (16-17) rechnen, da „das doch immer" so gerechnet würde. Hier wird deutlich, dass Julia nun den Anteil $\frac{2}{7}$ von 34 berechnen möchte und für diesen Fall auch die korrekten Rechenoperatoren bestimmt, also in dieser Situation das Verfahren der Anteilberechnung übertragen möchte. Da sie jedoch die Aufgabensituation und insbesondere die Beziehungen zwischen Anteil, Teil und Ganzem nicht verstanden hat, versteht sie nicht, warum Marie etwas anderes rechnen möchte. Julia verweist weiterhin auf das Lösungsbeispiel am Bildschirm und stützt ihr Argument damit, dass im Lösungsbeispiel auch so gerechnet werde (19-20).

Im Gegensatz zu Julia scheint Marie die Aufgabensituation und insbesondere den Zusammenhang zwischen Anteil, Teil und Ganzem sofort zu erkennen. Sie halbiert in der Folge $\frac{2}{7}$ bzw. 34 Fahrräder um zu berechnen, dass $\frac{1}{7}$ des Ganzen 17 Fahrrädern entspricht. Diesen Teil multipliziert sie danach mit fünf um $\frac{5}{7}$ des Ganzen bzw. 85 Fahrräder als Anzahl der mangelfreien Fahrräder zu erhalten (21-29).

Insgesamt kann die Bearbeitung von Julia und Marie wie folgt zusammengefasst werden: 
- Julia interpretiert die Brüche $\frac{2}{7}$ und $\frac{5}{7}$ nicht als relative Anteile einer Menge, sondern entsprechend der Fehlvorstellung $\frac{m}{n}$ bedeutet $m$ (siehe Abschnitt 2.2.4) als absolute Anteile (bzw. Stückzahlen) 2 und 5 Fahrräder (Übergeneralisierung des Quasikardinalzahlaspekts von Brüchen).

- Julia möchte den Anteil $\frac{2}{7}$ von 34 berechnen und nennt auch die dazu notwendigen Rechenoperatoren. Damit zeigt sie, dass die das Verfahren zur Anteilberechnung überträgt und anwendet. Ihre Äußerungen deuten darauf hin, dass sie diese Rechenschritte soweit verallgemeinert hat, dass sie sagt, dass immer auf diese Weise gerechnet würde, und nicht versteht, warum ihre Partnerin in dieser Situation anders rechnen möchte.

- Aufgrund ihrer Fehlvorstellung zu den angegebenen Brüchen gelingt es Julia nicht den Anteil, einen Teil und das Ganze zu identifizieren und damit die Aufgabensituation zu verstehen.

- Marie hat keine Schwierigkeiten, die Aufgabensituation zu erschließen und das Verfahren zur Anteilberechnung anzupassen. Sie berechnet korrekt aus dem Anteil des Ganzen einen Teil des Ganzen, um von diesem ausgehend den gesuchten Anteil zu berechnen.

\section{Enya \& Luisa - Fehlerhafter Bezug zum Ganzen}

Enya und Luisa gehören zu den leistungsstarken Schülerinnen und Schülern der Lerngruppe. Bereits im Vortest konnten sie $85 \%$ (Enya) und $90 \%$ (Luisa) der Aufgaben korrekt lösen. In den vorhergehenden Aufgaben zeigten sie keine besonderen Schwierigkeiten und konnten alle Aufgaben korrekt lösen.

\section{Transkript B2A9 - Enya \& Luisa - Szene 1 - Aufgabe 9}

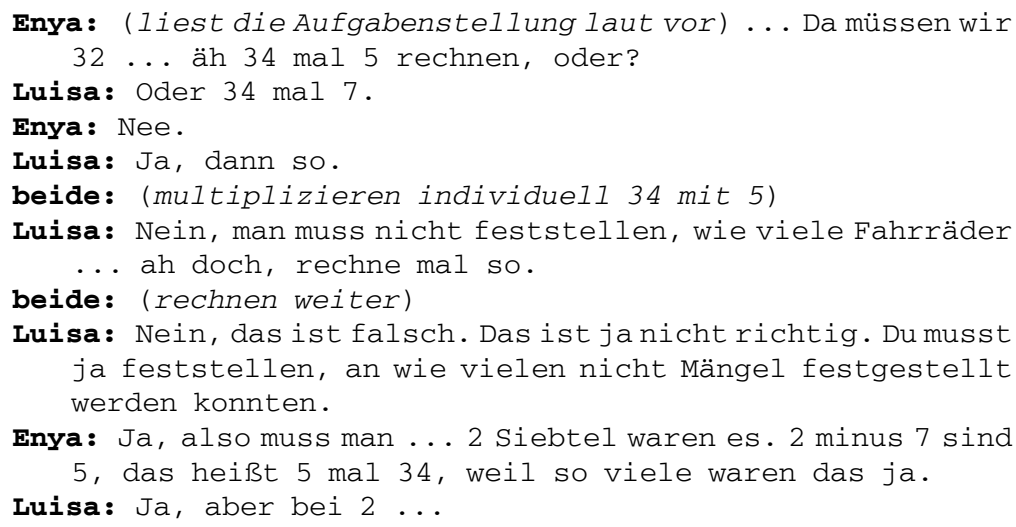


16

Die Bearbeitung von Enya und Luisa beginnt damit, dass Enya die Aufgabenstellung laut vorliest und nach kurzem Überlegen vorschlägt: ,da müssen wir [...] 34 mal 5 rechnen“ (1-2). Sie interpretiert 34 Fahrräder bzw. $\frac{2}{7}$ der Fahrräder als Einheit und hat erkannt, dass zwei Teile durch fünf Teile zu einem Ganzen ergänzt werden. Somit folgert sie, dass fünf Einheiten die gesuchte Menge an Fahrrädern ohne Mängel ergeben.

Ihre Partnerin Luisa schlägt alternativ vor zunächst die Anzahl aller kontrollierten Fahrräder bzw. das Ganze zu bestimmen und 34 mit 7 zu multiplizieren (3). Ihr Alternativvorschlag deutet darauf hin, dass sie genau wie ihre Partnerin 34 Fahrräder als Einheit betrachtet und nicht erkennt, dass $\frac{2}{7}$ bereits zwei Teile beinhaltet.

Luisas Vorschlag wird von Enya abgelehnt und so multiplizieren die beiden Schülerinnen im Stillen 34 mit 7. Während der Ausführung dieser Rechnung hält Luisa inne und äußert Zweifel an ihrem Vorgehen: „Nein, muss man nicht feststellen, wie viele Fahrräder ... ah doch rechne mal so“ (7-8). Unmittelbar nach der Fertigstellung ihrer Rechnung führt sie ihre Zweifel genauer aus und erklärt: „Nein, das ist falsch. Das ist ja nicht richtig. Du musst ja feststellen, an wie vielen nicht Mängel festgestellt werden konnten“ (10-12). Demnach deutet sie ihre Rechnung nicht als Berechnung des komplementären Anteils, sondern nimmt an, dass sie durch eine Multiplikation mit 5 die Anzahl aller kontrollierten Fahrräder bestimmt.
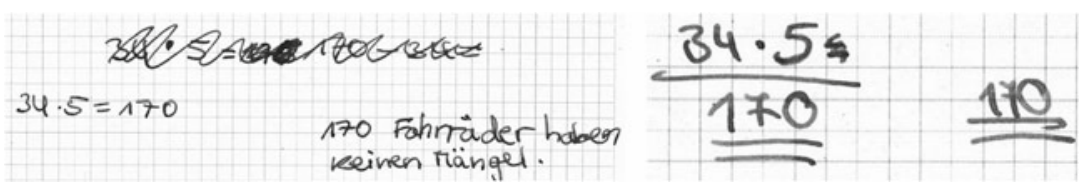

Abbildung 5.34 Luisas (links) und Enyas (rechts) Lösung von Aufgabe 2.9

Die schriftliche Dokumentation ihrer Bearbeitung stützt diese Deutung (vgl. 5.34). Nachdem sie $34 \cdot 7=170$ berechnet hat, setzt sie an 34 von 170 zu subtrahieren. Sie führt diese Rechnung jedoch nicht aus und streicht den Term im weiteren wieder durch. Dieser Ansatz bestärkt einerseits die Vermutung, dass sie annimmt durch eine Multiplikation von 34 mit 5, die Anzahl aller kontrollierten Fahrräder zu erhalten. Zudem ist hier zu erkennen, dass sie unter der Annahme, dass insgesamt $\frac{7}{7} 170$ Fahrrädern entsprechen, sie $\frac{2}{7}$, also 34 Fahrräder abziehen möchte, um $\frac{5}{7}$ zu erhalten. Dies ist insbesondere bemerkenswert, da sie offenbar 34 Fahrräder als $\frac{2}{7}$ des Ganzen erkennt, jedoch keine Rückschlüsse auf ihren Lösungsansatz zieht. 
Enya widerspricht dem Einwand von Luisa und begründet: „,2 Siebtel waren es. 2 minus 7 sind 5, das heißt 5 mal 34, weil so viele waren es ja" (13-14). Auch in dieser Begründung von Enya ist zu erkennen, dass sie korrekt den komplementären Anteil zu $\frac{2}{7}$ bestimmen, dann jedoch $\frac{2}{7}$ bzw. 34 Fahrräder als Einheit bzw. als $\frac{1}{7}$ betrachten und mit diesem Wert weiter rechnet.

Luisas erneuten Einwand „Ja, aber bei 2“ (15) ignoriert sie und setzt sich letztlich durch, indem sie ihre Begründung fortführt und erklärt, dass es dann ,,170 Fahrräder [sind], die keinen Mangel hatten“ (16-17).

Die Bearbeitung von Enya und Luisa kann in folgender Deutungshypothese zusammengefasst werden:

- Das Verfahren zur Anteilberechnung wird direkt auf die gegebenen Zahlenwerte übertragen und angewendet. Enya und Luisa interpretieren den vorgegebenen Zahlenwert von 34 Fahrrädern als Einheit bzw. als $\frac{1}{7}$ der kontrollierten Fahrräder und nehmen diesen als Grundlage für ihre Rechnung. Sie erkennen nicht, dass diese 34 mangelhaften Fahrräder selber bereits ein Anteil sind und multiplizieren diese Anzahl gemäß des bekannten Verfahrens zur Anteilberechnung mit 5.

\section{Philip \& Can - Übergeneralisierung des Verfahrens zur Anteilberechnung und Plausibilisierung einer falschen Rechnung sowie des Ergebnisses}

Im Anschluss an die geschilderte Bearbeitung der unvollständigen Beispiele war die Partnerarbeit von Can und Philip weiterhin von vielen Ablenkungen geprägt. In den auf die unvollständigen Beispiele folgenden Aufgaben waren zwei Aufgaben enthalten, in denen Anteile von Größen berechnet werden sollten. In diesen sollten $\frac{4}{9}$ und $\frac{5}{9}$ von 90 Kilometern, $\frac{1}{4}$ von 256 Kilogramm und $\frac{3}{4}$ von 6 Metern berechnet werden. Can und Philip haben keine dieser Aufgaben erfolgreich lösen können und in allen Fällen nach einem ersten Lösungsversuch aufgegeben. Im Gegensatz dazu haben sie in einer Aufgabe zum Kontrollieren von vorgegebenen Berechnungen von Anteilen den enthaltenen Fehler korrekt identifiziert, konnten diesen jedoch nicht korrigieren. Die Analyse ihrer vorhergehenden Bearbeitungsprozesse zeigt, dass sie das Verfahren zur Berechnung von Anteilen beliebiger Größen nicht selbstständig auf neue Aufgabensituationen und Sachkontexte übertragen haben. Das nachfolgende Transkript dokumentiert ihre Bearbeitung von Aufgabe 2.9.

\section{Transkript B2A9 - Can \& Philip - Szene 1 - Aufgabe 9}

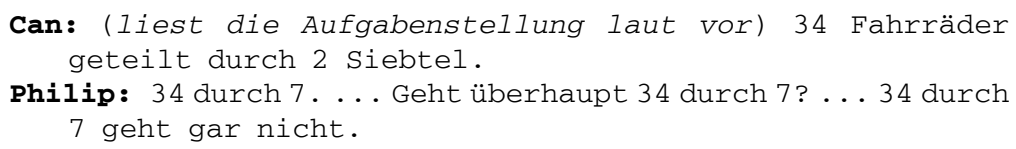




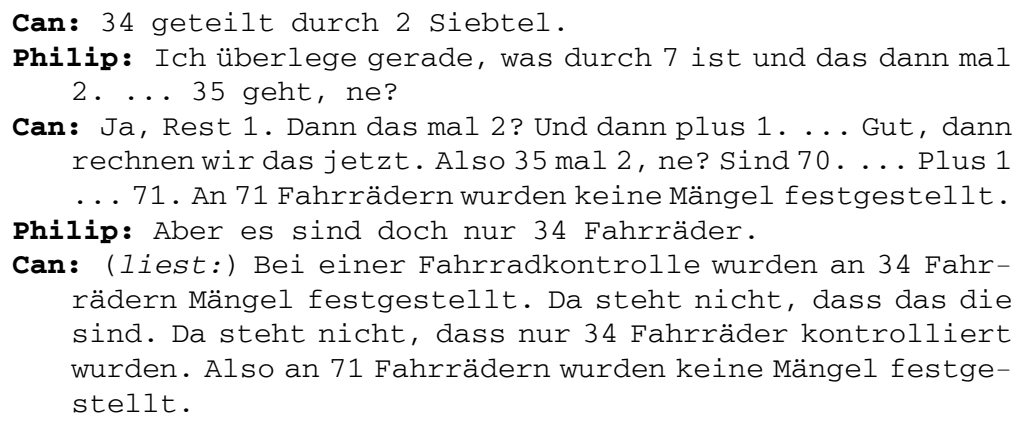
rädern Mängel festgestellt. Da steht nicht, dass das die sind. Da steht nicht, dass nur 34 Fahrräder kontrolliert wurden. Also an 71 Fahrrädern wurden keine Mängel festgestellt.

beide: (schreiben individuell)

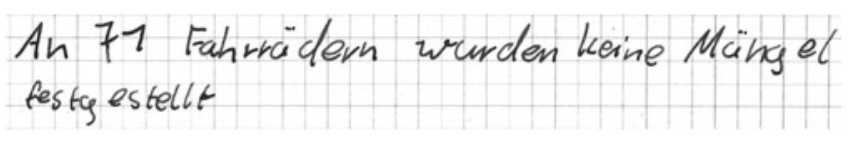

Abbildung 5.35 Philips Lösung von Aufgabe 2.9

Direkt nachdem Can die Aufgabenstellung laut vorgelesen hat, versucht er das Verfahren zur Berechnung von Anteilen anzuwenden und schlägt vor „34 Fahrräder geteilt durch 2 Siebtel" (1-2) zu rechnen. Philip beginnt sofort zu rechnen und teilt „,34 durch 7“ (3). Er stellt jedoch fest, dass 34 nicht durch sieben teilbar ist, woraufhin Can wiederholt, dass sie „,34 geteilt durch 2 Siebtel“ rechnen müssen.

Beide Schüler versuchen somit das Verfahren Anteilberechnung anzuwenden und $\frac{2}{7}$ von $34 \mathrm{zu}$ berechnen. Während Philip jedoch direkt beginnt zu rechnen und versucht 34 durch sieben zu teilen, geht Can nicht auf die Feststellung seines Partners ein, dass 34 nicht durch 7 teilbar ist. Can selber versucht nicht den Anteil auszurechnen, was vor dem Hintergrund der vorhergehenden Bearbeitungen, insbesondere der unvollständigen Beispiele, die darauf hindeuten könnten, dass er Schwierigkeiten bei der Durchführung einer solchen Anteilberechnung hat.

Es ist zudem zu erkennen, dass Can und Philip zunächst keinen Bezug zur Aufgabenstellung herstellen und dem Aufgabentext lediglich die Zahlenwerte 34 und $\frac{2}{7}$ entnehmen, auf die sie das Operatorschema anwenden wollen. Sie hinterfragen dabei nicht, was diese Zahlen im Sachkontext bedeuten.

Philip versucht weiter den Anteil $\frac{2}{7}$ von 34 auszurechnen und nennt auch korrekt die dazu notwendigen Teiloperatoren geteilt ,,durch 7“ und ,mal 2“. Erneut bemerkt er, dass 34 nicht durch sieben teilbar ist, jedoch 35 durch 7 teilbar sei. Diesen Gedanken nimmt Can auf und interpretiert ihn so, dass 34 bei der Division durch 
sieben den Rest eins lässt und somit die Rechnung aufgehe (8). Er formuliert darauf aufbauend einen neuen Rechenweg: ,Dann das mal 2? Und dann plus 1 [...] Also 35 mal 2 [...] sind 70 [...] plus 1 .. 71“ (8-11). Can erkennt nicht, dass 34 bei der Division durch sieben nicht den Rest eins, sondern sechs lässt. Unabhängig davon geht er nun von 35 als Bezugsgröße aus und multipliziert diese entsprechend des zweiten Teiloperators mit 2. Er kommt zu dem Ergebnis 70 und addiert auf diesen noch den vermeintlichen Rest eins.

Obwohl seine Rechnung den korrekten zweiten Teiloperator zum Berechnen des Anteils von $\frac{2}{7}$ von einer Größe enthält, kann Cans Rechnung so interpretiert werden, dass er vermeintlich willkürlich versucht eine Rechnung aufzustellen, die diesen Teiloperator enthält. Den ersten Teiloperator :7 erwähnt er zu keinem Zeitpunkt, was die Annahme seiner Schwierigkeiten bei der Anwendung des Verfahrens weiter bestärkt. Er plausibilisiert seinen Rechenweg im weiteren dadurch, dass er den vermeintlichen Rest eins auf sein Ergebnis addiert, obgleich er von seinem Ergebnis eigentlich das Doppelte dieses Rests subtrahieren müsste.

Im Anschluss setzt er seine Lösung in Bezug zum Aufgabenkontext und erklärt auf den Einwand von Philip, dass es ,doch nur 34 Fahrräder“ (12) seien, dass in der Aufgabenstellung nicht stehe, ,,dass nur 34 Fahrräder kontrolliert wurden“, sondern ,,an 34 Fahrrädern Mängel festgestellt wurden“ (13-16). Im Aufgabenkontext erscheint ihm sein Ergebnis plausibel und er formuliert den Antwortsatz „An 71 Fahrrädern wurden keine Mängel festgestellt" (16-17), den Philip übernimmt und im Arbeitsheft notiert (vgl. Abb. 5.35).

Philips Einwand, dass „es doch nur 34 Fahrräder“ (12) seien, bringt zum Ausdruck, dass auch er, wie zu Beginn vermutet, die Aufgabensituation nicht richtig versteht, sondern annimmt, dass die Aufgabe darin besteht, $\frac{2}{7}$ von $34 \mathrm{zu}$ berechnen. Er ist zunächst irritiert, dass Cans Ergebnis größer ist als die von ihm angenommene Gesamtmenge der kontrollierten Fahrräder, lässt sich dann aber von Can überzeugen und übernimmt dessen Ergebnis.

Insgesamt lässt sich die Bearbeitung von Can und Philip in den folgenden Deutungshypothesen zusammenfassen:

- Die Aufgabensituation wird von Can und Philip nicht vollständig erschlossen. Sie entnehmen dem Aufgabentext lediglich die Zahlenwerte $\frac{2}{7}$ und 34, die sie so interpretieren, dass die Aufgabe darin besteht, den Anteil $\frac{2}{7}$ von 34 zu berechnen. Dieses Vorgehen kann als Übergeneralisierung des Verfahrens zur Anteilberechnung verstanden werden. Can und Philip übertragen das Vorgehen aus den vorhergehenden Aufgaben, ohne dass sie die Aufgabenstellung in ihre Überlegungen mit einbeziehen. 
- Philip nennt die für Berechnung des Anteils $\frac{2}{7}$ von 34 korrekten Rechenoperatoren und zeigt, dass er das Verfahren zur Anteilberechnung grundsätzlich anwenden kann und die erforderlichen Rechenoperationen ableiten kann.

- Da ihr Rechenweg bereits bei der Division durch 7 nicht aufgeht, stellt Can einen willkürlich wirkenden Rechenweg zusammen, anhand dem seine Schwierigkeiten bei der Anteilberechnung deutlich werden. Anstatt den Ausgangswert zunächst durch 7 zu teilen und das Ergebnis dann mit 2 zu multiplizieren, multipliziert er direkt 34 mit 2 und addiert im Anschluss eins dazu. Es wundert ihn dabei nicht, dass sein berechneter Anteil vom Ganzen größer als das Ganze ist.

- Can stellt sein Ergebnis in Beziehung zum Aufgabentext und versucht anhand diesem sein Ergebnis zu plausibilisieren. Seine Argumentation überzeugt auch Philip, der seine Lösung übernimmt.

\section{Vergleich der Bearbeitungen}

In den Analysen der Bearbeitungsprozesse der unterschiedlichen Paare ist zu erkennen, dass in allen Fällen das Verfahren zur Anteilberechnung übertragen und angewendet wird. Der Bruchoperator „, $\frac{2}{7}$ von“ wurde in nahezu allen Fällen mit dem Handlungsschema des Teilens des Ganzen in sieben gleiche Teile und dem Multiplizieren eines Teils mit zwei übersetzt. Dies ist insbesondere in den Wahlen der Rechenoperationen der Schülerinnen und Schüler zu erkennen.

Es konnte zudem beobachtet werden, dass die korrekte Interpretation des Operators kein hinreichendes Kriterium zur Anpassung des Verfahrens an die Situationseigenschaften des Aufgabenkontexts darstellt. Hierzu ist es erforderlich zu erkennen, dass die vorgegebene Anzahl von 34 Fahrrädern, an denen Mängel festgestellt wurden, ein Anteil der Menge aller kontrollierten Fahrräder ist. Nur durch die Interpretation dieser Anzahl als Anteil ist möglich, die Situation vollständig zu erschließen auf die Menge aller kontrollierten Fahrräder sowie dem Anteil der Fahrräder ohne Mängel zu schließen. Vor diesem Hintergrund konnten in den dargestellten Bearbeitungsprozessen charakteristische Fehler dokumentiert werden:

- Die direkte Anwendung des Verfahrens auf die im Aufgabentext angegebenen Zahlenwerte ohne Reflexion der Aufgabenstellung: $\frac{2}{7}$ von 34 .

- Die Interpretation von 34 Fahrrädern als $\frac{1}{7}$ des Ganzen und Multiplikation mit 5 zur Berechnung von $\frac{5}{7}$.

Allgemein kann das zentrale Fehlermuster in der Anwendung der Teiloperatoren :7 und $\cdot 2$ auf die falsche Bezugsgröße zusammengefasst werden. Diese Fehler sind somit vor allem auf eine mangelnde Interpretation der Aufgabensituation auf Grundlage der Anteilvorstellung zurückzuführen, sodass die Schülerinnen und Schüler 
falsche Auswahlen für den Anteil, einen Teil oder das Ganze getroffen haben und fehlerhafte Bezüge zwischen diesen hergestellt haben.

Die direkte Anwendung des Operators , $\frac{2}{7}$ von“ auf die Zahl 34 kann als Übergeneralisierung des Verfahrens zur Anteilberechnung interpretiert werden. Die Fokussierung auf die Anwendung dieses Handlungsschemas beschränkt in vielen Fällen den Blick auf die Aufgabensituation, sodass diese nicht vollständig erfasst und fehlerhafte Zuordnungen vorgenommen werden. Eine Anpassung des Verfahrens wurde in vielen Fällen erst dann in Betracht gezogen, als die Schülerinnen und Schüler festgestellt haben, dass die vorgegebene Anzahl von 34 Fahrrädern nicht durch sieben teilbar ist. Erst dadurch wurde in vielen Fällen erkannt, dass die 34 Fahrräder zwei von insgesamt sieben Teilen der Fahrräder darstellen. Sofern diese Verknüpfung erfolgreich hergestellt wurde, konnte beobachtet werden, dass die Schülerinnen und Schüler in der Folge einen korrekten Lösungsweg finden und die notwendigen Rechenoperationen ableiten konnten.

Die Bestimmung des gesuchten Anteils erfolgte dann in den meisten Fällen nicht über die Bestimmung des Ganzen bzw. der Menge aller kontrollierten Fahrräder, sondern über den Teil $\frac{1}{7}$ bzw. 17 Fahrräder. Nur im Fall von Bennet und Julius wurde das Ganze bestimmt, wobei Bennet zunächst angenommen hat, dass das Ziel der Aufgabe die Bestimmung des Ganzen ist. In nahezu allen anderen Fällen wurde ein Teil mit fünf multipliziert, um $\frac{5}{7} \mathrm{zu}$ erhalten, was als Kennzeichen für eine flexible Anwendung und Anpassung des Verfahrens interpretiert werden kann.

In den Bearbeitungsprozessen dieser komplexen Transferaufgabe konnten auch fehlerhafte Denkmuster bzw. grundlegende Fehlvorstellungen dokumentiert werden. Im Fall von Can und Philip führte die Beobachtung, dass 34 nicht durch sieben teilbar ist, nicht dazu, dass die Schüler ihren Lösungsansatz noch einmal reflektiert und auf die Aufgabenstellung bezogen haben. Stattdessen versuchten sie die Teiloperatoren :7 und $\cdot 2$ auf willkürlich anmutende Weise anzuwenden, um ein plausibles Ergebnis zu erhalten. Dieses Vorgehen kann dem verbreiteten Befund der ,komponentenweisen Betrachtung von Brüchen als Kombination von zwei natürlichen Zahlen“ zugeordnet werden, da die Schüler den Zähler und Nenner von $\frac{2}{7}$ nicht ganzheitlich, sondern als voneinander getrennte Objekte betrachten, die mit einer Rechenoperation verknüpft und unabhängig voneinander angewendet werden.

Im Fall von Julia und Marie konnte beobachtet werden, dass Julia vom Anteil $\frac{2}{7}$ auf den komplementären Anteil $\frac{5}{7}$ aller kontrollierten Fahrräder geschlossen hat. Sie interpretierte den Bruch $\frac{5}{7}$ in der Folge jedoch nicht als Bruchoperator, der auf eine Größe angewendet wird, sondern als absoluten Anteil. Entsprechend der verbreiteten Fehlvorstellung schloss sie aus dem Bruch $\frac{5}{7}$ darauf, dass fünf Fahrräder ohne Mängel waren. Sie bezieht den Bruch nicht als relativen Anteil auf eine Größe, sondern interpretiert den Zähler 5 im Sinne einer Übergeneralisierung des Quasikar- 
dinalzahlaspekts von Brüchen als absoluten Anteil bzw. als Anzahl von Fahrrädern. Dieser Fehler deutet auf einen Rückgriff auf die Abzählbarkeit der natürlichen Zahlen aufgrund der Ermangelung adäquater anschaulicher Vorstellungen von Brüchen als relative Anteile hin.

\subsubsection{Vergleich der Bearbeitungsprozesse in den beiden Transferaufgaben}

Die Analysen der Bearbeitungsprozesse zum Transfer des Verfahrens zum Berechnen von Anteilen beliebiger Größen in diesem Abschnitt können unter drei wesentlichen Aspekten von Transferprozessen zusammengeführt und diskutiert werden: Der Ausbildung eines Handlungsschemas, der Übertragung dieses Handlungsschemas auf eine neue Sachsituation und der Verallgemeinerung dieses Handlungsschemas.

Ausbildung eines Handlungsschemas: Bereits in den Bearbeitungsprozessen der unvollständigen Beispiele ist zu erkennen, dass die Lernenden ein Handlungsschema zu dem Ausdruck $\frac{m}{n}$ von einer Größe ausgebildet haben, das ein Teilen des Ganzen in $n$ gleiche Teile und ein $m$-faches Vervielfachen eines Teils vorsieht. In den Bearbeitungsprozessen ist deutlich zu erkennen, dass die Lernenden stets versuchen dieses Handlungsschema auf die vorgegebenen Größen anzuwenden. Die Ausbildung dieses Handlungsschemas kann als fortschreitende Schematisierung und Erweiterung der Bruchherstellungshandlung zur Anteilbildung betrachtet werden. Bereits seit der ersten Sitzung der Unterrichtseinheit wurde diese Handlungen an Repräsentanten von konkreten Gegenständen (Kreise, Rechtecke, Strecken) eingeführt und durch die Übertragung auf Maßzahlen und unterschiedliche Größenbereite erweitert.

Die Übertragung und Anwendung dieses Handlungsschemas ist in allen in diesem Abschnitt dargestellten Bearbeitungsprozessen dokumentiert. Dabei scheint die Beschaffenheit des Ganzen in keinem Fall von Bedeutung zu sein und es macht für die Lernenden scheinbar keinen Unterschied, ob das Ganze eine ikonische Figur, eine Maßzahl oder abstrakte Größe ist.

Während die Anwendung dieses Handlungsschemas auf vorgegebene Zahlen und Größen, wie z. B. $\frac{4}{7}$ von $21000 €$, allen Lernenden in den dargestellten Bearbeitungsprozessen gelingt, so stellt die Auswahl der Zahlen und Größen in einem Sachkontext die zentrale Schwierigkeit bei der Übertragung und Anwendung des Handlungsschemas auf neue Anforderungssituationen dar.

Übertragung des Handlungsschemas auf eine neue Sachsituation: In den Analysen der Bearbeitungsprozesse hinsichtlich der Übertragung und Anwendung des 
Verfahrens zur Anteilberechnung ist zu erkennen, dass die Lernenden keine Probleme bei der Übertragung und Anwendung des Verfahrens als solches auf neue Zahlen und Größen hatten. Schwieriger stellte sich diese Übertragung und Anwendung in Situationen dar, in denen die Lernenden zunächst eigenständig die entsprechenden Anteile und Bezugsgrößen einer Situationsbeschreibung entnehmen mussten. So konnten bereits in den Bearbeitungen des ersten unvollständigen Beispiels, das sich vom Lösungsbeispiel nur in den Zahlenwerten unterscheidet, Schwierigkeiten bei der Übertragung des Lösungsweges beobachtet werden. Die Analysen der Bearbeitungsprozesse der Lernenden wurden dahingehend interpretiert, dass insbesondere die äußere Gestaltung der Aufgabenstellung die Analogiebildung zum Lösungsbeispiel beeinträchtigt.

Die Schwierigkeiten der Lernenden betreffen nicht das Berechnen von Anteilen, sondern in allen Fällen die Identifizierung der Anteile als Grundlage für die Berechnung. Ungeachtet der isomorphen Analogie zwischen dem Lösungsbeispiel und dem ersten unvollständigen Beispiel stellte es sich als Schwierigkeit heraus, die veränderte Anzahl an Familienmitgliedern als Grundlage für die Aufteilung des Geldbetrags zu identifizieren. Es konnte wiederholt beobachtet werden, dass die Lernenden die Möglichkeit in Betracht zogen, den Geldbetrag zu gleichen Teilen auf die Familien aufzuteilen.

Im zweiten unvollständigen Beispiel sollte ein Geldbetrag auf zwei Jugendliche aufgeteilt werden, die unterschiedlich viele Stunden gearbeitet haben. Anders als zuvor sollte ein Geldbetrag nicht entsprechend der Anzahl von Personen, sondern entsprechend der Anzahl von Arbeitsstunden zweier Personen aufgeteilt werden. Hier zeigte sich erneut, dass die Lernenden keine Schwierigkeiten bei der Berechnung der Anteile hatten, sondern ihre Schwierigkeiten ausschließlich in der Bestimmung der Anteile zur Aufteilung des Ganzen zu verorten waren.

Die Analysen der Bearbeitungen der unvollständigen Beispiele deuten darauf hin, dass die Schwierigkeiten der Lernenden bei der Anteilbildung vor allem auf zwei situationsbezogene Aspekte zurückgeführt werden können: Einerseits die abstrakte äußere Gestaltung des ersten unvollständigen Beispiels, in dem im Unterschied zum Lösungsbeispiel keine Personen abgebildet sind, auf die der Geldbetrag in gleichen Teilen aufgeteilt werden soll. Zum anderen ist anzunehmen, dass die Vertrautheit mit der Sachsituation, insbesondere im Fall des zweiten unvollständigen Beispiels zu Schwierigkeiten geführt hat. Hier ist anzunehmen, dass das Aufteilen eines Arbeitslohns ein den Lernenden eher unvertrauter Erfahrungsbereich ist und sie somit nicht unmittelbar die Anzahl der Arbeitsstunden als Aufteilungsgrundlage identifiziert haben.

Im Gegensatz zu den unvollständigen Beispielen war in den Bearbeitungsprozessen der mehrschrittigen Transferaufgabe „Fahrradkontrolle“ eine Anpassung des 
Verfahrens bzw. eine Interpretation der Situation auf Grundlage des Verfahrens zur Anteilberechnung erforderlich. In den Analysen konnte rekonstruiert werden, dass die Lernenden auch hier keine Schwierigkeiten bei der Berechnung von Anteilen hatten, sondern ihre Schwierigkeiten erneut ausschließlich in der Bestimmung der Anteile lagen. Die in den Analysen herausgestellten charakteristischen Fehler zeigen auf, dass die Lernenden versuchen das Verfahren auf die Zahlenwerte im Aufgabentext anzuwenden, dabei jedoch nicht erkennen, dass der vorgegebene Anteil von 34 Fahrrädern $\frac{2}{7}$ und nicht $\frac{1}{7}$ des Ganzen entspricht, und entsprechend in ihren Berechnungen die falschen Bezugsgrößen wählten. Die Analysen dokumentieren, dass die Lernenden in den meisten Fällen den Rechenweg fokussierten, ohne einen Bezug zum Aufgabenkontext herzustellen.

Verallgemeinerung des Handlungsschemas: Die Schwierigkeiten der Lernenden bei der Bestimmung von Anteilen kann im Rahmen der Verallgemeinerung des Verfahrens zur Anteilberechnung bzw. in der Verallgemeinerung des zugehörigen Handlungsschemas interpretiert werden. Im Vergleich der Bearbeitungen der unterschiedlichen Aufgaben konnte beobachtet werden, dass die Lernenden direkt nach dem Lesen der Aufgabenstellung versuchen eine Rechnung aufzustellen, ohne die Aufgabensituation in ihre Überlegungen einzubeziehen. In der Folge werden fehlerhafte Zuordnungen der Bezugsgrößen vorgenommen und die Notwendigkeit der Anpassung des Verfahrens wird in vielen Fällen erst erkannt oder in Betracht gezogen, wenn die naheliegenden Rechnungen nicht aufgehen. In den Argumentationen der Lernenden wird wiederholt geäußert, dass ,man das doch immer so“ rechne, weshalb dieses Fehlermuster als Fokussierung auf die Anwendung eines Handlungsschemas beschrieben werden kann. Diese Fokussierung auf die Anwendung eines Handlungsschemas führt dazu, dass das Hauptaugenmerk der Lernenden nicht auf der Verarbeitung der Aufgabensituation liegt, weshalb sie fehlerhafte Bezüge zwischen den im Aufgabentext enthaltenen Zahlenwerten und Größen herstellen.

Im Hinblick auf die Verallgemeinerung des Verfahrens konnten in den dargestellten Bearbeitungsprozessen verschiedene Fehlvorstellungen bzw. fehlerhafte Denkmuster dokumentiert werden. Diese umfassen im Allgemeinen eine komponentenweise Betrachtung von Zähler und Nenner eines Bruchs $\frac{m}{n}$, die in einem Fall dazu führte, dass die Teiloperatoren : $n$ und $\cdot m$ auf die falschen Bezugsgrößen angewendet wurde und das Ergebnis soweit korrigiert wurde, dass es plausibel erscheint. In einem anderen Fall wurde der Zähler des Bruchs $\frac{m}{n}$ nicht als Anzahl von Teilen eines Ganzen, sondern als absolute Anzahl von Fahrrädern interpretiert.

Die Ergebnisse der Analysen können im Allgemeinen so zusammengefasst werden, dass die Lernenden das Verfahren zur Anteilberechnung als Kalkül verstehen, das auf die vorgegebenen Zahlen und Größen angewendet wird. Es werden 
zunehmend weniger anschauliche Vorstellungen zu Anteilen aktiviert, auf deren Grundlage der Sachkontext strukturiert werden könnte. Diese Annahme wird insbesondere dadurch gestützt, dass die Lernenden zunehmend bis ausschließlich auf symbolischer Ebene argumentieren und arbeiten.

\subsection{Kürzen von Brüchen als Vergröbern der Einteilung}

In diesem Abschnitt werden ausgewählte Analysen der Daten aus der dritten und letzten videographierten Unterrichtssitzung dargestellt. In Folge auf die Thematisierung des Erweiterns von Brüchen als Verfeinern einer Einteilung wird in dieser Doppelstunde das Kürzen von Brüchen als Vergröbern einer Einteilung eingeführt. Im Folgenden werden zunächst die Lernmaterialien auf sachanalytischer Ebene dargestellt, die den Ausgangspunkt für die Übertragung des Verfahrens auf nachfolgende Transferaufgaben bilden.

Es ist zu bemerken, dass das Erweitern von Brüchen als Verfeinern einer Einteilung in der letzten Unterrichtsstunde vor der Datenerhebung eingeführt wurde. Die Einführung des Erweiterns wurde dabei im Klassenverbund anhand von strukturgleichen Lösungsbeispielen durch die Lehrperson vorgenommen. In dieser Stunde wurde lediglich das Verfahren eingeführt und an wenigen Aufgaben im Arbeitsbuch eingeübt.

Als Umkehrung zum Erweitern von Brüchen als Verfeinern einer Einteilung wird das Kürzen von Brüchen als Vergröbern einer Einteilung von ikonischen Bruchdarstellungen eingeführt. Den gemeinsamen Kern des Erweiterns und Kürzens von Brüchen stellt das Verständnis, dass ein Bruch $\frac{m}{n}$ durch Erweitern und Kürzen in unendlich viele äquivalente Bruchdarstellungen umgewandelt werden kann und somit unendlich viele Repräsentanten hat. Dieses Verständnis ist eine wesentliche Komponente von Grundvorstellungen und mentalen Modellen des Bruchzahlbegriffs (vgl. Ni, 2001, S. 412).

Lösungsbeispiel: Das Lösungsbeispiel besteht in drei analogen Teilen, in denen die Einteilung des Bruchs $\frac{12}{30}$ so verändert wird, dass halb so viele (Teil a), ein drittel so viele (Teil b) und ein sechstel so viele (Teil c) Teile entstehen. Der Ablauf der drei Teile des Lösungsbeispiels ist jeweils der gleiche (vgl. Abb. 5.36): Zunächst wird eine Rechteckdarstellung des Ausgangsbruchs $\frac{12}{30}$ aufgebaut und die Arbeitsanweisung ,Zeichne die neue Einteilung ein und gib einen Bruch dazu an. Überlege dann, wie man den neuen Bruch auch rechnerisch erhält" eingeblendet. 
Ein Rechteck ist in 30 Dreißigstel eingeteilt, 12 Dreißigstel davon sind grün gefärbt. Die Einteilung soll so geändert werden, dass man a) halb so viele, b) ein drittel so viele, c) ein sechstel so viele gleich große Teile erhält.

Zeichne die neue Einteilung ein und gib einen Bruch dazu an.

Öberlege dann, wie man den neuen Bruch auch rechnerisch erhält.

Lösung:

a) Wir vergröbern die Einteilung, so dass halb so viele Teile entstehen. Dann sind auch nur halb so viele Teile grün.

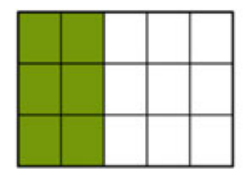

rechnerisch:

Zähler und Nenner werden durch 2 dividiert: $\frac{12}{30}=\frac{12: 2}{30: 2}=\frac{6}{15}$

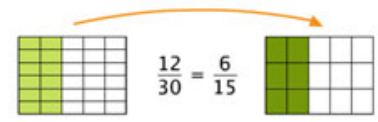

Abbildung 5.36 Endzustand des ersten Teils des animierten Lösungsbeispiels

Zur Lösung der Aufgabenstellung(en) wird zunächst beschrieben, dass die Einteilung der Bruchdarstellung so vergröbert wird, dass halb so viele Teile entstehen, wodurch in der neuen Darstellung des Bruchs auch nur halb so viele Teile grün markiert sind (vgl. 5.36). In der anschließenden Animation werden sukzessive zwei Teile der Einteilung zu einem Teil zusammengefasst, so dass die 30 Teile der Einteilung in 15 doppelt so großen Teilen zusammengefasst werden. Die abgeschlossene Animation wird schließlich durch eine Beschreibung des Rechenweges ergänzt, in der erläutert wird, dass das dargestellte Vergröbern der Einteilung rechnerisch dem Dividieren von Zähler und Nenner durch den Divisor 2 entspricht: $\frac{12}{30}=\frac{12: 2}{30: 2}=\frac{6}{15}$.

Der erste Teile des Lösungsbeispiels wird durch eine Gegenüberstellung der alten und neuen Einteilung des Bruches mitsamt der entsprechenden symbolischen Repräsentanten beendet, wobei ein Pfeil von der Anfangseinteilung zur Endeinteilung die Umwandlung herausstellt und ein Gleichheitszeichen zwischen den symbolischen Repräsentationen $\frac{12}{30}=\frac{6}{15}$ die Äquivalenz der beiden Brüche betont.

In den Arbeitsheften der Lernenden werden die statischen Abbildungen der Lösungsbeispiele durch jeweils eine fokussierende Fragestellung ergänzt:

Lösungsbeispiel Teil a): Was bedeutet es, eine Einteilung zu vergröbern? Was verändert sich, was bleibt gleich?

Lösungsbeispiel Teil b): Warum wird der Bruch nicht kleiner, obwohl man Zähler und Nenner durch 3 teilt? 
Lösungsbeispiel Teil c): Zähler und Nenner werden durch 6 geteilt. Was verändert sich im Rechteck, was bleibt gleich?

Die erste Frage fokussiert die Vergröberungshandlung auf ikonischer Ebene: Die Einteilung wird so verändert, dass je zwei Teile zu einem größeren Teil zusammengefasst werden. Dies führt dazu, dass die Anzahl der Teile halbiert, ihre Größe jedoch verdoppelt wird. Somit bleibt der Anteil der markierten Fläche an der ganzen Fläche invariant. Die fokussierende Fragestellung zum zweiten Teil des Lösungsbeispiels soll die Aufmerksamkeit auf die symbolische Handlung der Division von Zähler und Nenner mit demselben Faktor (Divisor) lenken. Es soll erläutert werden, dass bei der Division von Zähler und Nenner durch 3 der Anteil bzw. das Verhältnis von Zähler und Nenner nicht verändert wird. Die dritte fokussierende Fragestellung dient der Verknüpfung der Handlungen auf ikonischer und symbolischer Ebene. Es wird gefragt, was sich in der ikonischen Darstellung verändert, wenn der Zähler und der Nenner eines Bruchs durch denselben Faktor (Divisor) geteilt werden. Die Lernenden sollen dabei die Änderungen und Invarianzen auf den beiden Darstellungsebenen miteinander in Beziehung setzen. Auf ikonischer Ebene bewirkt die Division ein Zusammenfassen von sechs Teilen zu einem Teil, sodass die Anzahl der Teile gesechstelt wird. Der Anteil der gefärbten Fläche bleibt dabei jedoch unverändert, ebenso wie das Verhältnis von Zähler und Nenner.

Ausgewählte Transferaufgaben: Im Anschluss an die interaktiven animierten Lösungsbeispiele folgt eine Serie von Übungs- und Transferaufgaben (siehe Tab. 5.4). Detaillierte Aufgabenbeschreibungen und Erläuterungen der Transferprozesse erfolgen an den entsprechenden Stellen in diesem Abschnitt.

Tabelle 5.4 Aufgabensequenz - Anteile von beliebigen Größen

\begin{tabular}{l|l}
\hline Aufgabe & Transferprozesse \\
\hline 3.1 \& 3.2 & $\begin{array}{l}\text { Übertragung der Vergröberungshandlung und des Verfahrens } \\
\text { zum Kürzen von Brüchen aus dem Lösungsbeispiel auf } \\
\text { unvollständige Beispiele mit anderen Brüchen und anderen } \\
\text { Repräsentationsobjekten }\end{array}$ \\
\hline 3.3 & $\begin{array}{l}\text { Übertragung der Vergröberungshandlung von Rechteck- und } \\
\text { Kreisrepräsentationen auf eine Streckenrepräsentation }\end{array}$ \\
\hline
\end{tabular}




\subsubsection{Unvollständige Beispiele}

Im ersten unvollständigen Beispiel ist der Bruch $\frac{6}{18}$ in einer Rechteckrepräsentation dargestellt und die Einteilung soll so vergröbert werden, dass die neue Einteilung in Aufgabenteil a) halb so viele und in Aufgabenteil b) ein sechstel so viele Teile enthalten. In der ersten Teilaufgabe ist die Vergröberung der Einteilung bereits vorgegeben, sodass die Lernenden nur den gekürzten Bruch auf symbolischer Ebene ergänzen müssen: $\frac{6}{18}=\frac{3}{9}$. In Aufgabenteil b) sollen die Lernenden auch eine neue Einteilung in der Rechteckrepräsentation einzeichnen. Hierzu müssen sechs Teile des Ganzen zu einem Teil zusammengefasst werden, was zu einer Einteilung des Rechtecks in drei Teile führt, von denen ein Teil markiert wird. Auf symbolischer Ebene sollen die Lernenden sowohl den gekürzten Bruch $\frac{1}{3}$ ergänzen als auch die Beschreibung des Vorgehens „Man dividiert den Zähler und den Nenner des Bruchs durch 6“ und „Man nennt dies: Kürzen mit 6“ ergänzen. Als Hilfestellung markieren Sprechblasen die Arbeitsschritte, die von den Lernenden ergänzt bzw. durchgeführt werden sollen, z.B. „Ergänze die Rechnung“ in Teilaufgabe a) und „Zeichne eine neue Einteilung" in Aufgabenteil b) (Abb. 5.37).

Das Vorgehen im zweiten unvollständigen Beispiel orientiert sich erneut an den Bearbeitungsschritten des Lösungsbeispiels, nur ist hier der zu kürzende Bruch $\frac{6}{12}$ nicht in einem Rechteck, sondern in einem Kreis dargestellt. Analog zum Lösungsbeispiel soll die Einteilung so verändert werden, dass die neue Einteilung in Teilaufgabe a) halb so viele und in Teilaufgabe b) ein drittel so viele Teile hat als die Ausgangsdarstellung. Anders als im ersten unvollständigen Beispiel sind im zweiten Lösungsbeispiel keine Lösungsansätze vorgegeben, die von den Lernenden ergänzt werden sollen. Stattdessen sollen die Lernenden in beiden Teilaufgaben sowohl die neue Einteilung einzeichnen als auch den gekürzten Bruch angeben und das Vorgehen auf symbolischer Ebene jeweils in einem Satz beschreiben. Als Hilfestellung zur eigenständigen Durchführung des zeichnerischen Vergröberns der Einteilung und rechnerischen Kürzens des Bruchs werden in Sprechblasen Hinweise zum Vorgehen gegeben (Abb. 5.38).

Transferprozesse in den unvollständigen Beispielen: Die Transferprozesse zur Bearbeitung der unvollständigen Beispiele bestehen in der Übertragung und Anwendung des Verfahrens zum Vergröbern einer Einteilung auf ikonischer Ebene und des rechnerischen Verfahrens zum Kürzen von Brüchen auf symbolischer Ebene. Die Lernenden sind gefordert zunehmend selbstständig die beiden Verfahren auf neue Brüche und Bruchdarstellungen anzuwenden. Obwohl sich die Ausgangsbrüche jeweils von dem Bruch im Lösungsbeispiel unterscheiden, sind die Kürzungsfaktoren bzw. die Vorgaben zum Vergröbern der Einteilung im Lösungsbeispiel enthalten. 


\section{AUfGabe 1}

In einem Rechteck, in dem 6 Achtzehntel markiert sind, soll die Einteilung vergröbert werden.

Die neue Einteilung soll a) halb b) ein sechstel so viele Teile enthalten.

Teile das Rechteck neu ein. Gib außerdem einen Bruch für die neue Einteilung des Rechtecks an. Überlege dir auch, wie man rechnerisch auf den neuen Bruch kommt.

\section{Lösung:}

a) Man vergröbert die Einteilung des Rechtecks, so dass immer zwei Teile zusammengefasst werden und halb so viele Teile vorhanden sind.

Dann sind auch halb so viele Teile markiert.

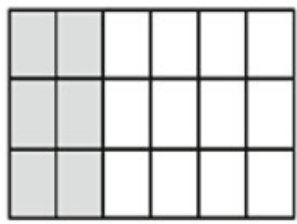

Rechnerisch:

Man dividiert Zähler und Nenner durch 2: Man nennt dies: Kürzen durch 2.

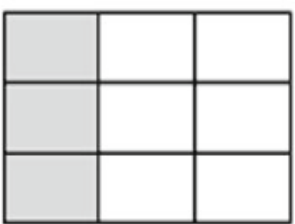

$\frac{6}{18}=$

b) Wir vergröbern die Einteilung, so dass ein sechstel so viele Teile entstehen.

Somit sind auch nur ein sechstel so viele Teile markiert.
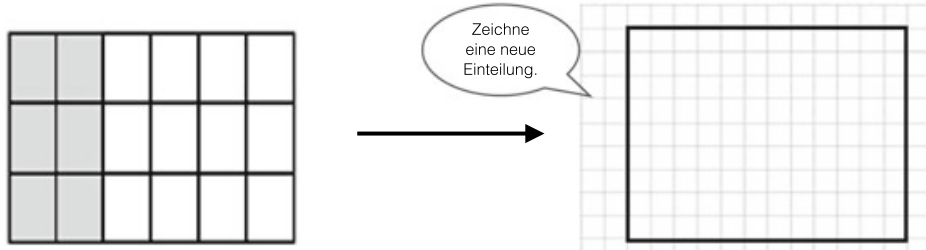

Rechnerisch:

Man dividiert...

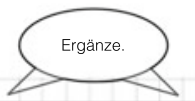

$\frac{6}{18}=$

Man nennt dies:

Kürzen durch ...

Abbildung 5.37 Erstes unvollständiges Beispiel 


\section{AUfGABE 2}

Ein Kreis ist in Zwölftel eingeteilt, davon sind 6 Zwölftel markiert.
Die neue Einteilung soll
a) halb
b) ein drittel
so viele Teile haben wie vorher.

Zeichne eine neue Einteilung in den Kreis und gib den Bruch für die neue Einteilung an. Wie erhält man den Bruch rechnerisch?

\section{Lösung:}

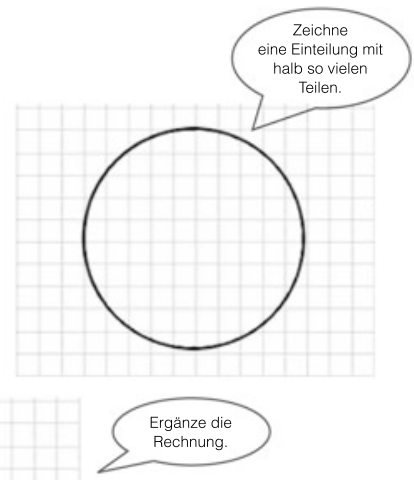

b)
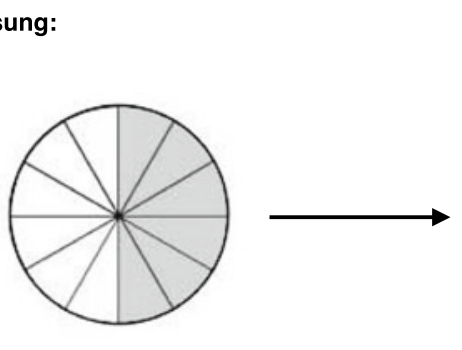

Rechnerisch: $\quad \frac{6}{12}=$
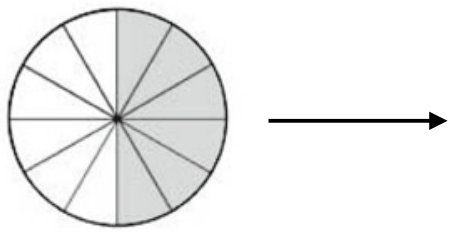

Rechnerisch:

$$
\frac{6}{12}=
$$

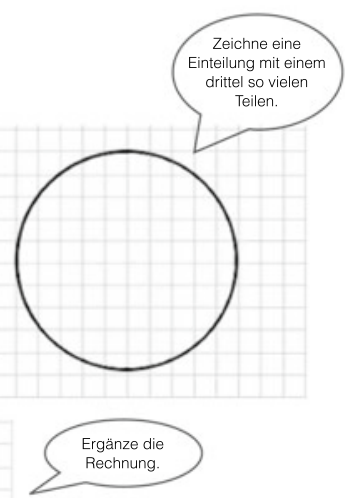

Abbildung 5.38 Zweites unvollständiges Beispiel

Das zweite unvollständige Beispiel enthält zudem einen Repräsentationstransfer. Im Gegensatz zum Lösungsbeispiel und dem ersten unvollständigen Beispiel ist der Anteil in einer Kreisrepräsentation und nicht wie zuvor in einem Rechteck dargestellt. 
Neben der Übertragung und Anwendung der Verfahren auf anschaulicher und symbolischer Ebene soll durch die Bearbeitung der unvollständigen Beispielen insbesondere die Verknüpfung der Handlungen auf anschaulicher und symbolischer Ebene mit dem Ziel der Ausbildung der Grundvorstellung vom Kürzen als Vergröbern einer Einteilung unterstützt werden. Vor diesem Hintergrund wird die Anwendung der Verfahren durch Bearbeitungshinweise in Sprechblasen angeleitet, um die kognitive Belastung der Lernenden möglichst gering zu halten.

\section{Bennet \& Julius - Übertragung des rechnerischen Verfahrens}

Bennet und Julius lesen alle Teile des Lösungsbeispiels eingehend und diskutieren ausführlich die Antworten auf die fokussierenden Fragestellungen. In ihren Erklärungen sind die zentralen Aspekte des Vergröberns einer Einteilung deutlich zu erkennen. Auf die erste fokussierende Fragestellung, in der gefragt wird, was es bedeutet, eine Einteilung zu vergröbern, notieren sie: „Man macht größere Stücke aus den kleineren. Die Stücke werden größer, dafür sind es weniger. Das grüne [farbig markierte] Feld bleibt gleich groß“ (vgl. Abb. 5.39). Die folgenden Transkriptsequenzen dokumentieren ihre Bearbeitung der unvollständigen Beispiele.

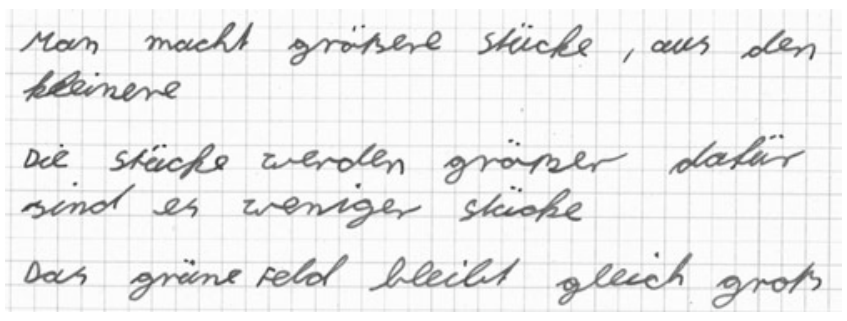

Abbildung 5.39 Julius Antwort auf die erste fokussierende Fragestellung nach der Bedeutung des Vergröberns einer Einteilung

\section{Transkript B3A1 - Bennet \& Julius - Szene 1 - Aufgabe 1 a)}

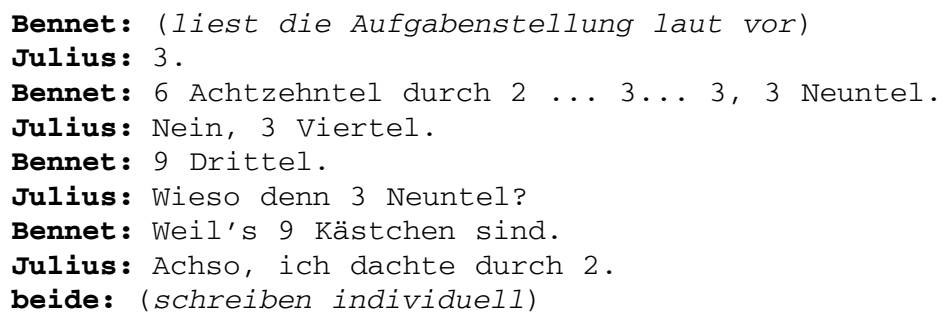


In der Bearbeitung von Teilaufgabe a) ist zu erkennen, dass Bennet und Julius unterschiedlich vorgehen. Bennet betrachtet die vorgegebene Rechteckdarstellung mit der neuen Einteilung, zählt die Kästchen in Dreierschritten und liest schließlich den neuen Bruch $\frac{3}{9}$ ab (3). Anders als sein Partner Bennet nutzt Julius die Darstellung nicht, sondern versucht auf Zahlenebene den Zähler und Nenner von $\frac{6}{18}$ durch 2 zu teilen. Dabei passiert ihm ein Fehler beim Teilen des Nenners und statt $18: 2$ rechnet er $8: 2$ und kommt so auf den neuen Bruch $\frac{3}{4}(2,4)$. Aufgrund der unterschiedlichen Ergebnisse widerspricht Julius seinem Partner und fragt ihn, „wieso denn 3 Neuntel“ (6) richtig sei, worauf Bennet ihm antwortet, dass die neue Einteilung aus „9 Kästchen bestehe“ (7).

Julius erkennt seinen Rechenfehler nicht und kommt schließlich zu dem Schluss, dass die beiden Lösungswege nicht mit einander zusammenhängen. Die Begründung von Bennet kommentiert er mit „Achso, ich dachte durch 2“ (8) und scheint die Richtigkeit seines Lösungsweges, der Division von Zähler und Nenner durch 2, in Frage zu stellen.

Die unterschiedlichen Lösungswege von Bennet und Julius können so interpretiert werden, dass sie jeweils die für sie vertrauteren Zugänge zur Bearbeitung wählen. Bennet wählt einen Lösungsweg auf anschaulicher Ebene und liest den neuen Bruch aus der vorgegebenen Darstellung ab. Im Gegensatz dazu wählt Julius einen Lösungsweg auf Zahlenebene und scheint die ikonischen Bruchdarstellungen nicht für seine Lösung zu nutzen.

\section{Transkript B3A1 - Bennet \& Julius - Szene 2 - Aufgabe 1 b)}

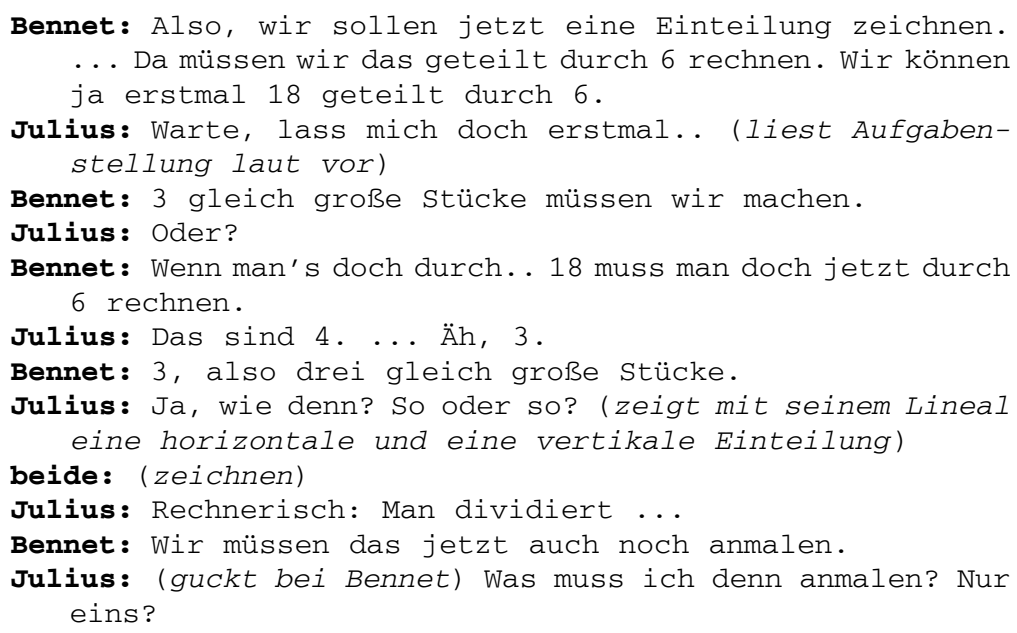




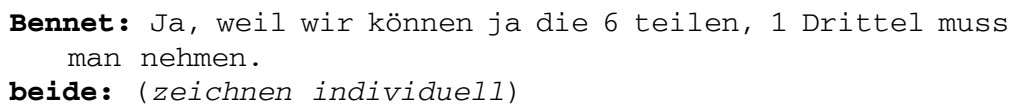

In der Bearbeitung von Teilaufgabe b) soll die Einteilung so vergröbert werden, dass ein sechstel so viele Teile entstehen wie zuvor. Bennet sagt, dass sie der Aufgabenstellung entsprechend ,jetzt eine Einteilung zeichnen“ (10) sollen. Er erklärt, dass man dazu ,geteilt durch 6 rechnen“ (11) und in der Folge „drei gleich große Stücke“ (15) einzeichnen müsse. Julius bittet Bennet zu Beginn kurz zu warten, da er ,doch erstmal“ (13) die Aufgabenstellung lesen möchte. Nachdem er die Aufgabenstellung laut vorgelesen hat, nimmt er den Lösungsweg von Bennet auf, dividiert 18 durch sechs: „Das sind 4. ... Äh 3“ (19). Bennet stimmt ihm zu und übersetzt das Ergebnis dieser Rechnung in die ikonische Ebene: „3, also drei gleich große Stücke“ (20).

Bennet und Julius bestimmen die neue Einteilung des Ganzen rechnerisch. Dazu wechseln sie auf die symbolische Ebene und berechnen die neue Einteilung über die Division 18 : 6, deren Ergebnis sie auf ikonischer Ebene als drei Teile interpretieren. In ihrer Lösung wechseln sie mehrfach zwischen der ikonischen und symbolischen Darstellungsebene. Es ist jedoch nicht zu erkennen, dass sie mit ihrem Vorgehen das Handlungskonzept des Vergröberns der Einteilung als Zusammenfassen von Teilen verbinden. Stattdessen berechnen sie eine neue Einteilung und zeichnen diese ein, ohne dabei einen direkten Zusammenhang zu der ursprünglichen Einteilung bzw. Darstellung herzustellen. Diese Annahme wird durch die Nachfrage von Julius unterstützt, der seinen Partner fragt, ob sie die neue Einteilung horizontal oder vertikal einzeichnen sollen (21-22). Ihr Lösungsweg beschreibt somit keine Transformation der ursprünglichen Darstellung, sondern das Herstellen einer neuen Darstellung.

In der Interaktion von Bennet und Julius fällt auf, dass die Übersetzungen zwischen der ikonischen und symbolischen Darstellungsebene alle von Bennet ausgehen und alle Beiträge von Julius die symbolischer Ebene betreffen. Zudem wirkt er überrascht davon, dass Bennet nur einen der drei Teile gefärbt hat: „Was muss ich denn anmalen? Nur eins?“ (26). Diese Beobachtungen sprechen dafür, dass er vordergründig die symbolische Handlung überträgt und diese nicht mit der Handlung auf ikonischer Ebene verbindet. Auch Bennets Erklärung bezieht sich allein auf die symbolische Handlung des Kürzens und er erklärt, dass sie ,ja die 6 teilen“ und „1 Drittel [...] nehmen“ (27-28) müssen. 
a) Man vergröbert die Einteilung des Rechtecks, so dass immer zwei Teile zusammengefasst werden und halb so viele Teile vorhanden sind.

Dann sind auch halb so viele Teile markiert.

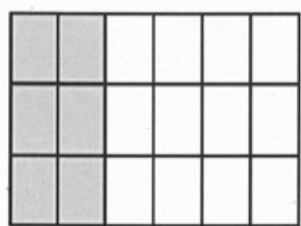

Rechnerisch:

Man dividiert Zăhler und Nenner durch 2:

Man nennt dies: Kürzen durch 2.

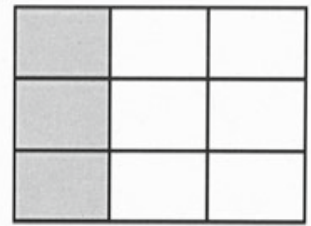

$\frac{6}{18} \stackrel{2}{=} \frac{3}{9}$

b) Wir vergröbern die Einteilung, so dass ein sechstel so viele Teile entstehen.

Somit sind auch nur ein sechstel so viele Teile markiert.
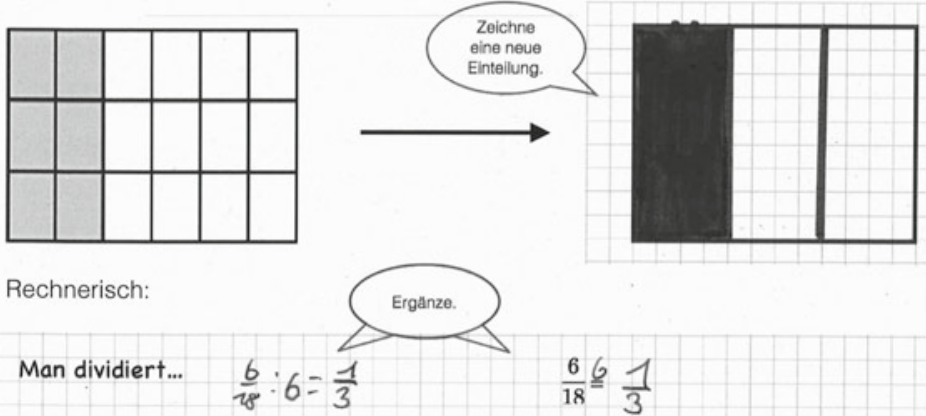

Man nennt dies:

Kürzen durch ... echte Bräche erwoitern

Abbildung 5.40 Bennets Lösung von Aufgabe 3.1

\section{Transkript B3A1 - Bennet \& Julius - Szene 3 - Aufgabe 1 b)}

Julius: Also, rechnerisch. Man dividiert ... man dividiert ... 6 Achtel durch 2 .

Bennet: Ja.

Julius: Durch 2 .

Bennet: Nein, durch 6.

Julius: Nicht durch 2? Warum denn nicht?

Bennet: Das ist doch geteilt durch 6 .

Julius: Aber man kann doch auch durch 2 rechnen, oder nicht?

Bennet: Ja nö. 


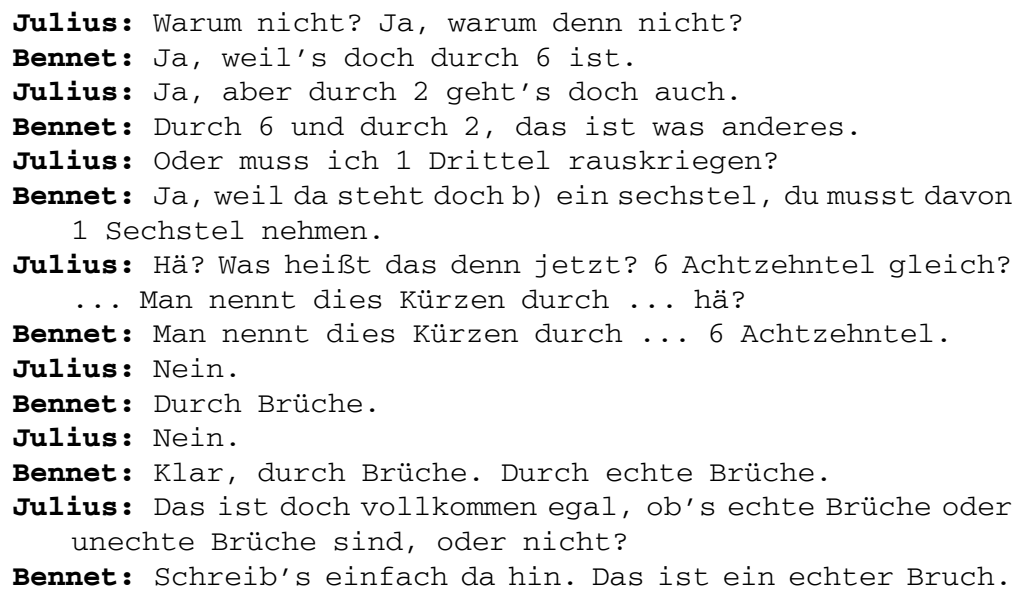

Die dritte Szene der Bearbeitung des ersten unvollständigen Beispiels dokumentiert die Formulierung des rechnerischen Vorgehens zum Kürzen von $\frac{6}{18}$. Hiebei sind deutlich die Verständnisschwierigkeiten von Julius zu erkennen. Er greift die Formulierung aus Aufgabenteil a) auf und schlägt vor zu schreiben ,,man dividiert ...6 Achtel durch 2" (38-39). Den Widerspruch von Bennet (42) scheint er nicht nachvollziehen zu können und er erwidert, dass man ,,doch auch durch 2 rechnen“ (45 und 49) könne. Auf die Erklärung von Bennet, dass ,durch 6 und durch 2 [...] was anderes“ (50) sei, fragt Julius, ob man „1 Drittel rauskriegen“ müsse (51). Scheinbar versteht Julius nicht, dass ein Bruch mit verschiedenen Faktoren gekürzt werden kann. Es ist anzunehmen, dass diese Verständnisschwierigkeit von Julius darauf zurückgeführt werden kann, dass er das rechnerische Kürzen von Brüchen nicht mit der Handlung des Vergröberns einer Einteilung verbindet und entsprechend keine anschaulichen Vorstellungen aktivieren kann. Alternativ ist es möglich, dass er das Kürzen von Brüchen mit 2 übergeneralisiert und annimmt, dass das Kürzen von Brüchen immer mit dem Faktor 2 durchgeführt wird. Zudem erkennt er scheinbar keinen Unterschied zwischen dem Kürzen mit 2 und dem Kürzen mit 6.

Auch Bennet hat Schwierigkeiten mit der Formulierung einer Beschreibung des rechnerischen Vorgehens und weiß nicht, was sie an der entsprechenden Stelle im Arbeitsheft notieren sollen. Ohne sich an der vorgegebenen Formulierung in Aufgabenteil a) zu orientieren notiert er schließlich zwei Gleichungen: , $\frac{6}{18}: 6=\frac{1}{3}$ “ (siehe Abb.5.40), womit er vermutlich ausdrücken will, dass der Zähler und der Nenner durch 6 geteilt werden sollen. Als zweite symbolische Beschreibung überträgt er die beim Erweitern von Brüchen durch den Lehrer eingeführte Schreibweise 
und notiert , $\frac{6}{18} \stackrel{6}{=} \frac{1}{3}$ “ (vgl. Abb. 5.40). Direkt im Anschluss gehen Bennet und Julius zur Bearbeitung des zweiten unvollständigen Beispiels über.

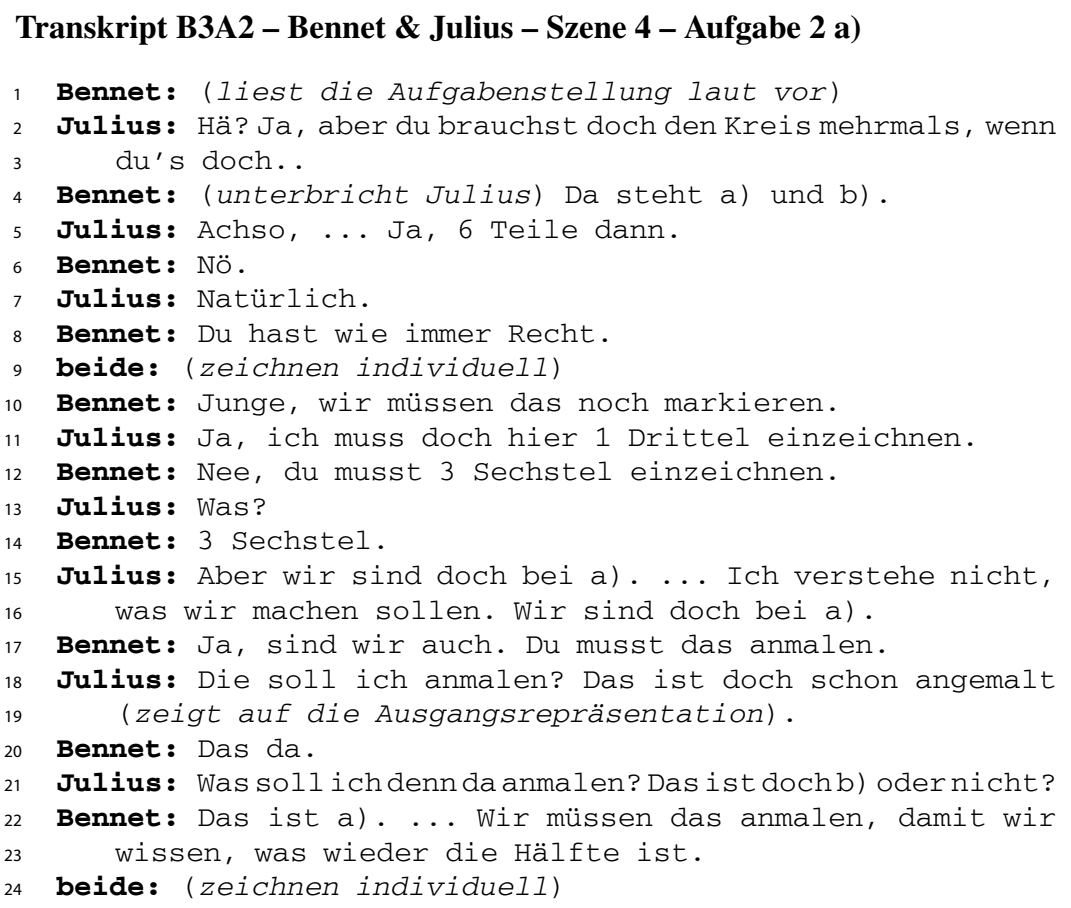

Nachdem Bennet und Julius Unstimmigkeiten bezüglich des Aufgabenlayouts geklärt haben, sagt Julius, dass die neue Einteilung aus ,6 Teile[n]“ (5) bestehen muss, und die beiden Partner zeichnen die neue Einteilung individuell ein. Im Anschluss an das Zeichnen der neuen Einteilung bemerkt Bennet, dass sie noch den Anteil markieren müssen, worauf Julius erwidert, dass man ,,doch hier 1 Drittel einzeichnen“ (11) müsse. Bennet widerspricht diesem Vorschlag und korrigiert, dass „3 Sechstel“ (12) markiert werden müssen. Seinem Vorschlag zu Folge versteht Julius das Einzeichnen des Anteils als Einzeichnen eines neuen Bruchs. Anstatt den äquivalenten Anteil zur Ausgangsdarstellung einzuzeichnen, möchte er $\frac{1}{3}$ einzeichnen, da eine Einteilung mit einem drittel so vielen Teilen eingezeichnet werden soll. Somit steht die neue Darstellung für ihn in keinem Zusammenhang zu der Ausgangsdarstellung und er interpretiert das Vorgehen entsprechend nicht als Ver- 
gröbern der Einteilung und Markieren desselben Anteils, sondern als Einzeichnen einer neuen Einteilung und Einzeichnen eines neuen Anteils.

Julius' Verständnisschwierigkeiten zeigen sich insbesondere in seiner Reaktion auf die Erwiderung von Bennet, dass sie nicht $\frac{1}{3}$, sondern „3 Sechstel einzeichnen“ $(12,14)$ sollen. Er sagt zunächst, dass er nicht verstehe, was sie machen sollen und ist zudem davon irritiert, dass sie denselben Anteil erneut einzeichnen sollen, da dieser in der Ausgangsdarstellung „,doch schon angemalt“ (18) sei. Bennet erklärt: „Wir müssen das anmalen, damit wir wissen, was wieder die Hälfte ist“ (23-24), was darauf hindeutet, dass er im Gegensatz zu Julius bereits verstanden hat, dass beide Darstellungen denselben Anteil in unterschiedlichen Darstellungen repräsentieren. Insbesondere spricht er dabei von „der Hälfte“ (24), womit er den vollständigen gekürzten Bruch für seine Erklärung nutzt, der im Aufgabenmaterial nicht benannt wird.

\section{Transkript B3A2 - Bennet \& Julius - Szene 5 - Aufgabe 2 a)}

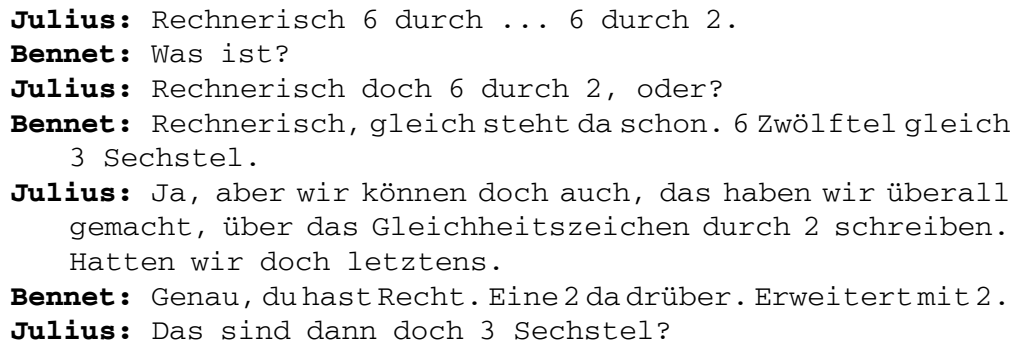

Nach dem Einzeichnen der vergröberten Bruchdarstellung notieren Bennet und Julius den gekürzten Bruch. Dabei orientiert sich Julius an ihrer Notation im ersten unvollständigen Beispiel (Abb. 5.41), in dem auch die Rechnung zum Kürzen des Bruchs notiert werden sollte. Er schlägt demnach vor aufzuschreiben, dass „6 durch 2 " $(26,28)$ gerechnet werden müsse. Bennet erwidert, dass bereits ein Gleichheitszeichen vorgegeben sei und sie daher nur den gekürzten Bruch notieren sollen: „6

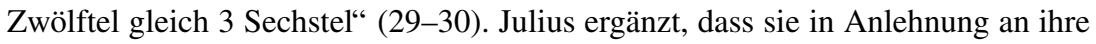
Notation beim Erweitern von Brüchen ,über das Gleichheitszeichen durch 2 schreiben“ (31-33) sollten, da sie das ,überall gemacht“ (ebd.) hätten. Es ist zu erkennen, dass die Schreibweise des Erweiterns auf das Kürzen von Brüchen übertragen wird, wobei Bennet sogar die Bezeichnung des Verfahrens überträgt und die Notation mit „erweitert mit 2“ (34-35) übersetzt. 
a)
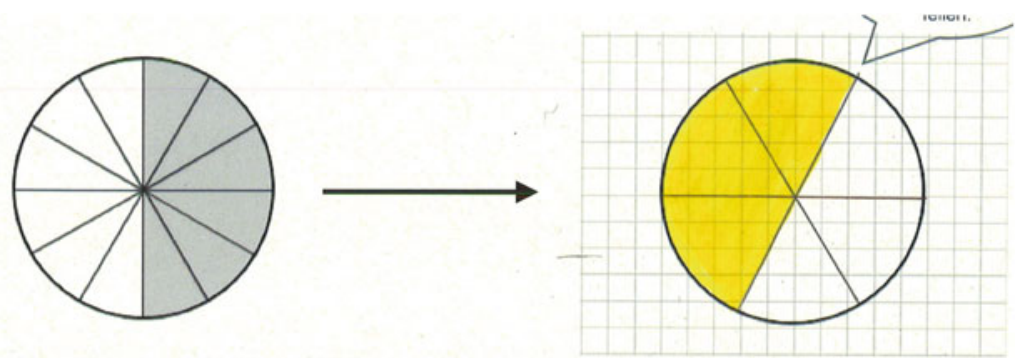

Rechnerisch: $\quad \frac{6}{12} \stackrel{2}{=} \frac{3}{6}$

b)
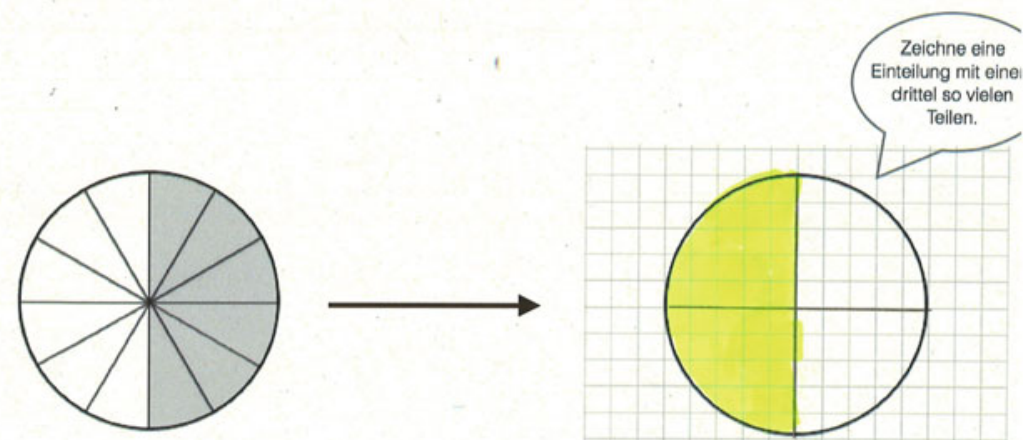
Teilen.

Rechnerisch: $\quad \frac{6}{12} \stackrel{3}{=} \frac{2}{4}$

Abbildung 5.41 Julius Lösung von Aufgabe 3.2

\section{Transkript B3A2 - Bennet \& Julius - Szene 6 - Aufgabe 2 b)}

Bennet: So, jetzt müssen wir das da einzeichnen. Geteilt durch 3 .

Julius: Das sind schon wieder 12 .

Bennet: Jetzt müssen wir das so teilen.

Julius: Nein, 4 ist falsch.

Bennet: Warum denn? 12 geteilt durch 3 gleich (schnalzt mit der Zunge). 


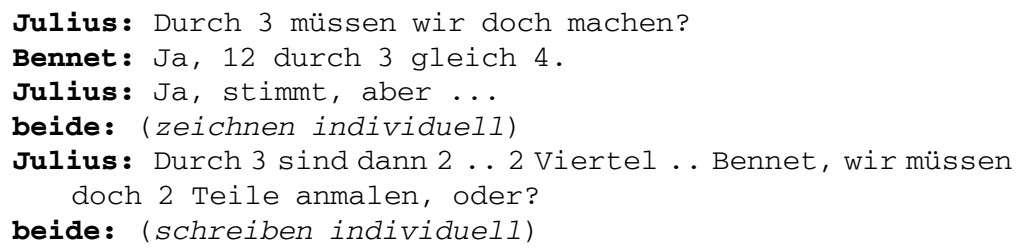

Unmittelbar zu Beginn der Bearbeitung von Aufgabenteil b), in dem die Einteilung des Bruchs $\frac{6}{12}$ so vergröbert werden soll, dass die neue Einteilung ein drittel so viele Teile hat wie die ursprüngliche Einteilung, äußert Julius sein Unverständnis, dass die ursprüngliche Einteilung erneut aus zwölf Teilen besteht (39). Seine geäuBerten Verständnisschwierigkeiten stützen die zuvor beschriebene Vermutung, dass er nicht erkennt, dass ein Bruch durch Kürzen in verschiedene wertgleiche Brüche umgewandelt werden kann.

Julius widerspricht neben dem anfänglichen Vorschlag von Bennet, dass sie für die neue Einteilung bzw. den neuen Bruch ,geteilt durch 3“ (37-38) rechnen müssen, auch der Zeichnung von Bennet, der die vorgegebene Kreisrepräsentation unterdessen in vier gleiche Teile eingeteilt hat. Er sagt: „Nein, 4 ist falsch“ (41), da sie durch 3 rechnen müssten (44). Bennet erklärt ihm, dass sie „,12 geteilt durch 3“ (42-43) rechnen müssen, was schließlich vier sei und die neue Einteilung entsprechend aus vier Teilen bestehe. Julius erkennt seinen Rechenfehler, stimmt Bennet zu (46) und die beiden Schüler beginnen eine neue Einteilung zu zeichnen. Zuletzt sucht Julius Bestätigung bei seinem Partner, dass er alles richtig gemacht hat und erklärt: „Durch 3 sind dann 2 ... 2 Viertel [...] wir müssen doch 2 Teile anmalen, oder?“ (48-49). Diese Erklärung deutet darauf hin, dass er wie in den vorherigen Bearbeitungen zunächst den Bruch rechnerisch gekürzt hat und sein Ergebnis als neuen Bruch interpretiert, den er in der vorgegebenen Kreisrepräsentation einzeichnen soll.

Insgesamt wird in Bennet und Julius' Bearbeitung der unvollständigen Beispiele deutlich, dass sie ausschließlich das Verfahren des Kürzens von Brüchen auf symbolischer Ebene übertragen und anwenden. Den rechnerisch erhaltenen gekürzten Bruch stellen sie in der Folge zeichnerisch dar. Es ist nicht zu erkennen, dass sie das rechnerische Kürzen als Transformation der Einteilung einer Bruchdarstellung interpretieren und diese entsprechend nicht als eine dynamische Handlung des Zusammenfassens von Teilen bzw. Vergröberns der Einteilung verstehen.

Insgesamt kann die Analyse der Bearbeitungsprozesse von Bennet und Julius in folgenden Deutungshypothesen zusammengefasst werden:

- Das Kürzen von Brüchen wird nicht mit dem Vergröbern einer Einteilung verbunden. Bennet und Julius übertragen vorwiegend das rechnerische Verfahren 
zum Kürzen von Brüchen als Division von Zähler und Nenner durch denselben Kürzungsfaktor. Das Handlungsschema des Vergröberns einer Einteilung wird nicht übertragen und angewendet. In der Folge besteht ihr Vorgehen in zwei Schritten: Im ersten Schritt berechnen sie auf symbolischer Ebene den neuen Bruch, den sie im zweiten Schritt ikonisch darstellen. In ihren Bearbeitungsprozessen ist nicht zu erkennen, dass sie die rechnerische Handlung des Kürzens mit der anschaulichen Vorstellung des Vergröberns einer Einteilung verbinden.

- Die Bruchdarstellungen von Ausgangsbruch und gekürztem Bruch werden als getrennte Entitäten aufgefasst. Obgleich Bennet und Julius erkennen, dass in beiden Bruchdarstellungen der gleiche Anteile gefärbt wird, stellen sie keinen dynamischen Zusammenhang - im Sinne einer Transformation der Einteilung zwischen den beiden Bruchdarstellungen her. Es ist möglich, dass die getrennte Betrachtung der beiden Bruchdarstellungen durch die Formulierung in der Aufgabenstellung ,zeichne eine neue Einteilung“ unterstützt wird, da diese möglicherweise als Einzeichnen eines „,neuen“ Bruchs interpretiert werden könnte.

- Aufgrund mangelnder anschaulicher Vorstellungen werden fehlerhafte Assoziationen hergestellt. In der Bearbeitung des zweiten unvollständigen Beispiels interpretiert Julius die Aufgabenstellung eine neue Einteilung mit einem drittel so vielen Teilen einzuzeichnen mit dem Einzeichnen des Bruchs $\frac{1}{3}$. Hierbei wird deutlich, dass die Formulierung in der Aufgabenstellung nicht auf die Einteilung, sondern auf den Bruch bezogen wird, was wiederum darauf zurückgeführt werden kann, dass mit dem Kürzen von Brüchen keine dynamische Handlung als Vergröbern der Einteilung verbunden wird.

\section{Anita \& Ann-Katrin - Trennung von ikonischer und symbolischer Ebene}

Anita und Ann-Katrin arbeiten harmonisch und gewissenhaft zusammen. In ihrer Partnerarbeit sprechen sie ausführlich über ihre Lösungswege und klären Verständnisschwierigkeiten gemeinsam auf. Unabhängig von ihren heterogenen Vortestergebnissen von 30\% (Ann-Katrin) und 70\% (Anita) konnten sie bisher alle Aufgaben korrekt lösen. Der Vergleich der Vor- und Nachtestergebnisse dokumentiert, dass Ann-Katrin über den Verlauf der Unterrichtseinheit einen weit überdurchschnittlichen Leistungszuwachs erzielt und im Posttest ein Ergebnis von 85\% erreicht.

Im Einstieg in diese Unterrichtsstunde war zu beobachten, dass sie alle Teile des Lösungsbeispiels eingehend gelesen und sich die jeweiligen Lösungsschritte gegenseitig erklärt haben. Auch ihre Antworten auf die fokussierenden Fragestellungen zum Lösungsbeispiel deuten darauf hin, dass sie die wesentlichen Aspekte des Kürzens von Brüchen als Vergröbern einer Einteilung erfasst haben (vgl. Abb. 5.42). Die folgenden Transkripte dokumentieren ihre Bearbeitungen der unvollständigen Beispiele. 


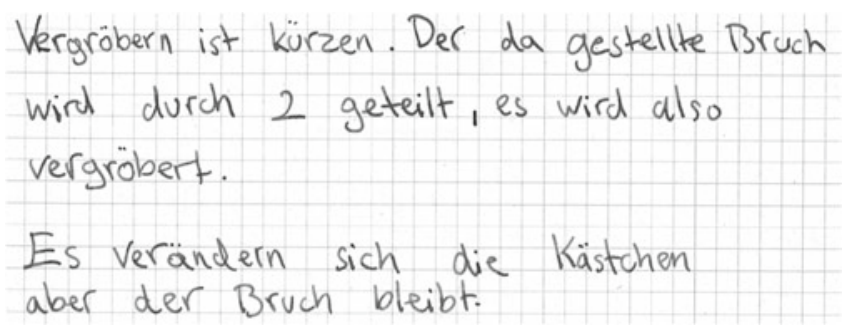

Abbildung 5.42 Anitas Antwort auf die fokussierende Fragestellung zum ersten Teil des Lösungsbeispiels

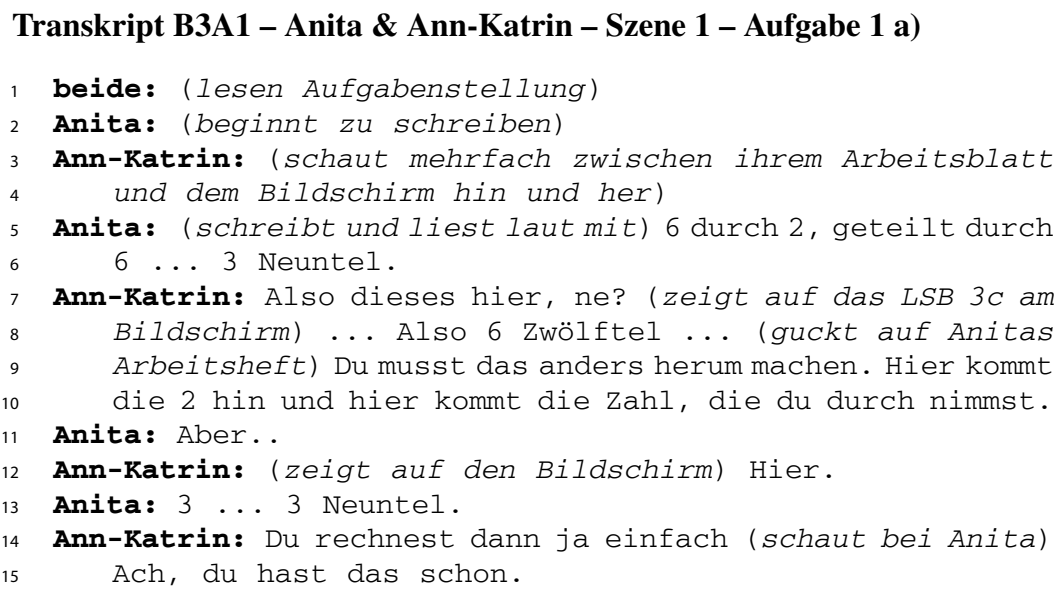

Die beiden Schülerinnen lesen die Aufgabenstellung still für sich selbst. Unmittelbar nach dem Lesen der Aufgabenstellung beginnt Anita zu schreiben, während Ann-Katrin mehrfach zwischen dem Endzustand des dritten Teils des Lösungsbeispiels auf dem Bildschirm und der Aufgabenstellung hin und her schaut (3-4). Augenscheinlich vergleicht sie die Aufgabenstellungen und versucht die einzelnen Lösungsschritte zu übertragen.

Währenddessen schreibt Anita in ihrem Arbeitsheft und liest laut mit: „6 durch 2, geteilt durch 6 ... 3 Neuntel“ (5-6). Ihre schriftlichen Dokumente (vgl. Abb. 5.43) bestätigen, dass sie schreibt: , $\frac{6}{18}=\frac{2: 6}{6: 18}=\frac{3}{9}$ “ und demnach das Verfahren zum Kürzen von Brüchen, unabhängig von ihren Vertauschungen von Dividenden und Divisoren, auf den neuen Bruch überträgt und anwendet. 


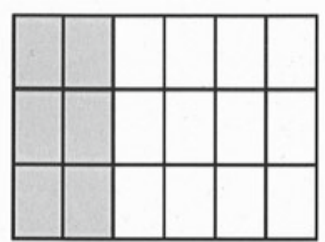

Rechnerisch:

Man dividiert Zähler und Nenner durch 2:

Man nennt dies: Kürzen durch 2.

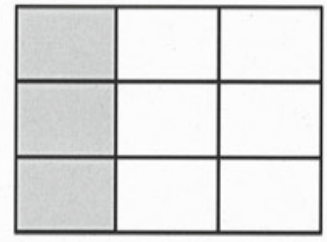

$$
\frac{6}{18}=\frac{2: 6}{2: 18}=\frac{3}{9}
$$

Abbildung 5.43 Anitas Lösung von Aufgabe 3.1 a)

Ann-Katrin korrigiert den aufgeschriebenen Term ihrer Partnerin mit Verweis auf die Rechnung im Lösungsbeispiel am Bildschirm und setzt schließlich zu einer eigenen Formulierung der Rechnung an. Diese führt sie jedoch nicht zu Ende, da sie feststellt, dass Anita bereits mit der Teilaufgabe fertig ist. Die ikonische Darstellung der neuen Einteilung wird in der Kommunikation der beiden Schülerinnen nicht angesprochen.

\section{Transkript B3A1 - Anita \& Ann-Katrin - Szene 2 - Aufgabe 1 b)}

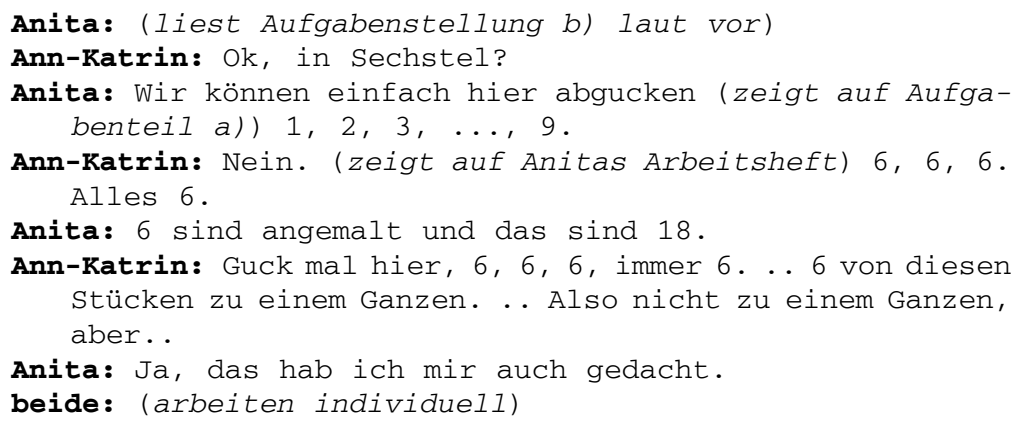

Für die Bearbeitung von Aufgabenteil b) liest Anita die Aufgabenstellung zunächst laut vor und macht dann den Vorschlag, dass sie ja „einfach hier abgucken“ (18) könnten, wobei sie auf den Aufgabenteil a) zeigt und die Anzahl der Teile der Einteilung auszählt (19). Es ist anzunehmen, dass sie weiß, dass sie das Verfahren im Allgemeinen übertragen kann, dabei jedoch nicht bedenkt, dass in der Aufgaben- 
stellung das Zeichnen einer neuen Einteilung mit einem sechstel so vielen Teilen und nicht mit halb so vielen Teilen wie in Teilaufgabe a) gefordert wird.

Ann-Katrin widerspricht diesem Vorschlag umgehend, beschreibt die Ausgangsrepräsentation: „,6, 6, 6. Alles 6“ (20-21) und erklärt im Weiteren: „Guck mal hier, 6, 6,6 , immer 6. ... 6 von diesen Stücke zu einem Ganzen“ (23-24), wobei sie deutlich macht, dass sie jeweils sechs Teile der Einteilung zu einem Teil zusammenfassen möchte. Sie präzisiert weiter, dass sie die sechs Teile „nicht zu einem Ganzen, aber“ (24-25) zu einem Teil zusammenfassen möchte. Anita stimmt ihr zu sagt, dass sie sich das ,auch gedacht“ (26) habe. In der Folge zeichnen die beiden Schülerinnen individuell eine neue Einteilung und schreiben den Rechenweg auf, wobei Anita die Vertauschung von Dividend und Divisor aus Aufgabenteil a) wiederholt schreibt: , $\frac{6}{18}=\frac{6: 6}{6: 18}=\frac{1}{3}$ “ (siehe Abb. 5.44).

Wir vergröbern die Einteilung, so dass ein sechstel so viele Teile entstehen.

Somit sind auch. nur ein sechstel so viele Teile markiert.
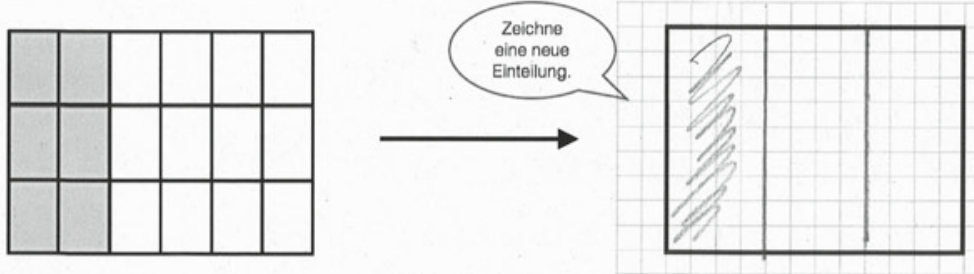

Rechnerisch:

Man dividiert...

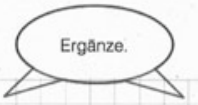

$$
\frac{6}{18}=\frac{6: 6}{6: 18}=3
$$

Man nennt dies:

Kürzen durch ... 6

Abbildung 5.44 Anitas Lösung von Aufgabe 3.1 b)

In dieser Bearbeitung wird deutlich, dass Ann-Katrin das Vergröbern einer Einteilung auf ikonischer Ebene mit dem Zusammenfassen von Teilen interpretiert und entsprechend das Handlungsmuster aus dem Lösungsbeispiel auf eine neue Bruchdarstellung übertragen und anwenden kann. Ihre Erklärung deutet zudem darauf hin, dass sie sich das Zusammenfassen von Teilen dynamisch vorstellt. Sie korrigiert sogar ihre Formulierung, dass „6 von diesen Stücken zu einem Ganzen“ (23-24) 
zusammengefasst werden und erklärt, dass die Teile nicht zu einem Ganzen, sondern einem neuen Teil zusammengefasst werden.

\section{Transkript B3A2 - Anita \& Ann-Katrin - Szene 3 - Aufgabe 2 a)}

Anita: Geteilt durch 2. (beginnt zu schreiben)

Ann-Katrin: (blättert in ihrem Arbeitsheft zurück) Ah, halb so viele. Also das jetzt noch mal halb machen. Das hier alles nochmal durch 2 teilen.

beide: (zeichnen individuell)

Ann-Katrin: Aah, 3 Sechstel. (schaut bei Anita) Stopp, 3 Sechstel hier. 1, 2, 3, 3, 3, dann sind as aber ...

Anita: $1,2,3, \ldots, 12$.

Ann-Katrin: Ich verstehe das gerade alles nicht.

Anita: Ich schon. (beginnt zu zeichnen)

Ann-Katrin: Ich weiß nicht, wie man das machen soll. (guckt bei Anita) Ich verstehe das nicht. ... (klickt die LSBe am Bildschirm durch) Gibt's da einen Kreis? (guckt erneut bei Anita) Warte, wo ist da der Nenner und wo ist der Zähler?

Anita: Zähler ist oben, Nenner ist unten.

Ann-Katrin: Ok, also das dann in 6 Teile teilen, oder wie? Anita: 3 davon.

Ann-Katrin: Boah ey, ich verstehe echt nichts mehr. Das ist echt das schwierigste. Die anderen Aufgaben waren besser.

beide: (schreiben/zeichnen individuell)

Anita: 1, 2, 3, 4, 5, 6. Ich hab gerade 6 stücke gemacht. ... Fertig.

Ann-Katrin: Ich verstehe überhaupt nichts. Wie hast du das geschafft?

Anita: Ich hab von hier hin, bis da wo es ist.

Ann-Katrin: (beginnt zu zeichnen)

beide: (schreiben/zeichnen individuell)

Anita: Ich hab's richtig. ...2 Viertel, das ist ganz einfach. (guckt bei Ann-Katrin) Du hast das ja perfekter gemacht als ich.

Ann-Katrin: Ja, ich hab das vorher schon berechnet. So, los komm, mach 2 stücke.

beide: (schreiben/zeichnen individuell) 
Die Bearbeitung des zweiten unvollständigen Beispiels erfordert einen Repräsentationstransfer. Im Gegensatz zu den ikonischen Bruchdarstellungen im Lösungsbeispiel und dem ersten unvollständigen Beispiel, in denen Brüche ausschließlich in einem Rechteck repräsentiert werden, ist der Bruch $\frac{6}{12}$ in einer Kreisrepräsentation vorgegeben. Obwohl Ann-Katrin in der Bearbeitung des ersten unvollständigen Beispiels das Vergröbern einer Einteilung als Zusammenfassen von Teilen zu einem größeren Teil beschrieben hat und auch das Verfahren zum Kürzen von Brüchen auf symbolischer Repräsentationsebene übertragen und angewendet hat, bereitet ihr die Anwendung des Verfahrens auf eine Kreisrepräsentation Schwierigkeiten. Sie erkennt genau wie Anita, dass sie für eine Einteilung mit halb so vielen Teilen ,geteilt durch 2“ (1) rechnen müssen und erklärt: „Ah, halb so viele. Also das jetzt noch mal halb machen. Das hier alles nochmal durch 2 teilen“ (2-4). Anhand der Videoaufzeichnung ist nicht eindeutig zu erkennen, ob sie sich mit ihrer Beschreibung des Vorgehens auf die ikonische oder die symbolische Bruchdarstellung bezieht. Ihre Formulierung ,,also das jetzt nochmal halb machen“ stützt jedoch die Annahme, dass sie sich auf die ikonische Bruchdarstellung im Kreis bezieht. Im Gegensatz zur Bearbeitung des ersten unvollständigen Beispiels scheint sie das Vergröbern der Einteilung in diesem Fall nicht als Zusammenfassen von Teilen zu verstehen, sondern nimmt an, dass sie die Teile des Ganzen „noch mal halb machen“ (3), bzw. noch einmal halbieren müsse. Es ist möglich, dass sie hierbei das Vorgehen mit dem Erweitern von Brüchen als Verfeinern einer Einteilung verwechselt und Handlungselemente dieses Verfahrens überträgt. Die beiden Schülerinnen beginnen zu zeichnen.

Ann-Katrin hält inne und stellt fest, dass der gekürzte Bruch „,3 Sechstel“ ist. Sie schaut auf das Arbeitsheft von Anita, beginnt die Teile in der Darstellung des Bruchs $\frac{6}{12}$ zu zählen und wirkt verwundert über die Anzahl der Teile in der ursprünglichen Einteilung. Ihre Verwunderung kann damit erklärt werden, dass sie entsprechend des Zählers des gekürzten Bruchs annimmt, in der ikonischen Darstellung drei Teile zu einem Teil zusammenfassen zu müssen (Perseverationsfehler), dann aber erkennt, dass dieses Vorgehen ausgehend von 12 Teilen nicht zu sechs gleichen Teilen führt. Sie sagt, dass sie ,,das gerade alles nicht“ (9) versteht und beginnt im Lösungsbeispiel am Bildschirm nach einer Kreisdarstellung zu suchen, die sie nicht findet und erneut bei ihrer Partnerin schaut. Da Ann-Katrin weiterhin Schwierigkeiten bei der Anwendung des Verfahrens zum Vergröbern auf die Kreisrepräsentation hat, kürzt sie den Bruch nun auf symbolischer Ebene und möchte den gekürzten Bruch nun zeichnerisch darstellen. Für die ikonische Darstellung versichert sie sich noch einmal bei Anita „wo da [in einem Bruch] der Nenner und wo [...] der Zähler [ist]“ (14-15). Anita antwortet ihr, dass der Zähler eines Bruchs oben und der Nenner eines Bruchs unten steht (16), worauf hin Ann-Katrin feststellt, dass sie den Kreis 
a)

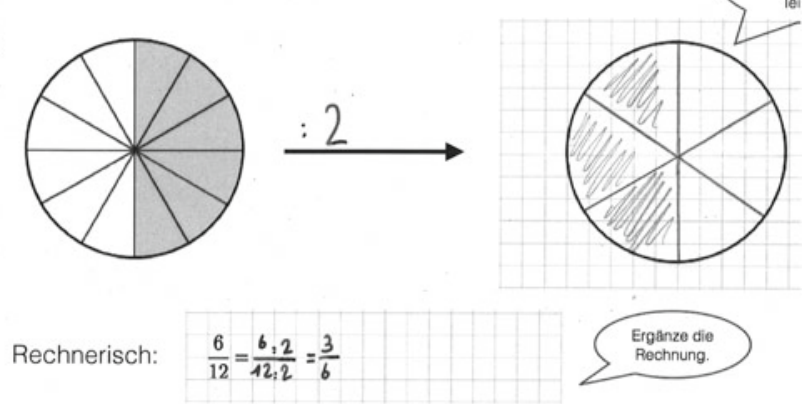

Abbildung 5.45 Ann-Katrins Lösung von Aufgabe 3.2 a)

„dann in 6 Teile teilen“ (17) müssen. Anita ergänzt, dass sie von den sechs Teilen „,3 davon“ (18) markieren müssen.

Nachdem Ann-Katrin noch einmal äußert, dass sie ,gar nichts mehr“ (19) verstehe und sagt, dass diese Aufgabe für sie die ,schwierigste“ sei und die anderen Aufgaben „besser“ (20-21) bzw. einfacher gewesen seien, beginnen die beiden Schülerinnen erneut zu zeichnen. Dabei stellt sich die Einteilung des Kreises in sechs gleich große Teile schwierig dar. Anita gelingt es letztendlich die neue Einteilung zu zeichnen und Anita und Ann-Katrin setzen ihre Bearbeitung in Einzelarbeit fort (Abb. 5.45).

Nach einiger Zeit des individuellen Arbeitens wendet sich Anita an ihre Partnerin und sagt, dass sie Aufgabenteil b) ,richtig“ habe und der gekürzte Bruch „,2 Viertel“" sei (30-31). Sie blickt auf das Arbeitsheft von Ann-Katrin und stellt fest, dass sie nicht nur bereits mit der Bearbeitung von Teilaufgabe b) fertig ist, sondern es zudem ,perfekter gemacht" habe als sie (31-32). In der Folge beschreibt Ann-Katrin ihr Vorgehen, dass die ,das vorher schon berechnet“ (33) habe bevor sie die neue Einteilung eingezeichnet habe. Ihre Erklärung deutet darauf hin, dass sie ihr Vorgehen angepasst hat und nicht mehr wie zu Beginn zunächst versucht hat auf ikonischer Ebene die neue Einteilung herzuleiten und getrennt davon den gekürzten Bruch zu berechnen, sondern nun zuerst auf symbolischer Ebene den Bruch gekürzt hat und diesen gekürzten Bruch schließlich zeichnerisch dargestellt hat. Während sie zuvor von der ikonischen Bruchdarstellung ausgegangen ist und auf ikonischer Ebene versucht hat, die Einteilung zu vergröbern, betrachtet sie nunmehr zunächst die symbolische Darstellung und zeichnet den gekürzten Bruch ein, ohne die Vergröberungshandlung nachzuvollziehen. 
Insgesamt können die Analysen der Bearbeitungsprozesse von Anita und AnnKatrin in den folgenden Deutungshypothesen zusammengefasst werden:

- Ann-Katrin und Anita übertragen sowohl das rechnerische Verfahren zum Kürzen von Brüchen sowie die Handlung des Vergröberns einer Einteilung auf ikonischer Ebene und wenden beide Verfahren auf einen neuen Bruch im ersten unvollständigen Beispiel an. Dabei interpretieren sie das Vergröbern einer Einteilung auf ikonischer Ebene als Zusammenfassen von Teilen zu einem größeren Teil.

- Der Repräsentationswechsel im zweiten unvollständigen Beispiel führt bei AnnKatrin dazu, dass sie die Verfahren nicht wie zuvor überträgt und anwendet. Ihre Schwierigkeiten bei der Übertragung äußern sich

- im Verwechseln des Kürzens von Brüchen als Vergröbern einer Einteilung mit dem Erweitern als Verfeinern einer Einteilung,

- in ihren Schwierigkeiten Teile in einer Kreisrepräsentation zusammenzufassen und allgemein einen Kreis in Sechstel einzuteilen, sowie

- in einem Perseverationsfehler, bei dem sie den Zähler des gekürzten Bruchs als Anzahl der Teile interpretiert, die zu einem Teil der Einteilung zusammen gefasst werden.

- Diese Schwierigkeiten führen im Fall von Ann-Katrin dazu, dass sie ihr Vorgehen ändert. Während sie zunächst stets von der ikonischen Bruchdarstellung ausgeht und die Einteilung vergröbert, indem sie die entsprechende Anzahl von Teilen zu einem Teil zusammenfasst, geht sie dazu über, zunächst den Bruch auf symbolischer Ebene rechnerisch zu kürzen und den gekürzten Bruch ikonisch darzustellen. Im Gegensatz zu ihrem Vorgehen im ersten unvollständigen Beispiel ist die Handlung des Vergröberns der Einteilung nicht mehr in ihrem Vorgehen zu erkennen, was als eine Trennung der Handlung auf ikonischer und symbolischer Ebene interpretiert werden kann. Es ist anzunehmen, dass die Schwierigkeiten, die sie bei der Anwendung des Verfahrens auf eine Bruchdarstellung in einem Kreis erfährt, dazu führt, dass sie ihren Fokus auf das rechnerische Vorgehen und die vertraute Darstellung von Brüchen in einer Kreisrepräsentation legt.

\section{Vergleich der Bearbeitungen der unvollständigen Beispiele}

Die Analysen der dargestellten Bearbeitungsprozesse zeigen, dass das Verfahren zum Kürzen von Brüchen von den Lernenden weitgehend fehlerfrei übertragen und angewendet wurde. Mit Bezug auf die Verknüpfung des rechnerischen Verfahrens mit der anschaulichen Vorstellung des Vergröberns einer Einteilung sind vor 
allem zwei Aspekte herauszustellen. In den Bearbeitungsprozessen ist eine Trennung der Verfahren auf ikonischer und symbolischer Ebene zu beobachten, die mit einer zunehmenden Fokussierung auf die Anwendung des rechnerischen Verfahrens einhergeht.

Trennung der Handlungen auf symbolischer und ikonischer Ebene: In den schriftlichen Produkten und den Bearbeitungsprozessen der Paare ist dokumentiert, dass die Schülerinnen und Schüler nach dem Lesen der animierten Lösungsbeispiele das rechnerische Kürzen von Brüchen mit dem Vergröbern einer Einteilung auf ikonischer Ebene in Verbindung gebracht haben.

Im Fall von Bennet und Julius konnte herausgearbeitet werden, dass obgleich zunächst eine Verbindung zwischen der rechnerischen Handlung und der anschaulichen Darstellung hergestellt wird, bereits in der Bearbeitung des ersten unvollständigen Beispiels voneinander getrennt werden. Dies äußert sich zum einen darin, dass ihre Lösungswege in zwei Teilen bestehen: Zuerst kürzen sie die Brüche auf symbolischer Ebene und stellen den gekürzten Bruch im zweiten Schritt anschaulich dar. Zum anderen sind Anzeichen dafür zu erkennen, dass sie die Bruchdarstellungen des ungekürzten und des gekürzten Bruchs als getrennte Entitäten betrachten. Sie erkennen zwar, dass in beiden Darstellungen der gleiche Anteil markiert ist, sie stellen dennoch keinen dynamischen Zusammenhang zwischen den beiden Darstellungen im Sinne einer Transformation der Einteilung her. Vor diesem Hintergrund interpretieren sie den gekürzten Bruch als ,neuen“ Bruch, wobei es möglich ist, dass diese Betrachtung durch die Formulierung im Lernmaterial unterstützt wird, in dem wiederholt gefordert wird, eine ,neue Einteilung“ einzuzeichnen, was als Darstellung eines „,neuen Bruchs“ interpretiert werden könnte.

Im Fall von Anita und Ann-Katrin weisen die Analysen darauf hin, dass insbesondere Ann-Katrin in der Bearbeitung des ersten unvollständigen Beispiels das Kürzen von Brüchen auf anschaulicher Ebene mit dem ,Zusammenfassen von Teilen“ interpretiert und entsprechend die Idee des Vergröberns einer Einteilung überträgt und anwendet. Der Repräsentationswechsel von einer Bruchdarstellung im Rechteck zu einer Bruchdarstellung in einer Kreisrepräsentation führt in ihrem Fall zu einer großen Irritation, die zur Folge hat, dass sie ihr Vorgehen im Vergleich zum ersten unvollständigen Beispiel ändert. Während sie in der Bearbeitung des ersten unvollständigen Beispiels vor allem die Handlung auf ikonischer Ebene fokussiert und zunächst die Einteilung vergröbert, indem sie die entsprechende Anzahl von Teilen in der Ausgangsdarstellung zusammenfasst, berechnet sie nach einigen fehlerhaften Ansätzen im zweiten unvollständigen Beispiel zunächst die gekürzten Brüche auf symbolischer Ebene und zeichnet im Anschluss die ikonischen Repräsentationen 
dieser Brüche. In ihrer Lösung und in ihrer Argumentation ist das Vergröbern der Einteilung als Zusammenfassen von Teilen nicht mehr enthalten.

Fokussierung auf die Anwendung des rechnerischen Verfahrens: Die beiden dargestellten Fälle eint, dass das rechnerische Verfahren zum Kürzen von Brüchen zunächst mit der Handlung des Vergröberns einer Einteilung auf anschaulicher Ebene verbunden wird, diese Verbindung jedoch in der Übertragung und Anwendung auf neue Anforderungssituationen zunehmend ausgeblendet wird und der Fokus auf die Anwendung des rechnerischen Verfahrens gelenkt wird. Während diese Fokussierung im Fall von Bennet und Julius bereits in der Bearbeitung des ersten unvollständigen Beispiels sichtbar wird, kann im Fall von Anita und Ann-Katrin die Entkopplung von symbolischer und anschaulicher Ebene auf den Repräsentationswechsel der Bruchdarstellungen in den Aufgaben zurückgeführt werden. Aufgrund von Schwierigkeiten in der Visualisierung von Anteilen in einer Kreisrepräsentation geht Ann-Katrin dazu über, nicht zunächst die Einteilung der ikonischen Bruchdarstellung zu vergröbern, sondern zuerst den Bruch auf symbolischer Ebene zu kürzen und dann den gekürzten Bruch einzuzeichnen.

Es ist anzunehmen, dass die Anforderungen der Übertragung und Anwendung der Verfahren in einer neuen Aufgabe dazu führen, dass die Schülerinnen und Schüler eher auf vertraute Handlungskonzepte zurückgreifen. In diesem Fall ist dies die Durchführung einer Division und die Darstellung von Brüchen in einer Kreis- oder Rechteckrepräsentation. Dabei tritt die Verknüpfung der Handlungen auf symbolischer und anschaulicher Ebene in den Hintergrund und führt möglicherweise dazu, dass diese Verknüpfung nicht weiter entwickelt und gestärkt wird, sodass es zu einer Entkopplung zwischen symbolischer und anschaulicher Ebene kommt. Diese Entwicklung könnte dazu führen, dass die Lernenden das Kürzen von Brüchen als Kalkül anwenden, ohne damit eine anschauliche Vorstellung zu verknüpfen.

Weitere Schwierigkeiten und fehlerhafte Übertragungen: Die dargestellten Analysen dokumentieren verschiedene Schwierigkeiten bei der Übertragung und Anwendung der Verfahren.

In beiden Bearbeitungen sind fehlerhafte Assoziationen im Sinne eines Perseverationsfehlers zu beobachten, bei denen einzelne Ziffern im Zähler nachwirken und zu falschen Lösungsansätzen führen. In diesem Zusammenhang interpretiert Julius die Formulierung ,eine Einteilung, die ein drittel so viele Teile hat" mit dem Einzeichnen des Bruchs $\frac{1}{3}$ und Ann-Katrin interpretiert den Zähler des gekürzten Bruchs als Anzahl der Teile, die zu einem Teil zusammengefasst werden. Beide Fälle deuten jedoch nicht auf ein robustes Fehlkonzept hin, sondern können als Anzeichen einer hohen kognitiven Belastung in der Transfersituation gedeutet werden. 
Besonders im Fall von Ann-Katrin ist zu beobachten, dass die Darstellung von Brüchen in einer Kreisrepräsentation zu Schwierigkeiten führt. Zunächst führt der Repräsentationstransfer von einer Darstellung der Brüche in einem Rechteck zu einer Bruchdarstellung im Kreis dazu, dass sie die Verfahren des Vergröberns und Verfeinerns einer Einteilung miteinander verwechselt und zum anderen fällt es ihr augenscheinlich schwer den gekürzten Bruch in einer Kreisrepräsentation einzuzeichnen und den Kreis in Sechstel einzuteilen. Es ist begründet anzunehmen, dass sie Probleme hat, sich Bruchteile in einer Kreisdarstellung vorzustellen, was eine Anpassung des Verfahrens an die veränderte Repräsentation zusätzlich erschwert.

\subsubsection{Vergröbern der Einteilung einer Strecke}

Unmittelbar auf die unvollständigen Beispiele folgt eine Aufgabe, die einen Repräsentationstransfer zur Darstellung von Brüchen an einer Strecke erfordert.

In der Aufgabe sind zwei identische Streckenabbildungen des Bruchs $\frac{8}{12}$ vorgegeben, deren Einteilung so verändert werden soll, dass die neue Einteilung halb so viele (Teilaufgabe a) und ein viertel so viele (Teilaufgabe b) hat als zuvor. Dazu sollen die Lernenden jeweils die neue Einteilung in die vorgegebenen Strecken einzeichnen und die dargestellten Brüche angeben. Zudem sollen die Lernenden angeben, wie man den Bruch in der neuen Einteilung rechnerisch erhält (vgl. Abb. 5.46).

\section{AufGabe 3}

Die Strecke ist in Zwölftel eingeteilt, davon sind 8 Zwölftel markiert. Die neue Einteilung soll a) halb so viele b) ein viertel so viele Teile haben wie vorher.

Zeichne die neue Einteilung in die Strecke und gib den Bruch in der neuen Einteilung an. Wie erhält man den Bruch rechnerisch?

a)

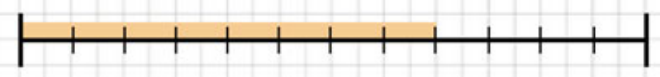

b)

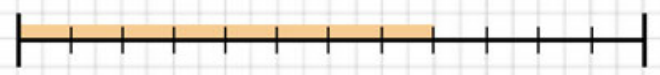

Abbildung 5.46 Aufgabe zum Vergröbern der Einteilung einer Strecke 
Für die Bearbeitung von Teilaufgabe a) wird somit erwartet, dass die Lernenden je zwei Teile der Strecke zu einem neuen Teil zusammenfassen, sodass die neue Einteilung der Strecke aus sechs Teilen besteht, von denen vier Teile markiert sind und entsprechend den Anteil $\frac{4}{6}$ darstellen. Rechnerisch entspricht diese Vergröberung der Einteilung der Division von Zähler und Nenner mit 2. Analog wird für die Bearbeitung von Teilaufgabe b) erwartet, dass die Lernenden eine neue Einteilung mit drei Teilen einzeichnen, wobei ein Teil der neuen Einteilung vier Teilen der ursprünglichen Einteilung entspricht. Die neue Einteilung der Strecke stellt somit den Bruch $\frac{2}{3}$ dar, den man rechnerisch durch die Division von Zähler und Nenner mit 4 erhält.

Transferprozesse: Der zentrale Transferprozess in dieser Aufgabe ist die Übertragung des Vergröberns einer Einteilung auf die Repräsentation von Brüchen an einer Strecke. Wohingegen im Lösungsbeispiel und in den unvollständigen Beispielen das Verfahren ausschließlich auf Rechteck- und Kreisrepräsentationen von Brüchen angewendet wurde, ist der Anteil am Ganzen einer Strecke eine Länge und kein Flächeninhalt. Das Verfahren zum Vergröbern einer Einteilung muss somit an die Eigenschaften einer Strecke angepasst werden.

Ein weiterer Transferprozess betrifft den in Aufgabenteil b) erstmals auftretenden Kürzungsfaktor 4. Im Lösungsbeispiel sowie in den unvollständigen Beispielen wurden ausschließlich die Kürzungsfaktoren 2, 3, und 6 thematisiert, womit das Vierteln der Teile der Anteile bzw. das Kürzen durch 4 eine neue Anforderung darstellt.

\section{Bennet \& Julius - Herleitung des Verfahrens zum Vergröbern einer Einteilung Die Bearbeitung beginnt mit einer hier nicht abgebildeten Diskussion über die Auf- gabenstellung. Nachdem Julius die Aufgabenstellung laut vorgelesen hat, disku- tieren die beiden Partner, ob sie eine neue Strecke zeichnen müssen, oder ob die vorgegebene Ausgangsdarstellung für die Bearbeitung genutzt werden kann. Sie rufen die Lehrkraft hinzu, die ihnen erklärt, dass sie sowohl eine neue Darstellung anfertigen, aber auch die Ausgangsdarstellung nutzen können. Nach dieser Klärung beginnen Bennet und Julius die eigentliche Bearbeitung.}

\section{Transkript B3A3 - Bennet \& Julius - Szene 1 - Aufgabe 3}

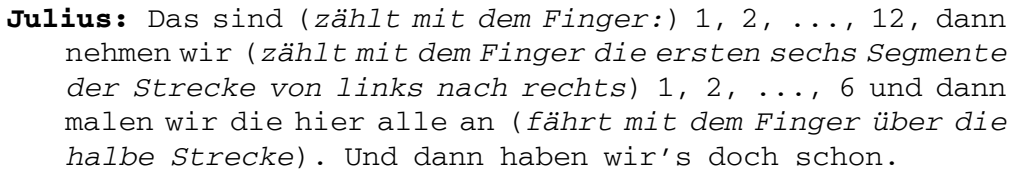




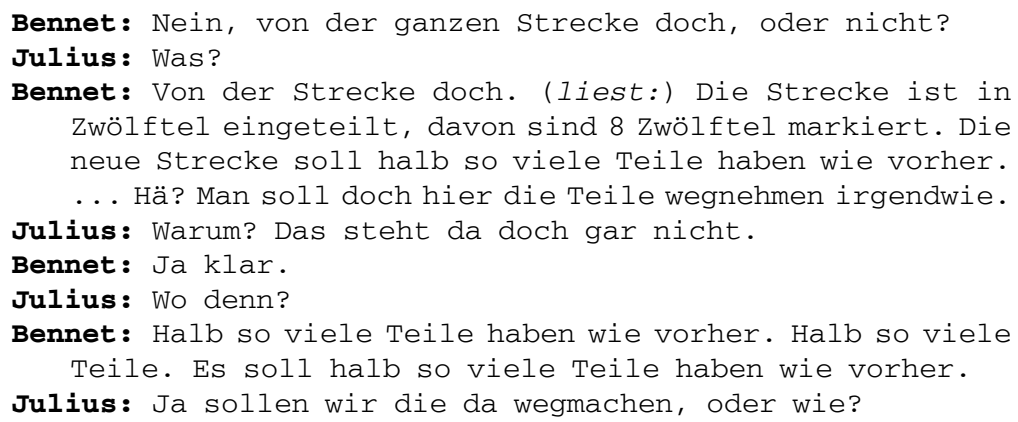

Obwohl der dargestellte Bruch angegeben ist, beginnt Julius die Bearbeitung damit die Anzahl der Teilsegmente der Strecke zu zählen. Er zählt zwölf Teile und schlägt vor, dass sie die ersten sechs Teile von links markieren (1-5), wobei er mit dem Finger die linke Hälfte der Strecke abfährt. Demnach interpretiert Julius das Einzeichnen einer neuen Einteilung mit halb so vielen Teilen als Markieren der halben Strecke ohne die Einteilung zu verändern.

Bennet widerspricht dem Vorschlag von Julius damit, dass $\frac{8}{12}$,,von der ganzen Strecke“ (6) markiert werden sollen. Was genau Bennet meint, lässt sich nicht direkt erschließen. Es ist jedoch anzunehmen, dass er entweder meint, dass die ganze Strecke neu eingeteilt werden soll, oder dass Julius den markierten Anteil ,von der ganzen Strecke“ verändert hat. Bennet liest die Aufgabenstellung noch einmal laut vor und ist irritiert von der Formulierung, dass die Strecke ,,halb so viele Teile wie vorher" haben soll, die er so versteht, dass man ,hier die Teile wegnehmen“ (8-11) soll. Er stellt scheinbar keinen Zusammenhang zum Lösungsbeispiel und den unvollständigen Beispielen her, in denen Aufgabenstellung in gleicher Weise formuliert ist.

Julius kann die Irritation von Bennet nur bedingt nachvollziehen und merkt an, dass in der Aufgabenstellung nicht steht, dass sie Teile wegnehmen sollen (12). Er nimmt dann jedoch den Einwand von Bennet auf und stellt selber in Frage, inwieweit sie Teile der Einteilung ,wegmachen“ (17) sollen.

\section{Transkript B3A3 - Bennet \& Julius - Szene 2 - Aufgabe 3}

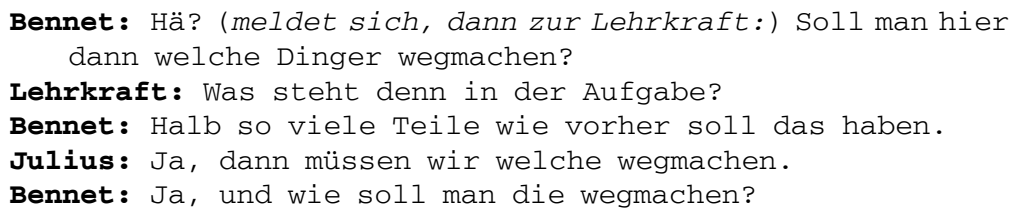


Lehrkraft: Sonst zeichnet ihr die Strecke noch mal mit derselben Länge da drunter.

Bennet: Ja, aber das geht doch gar nicht.

Julius: Doch, hier drunter, Bennet.

Bennet: Ja, aber dann muss ich ja wieder welche wegmachen.

Julius: Nein, du kannst einfach halb so viele malen. Ich mal da jetzt eine drunter.

Bennet: Aber es soll doch derselbe Bruch nachher sein.

Lehrkraft: Ja, kann es ja auch.

Julius: Das ist doch genau wie eben.

Lehrkraft: Das kriegt ihr zusammen schon hin. (geht weiter)

Bennet: Mach du das mal. (guckt bei Julius) Achso, wir sollen eine neue Strecke zeichnen?

Julius: Ja, ich mache das schon.

beide: (zeichnen individuell)

Bennet ruft die Lehrperson zu Hilfe und fragt, ob sie „Dinger“ bzw. Teile der Einteilung ,wegmachen“ (16-17) sollen. Die Lehrperson verweist auf die Aufgabenstellung und bittet sie diese noch einmal zu lesen (18). Auch nach wiederholtem lesen, erkennen Bennet und Julius keinen Zusammenhang zu den vorhergehenden Aufgaben und fragen erneut, wie sie die Teile der Einteilung ,wegmachen“ (20, 21) sollen, woraufhin die Lehrperson vorschlägt, dass sie ,die Strecke noch mal mit derselben Länge da drunter" (22-23) zeichnen. Bennet ist weiterhin irritiert und erwidert, dass das nicht gehe (24), da man so ,ja wieder welche wegmachen“ (26) solle.

Julius nimmt den Vorschlag der Lehrperson auf und bemerkt, dass er „einfach halb so viele [Teile] malen“ (27-28) könne, was Bennet damit kommentiert, dass es nachher ,doch derselbe Bruch“ (29) sein muss, womit er meint, dass der markierte Anteil der neuen Darstellung wertgleich zum Bruch der Ausgangsdarstellung sein muss. Es ist zudem möglich, dass Bennet annimmt, eine neue Einteilung mit halb so vielen Teilen einzeichnen zu müssen, die jedoch denselben Bruch, also wieder $\frac{8}{12}$, darstellt und er entsprechend nicht erkennt, dass auch eine Einteilung mit halb so vielen Teilen denselben Anteil repräsentieren kann.

Bennets Schwierigkeiten lassen sich auf die Bearbeitung der unvollständigen Beispiele zurückführen, in denen er jeweils zuerst die Brüche auf symbolischer Ebene gekürzt hat und in der Folge den gekürzten Bruch in die vorgegebene Repräsentationsvorlage eingezeichnet hat. Dabei hat er vermutlich keinen Zusammenhang zwischen den beiden Bruchdarstellungen hergestellt und das Vorgehen entsprechend auch nicht als Vergröbern der Einteilung wahrgenommen, sondern als „Kürzen“ und „Einzeichnen“. Da die vorliegende Aufgabenstellung jedoch explizit zum Verändern der Einteilung der Strecke auffordert, sieht er scheinbar keine Anwendungsbedingungen für sein vorhergehendes Vorgehen. 


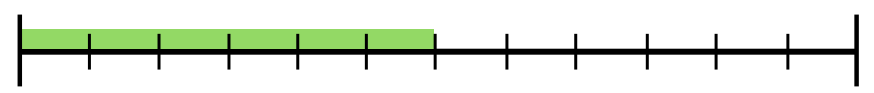

Abbildung 5.47 Rekonstruktion von Julius' Zeichnung in Teilaufgabe a)

Julius erkennt scheinbar den Zusammenhang zum Lösungsbeispiel und den unvollständigen Beispielen und erklärt, dass die Aufgabe ,doch genau wie eben“ (31) sei und beginnt eine neue Strecke zu zeichnen. In den Videoaufzeichnungen ist deutlich zu erkennen, dass er in seiner Zeichnung seinem anfänglichen Lösungsvorschlag folgt (siehe Abb. 5.47): Er zeichnet eine Strecke mit der Länge der vorgegebenen Strecke und übernimmt die Einteilung der Strecke in zwölf Teile, von denen er von links beginnend sechs Streckensegmente, also die halbe Strecke, färbt. Seine Zeichnung lässt darauf schließen, dass er das Einzeichnen einer neuen Einteilung mit halb so vielen Teilen mit dem Markieren der halben Strecke übersetzt. Auch er überträgt demnach nicht das Verfahren des Vergröberns einer Einteilung, da er die Einteilung unverändert lässt und einen veränderten Anteil des Ganzen markiert.

\section{Transkript B3A3 - Bennet \& Julius - Szene 3 - Aufgabe 3}

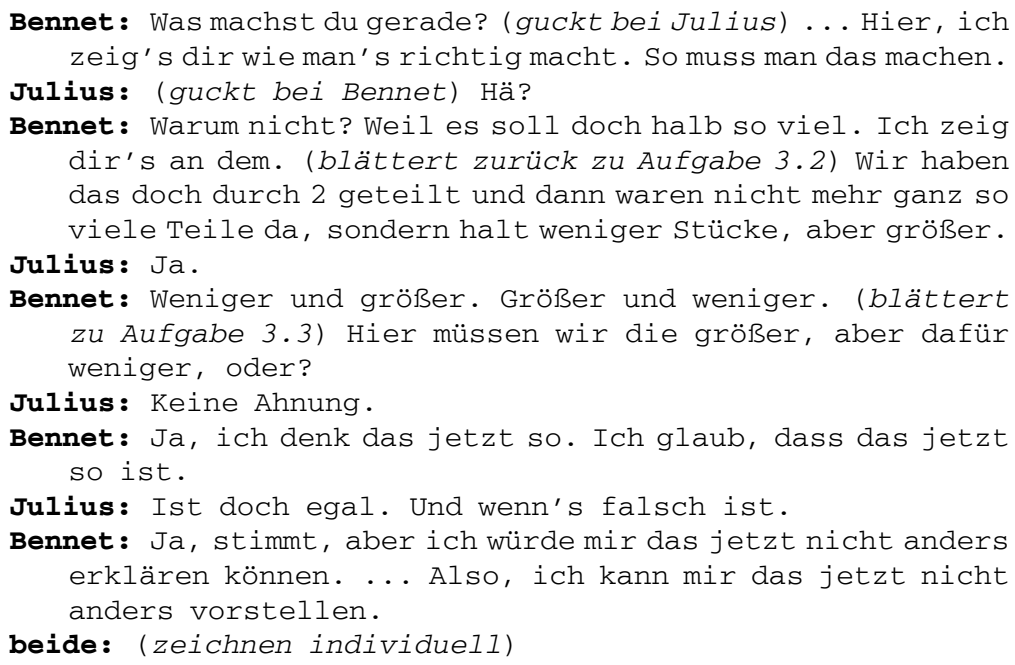
dir's an dem. (blättert zurück zu Aufgabe 3.2) Wir haben das doch durch 2 geteilt und dann waren nicht mehr ganz so viele Teile da, sondern halt weniger Stücke, aber größer. zu Aufgabe 3.3) Hier müssen wir die größer, aber dafür weniger, oder?

Julius: Keine Ahnung.

Bennet: Ja, ich denk das jetzt so. Ich glaub, dass das jetzt so ist.

Julius: Ist doch egal. Und wenn's falsch ist.

Bennet: Ja, stimmt, aber ich würde mir das jetzt nicht anders erklären können. ...Also, ich kann mir das jetzt nicht anders vorstellen.

beide: (zeichnen individuell)

Nachdem beide Schüler ein Zeit lang selbstständig gearbeitet haben, vergleicht Bennet seine Zeichnung mit der seines Partners. Er erkennt den Fehler von Julius und sagt, dass er ihm zeigen möchte, ,wie man's richtig macht“ (37-38). Julius schaut 
auf Bennets Zeichnung und scheint diese nicht zu verstehen (39). Zur Erklärung seiner Zeichnung blättert Bennet in seinem Arbeitsheft zurück zum zweiten unvollständigen Beispiel und erklärt, dass sie dort ,doch durch 2 geteilt“ haben und „dann [...] nicht mehr ganz so viele Teile da [waren], sondern halt weniger Stücke, aber größer[e]“" (40-43). Er überträgt diese Beobachtung auf die Bruchdarstellung in der vorliegenden Aufgabe und erklärt weiter: ,Weniger und größer. Größer und weniger. Hier müssen wir die größer, aber dafür weniger [machen]“ (45-47), anders könne er sich das nicht vorstellen (51-53).

\section{Transkript B3A3 - Bennet \& Julius - Szene 4 - Aufgabe 3}

Während Bennet bereits mit dem Zeichnen einer neuen Einteilung für Aufgabenteil b) beginnt, korrigiert Julius seine Lösung für Teilaufgabe a). Er radiert seine erste Zeichnung aus und zeichnet eine neue Strecke (Abb. 5.48). Nach dem Zeichnen einer neuen Strecke möchte er sich zunächst bei Bennet versichern, dass die Strecke in fünf Teile eingeteilt werden muss. Hierbei ist $\mathrm{zu}$ vermuten, dass er jedoch nicht meint, dass die Strecke in fünf Teile eingeteilt werden muss, sondern dass die Strecke mit fünf Markierungen (Teilstrichen) eingeteilt werden muss. Er schaut erneut bei Bennet und stellt fest, dass dieser, jetzt schon wieder die gleichen Stücke angemalt hat" obwohl die neue Einteilung ,halb so viele Teile haben“ (65-66) sollte wie zuvor. Er scheint folglich weiterhin davon überzeugt zu sein, dass nur die Hälfte des anfänglichen Anteils der Strecke markiert werden müsse und kann vermutlich nicht nachvollziehen, warum Bennet denselben Anteil wie zuvor markiert hat.

Bennet begründet seine Lösung schließlich noch einmal detailliert: „Gleich ist ja richtig, gleich so viel muss man machen. Nur, man muss das in andere Teile, in größere Stücke einteilen“ (67-69). Er erklärt somit, dass der markierte Anteil der Strecke beibehalten werden und lediglich die Größe der Stücke verändert wer- 


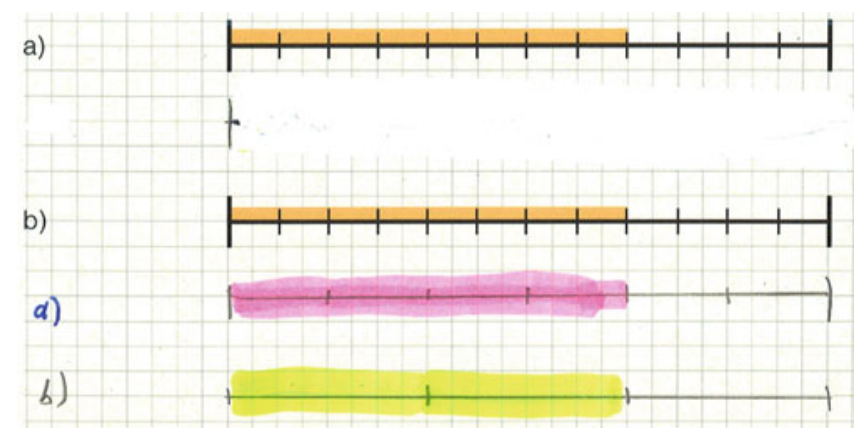

Abbildung 5.48 Julius Bearbeitung von Aufgabe 3.3

den muss. Julius kommentiert die Erklärung von Bennet nicht und übernimmt die Lösungen von seinem Partner.

In der Bearbeitung von Bennet und Julius fällt auf, dass sowohl der Ausgangsbruch sowie die gekürzten Brüche weder in der Kommunikation der beiden Schüler noch in den schriftlichen Dokumenten ihrer Bearbeitung auftauchen. Sie sprechen allein über die Anzahl und die Größe der Teile, stellen aber keinen erkennbaren Bezug zu den symbolischen Bruchrepräsentationen her.

Insgesamt wird in der Bearbeitung von Bennet und Julius deutlich, dass sie die unvollständigen Beispiele ohne die Anwendung der Handlung des Vergröberns der Einteilung gelöst haben. Da sie zuvor stets erst rechnerisch den gekürzten Bruch bestimmt haben, den sie danach ikonisch dargestellt haben, stellen sie keinen direkten Zusammenhang zwischen der ursprünglichen und vergröberten Einteilung her. $\mathrm{Da}$ in dieser Aufgabe jedoch vor allem das Vergröbern der Einteilung im Vordergrund steht, können sie ihr Vorgehen nicht direkt übertragen und erst durch die Reflexion der unvollständigen Beispiele scheint Bennet zu erkennen, was es bedeutet die Einteilung zu vergröbern.

Im Gegensatz zu seinem Partner äußert Julius gleich zu Beginn, dass sie lediglich die Hälfte der Strecke markieren müssen ohne die Einteilung zu verändern. Er verbindet demnach mit dem Vergröbern einer Einteilung keine Transformation bzw. Änderung der Einteilung des Ganzen, sondern eine Veränderung des Anteils im Sinne einer Verminderung.

Es ist zu anzumerken, dass beide Schüler in den fokussierenden Fragestellungen zum Lösungsbeispiel eingangs der Unterrichtsstunde die wesentlichen Aspekte des 
Vergröberns einer Einteilung auf ikonischer Ebene und dem rechnerischen Kürzen von Brüchen herausgestellt und notiert haben. Da sie die unvollständigen Beispiele auch ohne Anwendung der ikonischen Vergröberungshandlung lösen konnten, scheinen sie diese Beobachtungen in der Transfersituation nicht zu erinnern bzw. den entsprechenden Erfahrungsbereich nicht zu aktivieren. Dies führt im Fall von Bennet dazu, dass er zunächst nicht weiß, was er machen soll, und im Fall von Julius führt die ausbleibende Aktivierung dieses Erfahrungsbereichs zu einer individuellen und fehlerhaften Interpretation des Vorgehens.

Die nachfolgenden Beschreibungen und Argumentationen von Bennet deuten darauf hin, dass er das Verfahren zum Vergröbern einer Einteilung tatsächlich erst in dieser Aufgabe erstmalig anwendet und sich dieses - ungeachtet des Lesens des Lösungsbeispiels, der Beantwortung der fokussierenden Fragestellungen und der Bearbeitung der unvollständigen Beispiele - dazu zunächst neu herleiten muss, um es übertragen zu können.

Julius’ Äußerungen legen nahe, dass er das Konzept des Vergröberns einer Einteilung zur Herstellung von äquivalenten Bruchrepräsentationen nicht so erfasst hat, wie es aus didaktischer Sicht intendiert wurde. Die Tatsache, dass er seinen Partner darauf hinweist, dass das Vorgehen dasselbe sei wie in den unvollständigen Beispielen und seine Lösung einer fehlerhaften Konzeptualisierung des Verfahrens folgt, zeigt, dass es für die Bearbeitung der unvollständigen Beispiele nicht unbedingt erforderlich war, einen Zusammenhang zwischen der ursprünglichen Bruchrepräsentation und der Repräsentation des gekürzten Bruchs herzustellen. Stattdessen genügte es, das rechnerische Verfahren als Kalkül zu übertragen und anzuwenden und den gekürzten Bruch durch Anwenden des Verfahrens zur Darstellung von Bruchteilen einzuzeichnen.

Zuletzt ist in der Bearbeitung von Bennet und Julius zu erkennen, dass sie die markierten Anteile nicht aus den vergröberten Darstellungen ablesen und auch keine Beschreibung des entsprechenden rechnerischen Verfahrens angeben. Es ist anzunehmen, dass sie diesen Teil der Aufgabe schlichtweg übersehen. Andererseits kann diese Beobachtung als Anzeichen dafür gedeutet werden, dass Bennet und Julius weiterhin keine Verknüpfung zwischen den Handlungen auf anschaulicher und symbolischer Repräsentationsebene herstellen.

Insgesamt kann die Bearbeitung von Bennet und Julius in den folgenden Deutungshypothesen zusammengefasst werden:

- Das Verfahren zum Vergröbern einer Einteilung wird zunächst nicht übertragen und angewendet, da es zu Beginn der Bearbeitung noch nicht als solches erkannt und in Form eines Handlungsschemas konzeptualisiert wurde. Obwohl Bennet und Julius das Lösungsbeispiel eingehend lesen und alle wesentlichen Aspekte 
des Verfahrens in ihren Antworten auf die fokussierenden Fragestellungen herausstellen, konnte bereits in ihrer Bearbeitung der unvollständigen Beispiele eine Entkopplung des rechnerischen Verfahrens von der anschaulichen Handlung angenommen werden. Die Übertragung und Anwendung der anschaulichen Handlung war in ihren Lösungen nicht erforderlich, da sie die Brüche zunächst rechnerisch gekürzt haben und die gekürzten Brüche ohne einen direkten Bezug zur ursprünglichen Bruchdarstellung auf Grundlage des Bruchherstellungsverfahrens eingezeichnet haben. In der Folge ist anzunehmen, dass sie kein entsprechendes Handlungsschema zum Vergröbern einer Einteilung ausgebildet haben, das sie somit auch nicht auf die vorliegende Transferaufgabe übertragen können.

- Bennet leitet das Verfahren zum Vergröbern einer Einteilung in einem reflektierenden Rückgriff auf die vorhergehenden Lern- und Aufgabenmaterialien neu her. Dazu schaut er sich noch einmal genau an, was sie zuvor gemacht haben und es ist anzunehmen, dass er erst dadurch eine Beziehung zwischen den ursprünglichen Bruchdarstellungen und den Darstellungen der zugehörigen gekürzten Brüche herstellt und aus diesen die Vergröberungshandlung ableitet.

- Julius' Beschreibungen und Argumentationen dokumentieren ein Fehlkonzept, bei dem er das Vergröbern der Einteilung als Reduktion des Anteils interpretiert. Er deutet das Einzeichnen einer Einteilung mit halb so vielen Teilen als Reproduktion der ursprünglichen Strecke mit der ursprünglichen Einteilung und markiert dann jedoch unabhängig vom ursprünglichen Anteil die halbe Strecke. Dies deutet darauf hin, dass er das Konzept von äquivalenten Brüchen nicht aufgebaut bzw. erfasst hat und eine individuelle Deutung für die anschauliche Handlung des Vergröberns (einer Einteilung) entwickelt, die jedoch keine Veränderung der Einteilung des Ganzen beinhaltet. Auch diese Beobachtung kann als Anzeichen für Trennung der rechnerischen und anschaulichen Handlung interpretiert werden.

- Es wird ausschließlich die anschauliche Handlung des Vergröberns einer Einteilung durchgeführt und kein Bezug zur symbolischen Darstellungsebene durch Angabe der Anteile und Beschreibung des rechnerischen Vorgehens beim Kürzen von Brüchen hergestellt. Dies kann einerseits mit der hohen kognitiven Belastung durch die Transferanforderung begründet werden, stützt jedoch zusätzlich die Annahme einer getrennten Verarbeitung der rechnerischen und anschaulichen Handlung.

\section{Julia \& Marie - Veränderung des Ganzen}

Die Partnerarbeit von Julia und Marie ist dadurch charakterisiert, dass Marie im Wesentlichen allein die Aufgaben bearbeitet und Julia versucht die Lösungswege nachzuvollziehen. Dabei äußert Julia an mehreren Stellen Verständnisschwierigkei- 
ten, die Marie stets versucht mit ausführlichen Erklärungen aufzuklären. Julia kann den Erklärungen ihrer Partnerin in vielen Fällen jedoch nicht folgen und übernimmt in den meisten Fällen die Lösungen von ihrer Partnerin.

\section{Transkript B3A3 - Julia \& Marie - Szene 1 - Aufgabe 3}

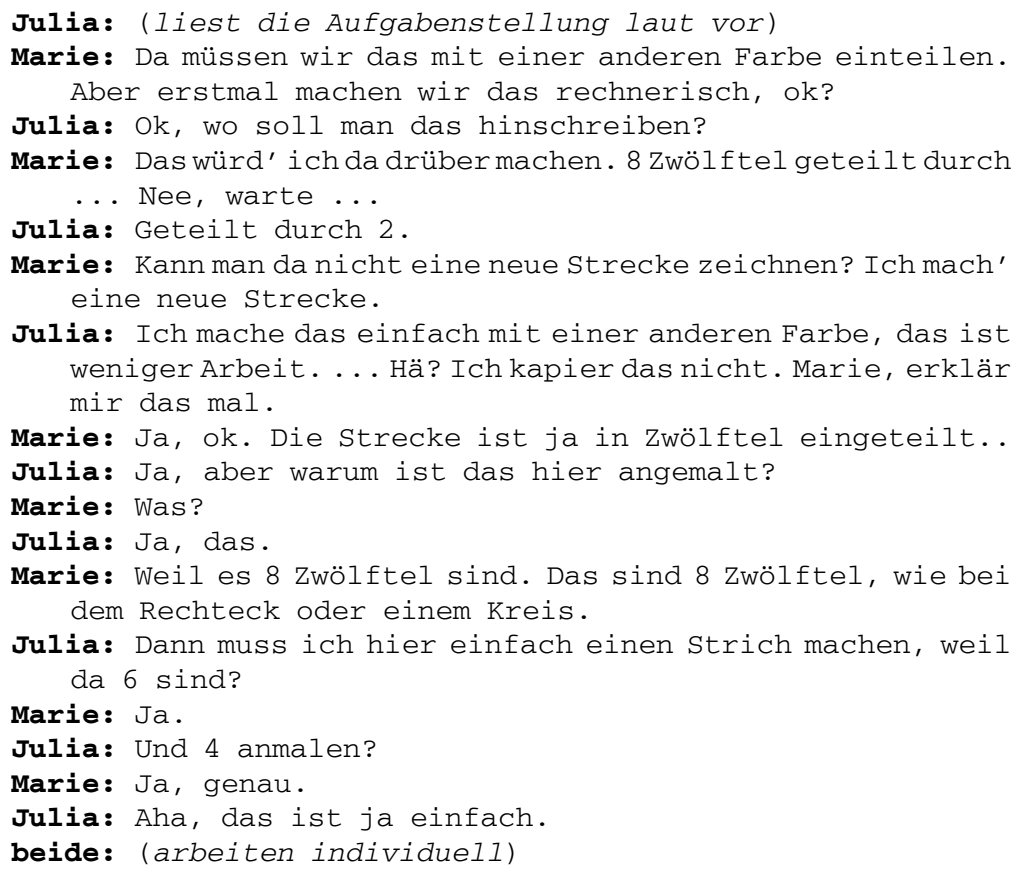

Unmittelbar nachdem Julia die Aufgabenstellung laut vorgelesen hat, sagt Marie, dass sie die Bruchdarstellung „mit einer anderen Farbe einteilen“ (2) sollen und schlägt vor zunächst die Brüche „rechnerisch“ (3) zu kürzen. Marie beginnt eine Rechnung zu formulieren und sagt ,8 Zwölftel geteilt durch ...“ (5-6), zögert dann jedoch und scheint einen Widerspruch zu entdecken: „Nee, warte ...“ (6). Julia nimmt die Rechnung ihrer Partnerin sofort auf und ergänzt, dass $\frac{8}{12}$,geteilt durch 2 “ (7) werden müssen. Die beiden Schülerinnen übertragen somit das Verfahren zum Kürzen von Brüchen und wenden es auf den vorgegebenen Bruch an.

Anstelle die Rechnung jedoch weiter auszuführen, nimmt Marie die zeichnerische Darstellung des neuen Bruchs in den Fokus: „Kann man da nicht eine neue Strecke zeichnen? Ich mach' eine neue Strecke“ (8-10). Julia ist mit diesem Vor- 
schlag nicht einverstanden und erklärt, dass sie ,das einfach mit einer anderen Farbe“ mache, da es so „weniger Arbeit“ (10-11) sei. Nach kurzem Überlegen stutzt sie jedoch und sagt, dass sie „das nicht [kapiert]“ (11) und bittet ihre Partnerin es ihr zu erklären.

In ihren Rückfragen zu der Erklärung von Marie wird deutlich, was genau Julia nicht versteht. Sie fragt, ,,warum [...] das hier [der Anteil $\frac{8}{12}$ ] angemalt“ (14) sei. Sie wundert sich, dass in der Strecke bereits ein Anteil farbig markiert ist, obwohl sie doch einen Anteil einzeichnen sollen. Julia interpretiert die Aufgabenstellung demnach nicht als Vergröbern der Einteilung des dargestellten Bruchteils, sondern als Einzeichnen eines neuen Bruchs, den sie zuvor berechnet haben. Ihre Nachfragen deuten darauf hin, dass sie das rechnerische Verfahren zum Kürzen mit keinen anschaulichen Vorstellungen verbindet und entsprechend auch keinen Zusammenhang zwischen der ursprünglichen Bruchdarstellung und der Darstellung des „,neu berechneten" Bruchs sieht.

Marie erklärt, dass es genauso sei, ,,wie bei dem Rechteck oder einem Kreis“ (1718), womit sie sich auf die Darstellungen in den unvollständigen Beispielen bezieht und deutlich macht, dass sie dasselbe Vorgehen auch hier anwenden müssen.

Aus dieser Erklärung schließt Julia, dass sie folglich ,hier einfach einen Strich machen“ muss, ,,weil da 6 sind“ (19-20). Damit meint sie, dass sie die Strecke halbieren, die Einteilung beibehalten und schließlich „4 [Teile] anmalen“ (22) möchte. Marie stimmt ihr zu und die beiden Schülerinnen setzen die Aufgabenbearbeitung individuell fort.

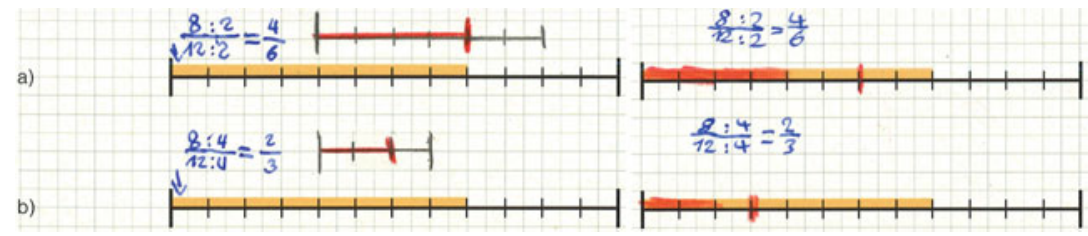

Abbildung 5.49 Maries (links) und Julias (rechts) Bearbeitung von Aufgabe 3.3

In den schriftlichen Dokumenten (vgl. Abb.5.49) von Marie und Julia ist zu erkennen, dass beide Schülerinnen keine Schwierigkeiten beim rechnerischen Kürzen des Bruchs durch die Faktoren 2 und 4 haben. Ebenso teilen sie ihre Ansichten darüber, wie die neue Einteilung einzuzeichnen sei, wobei sie diese jedoch unterschiedlich umsetzen.

Marie zeichnet für beide Teilaufgaben jeweils eine neue Strecke, für Teilaufgabe a) eine Strecke, die halb so lang wie die Ausgangsdarstellung ist, und für Aufga- 
benteil b) eine Strecke, die ein Viertel der Länge der Ausgangsdarstellung hat. Die Einteilung der Strecken verändert sie im Vergleich zur Ausgangsdarstellung nicht, und markiert somit in der ersten Strecke vier von sechs Teilen und in der zweiten Strecke zwei von drei Teilen.

Julia zeichnet dieselben Strecken in die Ausgangsdarstellung ein. Dazu trennt sie in Aufgabenteil a) die Hälfte der Strecke durch einen farbigen Strich ab und in Aufgabenteil b) ein Viertel der Strecke. Auch sie ändert die Einteilung der Strecke nicht und färbt in Teilaufgabe a) vier Streckensegmente und in Teilaufgabe b) zwei Streckensegmente farbig ein.

Obgleich die Bruchdarstellungen von Julia und Marie die korrekt gekürzten Anteile darstellen, zeichnen sie die Anteile ohne Bezug zur ursprünglichen Länge der Strecke neu ein, wobei sie die Länge der Strecke bzw. die Länge des Ganzen verändern. Die Größe der Teile der Einteilung verändern sie nicht, sondern übernehmen diese aus der ursprünglichen Darstellung (Abb. 5.49).

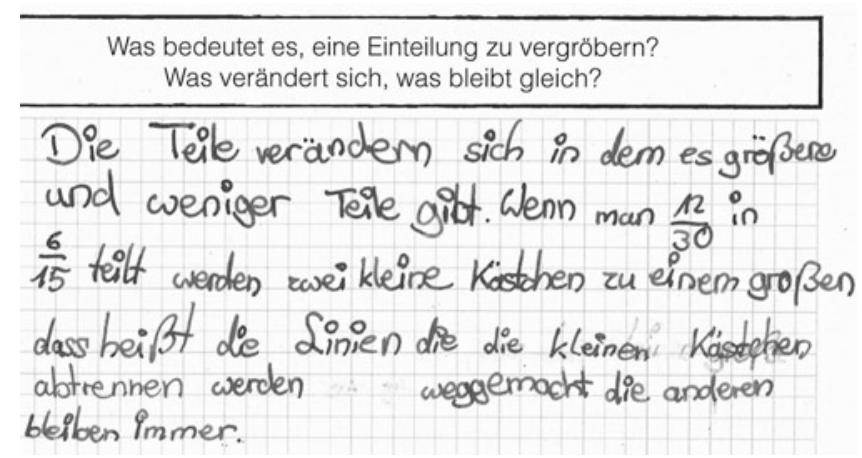

Abbildung 5.50 Maries Antwort auf die erste fokussierende Fragestellung zum Lösungsbeispiel

Vor dem Hintergrund, dass beide Schülerinnen in ihren Antworten auf die fokussierenden Fragen zum Lösungsbeispiel ausführlich das Verfahren zum Vergröbern einer Einteilung in eigenen Worten beschrieben haben (Abb. 5.50) und erklärt haben, dass ein Kürzen mit 2 auf anschaulicher Ebene dem Zusammenfassen von zwei Teilen zu einem großen entspricht, ist festzustellen, dass sie dieses Handlungsschema nicht auf die Darstellung an einer Strecke übertragen. Sie belassen die Teile der Einteilung so wie in der ursprünglichen Darstellung und halbieren bzw. vierteln dafür die Länge der ganzen Strecke. 
Insgesamt kann festgestellt werden, dass auch Julia und Marie keine Vorstellung vom Vergröbern einer Einteilung aktivieren und auf die Bruchdarstellung an einer Strecke anwenden. Stattdessen übertragen sie das rechnerische Verfahren zum Kürzen von Brüchen und stellen die berechneten neuen Bruchteile auf Grundlage des Bruchherstellungsverfahrens als neue Brüche dar. In ihren Lösungen ist kein Hinweis auf ein Verständnis dieses Vorgehens als Transformation der Einteilung zu erkennen.

Insgesamt kann die Bearbeitung von Julia und Marie in den folgenden Deutungshypothesen zusammengefasst werden:

- Das Verfahren zum Kürzen von Brüchen wird nicht mit der anschaulichen Handlung des Vergröberns einer Einteilung verbunden. Marie und Julia kürzen den vorgegebenen Bruch rechnerisch und stellen den gekürzten Bruch anschließend auf Grundlage des Bruchherstellungsverfahrens dar. Für ihre Darstellung des gekürzten Bruchs nutzen sie die gleiche Größe der Teile wie in der ursprünglichen Darstellung und verkürzen dafür die Länge der Strecke. Somit vergröbern sie nicht die Einteilung, sondern verändern die Größe des Ganzen und beachten dabei nicht die Äquivalenz der Bruchdarstellungen.

- Auch in der Bearbeitung von Julia und Marie ist eine Trennung des rechnerischen Verfahrens und der anschaulichen Handlung des Vergröberns zu erkennen. Anstatt die Einteilung der Bruchdarstellungen zu verändern, kürzen sie den vorgegebenen Bruch auf ikonischer Ebene und stellen diesen im Anschluss ikonisch dar.

\section{Glen \& Johanna - Vergröbern des markierten Anteils}

Die Partnerarbeit von Glen und Johanna ist sehr harmonisch und sie arbeiten sehr gewissenhaft und kooperativ. Sie haben das Lösungsbeispiel zu Beginn der Unterrichtsstunde ausführlich gelesen und auch grundlegende Eigenschaften des Verfahrens zum Kürzen von Brüchen und dem Vergröbern einer Einteilung in ihren Antworten auf die fokussierenden Fragestellungen zum Lösungsbeispiel notiert, wenn auch nicht sehr detailliert (vgl. Abb. 5.51). Sie beschreiben, dass beim Vergröbern, die Teile des Ganzen verändert werden, aber Wert des Bruches nicht verändert wird.

Glen und Johanna haben die unvollständigen Beispiele vollständig und korrekt gelöst, indem sie zunächst die Brüche entsprechend der Vorgaben rechnerisch gekürzt und die ikonischen Repräsentationen der gekürzten Brüche in den vorgegebenen Figuren eingezeichnet haben. In den Bearbeitungsprozessen der unvollständigen Beispiele ist zu erkennen, dass sie das rechnerische Verfahren zum Kürzen von Brüchen korrekt anwenden und die ikonischen Bruchrepräsentationen auf Grundlage des Bruchherstellungsverfahrens zeichnen. Eine Anwendung der Grundvorstel- 


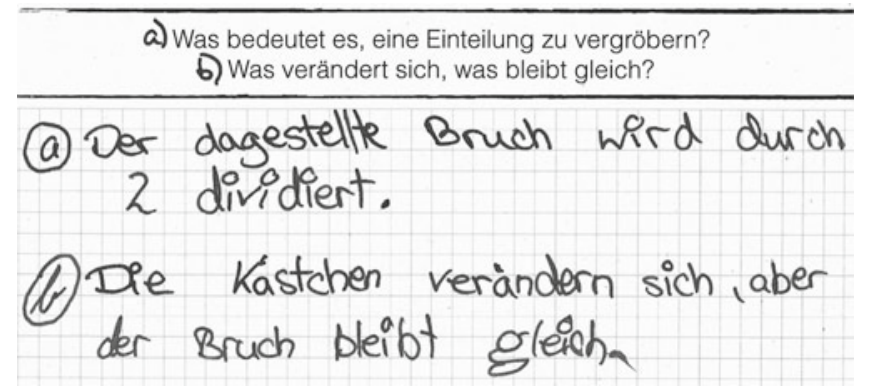

Abbildung 5.51 Johannas Antwort auf die erste fokussierende Fragestellung zum Lösungsbeispiel

lung vom Vergröbern einer Einteilung ist in der Interaktion von Glen und Johanna nicht festzustellen. Das nachfolgende Transkript dokumentiert ihre Bearbeitung zum Vergröbern der Einteilung einer Strecke.

\section{Transkript B3A3 - Glen \& Johanna - Szene 1 - Aufgabe 3 \\ Johanna: (liest die Aufgabenstellung laut vor) \\ Glen: Ich weiß es. \\ Johanna: Das erste ist ja leicht. Das sind ja halb so viele. Da muss man die Hälfte rechnen. \\ Glen: Hä? Wie? Die Hälfte rechnen? \\ Johanna: Die Strecke ist in Zwölftel eingeteilt. Davon sind 8 Zwölftel markiert und davon soll man halb so viele haben. Verstanden? So, das sind 8 geteilt durch 2 sind 4 . Du hast das bei Sechsteln gemacht.}

Glen: Oh, sorry.

beide: (arbeiten individuell)

Johanna: So, jetztschreibichdahinter... Das sind 4 Zwölftel.

Glen: Nein, 4 Sechstel.

Johanna: Wie kommst du auf 4 Sechstel?

Glen: So müsste das eigentlich sein. Du musst ja eigentlich die Hälfte von allem nehmen. 12 geteilt durch 2 gleich 6 .

Johanna: Hä? Und wie kommst du auf 12 geteilt durch 6? $\ddot{\text { Äh, }}$ geteilt durch 2 ?

Glen: Halb so viele. ... Achso, vom orangenen nur? Ach ja ... Ein viertel so viele.

Johanna: Einviertel soviele, ähm ...8 geteilt durch 4 sind 2 .

Glen: Achso ... Ach, das ging ja einfach.

Johanna: Sind 2 Zwölftel. 
Unmittelbar nach dem Lesen der Aufgabenstellung äußern Glen und Johanna, dass sie wissen, was zu tun ist und das die erste (Teil-) Aufgabe ,ja leicht“ sei (2, 34). Johanna erklärt, dass „man die Hälfte rechnen“ müsse, da es ,,ja halb so viele“ sein sollen (3-4). Sie erklärt weiter, dass die Strecke in "Zwölftel eingeteilt“ sei und acht Zwölftel markiert seien, von denen sie ,halb so viele“ haben wollen (68). Aus diesem Grund müssen sie „8 geteilt durch 2 sind 4“ rechnen müssen (8). Zudem stellt Johanna einen Bezug zu den Lösungsbeispielen her, indem sie sagt, dass Glen dasselbe im zweiten unvollständigen Beispiel gemacht habe (9). Obwohl Glen Johannas Vorschlag zunächst nicht einordnen konnte oder womöglich nicht verstanden hat, was sie mit ,die Hälfte rechnen“ (5) meint, scheint er ihrer Erklärung zuzustimmen und die beiden beginnen jeder für sich zu zeichnen.

In dieser Szene ist zu erkennen, dass Johanna das rechnerische Verfahren zum Kürzen von Brüchen überträgt und auf den vorgegebenen Bruch anwendet. Dazu bezieht sie sich allein auf den Aufgabentext und stellt zunächst keine Beziehung zu der vorgegebenen Streckendarstellung her. Ihr Fokus liegt allein auf dem Kürzen des Bruchs und es ist anzunehmen, dass sie dasselbe Vorgehen wie in ihren Lösungen der unvollständigen Beispiele anwendet, in denen Glen und Johanna auch zunächst auf symbolischer Ebene den neuen Bruch berechnet haben und diesen dann eingezeichnet haben.

Für Glens anfängliche Irritation können zwei Ursachen angenommen werden. Zum einen ist es möglich, dass er Johannas Formulierung „Das sind ja halb so viele. Da muss man die Hälfte rechnen“ nicht versteht und entsprechend nachfragt, was sie damit meint. Es ist jedoch auch möglich, dass er anders als Johanna, die ihren Lösungsansatz zunächst allein auf dem Aufgabentext aufbaut, seinen Fokus auf die Bruchdarstellung in der Strecke richtet und Johannas Lösungsvorschlag aus der ikonischen Perspektive nicht nachvollziehen kann. Da er nach Johannas ausführlicheren Erklärung jedoch keine weiteren Nachfragen stellt und umgehend zum selbstständigen Zeichnen und Schreiben übergeht, kann seine Reaktion „oh sorry“ (10) so gedeutet werden, dass seine Missverständnisse bezüglich Johannas Lösungsvorschlag aufgeklärt worden sind und dieses Vorgehen auch seinem ungeäußerten Lösungsansatz (2) entspricht.

In ihrer Zeichnung markiert Johanna (vgl. Abb. 5.52) den Anfangs- und den Endpunkt des markierten Streckenanteils mit einer längeren Teilmarkierung und markiert zudem die Mitte des markierten Anteils mit einem weiteren kleineren Teilstrich. Sie kommentiert ihre Zeichnung mit „das sind 4 Zwölftel“ (12-13). Es wird deutlich, dass sie nicht die ganze Strecke neu einteilt, sondern den markierten Anteil als Ganzes deutet und diesen in zwei gleich große Teile teilt. Sie interpretiert somit das Einzeichnen einer neuen Einteilung mit halb so vielen Teilen als Halbieren des Anteils, ohne die Einteilung des Ganzen zu verändern. Das rechnerische Verfahren 
a)

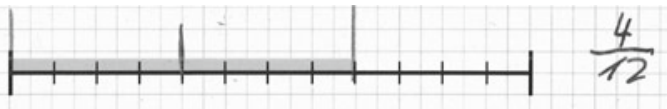

b)

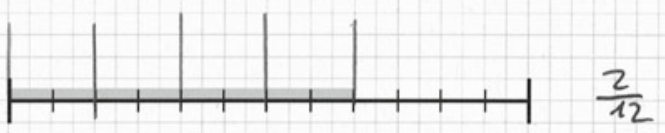

Abbildung 5.52 Johannas Bearbeitung von Aufgabe 3.3

zum Kürzen von Brüchen wendet sie zudem nur auf den Zähler des vorgegebenen Bruchs an und teilt diesen durch 2. Dass sie damit den Wert des Bruchs und somit den Anteil am Ganzen verändert, scheint sie nicht wahrzunehmen. Es ist möglich, dass es sich bei dieser Lösung um eine Übergeneralisierung der Aufgabenstellung handelt, die sie zunächst rezitiert: „Die Strecke ist in Zwölftel eingeteilt. Davon sind 8 Zwölftel markiert und davon [Hervorhebung des Autors] soll man halb so viele haben“ (6-8). Es ist somit denkbar, dass sie die Aufgabenstellung nicht auf die ganze Darstellung, sondern ausschließlich auf den markierten Teil der Strecke bezieht. Aber auch in diesem Falle zeigt ihre Lösung auch keine Veränderung der Einteilung, sondern ein Halbieren des markierten Teils der Strecke. Es kann somit angenommen werden, dass sie das Handlungskonzept des Vergröberns einer Einteilung nicht überträgt, sondern ein individuelles Erklärungsmodell entwickelt, dass aus ihrer Sicht zur Aufgabenstellung passt.

Im Gegensatz zu Johanna überträgt Glen das rechnerische Verfahren zum Kürzen von Brüchen korrekt auf den Bruch $\frac{8}{12}$, denn er widerspricht Johannas Lösung und erklärt, dass der neue Bruch „4 Sechstel“ (14) sein müsste. Er erklärt: „Du musst ja eigentlich die Hälfte von allem nehmen. 12 geteilt durch 2 gleich 6“ (15-16).

Johannas Missverständnis zeigt sich in ihrer Nachfrage, wie Glen auf die Rechnung ,12 geteilt durch [...] 2“ komme (17-18). Dies ist insbesondere vor dem Hintergrund ihrer korrekten Anwendung des Kürzens von Brüchen in den unvollständigen Beispielen bemerkenswert und ihren Partner auch zuvor auf das dort angewendete Vorgehen erinnert (9). Diese Divergenz ihres Vorgehens kann über die geänderten Handlungsmöglichkeiten und -Einschränkungen sowie den Repräsentationstransfer in dieser Aufgabe erklärt werden.

Im Gegensatz zu der Darstellung von Brüchen in einem Kreis oder Rechteck wird das Ganze durch die Fläche der zweidimensionalen Figur repräsentiert, was eine gesonderte Betrachtung eines Anteils als Ganzes sehr komplex gestaltet, da der markierte Anteil aus der Figur herausgelöst werden müsste und insbesondere ausgehend von einer Kreisrepräsentation eine abstrakte Fläche ergeben würde. Die 
Strecke als Ganzes wird hingegen nur eindimensional durch ihre Länge definiert. Das Loslösen des markierten Anteils von der ganzen Strecke ist somit allein durch ein Abtrennen des nicht markierten Streckenteils möglich.

Zudem unterscheidet sich die äußere Gestaltung der Streckenaufgabe deutlich von der Gestaltung der unvollständigen Beispiele. Während in den unvollständigen Beispielen die Rechnung zum Kürzen der Brüche notiert werden sollte und zum Einzeichnen der Bruchteile eine freie Repräsentation vorgegeben war, ist dies in dieser Aufgabe nicht der Fall. Es ist keine freie Repräsentation vorgegeben und in der äußeren Gestaltung der Aufgabe ist keine Fläche zum Notieren der Rechnung vorgesehen. Die veränderte äußere Gestaltung der Aufgabe trägt somit zur Vergrößerung der Transferdistanz zu den unvollständigen Beispielen bei und kann als eine Ursache für Johannas Änderung des Vorgehens verstanden werden. Johannas Abweichung vom Vorgehen in den Lösungsbeispielen deutet zudem darauf hin, dass sie über die Bearbeitung von diesen kein stabiles Handlungsschema ausgebildet hat, dass sie auf die neue Anforderung übertragen kann. Stattdessen entwickelt sie in der neuen Anforderung ein neues Vorgehen.

Glen erkennt, dass Johanna sich allein auf den markierten Anteil in der Streckendarstellung bezieht und hält ihre Lösung für plausibel. Er bemerkt ,achso, vom orangenen nur" (19) und übernimmt den Lösungsweg seiner Partnerin, sodass sich die beiden darüber einig werden, dass der neue Anteil in Teilaufgabe b) „2 Zwölftel“ heißen muss, und sie entsprechend ihres Vorgehens in Teilaufgabe a) den markierten Teil der Strecke in vier gleiche Teile einteilen, von denen sie einen Teil als den neuen Bruch interpretieren.

Die Bearbeitung von Glen und Johanna kann somit in den folgenden Deutungshypothesen zusammengefasst werden:

- Das Vorgehen aus der Bearbeitung der unvollständigen Beispiele wird nicht auf die neue Anforderung übertragen. Glen und Johannas Lösungen der unvollständigen Beispiele dokumentieren, dass sie das Verfahren zum Kürzen als Division von Zähler und Nenner durch denselben Divisor korrekt auf neue Brüche angewendet, und die gekürzten Bruchteile auch korrekt ikonisch dargestellt haben. In dieser Aufgabe ändern sie jedoch ihr Vorgehen in folgenden Aspekten:

- Sie lösen den markierten Anteil von der ganzen Strecke und interpretieren diesen als neues Ganzes,

- sie teilen ausschließlich den markierten Anteil der Strecke neu in zwei und in vier gleiche Teile ein und belassen den nicht markierten Anteil unverändert, und 
- sie teilen nur den Zähler des Bruchs und behalten den Nenner des ursprünglichen Bruchs bei.

Obwohl Glens Äußerungen zeigen, dass er das rechnerische Verfahren zum Kürzen zunächst korrekt anwendet, übernimmt er Johannas Änderung des Vorgehens. Ihre Anwendung der Division allein auf den Zähler des Ausgangsbruchs kann als Anpassung des rechnerischen Verfahrens an ihre anschauliche Handlung verstanden werden, da sie lediglich den markierten Anteil als Repräsentation des Zählers neu einteilt. Das Beibehalten des ursprünglichen Nenners kann in diesem Zusammenhang mit dem Beibehalten der ursprünglichen Einteilung der ganzen Strecke in zwölf Teile interpretiert werden.

\section{Can \& Philip - Übertragen und Beibehalten von Fehlkonzepten}

Can und Philip arbeiten weiterhin sehr unkonzentriert und lesen das Lösungsbeispiel nur flüchtig. Bereits in ihren Antworten auf die fokussierenden Fragestellungen ist zu erkennen, dass sie sowohl das rechnerische Verfahren zum Kürzen von Brüchen, wie auch das anschauliche Vergröbern der Einteilung nicht erfassen. So formuliert Philip als Antwort auf die dritte fokussierende Fragestellung, was das Teilen von Zähler und Nenner durch 6 auf anschaulicher Ebene in der Rechteckdarstellung des Bruchs $\frac{12}{30}$ ändert, dass der Anteil gleich bleibt und nur die Stücke kleiner werden. Als Veranschaulichung zeichnet er ein Rechteck, dass er in 30 Teile unterteilt, von denen er fünf Teile farbig markiert. Seine Zeichnung folgt dem folgenden Denkmuster: Er wendet die Division durch 6 nur auf den Nenner des Bruchs und nicht auf den Zähler an und rechnet $30: 6=5$. Das Ergebnis dieser Rechnung interpretiert er als Zähler des gekürzten Bruchs, für dessen Nenner der Nenner des Ausgangsbruchs übernommen wird. Er kürzt den Bruch $\frac{12}{30}$ demnach zu $\frac{5}{30}$, den er ikonisch neben seiner Antwort darstellt (siehe Abb. 5.53).

In den Bearbeitungen der unvollständigen Beispielen wird dieses fehlerhafte Vorgehen abgewandelt. So lautet seine Rechnung zum Kürzen des Bruchs $\frac{6}{18}$ mit dem Faktor 6: „, $\frac{6}{18}: \frac{1}{6}=3$ “ (siehe Abb. 5.54). Wie in seiner Antwort auf die fokussierende Fragestellung teilt er lediglich den Nenner des Bruchs durch den Kürzungsfaktor und interpretiert diesmal jedoch das Ergebnis dieser Division als Ergebnis der Kürzung bzw. als natürliche Zahl 3. Dieses Ergebnis interpretiert er auf anschaulicher Ebene als Anzahl der zu markierenden Teile in einer unveränderten Ausgangsrepräsentation.

In der Bearbeitung des zweiten unvollständigen Beispiels wird das Verfahren erneut angepasst. Philip „,kürzt“ den Bruch $\frac{6}{12}$ wie zuvor, indem er lediglich den Nenner des Bruchs durch 6 dividiert und das Ergebnis dieser Division als Ergebnis interpretiert. Auf anschaulicher Ebene übernimmt er nunmehr jedoch nicht 
Zähler und Nenner werden durch 6 geteilt.

Was verändert sich im Rechteck, was bleibt gleich?

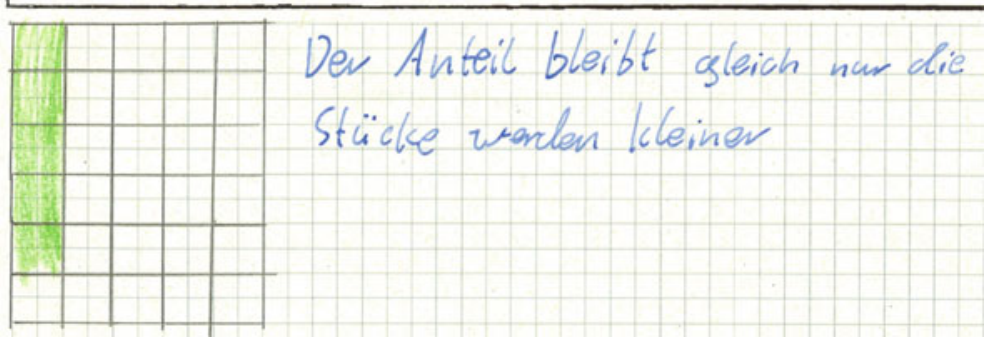

Abbildung 5.53 Philips Antwort auf die dritte fokussierende Frage zum Lösungsbeispiel
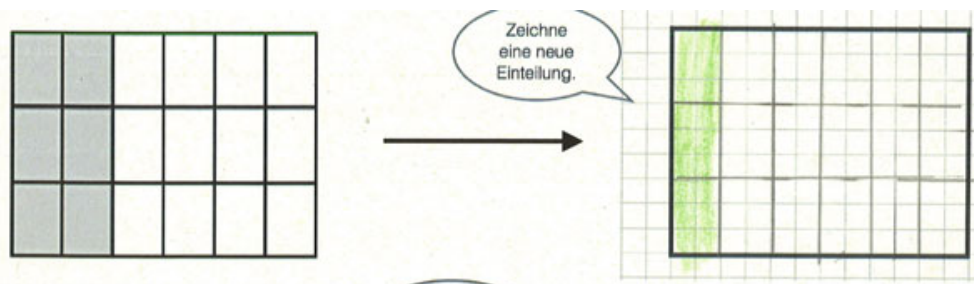

Rechnerisch:

Ergånze.

Man dividiert... Zähler uncl lénner dunch6 $\frac{6}{18}=: \frac{1}{6}=3$

Man nennt dies:

Kürzen durch ... 6

Abbildung 5.54 Philips Lösung von Aufgabenteil b) des ersten unvollständigen Beispiels

die Einteilung der ursprünglichen Bruchdarstellung, sondern deutet das Ergebnis der Division als Anzahl der Teile der neuen Einteilung. Einen Anteil markiert er nicht, sondern betrachtet das Vergröbern mit diesem Schritt als abgeschlossen (siehe Abb. 5.55). Sein Partner Can übernimmt alle Lösungen seines Partners. Das folgende Transkript dokumentiert ihre Übertragung dieses Vorgehens auf das Vergröbern von Anteilen in einer Streckendarstellung. 

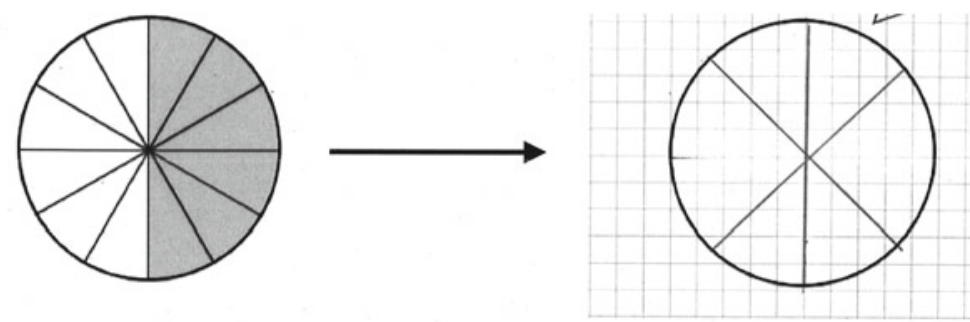

Rechnerisch:

$$
\frac{6}{12}=: \frac{1}{2}=6
$$

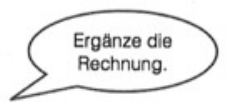

Abbildung 5.55 Philips Lösung von Aufgabenteil a) des zweiten unvollständigen Beispiels

\section{Transkript B3A3 - Can \& Philip - Szene 1 - Aufgabe 3 a)}

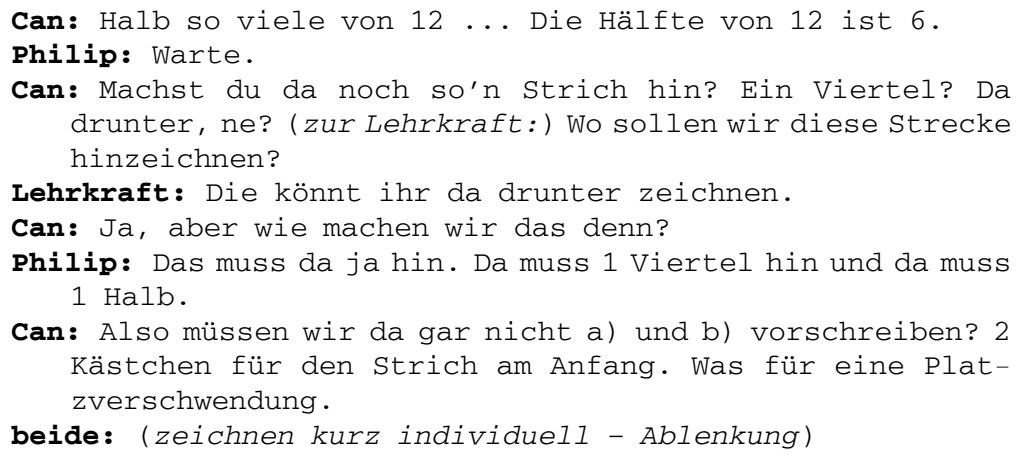

Die Bearbeitung von Can und Philip beginnt mit einer Klärung der Aufgabenstellung. Can berechnet „die Hälfte von 12“ (1), weiß jedoch nicht, wo er „,diese Strecke hinzeichnen“ (4-5) soll und wendet sich mit dieser Frage an die Lehrkraft, die ihnen rät unter der Ausgangsdarstellung eine neue Strecke zu zeichnen. Can erklärt jedoch, dass er nicht wisse, ,wie“ (7) er das tun soll. Philip kommentiert, dass sie unter die Darstellung von a) „1 Halb“ und unter die Darstellung von b) „1 Viertel“ (8-9) zeichnen müssen, bevor die beiden Partner zunächst individuell zu zeichnen beginnen und sich schließlich ablenken (13).

\section{Transkript B3A3 - Can \& Philip - Szene 2 - Aufgabe 3 a)}

Can: Ich mach jetzt einfach wieder diese ganze strecke mit gleich so vielen Strichen, oder? 

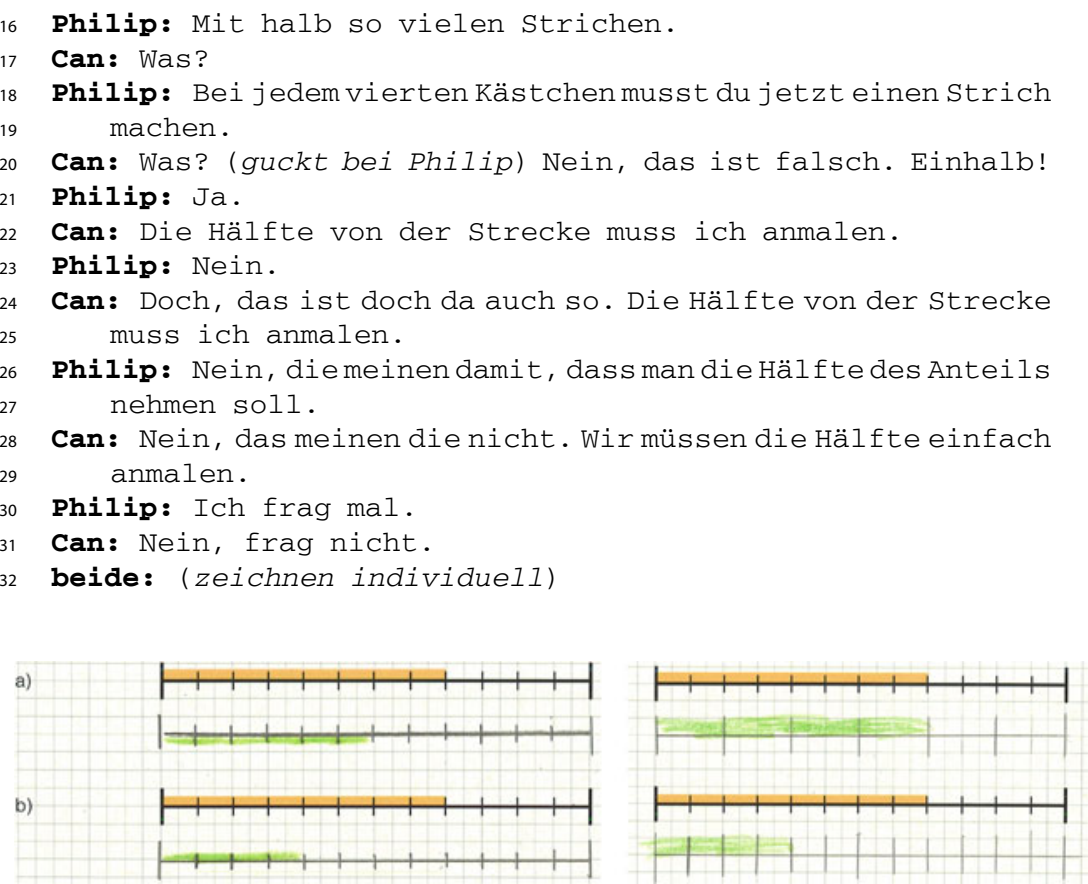

Abbildung 5.56 Can (links) und Philips (rechts) Bearbeitung von Aufgabe 3.3

Als sich Can und Philip wieder der Bearbeitung zuwenden erklärt Can, dass er ,jetzt einfach wieder diese ganze Strecke mit gleich so vielen Strichen“ (14-15) zeichnet. Philip widerspricht diesem Vorschlag und merkt an, dass sie eine neue Strecke ,mit halb so vielen Strichen“ (16) zeichnen sollen, indem sie ,bei jedem vierten Kästchen [...] einen Strich machen“ (18-19). Dieser Beschreibung nach fasst Philip je zwei Teile der Ausgangsstrecke zu einem neuen Teil zusammen und zeichnet eine neue Einteilung der Strecke in sechs Teile (vgl. Abb. 5.56), von denen er vier Teile färbt.

Can ist mit diesem Vorgehen jedoch nicht einverstanden und erklärt Philips Zeichnung für falsch (20). Stattdessen müsse ,,die Hälfte von der Strecke“ (22; 24-25; 28-29) angemalt werden, weil ,das [...] doch da [in den unvollständigen Beispielen] auch so“ (24) sei. Obgleich er einen Bezug auf ihre Lösungen der unvollständigen Beispiele herstellt, folgen seine Erklärungen einem anderen Denkmuster. Im Gegensatz zu ihren Deutungen im Lösungsbeispiel interpretiert er das Vergröbern der Einteilung der Strecke, sodass die neue Einteilung halb so viele Teile hat wie zuvor, als Markieren der Hälfte der Strecke. Er überträgt ihr Vorgehen aus 
den unvollständigen Beispielen demnach nicht, sondern interpretiert das Verfahren erneut auf eine andere Weise. Es ist jedoch anzunehmen, dass die Lösungen der unvollständigen Beispiele lediglich bei Philip abgeschrieben hat, ohne die dahinterliegende(n) Argumentation(en) nachzuvollziehen. Vor diesem Hintergrund kann angenommen werden, dass diese Sichtweise seinem individuellen Verständnis des Verfahrens entspricht, die er an dieser Stelle zum ersten mal äußert.

Anstatt sein Vorgehen aus den vorhergehenden Bearbeitungen zu übertragen deutet auch Philip die Aufgabenstellung bzw. die Handlungsanweisung eine neue Einteilung für die Strecke mit halb so vielen Teilen zu zeichnen neu. Gemäß der Aufgabenstellung fasst er je zwei Teile der ursprünglichen Darstellung zu einem Teil zusammen und zeichnet eine neue Strecke mit der korrekten Einteilung in nunmehr sechs Teile. Er markiert vier dieser Teile und somit den äquivalenten Anteil zur ursprünglichen Darstellung und begründet dies damit, dass ,man die Hälfte des Anteils nehmen soll“ (26-27), was jedoch seiner Zeichnung widerspricht, da er denselben Anteil der Strecke farbig markiert.

\section{Transkript B3A3 - Can \& Philip - Szene 3 - Aufgabe 3 b)}

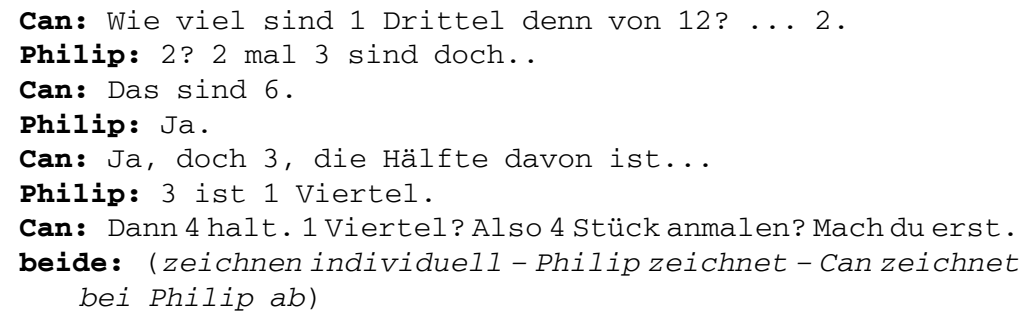

In ihrer Lösung des zweiten Aufgabenteils, in dem die neue Einteilung nur noch ein viertel so viele Teile wie zuvor haben soll, ändert Philip erneut sein Vorgehen und orientiert sich nun an der Argumentation von Can, der zunächst ohne erkennbaren Grund „1 Drittel von 12“ berechnen will (34). Can kommt für diese Rechnung zu dem Ergebnis „„2“ (34). Philip scheint Cans Rechnung nicht nachvollziehen zu können und erwidert, dass ,2 mal 3“ 6 seien (35), woraufhin Can ergänzt, dass ,doch 3 die Hälfte davon [vom markierten Teil der Strecke]“" (38) sei. Philip erwidert, dass ,3“ (39) einem Viertel entspricht, womit er vermutlich meint, dass drei Teile einem Viertel der ganzen Strecke bzw. einem Viertel von zwölf Teilen entsprechen. Daraufhin erwidert Can, dass sie somit vier Teile bzw. „4 Stück anmalen“ (40) müssen, was Philip dann auch in seiner Zeichnung umsetzt. Es fällt auf, dass Can und 
Philip in beiden Teilaufgaben keinerlei Rechnungen oder Brüche notieren, sondern ihre Handlungen ausschließlich auf die ikonische Ebene beschränkt sind.

Insgesamt wird in der Bearbeitung von Can und Philip deutlich, dass sie das Verfahren im Lösungsbeispiel von Beginn der Unterrichtsstunde an nicht erfasst und verstanden haben. Entsprechend interpretieren sie in jeder der nachfolgenden Aufgaben das Vorgehen zum Kürzen von Brüchen auf symbolischer Ebene sowie das Vergröbern der Einteilung neu. In allen Bearbeitungen ist dabei kein stabiles Denkmuster auszumachen, außer dass sie ausschließlich den Nenner des Ausgangsbruch durch den Kürzungsfaktor dividieren. Vor diesem Hintergrund wirkt Philips korrekte Lösung von Aufgabenteil a) augenscheinlich eher zufällig. In seinen Beschreibungen und Erklärungen wird jedoch deutlich, dass diese korrekte Lösung nicht willkürlich oder zufällig zustande kommt, sondern dadurch erklärt werden kann, dass Philip den Vorgaben in der Aufgabenstellung folgt, und genau wie dort formuliert eine neue Einteilung für die Strecke mit halb so vielen Teilen zeichnet, und somit die Einteilung der Strecke korrekt vergröbert. Sein Einzeichnen des korrekten Anteils kann als Anzeichen dafür verstanden werden, dass er das Verfahren nun verstanden hat. Diese Annahme kann jedoch aufgrund seiner Erklärung, dass die Hälfte des Anteils markiert werden müsse, nicht gestützt werden.

Die Bearbeitung von Can und Philip lässt sich in der folgenden Deutungshypothese zusammenfassen:

- Die Verfahren zum Kürzen von Brüchen auf symbolischer Ebene und zum Vergröbern der Einteilung auf anschaulicher Ebene werden nicht übertragen und angewendet. Begonnen mit ihren Antworten auf die fokussierenden Fragestellungen zum Lösungsbeispiel über die Bearbeitung des ersten und zweiten unvollständigen Beispiels bis hin zur dargestellten Bearbeitung der Aufgabe zum Vergröbern der Einteilung einer Strecke ist festzustellen, dass Can und Philip jede Aufgabe auf Grundlage eines neuen Deutungsmusters bearbeiten. Mit Ausnahme, dass sie auf rechnerischer Ebene die Division durch den Kürzungsfaktor nur auf den Nenner des Ausgangsbruchs anwenden, lässt sich in ihren Bearbeitungen und Argumentationen kein stabiles Deutungsmuster rekonstruieren. Ihre Bearbeitungen folgen in allen Fällen spontanen Assoziationen und weisen auf eine Orientierung am Umgang mit natürlichen Zahlen hin, was sich insbesondere darin äußert, dass die Ergebnisse ihrer Kürzungen stets natürliche Zahlen sind, die sie als Anzahl der zu markierenden Teile deuten.

\section{Vergleich der Bearbeitungen zum Vergröbern der Einteilung einer Strecke}

Zusammenfassend zeigen die Analysen der Bearbeitungsprozesse, dass der Repräsentationstransfer des Verfahrens des Vergröberns der Einteilung auf die Darstellung 
in einer Strecke eine besondere Schwierigkeit für die Lernenden darstellt. In keiner Bearbeitung konnte die intendierte Übertragung des anschaulichen Handlungsschemas vollständig rekonstruiert werden. Obgleich der Transfer in dieser Aufgabe im Wesentlichen die anschauliche Ebene betrifft, konnte beobachtet werden, dass dieser auch die Übertragung des rechnerischen Verfahrens zum Kürzen von Brüchen beeinflusst. Vor diesem Hintergrund werden in den dargestellten Bearbeitungsprozessen verschiedene Fehlkonzepte dokumentiert, die auf die fehlerhafte Übertragung des rechnerischen Verfahrens sowie der anschaulichen Handlung zurückgeführt werden können.

Fehlkonzepte in der Übertragung des anschaulichen Vergröberns einer Einteilung: Im Kern der Aufgabe steht die Übertragung des Vergröberns der Einteilung einer Bruchdarstellung, dass zuvor ausschließlich anhand der Flächenrepräsentationen eines Rechtecks und Kreises angewendet wurde auf die lineare Darstellung von Brüchen in einer Strecke. Es fällt auf, dass in keiner der dargestellten Bearbeitungen dieses Verfahren entsprechend der Erläuterung im Lösungsbeispiel und der Beschreibung in den unvollständigen Beispielen fehlerfrei übertragen wurde. Die Fehler der Lernenden bei der Übertragung der anschaulichen Handlung des Vergröberns der Einteilung stellen sich dabei wie folgt dar:

- Das Einzeichnen einer neuen Einteilung mit halb so vielen Teilen wird als Markieren der halben Strecke ohne Veränderung der Einteilung interpretiert (vgl. z. B. Julius, Can).

- Die Länge der ganzen Strecke verändert wird, sodass diese nur noch die halbe bzw. ein Viertel der Länge der ursprünglichen Strecke hat, wobei die Länge der Streckensegmente (der Teile) beibehalten wird (vgl. Marie und Julia).

- Der markierte Anteil der ursprünglichen Darstellung wird in zwei bzw. vier Teile eingeteilt und somit getrennt vom Ganzen betrachtet (vgl. Glen und Johanna).

- Das Einzeichnen einer neuen Einteilung mit einem viertel so viele Teilen wird als Markieren von vier Teilen der ursprünglichen Darstellung interpretiert (vgl. Can und Philip).

Die dokumentierten Fehler können als Fehlkonzepte zum Vergröbern einer Einteilung gesehen werden, da sie ein grundlegendes Missverständnis der wesentlichen Aspekte des Verfahrens suggerieren, (i) dass das Ganze nicht verändert wird, (ii) dass Teile der Einteilung zu einem neuen größeren Teil zusammengefasst werden, und (iii) dass der betrachtete Anteil unverändert bleibt. Alle diese Fehler betreffen fehlerhafte anschauliche Deutungen des Verfahrens. 
Die einzige korrekte Lösung ist in der Bearbeitung von Bennet und Julius dokumentiert. Doch auch hier kann beobachtet werden, dass Bennet das Verfahren nicht direkt aus dem Lesen des Lösungsbeispiels zu Beginn der Unterrichtsstunde oder aus der Bearbeitung der unvollständigen Beispiele überträgt. Da er zunächst keinen Lösungsansatz findet, blättert er zurück zu ihren Lösungen der unvollständigen Beispiele und liest erneut den dritten Teil des Lösungsbeispiels am Computerbildschirm. Erst nach dieser erneuten Reflexion des Vorgehens erkennt stellt er einen Zusammenhang zwischen diesen und der vorliegenden Aufgabe her und überträgt das anschauliche Verfahren durch die Bildung einer Analogie.

Fehler in der Übertragung des rechnerischen Kürzens von Brüchen: Neben den Fehlkonzepten, die bei der Übertragung des anschaulichen Verfahrens rekonstruiert werden konnten, ist zu beobachten, dass auch das rechnerische Verfahren zum Kürzen von Brüchen in vielen Fällen nicht fehlerfrei übertragen wurde.

- Glen und Johanna wenden das Verfahren nur auf den Zähler des ursprünglichen Bruchs an und passen das Rechenverfahren somit an ihre Handlung auf ikonischer Ebene an, indem sie den Nenner des Bruchs beim Kürzen unverändert belassen.

- Philip und Can übertragen ein Fehlkonzept, das bereits in ihren Bearbeitungen der unvollständigen Beispiele dokumentiert ist, und bei dem sie das Ergebnis der Division des Nenners durch den Kürzungsfaktor als Anzahl der zu markierenden Teile des Ganzen interpretieren.

- Bennet und Julius stellen keinen Bezug zum rechnerischen Verfahren her.

Julia und Marie übertragen das rechnerische Verfahren zum Kürzen von Brüchen ohne Fehler. Sie verbinden dieses Verfahren jedoch nicht mit einem Vergröbern der Einteilung, sondern zeichnen eine neue Darstellung, in der die ganze Strecke nur noch halb bzw. ein viertel so lang ist wie in der ursprünglichen Darstellung.

Es sind verschiedene Ursachen für die Schwierigkeiten der Lernenden bei der Übertragung der Verfahren auf symbolischer und anschaulicher Ebene denkbar, die vor allem auf die veränderten Handlungsmöglichkeiten und -Einschränkungen in Bezug auf die Repräsentation in einer Strecke zurückgeführt werden können: Die isolierte Betrachtung des markierten Teils der Strecke liegt bei der eindimensionalen Darstellung von Brüchen in einer Strecke näher als bei der zweidimensionalen Darstellung von Brüchen als Flächeninhalte in einem Rechteck oder einem Kreis. Zudem ist die Transferdistanz zum Lösungsbeispiel und zu den unvollständigen Beispielen durch die grundlegend unterschiedliche äußere Gestaltung der Transferaufgabe besonders groß. Zum einen sind für die einzelnen Lösungsschritte keine gesonderten Arbeitsflächen vorgegeben und zum anderen sind keine Hinweise auf 
die einzelnen Lösungsschritte vorgegeben, wie dies in den Sprechblasen der unvollständigen Beispielen der Fall war.

\subsubsection{Vergleich der Bearbeitungsprozesse in den Transferaufgaben}

Im Vergleich zu den ersten beiden Untersuchungsteilen besteht der Gegenstand des Transfers nicht darin Anteile von verschiedenen Ganzen und Größen zu bestimmen und zu berechnen, sondern darin Brüche durch Kürzen in äquivalente Brüche umzuwandeln und auf anschaulicher Ebene die Einteilung eines Bruchs zu vergröbern. Die intendierte anschauliche Deutung des Rechenverfahrens als Vergröbern der Einteilung baut dabei im Wesentlichen auf der Grundvorstellung von Brüchen als Anteil auf, da das anschauliche Verfahren schließlich auf Anteile angewendet wird. Die Grundidee der Veränderung der Einteilung eines Bruches ist den Lernenden nicht völlig unbekannt, da im vorbereitenden Klassenunterricht das Erweitern von Brüchen als Verfeinern einer Einteilung eingeführt wurde.

Die Analysen der Bearbeitungsprozesse beschreiben deutliche Unterschiede zwischen den intendierten Transferprozessen und den Lösungswegen der Lernenden. Dabei können bereits in der Anwendung des rechnerischen und anschaulichen Verfahrens in den unvollständigen Beispielen zwei Muster in den Bearbeitungen der Lernenden identifiziert werden, die als ursächlich für eine Vielzahl von Schwierigkeiten angenommen werden können, die beim Repräsentationstransfer zum Vergröbern der Einteilung einer Strecke dokumentiert sind. Dabei handelt es sich zum einen um eine Trennung bzw. Entkopplung des rechnerischen Verfahrens und der anschaulichen Handlung und die damit zusammenhängende Fokussierung auf die Anwendung des rechnerischen Verfahrens.

Entkopplung von rechnerischer und anschaulicher Handlung und Fokussierung auf die Anwendung des rechnerischen Verfahrens: Die Entkopplung der rechnerischen und anschaulichen Handlung wird vor allem in dem Bearbeitungsmuster „Kürzen und Darstellen“ deutlich, dass in nahezu allen Bearbeitungen der unvollständigen Beispiele dokumentiert ist. Hierbei kürzen die Lernenden den vorgegebenen Bruchteil zunächst rechnerisch und zeichnen den gekürzten Bruch anschließend in die vorgegebene Repräsentation ein. Für die Repräsentation des gekürzten Bruchteils wird dabei keine Verbindung zu der Darstellung des ungekürzten Bruchteils hergestellt, indem sich die Lernenden an der ursprünglichen Einteilung orientieren und die entsprechende Anzahl von Teilen zu einem neuen Teil zusammenfassen. Stattdessen dokumentieren die Analysen der Bearbeitungs- 
prozesse, dass die Lernenden das Bruchherstellungsverfahren anwenden, um einen „,neuen“ Bruchteil einzuzeichnen. Die anschauliche Deutung als Vergröbern der Einteilung wird nicht übertragen und angewendet.

Im Gegensatz zu der anschaulichen Deutung beschreiben die Analysen die Bearbeitungsprozesse der Lernenden eine Fokussierung auf die Anwendung des rechnerischen Verfahrens, das in allen Fällen - wenn auch in einigen Fällen fehlerhaft - übertragen wird. In diesem Zusammenhang ist anzunehmen, dass die Division von Zähler und Nenner bzw. die Division natürlicher Zahlen für die Lernenden ein vertrautes Handlungskonzept darstellt, wohingegen die Transformation einer Darstellung zunächst sehr abstrakt und eine mentale Simulation der Vergröberungshandlung eine besondere Anforderung darstellen. Obwohl die Lernenden mit wenigen Ausnahmen die zentralen Aspekte des anschaulichen Handlungskonzepts in ihren Antworten auf die fokussierenden Fragestellungen zum Lösungsbeispiel formuliert haben, dokumentieren die Bearbeitungen der unvollständigen Beispiele einen ausbleibenden Transfer. Da die Lernenden zur Beantwortung der fokussierenden Fragestellungen sich an den Darstellungen und Erläuterungen im Lösungsbeispiel orientieren konnten, ist diese „Vorlage“ in den unvollständigen Beispielen nicht gegeben, sodass sie sich das Vergröbern der Einteilung zunächst vorstellen müssten. Aus diesem Grund ist anzunehmen, dass sie sich in ihren Lösungen an dem ihnen vertrauten Handlungskonzepten „Division von natürlichen Zahlen“ und „Anwendung des Bruchherstellungsverfahrens“ orientieren.

Die beobachtete Fokussierung auf die Übertragung des rechnerischen Verfahrens und die Entkopplung der Handlungen auf rechnerischer und anschaulicher Ebene stellt einen plausiblen Erklärungsansatz für die vielfältigen Fehlkonzepte, die in den Bearbeitungsprozessen zum Vergröbern der Einteilung einer Strecke dokumentiert sind.

Entwicklung von Fehlkonzepten zum anschaulichen und rechnerischen Verfahren: Die Bearbeitungen der Lernenden zum Vergröbern der Einteilung einer Strecke sind durch eine Vielfalt verschiedener fehlerhafter Deutungen des anschaulichen Verfahrens charakterisiert. In den Bearbeitungen der Lernenden ist dokumentiert, dass

- das Einzeichnen einer neuen Einteilung mit halb so vielen Teilen als Markieren der halben Strecke ohne Veränderung der Einteilung interpretiert wird (vgl. z. B. Julius, Can),

- die Länge der ganzen Strecke verändert wird, sodass diese nur noch die halbe bzw. ein Viertel der Länge der ursprünglichen Strecke hat, wobei die Länge der Streckensegmente (der Teile) beibehalten wird (vgl. Marie und Julia), 
- der markierte Anteil der ursprünglichen Darstellung in zwei bzw. vier Teile eingeteilt und somit getrennt vom Ganzen betrachtet wird (vgl. Glen und Johanna) und

- das Einzeichnen einer neuen Einteilung mit einem viertel so viele Teilen als Markieren von vier Teilen der ursprünglichen Darstellung interpretiert wird (vgl. Can und Philip).

Da diese fehlerhaften Lösungen und Lösungsansätze ein grundlegendes Missverständnis der wesentlichen Aspekte des anschaulichen Verfahrens suggerieren, können sie als Fehlkonzepte bezeichnet werden. Die Lernenden übertragen nicht ihre Beobachtungen aus dem Lösungsbeispiel, (i) dass das Ganze nicht verändert wird, (ii) dass Teile der Einteilung zu einem neuen größeren Teil zusammengefasst werden, und (iii) dass der betrachtete Anteil unverändert bleibt, sondern deuten das Verfahren in der Transfersituation neu. Vor dem Hintergrund der Beobachtungen aus den Bearbeitungen der unvollständigen Beispiele kann diese neue Deutungsaushandlung der Lernenden auf eine unzureichende Verknüpfung des rechnerischen und des anschaulichen Verfahrens zurückgeführt werden. Das Hervortreten von Fehlkonzepten des anschaulichen Verfahrens wird insbesondere dadurch bestärkt, dass die Formulierung der Aufgabenstellung den Schwerpunkt auf die Anwendung des anschaulichen Verfahrens setzt. Anders als in den unvollständigen Beispielen soll hier zunächst eine neue Einteilung eingezeichnet werden und erst im Anschluss daran das Vorgehen auf die symbolische Ebene übersetzt werden. Dies hat zur Folge, dass die Lernenden nicht das Bearbeitungsmuster „Kürzen und Darstellen“ anwenden, sondern ihren Fokus auf die Veränderung der Einteilung der Strecke legen.

In einigen Fällen konnte zudem beobachtet werden, dass die Lernenden zur Beschreibung des Vergröberns auf symbolischer Ebene nicht das Verfahren zum Kürzen übertragen, sondern ihre fehlerhafte anschauliche Handlung auf die symbolische Ebene übersetzen. $\mathrm{Zu}$ nennen ist hierbei insbesondere die Lösung von Glen und Johanna, die in ihrer anschaulichen Darstellung die Einteilung der Strecke beibehalten und lediglich den markierten Anteil neu einteilen. Sie übersetzen dieses Verfahren entsprechend so, dass sie nur den Zähler des Bruchs dividieren und den Nenner beibehalten. Ihre Lösung dokumentiert, wie das rechnerische Verfahren, dass sie zuvor wiederholt korrekt angewendet haben, auf Grundlage eines Fehlkonzepts auf anschaulicher Ebene verändert wird.

Weitere Schwierigkeiten beim Transfer des Verfahrens auf eine neue Repräsentation: Neben der Entwicklung von Fehlkonzepten dokumentieren die Bearbeitungen der Lernenden zudem die besonderen Schwierigkeiten, die beim 
Transfer des Verfahrens auf eine neue Repräsentation auftreten können. Diese sind nicht nur auf den Transfer auf die Strecke beschränkt, sondern sind auch beim Repräsentationswechsel im zweiten unvollständigen Beispiel zu erkennen. Diese umfassen:

- Fehlerhafte Assoziationen, wie zum Beispiel, dass das Einzeichnen einer neuen Einteilung mit halb so vielen Teilen als Markieren von halb so vielen Teilen gedeutet wird,

- die Verwechslung von Vergröbern und Verfeinern einer Einteilung und

- Schwierigkeiten beim Darstellen von Bruchteilen in einer Kreisrepräsentation.

Auch diese Fehler und Schwierigkeiten der Lernenden betreffen vorwiegend die anschauliche Deutung des Verfahrens.

Zusammenfassend kann festgehalten werden, dass die beobachtete Entkopplung des rechnerischen Verfahrens und der anschaulichen Deutung des Verfahrens beim Transfer im Rahmen der Anwendung der Verfahren auf neue Brüche und neue Repräsentationen dazu führt, dass das Verfahren auf anschaulicher Ebene neu gedeutet wird. Dabei kommt es in vielen Fällen zu Fehldeutungen des Verfahrens, die das Potenzial haben sich zu stabilen Fehlkonzepten und Fehlvorstellungen zu entwickeln. Es konnte zudem beobachtet werden, dass die Möglichkeit besteht, dass diese Fehlkonzepte und -Vorstellungen des anschaulichen Verfahrens auf das rechnerische Verfahren übertragen werden, und dieses entsprechend in einem negativen Transfer angepasst wird.

\subsection{Vergleich und Diskussion der deskriptiven Analysen}

Im Folgenden werden die Ergebnisse der Analysen der Bearbeitungsprozesse zusammengeführt. Im Vordergrund dieser Zusammenführung steht der Vergleich der Ergebnisse der Analysen der drei videographierten Unterrichtsstunden mit dem Ziel Gemeinsamkeiten und Unterschiede herauszuarbeiten und vor dem Hintergrund der formulierten Forschungsfragen zu diskutieren.

\subsubsection{Zusammenführung der Ergebnisse}

In diesem Abschnitt werden die Ergebnisse der Analysen der Bearbeitungsprozesse zusammengefasst und miteinander in Beziehung gesetzt, um Gemeinsamkeiten und 
Unterschiede der Analysen der Bearbeitungen zu den drei inhaltlichen Schwerpunkten herauszustellen.

\section{Intendierte Transferprozesse}

In Vorbereitung der deskriptiven Analysen der Bearbeitungsprozesse der Lernenden wurden auf sachanalytischer Ebene die Aufgaben auf intendierte Transferprozesse und notwendige Grundvorstellungen hin untersucht. Im Fokus stand hierbei, dass der Hauptgegenstand der Transferprozesse in allen Fällen ein Verfahren ist, das auf neue Zahlenwerte, eine neue Repräsentation oder einen neuen Sachkontext angewendet werden soll:

- Das Bruchherstellungsverfahren: Teilen eines Ganzen in $n$ gleiche Teile und $m$-faches Vervielfachen eines Teils,

- Berechnen von Anteilen beliebiger Größen: Teilen einer Größe in $n$ gleiche Teile und $m$-faches Vervielfachen eines Teils,

- Kürzen von Brüchen: Division von Zähler und Nenner eines Bruchs $\frac{m}{n}$ durch den gleichen Divisor $k$.

Beim Transfer eines Verfahrens oder Handlungskonzepts auf eine neue Repräsentation bestehen die zentralen Transferschritte im Anpassen des Handlungskonzepts an die Eigenschaften des neuen Repräsentationsobjekts. Mit Bezug auf den Transfer eines Verfahrens auf eine neue Sachsituation stellt insbesondere die Identifizierung der Bezugsgrößen und die Analogiebildung zwischen den einzelnen Handlungsschritten die wichtigsten Transferschritte dar.

Aus der Sicht des Grundvorstellungskonzepts ist jedes rechnerische Verfahren mit einem anschaulichen Handlungskonzept verbunden, das in den für die Analysen der Bearbeitungsprozesse ausgewählten Aufgaben ebenfalls als Gegenstand von Transferprozessen übertragen werden sollte. Der Transfer besteht dabei in der Übertragung und Anwendung der anschaulichen Handlungskonzepte auf neue Repräsentationen und Sachkontexte:

- Bruchherstellungsverfahren und Berechnen von Anteilen beliebiger Größen: Teilen eines Ganzen (Figur, Repräsentant einer Größe) in $n$ kongruente Teile und Markieren von $m$ Teilen,

- Kürzen von Brüchen: Vergröbern der Einteilung einer Bruchdarstellung.

In Hinsicht auf die Ausbildung und Entwicklung von Grundvorstellungen besteht ein wesentlicher Transferprozess in der Verknüpfung der rechnerischen und anschaulichen Handlungskonzepte, d.h. der Verknüpfung der Grundvorstellung Bruch als 
Anteil mit der Grundvorstellung Bruch als Operator sowie die Verknüpfung des Kürzens von Brüchen mit dem Vergröbern einer Einteilung auf anschaulicher Ebene.

\section{Vergleich der intendierten Transferprozesse mit den in den Bearbeitungspro- zessen der Lernenden dokumentierten Transferprozesse}

In den deskriptiven Analysen der Bearbeitungsprozesse können in allen Fällen Transferprozesse rekonstruiert werden. Während eine überwiegende Mehrheit der rekonstruierten Transferprozesse den intendierten Transferprozessen entspricht, sind in vielen Fällen deutliche Divergenzen zu intendierten Transferprozessen zu erkennen.

\section{Transferprozesse bei der Übertragung und Anwendung des Bruchherstellung-} sverfahrens: Die Analysen der Bearbeitungsprozesse dokumentieren, dass das Operatorschema zur Herstellung von Brüchen in vielen Fällen übertragen und auf neue Brüche und Repräsentationen angewendet werden konnte. Hierbei zeigte sich, dass die Lernenden vor allem das rechnerische Handlungsmuster : $n \cdot m$ auf symbolischer Ebene übertragen und anwenden. Auf anschaulicher Ebene hingegen konnten deutliche Divergenzen zwischen den intendierten und dokumentierten Transferprozessen festgestellt werden. Diese betreffen insbesondere die anschauliche Deutung der Herstellungsoperatoren. Es konnte beobachtet werden, dass vor allem der Herstellungsoperator $\cdot m$ nicht als $m$-faches Vervielfachen, sondern als Subtraktion zum Wegnehmen von $m$ Teilen oder als Addition zum Hinzufügen von $m$ Teilen gedeutet wurde. In den Bearbeitungen der Lernenden führten diese fehlerhaften Deutungen insbesondere zu Schwierigkeiten und Fehlern bei der ikonischen Darstellung von Brüchen. In einem Fall, indem das rechnerische Handlungsschema nicht übertragen und angewendet wurde, konnte beobachtet werden, wie die Lernenden anhand der vorgegebenen Darstellungen der anschaulichen Bruchherstellung die Herstellungsoperatoren neu hergeleitet haben (vgl. 5.1, Can, Philip \& Glen). Auch in diesem Fall kamen fehlerhafte Operationsvorstellungen zum Tragen, die in den Lösungen der Lernenden durch die Wahl der falschen Rechenoperationen deutlich wurden.

Beim Repräsentationstransfer des Herstellungsverfahrens bzw. der anschaulichen Herstellungshandlung auf neue Repräsentationsobjekte, konnte beobachtet werden, dass die Teiloperatoren der Herstellung in den meisten Fällen übertragen und an die Eigenschaften des neuen Repräsentationsobjekts angepasst werden konnten. In zwei Fällen konnte hierbei ein Ausbleiben des Transfers der Herstellungshandlung beobachtet werden. In einem dieser Fälle wurde der darzustellende Bruch $\frac{m}{n}$ zwar korrekt als Anteil $m$ von $n$ Teilen gedeutet, konnte jedoch nicht in eine Herstellungshandlung übersetzt werden, bei der das Ganze zunächst in $n$ Teile 
geteilt wird, von denen $m$ Teile markiert werden. Es wurde eine statisch-abbildhafte Vorstellung übertragen, die nicht mit einer dynamischen Vorstellung der Herstellung verknüpft wurde und somit zu einem fehlerhaften Lösungsansatz führte (vgl. 5.1, Aliya \& Aisha). In einem zweiten Fall konnte beobachtet werden, dass nur der erste Teiloperator zum Einteilen des Ganzen auf die Darstellung von Brüchen an einer Strecke übertragen wurde. Hierbei vernachlässigten die Lernenden die Zähler der darzustellenden Brüche $\frac{m}{n}$ und interpretierten diese als $\frac{1}{n}$, das $n$-mal in das Ganze hinein passen muss (vgl. 5.1, Luca \& Miguel). Auch in diesem Fall wurde keine dynamische Vorstellung der Herstellung, sondern eine statisch-abbildhafte Vorstellung übertragen und angewendet.

Transferprozesse beim Berechnen von Anteilen beliebiger Größen: In den Analysen der Bearbeitungsprozesse zum Transfer des Operatorschemas zur Berechnung von Anteilen beliebiger Größen ist festzustellen, dass die Lernenden das Verfahren in allen Fällen übertragen und anwenden. Dabei scheint die Beschaffenheit des Ganzen in keinem Fall von Bedeutung zu sein. Unabhängig davon, ob das Ganze eine Figur, eine Maßzahl oder eine diskrete Menge von Objekten ist, versuchen sie das Operatorschema anzuwenden. In den Aufgaben, in denen die Anteile in der Form $\frac{m}{n}$ von $G$, z. B. $\frac{4}{7}$ von $21000 €$, vorgegeben sind, wenden die Lernenden das Verfahren zumeist spontan und ohne erkennbare Schwierigkeiten an.

Die Hauptfehlerquelle und größte Schwierigkeit der Lernenden beim Transfer der Anteilberechnung von beliebigen Größen betrifft die Anwendung in einem geänderten oder neuen Sachzusammenhang. Bereits in sehr nahen Transferaufgaben, in denen der Sachkontext unverändert ist und die Aufgabe sich lediglich in den Zahlenwerten vom Lösungsbeispiel unterscheidet, sind deutliche Schwierigkeiten bezüglich der Analogiebildung zwischen dem Lösungsbeispiel und dem ersten unvollständigen Lösungsbeispiel zu erkennen. Diese betreffen jedoch nicht das rechnerische Verfahren, sondern das Identifizieren der zu berechnenden Anteile im Sachkontext.

In Aufgaben mit geänderten Bezugsgrößen und einem anderen bzw. neuen Sachkontext ist in vielen Fällen zu beobachten, dass die Analogiebildung nicht gelingt, weil die Lernenden fehlerhafte Aufteilungen des Ganzen vornehmen. Der Transfer des Lösungsweges aus dem Lösungsbeispiel scheitert dabei zumeist an der Identifizierung des Ganzen und der Grundlage für die Anteilbildung, sodass sie den notwendigen Transfer des Lösungswegs nicht oder nur fehlerhaft vornehmen können.

Auch in den Bearbeitungen der mehrschrittigen Transferaufgabe sind keine Schwierigkeiten beim Transfer des Operatorschemas zu erkennen und es gelingt allen Lernenden Anteile einer diskreten Menge zu berechnen. Es gelingt vielen 
Lernenden jedoch nicht, die vorgegebenen Größen und Anteile im Sachzusammenhang einzuordnen und die Beziehungen zwischen Anteil, Teil und Ganzem herzustellen, sodass ihre Berechnungen in vielen Fällen auf falschen Werten beruhen. Die Hauptschwierigkeit und Fehlerquelle in dieser Aufgabe ist die Strukturierung der Situation und die korrekte Bestimmung der zu berechnenden Anteile.

Im Lösungsbeispiel sowie im ersten unvollständigen Beispiel wird die Berechnung des Anteils in einem Pfeilschema dargestellt, in der die Rechnung strukturiert veranschaulicht wird. Die Analysen der Bearbeitungsprozesse der Lernenden zeigen, dass die Darstellung der Rechnung in einem Pfeilschema von vielen Lernenden übertragen wird. Dies betrifft auch Fälle, in denen die Lernenden das Pfeilschema nicht für die Notation ihrer Rechnung nutzen, sondern lediglich feststellen, dass sie „wie in diesem Pfeilschema rechnen“ müssen. Obgleich dieses in vielen Fällen erkannt wird, wird es nicht benutzt.

Transfer beim Kürzen von Brüchen als Vergröbern einer Einteilung: In den Bearbeitungen der Aufgaben zum Kürzen von Brüchen als Vergröbern einer Einteilung ist festzustellen, dass die Lernenden vor allem das rechnerische Verfahren der Division von Zähler und Nenner durch einen gemeinsamen Faktor übertragen und anwenden. Der Transfer des rechnerischen Verfahrens - wenn auch in einigen Fällen fehlerhaft - ist in allen dargestellten Bearbeitungen dokumentiert.

Im Gegensatz zum Transfer des rechnerischen Verfahrens ist mit Blick auf den Transfer der anschaulichen Deutung als Vergröbern der Einteilung festzustellen, dass diese nur selten und in den meisten Fällen fehlerhaft übertragen wird. Insbesondere in den Bearbeitungen der unvollständigen Beispielen konnte rekonstruiert werden, dass die Lernenden nicht die Einteilung der vorgegebenen Bruchdarstellung verfeinern, sondern den Bruch auf symbolischer Ebene kürzen und zur Darstellung des neuen Bruchs das Bruchherstellungsverfahren übertragen und anwenden und somit eine „neue“ Darstellung des gekürzten Bruchs anfertigen ohne eine Beziehung zur Darstellung des Ausgangsbruchs herzustellen.

In den Aufgaben zum Repräsentationstransfer des anschaulichen Verfahrens sind zahlreiche Fehler dokumentiert. Diese bestehen vor allem in fehlerhaften Assoziationen, bei denen zum Beispiel das Einzeichnen einer Einteilung mit halb so vielen Teilen als Markieren der Hälfte der Teile interpretiert wird, der Verwechslung mit dem Erweitern von Brüchen als Verfeinern einer Einteilung sowie Schwierigkeiten bei der Anwendung des Bruchherstellungsverfahrens bei der ikonischen Darstellung von Brüchen. Alle diese Fehler betreffen die anschauliche Deutung des Verfahrens. 


\subsubsection{Vergleich und Diskussion der Ergebnisse der deskriptiven Analysen}

Ungeachtet der unterschiedlichen inhaltlichen Schwerpunkte der drei Datenerhebungen lassen sich im Vergleich der Analysen der Bearbeitungsprozesse der Lernenden Gemeinsamkeiten und Unterschiede identifizieren, die eine Erklärungsgrundlage für die dokumentierten Divergenzen zwischen den intendierten und den deskriptiv erfassten Transferprozessen bieten. Diese betreffen insbesondere die Entwicklung sachadäquater Vorstellungen und Handlungskonzepte zu den behandelten Inhalten.

\section{Entkopplung der rechnerischen und anschaulichen Handlungskonzepte}

Die zentrale Gemeinsamkeit kann als Entkopplung der rechnerischen und anschaulichen Handlungskonzepte beschrieben werden. In den Transferprozessen ist diese Entkopplung vor allem darin zu sehen, dass die Lernenden in fast allen Fällen die rechnerischen Verfahren auf neue Aufgaben übertragen, während der Transfer des entsprechenden anschaulichen Handlungskonzepts in vielen Fällen ausbleibt oder fehlerbehaftet ist.

In der ersten Datenerhebung zum Transfer des Bruchherstellungsverfahrens ist festzustellen, dass die Verknüpfung der rechnerischen und anschaulichen Herstellungshandlung stark von individuellen Operationsvorstellungen sowie von individuellen Deutungen der anschaulichen Herstellungshandlung zur Darstellung von Brüchen beeinflusst wird. In den Bearbeitungen von einigen Lernenden konnte rekonstruiert werden, dass sie, ungeachtet einer korrekten Übertragung des rechnerischen Verfahrens, die anschaulichen Herstellungsschritte isoliert betrachten und mit fehlerhaften Rechenoperationen verbinden. Diese äußern sich explizit in den Übersetzungen der Teiloperatoren als Subtraktion oder Addition anstelle der Übersetzung als Division und Multiplikation.

In den Analysen der zweiten und dritten Datenerhebung zum Berechnen von Anteilen beliebiger Größen und zum Kürzen von Brüchen als Vergröbern einer Einteilung treten die Kennzeichen für eine Trennung der rechnerischen Verfahren deutlicher hervor. In den Bearbeitungen der Lernenden ist eine zunehmende Fokussierung auf die Anwendung des rechnerischen Verfahrens festzustellen. Diese äußert sich in den Bearbeitungen zur Berechnung von Anteilen beliebiger Größen insbesondere darin, dass die Lernenden die in den Aufgabenstellungen geschilderten Sachsituationen in einigen Fällen nicht hinreichend verarbeiten und strukturieren, sondern anhand der enthaltenen Zahlenwerte direkt eine Rechnung aufstellen, ohne einen inhaltlichen Bezug zur Sachsituation herzustellen. In diesem Zusammenhang 
beschränken sich die Argumentationen der Lernenden auf die symbolische bzw. rechnerische Ebene und es werden nur selten anschauliche Bezüge hergestellt.

In den Bearbeitungen zum Transfer des Kürzens von Brüchen tritt die Entkopplung des rechnerischen und anschaulichen Handlungskonzepts besonders deutlich in Erscheinung. Nur in wenigen Fällen ist zu erkennen, dass die Lernenden mit dem Kürzen von Brüchen das anschauliche Verfahren des Vergröberns einer Einteilung verbinden und übertragen. Stattdessen ist in nahezu allen Bearbeitungen der Lernenden das Bearbeitungsmuster „Kürzen und Darstellen“ zu beobachten, bei dem die Lernenden den ursprünglichen Bruch zunächst auf rechnerischer Ebene kürzen und den gekürzten Bruch anschließend auf Grundlage des Bruchherstellungsverfahrens ikonisch darstellen. Weiterhin ist festzustellen, dass in vielen Fällen keine Beziehung zwischen den ikonischen Bruchdarstellungen des ungekürzten und des gekürzten Bruchs hergestellt wird, die ikonischen Bruchdarstellungen als Darstellungen unterschiedlicher Brüche betrachtet werden, und somit die Äquivalenz der beiden Brüche nicht erkannt wird. Die Fokussierung auf die Anwendung des rechnerischen Verfahrens und der damit verbundene ausbleibende Transfer des anschaulichen Handlungskonzepts führt besonders beim Repräsentationstransfer auf ikonischer Ebene zu neuen und in vielen Fällen fehlerhaften Deutungen des anschaulichen Handlungskonzepts. In den Bearbeitungen der Lernenden zum Transfer auf das Vergröbern der Einteilung einer Strecke konnten in diesem Zusammenhang eine Vielfalt fehlerhafter Deutungen der Lernenden rekonstruiert werden, die das Potenzial haben zu robusten Fehlvorstellungen entwickelt zu werden. Mit Bezug auf die Entkopplung der symbolischen und anschaulichen Ebene fällt insbesondere beim Repräsentationstransfer auf die Strecke auf, dass die Lernenden in Situationen, in denen sie das anschauliche Verfahren nicht übertragen und keinen spontanen Lösungsansatz haben, nicht in Betracht ziehen, die dargestellten Brüche auf rechnerischer Ebene zu kürzen und die gekürzten Brüche einzeichnen.

Über den Verlauf der Datenerhebungen stellt sich die Entkopplung der symbolischen und anschaulichen Handlungskonzepte als Entwicklung dar, die mit fortschreitendem Verlauf der Unterrichtseinheit deutlicher in Erscheinung tritt. Während zu Beginn der Unterrichtseinheit nur in wenigen Fällen eine getrennte Verarbeitung der rechnerischen und anschaulichen Handlungskonzepte dokumentiert sind, ist dieses Phänomen am Ende der Unterrichtseinheit in nahezu allen Bearbeitungen der Lernenden zu identifizieren. Diese Entwicklung kann durch die Fokussierung auf die Anwendung von Rechenverfahren begründet werden, die für die Lernenden in den meisten Fällen einen wesentlich geringeren Aufwand bedeuten und im Fall von gering entwickelten anschaulichen Vorstellungen zunächst weniger fehleranfällig sind. Mit zunehmendem Verlauf der Unterrichtseinheit und zunehmender Komplexität und Abstraktion der Handlungskonzepte sowie beim Transfer auf neue 
Repräsentationen und Sachkontexte führt der fehlende anschauliche Bezug jedoch zunehmend zu Fehlern, die in Beziehung zu verschiedenen in der Literatur beschriebenen Fehlermustern und Fehlkonzepten stehen.

\section{Entwicklung von Fehlkonzepten aufgrund von ausbleibendem und negati- vem Transfer}

Die in den Bearbeitungsprozessen der Lernenden identifizierten Fehlkonzepte und Fehlvorstellungen können in vielen Fällen auf die Entkopplung der symbolischen und anschaulichen Handlungskonzepte zurückgeführt werden. Entsprechend der zuvor beschriebenen Fokussierung auf die Anwendung rechnerischer Verfahren bleibt ein Transfer des anschaulichen Handlungskonzepts in vielen dieser Fälle aus und die Lernenden entwickeln neue individuelle anschauliche Deutungen der rechnerischen Verfahren.

In den Analysen der Bearbeitungen der ersten Datenerhebung erweist sich die Verbindung der Grundvorstellung Bruch als Anteil mit dem Bruchherstellungsverfahren als zentraler Transferprozess. Es wird erwartet, dass die Lernenden die statische Vorstellung von Brüchen als Teile eines Ganzen mit der dynamischen Vorstellung der Bruchherstellung verbinden. Die Verknüpfung dieser Grundvorstellungen ist besonders beim Wechsel innerhalb und zwischen Repräsentationsebenen und Bruchdarstellungen (Repräsentationstransfer) erforderlich und steht somit in einem engen Zusammenhang mit dem Aufbau tragfähiger und flexibler Bruchzahlvorstellungen (vgl. Abschnitt 2.2.3). Vor diesem Hintergrund konnte insbesondere in den Bearbeitungsprozessen der Lernenden zum Transfer des Bruchherstellungsverfahrens auf die Darstellung in einer Strecke festgestellt werden, dass die Dominanz der Anteilvorstellung eher fehlerführend ist und eine komponentenweise Betrachtung von Brüchen unterstützen kann.

Die Orientierung am Umgang mit natürlichen Zahlen konnte überdies in weiteren Bearbeitungsprozessen festgestellt werden. In diesen Fällen konnte insbesondere eine komponentenweise Betrachtung von Zähler und Nenner beim Transfer der Anteilberechnung auf einen neuen Sachkontext sowie beim Transfer des Verfahrens zum Vergröbern einer Einteilung dokumentiert werden. In diesen Fällen ist der Hintergrund der Orientierung am Umgang mit natürlichen Zahlen jedoch nicht auf eine unzureichende Verknüpfung der Anteil- und Operatorvorstellung, sondern im ausbleibenden Transfer von anschaulichen Handlungskonzepten und der Fokussierung auf die Anwendung von rechnerischen Verfahren zu vermuten.

Beim Transfer des Verfahrens zur Berechnung von Anteilen in einem komplexen Sachkontext konnte in einem Fall beobachtet werden, dass der Bruch nicht als relativer Anteil eines Ganzen, sondern als absoluter Anteil im Sinne der Anzahl der 
Objekte einer diskreten Menge interpretiert wurde (vgl. 5.2, Aufgabe 9, Bearbeitung von Julia \& Marie).

Wesentlich deutlicher und zahlreicher ist die Orientierung an der Ordnungsstruktur der natürlichen Zahlen in den fehlerhaften anschaulichen Deutungen des Kürzens von Brüchen beschrieben. In all diesen Fällen bleibt der Transfer des anschaulichen Verfahrens aus und es wird eine neue Deutung des Einzeichnens einer neuen Einteilung mit einem $n$-tel so vielen Teilen entwickelt. Der Hintergrund dieses ausbleibenden Transfers ist in der getrennten Verarbeitung des rechnerischen und anschaulichen Verfahrens zu vermuten, wobei in den meisten Fällen anzunehmen ist, dass durch das Lesen eines dreiteiligen Lösungsbeispiels und der Bearbeitung zweier unvollständiger Beispiele kein entsprechendes Handlungskonzept aufgebaut wurde (vgl. Bearbeitungsmuster „Kürzen und Darstellen“).

Des Weiteren konnte in den Bearbeitungen der ersten Datenerhebung sowie in vereinzelten Fällen der zweiten und dritten Datenerhebung die wörtliche Übertragung sprachlicher Formulierungen identifiziert werden. Hierbei wird die wörtliche Beschreibung einer Handlung mit konkreten Gegenständen auf die symbolische Ebene übersetzt und entsprechend mit Rechenoperationen verbunden, die bezogen auf den Umgang mit realen Gegenständen durchaus adäquat sind (Subtraktion als Wegnehmen und Addition als Hinzufügen), jedoch nicht den intendierten Deutungen der Division als Teilen eines Ganzen und der Multiplikation als Vervielfachen eines Teils entsprechen.

\section{Aktivierung und Koordination von Vorwissen}

Ausbleibende und fehlerhafte Übertragungen konnten insbesondere in Aufgaben beschrieben werden, in denen ein Verfahren auf neue Darstellungen und Sachkontexte übertragen werden sollte, wodurch eine Anpassung der entsprechenden Handlungskonzepte an die Eigenschaften der neuen Repräsentation oder der neuen Sachsituation notwendig ist. In diesen Situationen ist von besonderer Bedeutung über welches Vorwissen die Lernenden verfügen und inwieweit sie dieses aktivieren können.

Vor diesem Hintergrund können in den Bearbeitungen der Lernenden drei Transfersituationen unterschieden werden, in denen die Aktivierung von Vorwissen von Bedeutung ist: Der Analogiebildung, dem Transfer zwischen Darstellungen und dem Transfer zwischen Sachkontexten.

Transfer durch Analogiebildung: Die unvollständigen Beispiele der drei Datenerhebung sind jeweils strukturgleich zum Lösungsbeispiel konzipiert. Das erste unvollständige Beispiel unterscheidet sich in allen Fällen allein in den Zahlenwerten vom Lösungsbeispiel, wohingegen im zweiten Lösungsbeispiel neben einer Ände- 
rung der Zahlenwerte auch eine Variation der enthaltenen ikonischen Repräsentationen oder des Sachkontexts zu berücksichtigen ist. Somit erfordert die Lösung der unvollständigen Beispielen in allen Fällen die Bildung einer Analogie zum Lösungsbeispiel, die als Voraussetzung für einen Transfer der einzelnen Bearbeitungsschritte betrachtet werden kann. Der Transfer der Lösungsschritte aus dem Lösungsbeispiel wird in beiden unvollständigen Beispielen durch Hinweise in Sprechblasen unterstützt.

In den Analysen der Bearbeitungsprozesse lassen sich drei Aspekte charakterisieren, die einen deutlichen Einfluss auf die Analogiebildung zum Lösungsbeispiel haben: (i) Die Verarbeitung des Lösungsbeispiels, (ii) die äußere Gestaltung des unvollständigen Beispiels und (iii) Unterschiede auf Ebene der ikonischen Darstellung und des Sachkontexts.

Während die meisten Schülerpaare sich eingehend mit dem Lösungsbeispiel beschäftigt haben, indem sie sich gegenseitig die einzelnen Lösungsschritte erklärt und aufkommende Fragen beim Lesen des Lösungsbeispiels und der fokussierenden Fragen zum Lösungsbeispiel gemeinsam beantwortet haben, konnte in einigen Fällen auch beobachtet werden, dass die Lernenden das Lösungsbeispiel lediglich überflogen und kurz ,durchgeklickt“ haben, ohne darüber zu sprechen. In diesen Fällen wurden die Verfahren und Handlungskonzepte aus dem Lösungsbeispiel nicht in der Bearbeitung der unvollständigen Beispiele angewendet. Womöglich aufgrund eines fehlenden Ausgangspunkts für die Abbildung der Lösungsschritte versuchten die Lernenden die einzelnen Lösungsschritte anhand der Lösungshinweise im unvollständigen Beispiel herzuleiten. Die intendierten Transferprozesse blieben hierbei zumeist aus und es konnten wiederholt fehlerhafte Deutungen der rechnerischen sowie insbesondere der anschaulichen Handlungskonzepte rekonstruiert werden.

Jedoch auch in den Fällen, in denen die Lernenden die einführenden Lösungsbeispiele eingehend gelesen und die fokussierenden Fragestellungen korrekt beantwortet und dabei die zentralen inhaltlichen Aspekte der Aufgabenlösung notiert haben, konnte festgestellt werden, dass bereits der Transfer des Lösungsverfahrens auf das strukturell isomorphe erste unvollständigen Beispiel zu Schwierigkeiten führte und der intendierte Transfer zunächst ausblieb, da keine Verbindung zum Lösungsbeispiel hergestellt wurde. Die Ursache dafür kann in den Unterschieden der Oberflächenmerkmale der ersten unvollständigen Beispiele und der Lösungsbeispiele vermutet werden. Während die Lösungen in den Lösungsbeispielen zumeist verschiedene Abbildungen und Visualisierungen zur Veranschaulichung, wie zum Beispiel schematische Zeichnungen von Familien mit allen Familienmitgliedern, enthielten, wurden diese in der Gestaltung der unvollständigen Beispiele nicht übernommen. Der ausbleibende Transfer kann in diesen Fällen über die Orientierung an den Oberflächenmerkmalen der Aufgaben begründet werden. Dieses Phänomen 
ist vor allem in den Bearbeitungen der zweiten Datenhebung dokumentiert, in dem die Aufgaben in einen Sachkontext eingebunden waren, der in diesem Zusammenhang eine Analogiebildung zwischen den strukturellen Merkmalen der Aufgaben erschwert. Während nur in vereinzelten Bearbeitungen Schwierigkeiten bei der Analogiebildung zwischen dem Lösungsbeispiel und dem ersten unvollständigen Beispiel beobachtet werden konnten, treten diese in den Bearbeitungen der zweiten unvollständigen Beispiele deutlicher hervor, da diese zudem eine Änderung auf Ebene des Repräsentationsobjekts oder des Sachkontexts enthielten. Dies führte in vielen Fällen dazu, dass die Lernenden Schwierigkeiten bei der Wahl der Bezugsgröße zur Bestimmung der Anteile hatten.

Insgesamt ist in den Bearbeitungen der unvollständigen Beispiele festzustellen, dass die äußere Gestaltung der unvollständigen Beispiele sowie Änderungen der Darstellungen und des Sachkontexts in vielen Fällen dazu führen, dass die Lernenden keine Verbindung zum Lösungsbeispiel herstellen und die Struktur der Aufgabenlösung nicht korrekt abbilden. Es können entsprechend nicht die erforderlichen Erfahrungsbereiche aktiviert und miteinander koordiniert werden. Dies kann somit einerseits damit erklärt werden, dass kein entsprechender Erfahrungsbereich aufgebaut wurde, oder dass aufgrund unterschiedlicher Oberflächen- und Strukturmerkmale der Aufgaben keine Verbindung zwischen diesen hergestellt werden kann.

Transfer zwischen Darstellungen: In vielen Fällen bestand der intendierte Transfer in den dargestellten Bearbeitungen von Transferaufgaben in der Übertragung eines anschaulichen Verfahrens auf ein neues Repräsentationsobjekt. Hierbei konnte beobachtet werden, dass ein zentrales Kriterium für einen erfolgreichen Transfer auf eine neue Repräsentation die Aktivierung der Handlungskonzepte zum Umgang mit dem neuen Repräsentationsobjekt ist. Diese hängen im Wesentlichen davon ab, welche Größen sie repräsentieren und welche Handlungskonzepte mit diesen Repräsentationsobjekten verbunden sind. Dies betrifft vor allem die Bruchdarstellung in einem Kreis oder einem Rechteck.

In den Analysen der Bearbeitungsprozesse fällt auf, dass die Schwierigkeiten bei der Darstellung von Brüchen auf die Repräsentationsobjekte Kreis und Strecke beschränkt sind. Die Schwierigkeiten der Lernenden äußern sich dabei bei Kreisrepräsentationen in fehlenden Strategien zum Aufteilen des Ganzen und bei Strecken in fehlerhaften Bezügen zwischen Teil und Ganzem. Mit Bezug auf die repräsentierten Größen ist dabei festzustellen, dass die Einteilung eines Kreises in gleiche Teile das Ziel hat kongruente Kreissegmente mit dem gleichen Flächeninhalt herzustellen, während eine Strecke der Länge nach in gleich lange Abschnitte unterteilt wird. 
Auch für die Darstellung von Brüchen in einem Rechteck ist es erforderlich das ganze Rechteck in Teile mit gleichem Flächeninhalt zu teilen. Anders als für die Darstellung in einem Kreis können die Lernenden bei Rechtecken jedoch auf Handlungskonzepte aus der Behandlung von Flächeninhalten zurückgreifen, die in vielen Fällen über das Auslegen von rechteckigen Figuren eingeführt werden. In diesem Zusammenhang ist es sehr wahrscheinlich, dass das Unterteilen in gleiche Flächen in einem Rechteck für die Lernenden ein vertrautes Handlungskonzept ist. Die Darstellung in einer Kreisrepräsentation erfolgt dagegen über die Unterteilung des Vollwinkels in gleiche Teile. Dies ist für Brüche, deren Nenner eine Potenz von 2 ist, über die Strategie des fortgesetzten Halbierens zumeist problemlos möglich. Zudem sind die Darstellungen dieser „Alltagsbrüche“ vielen Lernenden bereits bekannt, sodass sie eine Vorstellung davon haben, wie eine Darstellung aussehen sollte. Für andere Nenner, wie zum Beispiel Vielfache von 3, 5 und 7 verfügen die Lernenden über kein Handlungskonzept zum Einteilen des Ganzen. Aus diesem Grund bleibt ihnen in vielen Fällen nichts anderes übrig, als durch Ausprobieren (zeichnerisch oder durch mentale Simulation) eine Einteilung herzustellen, die den Anforderungen der Kongruenz der Teile entspricht. Dieser Prozess ist in hohem Maße fehleranfällig.

In den Bearbeitungen zum Repräsentationstransfer auf die Repräsentation in einer Strecke ist festzustellen, dass die Lernenden häufig nicht die ganze Strecke als Ganzes betrachten, sondern die Verfahren nur auf Teile der Strecke anwenden oder falsche Bezugsgrößen nutzen. In diesen Situationen ist zu erkennen, dass häufig Strukturelemente der natürlichen Zahlen, z.B. in Form einer komponentenweisen Betrachtung von Zähler und Nenner, übertragen werden. Eine mögliche Erklärung für diese Fehler und Fehlerstrategien beim Transfer auf eine Strecke ist, dass das Vorwissen der Lernenden im Umgang mit Strecken sich vor allem auf das Messen von Längen beschränkt, wobei ausgezählt wird, aus wie vielen Längeneinheiten eine Strecke besteht. Das Konzept des Einteilens einer Strecke in gleich lange Abschnitte ist für die Lernenden vermutlich in vielen Fällen neu, sodass sie eher auf vertraute Handlungskonzepte zurückgreifen, die Strecken in einen engen Zusammenhang mit natürlichen Zahlen setzen.

Es kann angenommen werden, dass die vorhandenen Handlungskonzepte für den Umgang mit verschiedenen Darstellungen dazu führen, dass die neuen anschaulichen Handlungskonzepte des Teilens in gleiche Teile und Markierens einer bestimmten Anzahl von Teilen sowie das Vergröbern einer Einteilung als Zusammenfassen einer bestimmten Anzahl von Teilen zu einem neuen größeren Teil nicht übertragen werden. 
Transfer zwischen Sachkontexten: ̈hnlich wie beim Transfer zwischen Repräsentationsobjekten sind auch Sachkontexte mit bestimmten Handlungskonzepten verbunden. Diese Handlungskonzepte haben einen weitreichenden Einfluss auf die inhaltlichen Deutungen und Bezüge, die die Lernenden während der Bearbeitung aushandeln und herstellen. Bereits in der ersten Datenerhebung mit dem inhaltlichen Schwerpunkt der Bruchherstellung und ikonischen Darstellung von Brüchen war zu erkennen, dass selbst in Aufgaben, in denen ein möglicher Sachkontext angedeutet wurde und die eigentlichen Darstellungen und Lösungsverfahren auf einer innermathematischen Ebene dargestellt wurden, die Deutungen der Lernenden beeinflusst wurden. So reichte es aus, dass bei der Darstellung von Brüchen in einem Kreis oder einem Rechteck, die mit dem Teilen eines Kuchens oder einer Pizza verbunden wurden, gegenständliche Handlungskonzepte, wie Schneiden und vor allem Wegnehmen, die Übersetzung zwischen rechnerischen Verfahren und ikonischen Veranschaulichungen dieser Handlungen beeinträchtigte und zu fehlerhaften Schlüssen der Lernenden führten, sodass ein Transfer ausblieb, unvollständig oder fehlerhaft war.

Wesentlich deutlicher kam der Einfluss von mit einem Sachkontext verbundenen Handlungskonzepten bei der Bestimmung und Berechnung von Anteilen beliebiger Größen zum Tragen. In den Bearbeitungen der Lernenden ist zu erkennen, dass ein Transfer zwischen Sachkontexten vor allem vom Herstellen von Bezügen zwischen zwei Situationen abhängt. Kann zu einer der beiden Sachsituationen kein Handlungskonzept aktiviert werden oder ist das Handlungskonzept fehlerhaft, werden falsche Bezüge zwischen den Situationen hergestellt, was in den Bearbeitungen vor allem in der Wahl der falschen Bezugsgröße bei der Bildung von Anteilen dokumentiert ist.

Hervorzuheben sind in diesem Zusammenhang die Bearbeitungen des zweiten unvollständigen Beispiels der zweiten Datenerhebung, in der ein gemeinsamer Lohn auf zwei Jugendliche aufgeteilt werden sollte, die unterschiedlich lange gearbeitet haben. In dieser Sachsituation sind drei unterschiedliche Größen enthalten, die für eine Strukturierung der Sachsituation miteinander in Beziehung gesetzt werden müssen: Die zwei Jugendlichen, der Lohn als Geldbetrag und die Anzahlen an geleisteten Arbeitsstunden. Ohne ein hinreichendes Verständnis des Sachkontexts wurde von vielen Lernenden nicht erkannt, dass der Geldbetrag gemäß der Summe der Arbeitsstunden aufgeteilt werden musste, um gerecht auf die zwei Jugendlichen aufgeteilt werden zu können. Hierzu ist es jedoch notwendig zu wissen, dass Lohn im allgemeinen in Einheiten für eine geleistete Arbeitsstunde ausgezahlt wird. Es konnte beobachtet werden, dass dieses Konzept vielen Lernenden nicht vertraut war und so zu Schwierigkeiten bei der Strukturierung der Sachsituation, der Analogie- 
bildung zum Lösungsbeispiel und letztendlich zum Ausbleiben des Transfers führen konnte.

Ähnliche Beobachtungen konnten auch in der Transferaufgabe zur Fahrradkontrolle dokumentiert werden. Hier zeigte sich jedoch weniger der Einfluss der Handlungskonzepte des Sachkontexts als Schwierigkeit, sondern vielmehr die Mehrschrittigkeit des Lösungsweges als unvertrautes Handlungskonzept. Aus einem vorgegebenen Anteil, sollte auf einen anderen Anteil geschlossen werden. Dazu war es notwendig von dem vorgegeben Anteil zunächst auf einen Teil zu schließen, um das Ganze oder den gesuchten Anteil berechnen zu können. Diese Mehrschrittigkeit des Lösungswegs war für viele Lernende neu und führte zu Schwierigkeiten und fehlerhaften Lösungen.

Die Analysen der Bearbeitungsprozesse deuten darauf hin, dass neben den Handlungskonzepten, die mit einem Sachkontext verbunden werden, auch die Aufgabenstruktur bzw. die Struktur eines Lösungswegs ein Handlungskonzept darstellt, das sofern es für die Lernenden neu ist, zunächst erschlossen werden muss, bevor bekannte Verfahren übertragen und angewendet werden können.

Open Access Dieses Kapitel wird unter der Creative Commons Namensnennung 4.0 International Lizenz (http://creativecommons.org/licenses/by/4.0/deed.de) veröffentlicht, welche die Nutzung, Vervielfältigung, Bearbeitung, Verbreitung und Wiedergabe in jeglichem Medium und Format erlaubt, sofern Sie den/die ursprünglichen Autor(en) und die Quelle ordnungsgemäß nennen, einen Link zur Creative Commons Lizenz beifügen und angeben, ob Änderungen vorgenommen wurden.

Die in diesem Kapitel enthaltenen Bilder und sonstiges Drittmaterial unterliegen ebenfalls der genannten Creative Commons Lizenz, sofern sich aus der Abbildungslegende nichts anderes ergibt. Sofern das betreffende Material nicht unter der genannten Creative Commons Lizenz steht und die betreffende Handlung nicht nach gesetzlichen Vorschriften erlaubt ist, ist für die oben aufgeführten Weiterverwendungen des Materials die Einwilligung des jeweiligen Rechteinhabers einzuholen. 